\title{
Reflector stack optimization for Bulk Acoustic Wave resonators
}

Sumy Jose 
Reflector stack optimization for Bulk Acoustic Wave resonators

Sumy Jose 


\section{The graduation committee consists of:}

$\begin{array}{lll}\text { Chairman: } & \text { Prof. dr. ir. A. J. Mouthaan } & \text { University of Twente } \\ \text { Secretary: } & \text { Prof. dr. ir. A. J. Mouthaan } & \text { University of Twente } \\ \text { Promoter: } & \text { Prof. dr. J. Schmitz } & \text { University of Twente } \\ \text { Asst. promoter: } & \text { Dr. ir. R. J. E. Hueting } & \text { University of Twente } \\ \text { Referent/expert: } & \text { Dr. ir. A.B.M. Jansman } & \text { NXP Semiconductors } \\ \text { Members: } & \text { Prof. dr. P. Muralt } & \text { EPFL, Switzerland } \\ & \text { Dr. ir. R. J. Wiegerink } & \text { University of Twente } \\ & \text { Prof. dr. ir. G. J. M. Krijnen } & \text { University of Twente } \\ & \text { Prof. dr. K.J. Boller } & \text { University of Twente }\end{array}$

This research was supported by the Dutch Ministry of Economic Affairs in the framework of the Point one project MEMSLand and carried out at the Semiconductor Components group, MESA+ Institute of Nanotechnology, University of Twente, The Netherlands.

PhD thesis - University of Twente, Enschede, The Netherlands

Title: Reflector stack optimization for Bulk Acoustic Wave resonators

Author: Sumy Jose

ISBN: 978-90-365-3297-6

DOI: $10.3990 / 1.9789036532976$

Cover: He-Ion microscope image of the cross section of an SMR (Chapter 5)

Copyright (C) 2011 by Sumy Jose

All rights reserved. No part of this publication may be reproduced, stored in a retrieval system, or transmitted, in any form or by any means, electronic, mechanical, photocopying, recording or otherwise, in whole or in part without the prior written permission of the copyright owner. 


\title{
REFLECTOR STACK OPTIMIZATION FOR BULK ACOUSTIC WAVE RESONATORS
}

\section{DISSERTATION}

\author{
to obtain \\ the degree of doctor at the University of Twente, \\ on the authority of the rector magnificus, \\ prof. dr. H. Brinksma, \\ on account of the decision of the graduation committee, \\ to be publicly defended \\ on Tuesday the $13^{\text {th }}$ of December at 16.45
}

by

Sumy Jose

born on the $17^{\text {th }}$ of January 1982

in Cochin -18, Kerala, India 
This dissertation is approved by:

Prof. dr. Jurriaan Schmitz (promoter)

Dr. ir. Raymond J. E. Hueting (supervisor) 
To my parents 



\section{Contents}

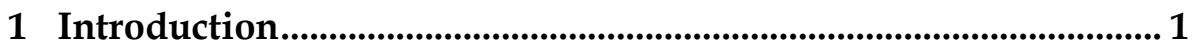

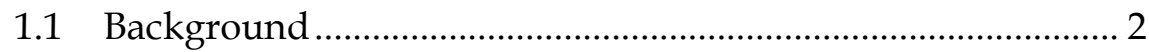

1.1.1 Filter operation principle ................................................. 4

1.2 Problem description and objective ........................................... 5

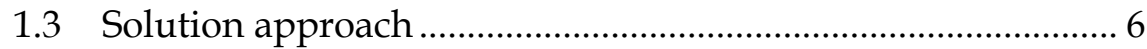

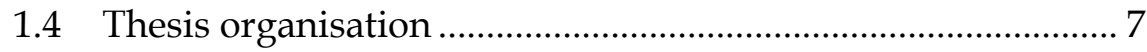

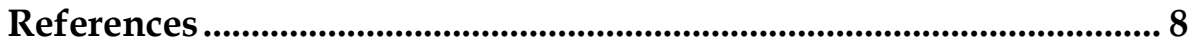

2 Bulk Acoustic Wave Devices: Basics ............................................. 10

2.1 BAW resonator concept...................................................... 11

2.1.1 BAW resonator configurations ................................. 12

2.1.2 From piezoelectricity to impedance curves ................ 14

2.2 BAW Modeling .................................................................... 16

2.2.1 The physics based 1-D Mason model.......................... 16

2.2.2 The modified Butterworth Van Dyke (mBVD) model 19

2.3 The key performance parameters for BAW resonators ........ 20

2.3.1 The effective coupling coefficient $\left(k_{\text {eff }}^{2}\right) \ldots \ldots \ldots \ldots \ldots \ldots \ldots . . . . . . . .20$

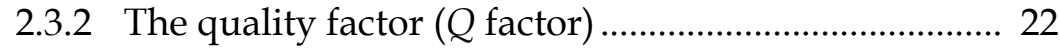

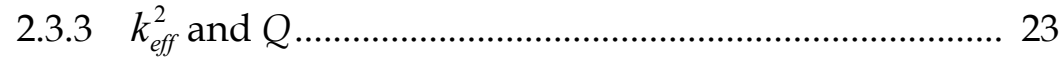

2.4 Loss mechanisms and $Q$ factor........................................... 24

2.4.1 Acoustic leakage through the reflector stack .............. 25

2.5 The acoustic dispersion ....................................................... 28

2.5.1 Introduction ............................................................ 28

2.5.2 Eigen mode concept for dispersion curves ................ 30

2.5.3 Construction of dispersion curves............................. 31

2.5.4 Types of dispersion..................................................... 33

2.6 Spurious resonances and its suppression............................. 35

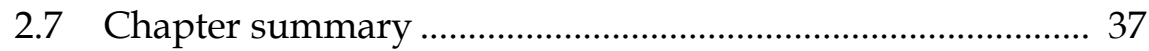


References ........................................................................................... 38

3 Reflector Stack Design ................................................................. 43

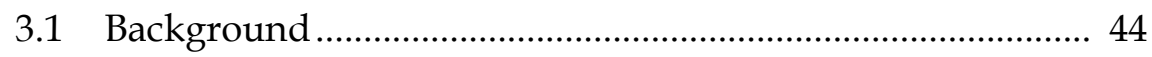

3.2 Stop-band theory based approaches ..................................... 46

3.2.1 The basic stop-band theory approach......................... 46

3.2.2 The phase error approach ......................................... 51

3.2 Diffraction grating based approaches ..................................... 56

3.3.1 The Diffraction Grating Method (DGM) ................... 56

3.3.2 An Alternative Diffraction Grating Method (ADGM) 59

3.4 2D FEM simulations ............................................................. 61

3.5 Comparison of the design approaches................................. 63

3.6 Conclusions ........................................................................... 64

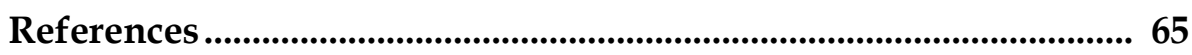

4 Acoustic dispersion of SMRs with optimized reflector stacks.. 68

4.1 Influence of reflector stack design on acoustic dispersion... 69

4.2 Flipping of the dispersion relation in SMRs.......................... 73

4.3 Flipping of the dispersion curve extended to FBARs ........... 80

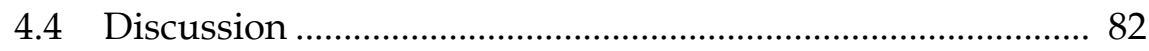

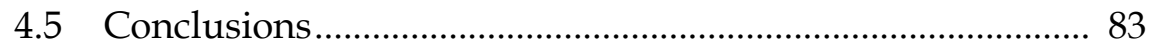

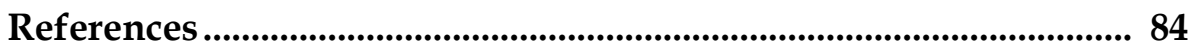

5 High $Q$ Solidly Mounted Resonators: Experimental Results .. 86

5.1 BAW reflector experiments.................................................. 87

5.2 Measurement set-up .......................................................... 88

$5.3 Q$ improvement of the dielectric $\left(\mathrm{SiO}_{2} / \mathrm{Ta}_{2} \mathrm{O}_{5}\right)$ stacks........ 90

$5.4 Q$ improvement of dielectric-metal $\left(\mathrm{SiO}_{2} / \mathrm{W}\right)$ stacks......... 94

5.4.1 $Q$ factor Analysis.......................................................... 96

5.4.2 Diffraction grating method (DGM) stack analysis... 105

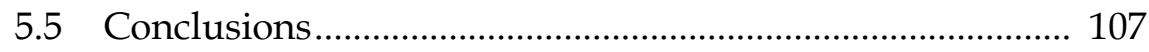

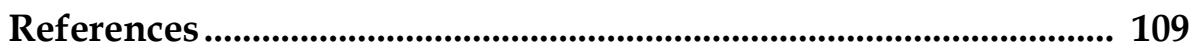

6 Conclusions and recommendations............................................. 111

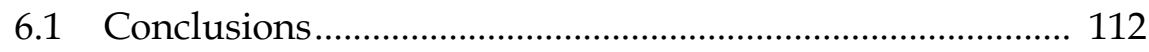

6.2 Suggestions for further research ........................................ 113

A COMSOL: Multiphysics modeling and simulation software.. 115

B FEM simulation vs. Mason model ............................................... 118 
C $Q$ extraction method................................................................. 120

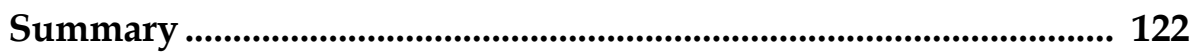

Samenvatting................................................................................................. 124

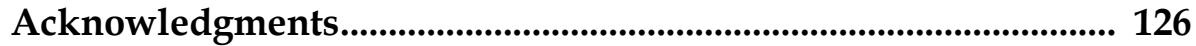

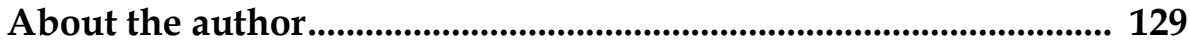





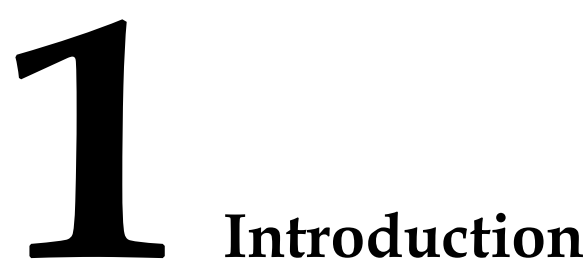

This chapter presents a general introduction to this thesis. Objectives of this research and the background related to Bulk Acoustic Wave (BAW) resonators are presented. The chapter ends with a discussion of solution approaches and an outline of the thesis. 
In his book "The art of rhetoric" the ancient Greek philosopher, Aristotle (384 BC - 322 BC) annotates speech as one of the approaches for pisteis (persuasion) [1]. Sound being a communication medium for speech was further comprehended and interpreted by him as "contractions and expansions of the air falling upon and striking the air which is next to it...", a very good expression of the nature of wave motion [2]. Ever since its development through the late 17th century, Acoustics, the science of sound, has evolved as a diversified science that deals with the study of propagation of sound in gases, liquids, and solids including vibration, audible sound, ultrasound and infrasound [3].

Material progression in Acoustics, after the discovery of piezoelectricity by the Curie brothers in 1880, led to the evolution of electro-acoustic devices in early 20 $0^{\text {th }}$ century [4]. Since then, these devices have found their use in a multitude of components such as filters, resonators, oscillators, sensors, and actuators in telecommunication, industrial and automotive applications. One among these devices is an acoustic resonator when miniaturized is termed as an acoustic microresonator. This thesis focuses on the performance optimization of a kind of microresonator, the so-called bulk acoustic wave (BAW) resonator, used for signal filtering in mobile communication systems. In this chapter we present a general introduction to the work of this thesis - application of BAW physics in performance enhancement of the devices - and the motivation and aim we were seeking for.

\subsection{Background}

BAW resonators are electro-acoustic devices that experience acoustic wave propagation and eventually vibrate at a resonance frequency related to the device dimensions. Two physical phenomena that contribute for the functioning of BAW resonators are the piezoelectric effect and mechanical (acoustical) resonance. The piezoelectric effect is an ability of a material to convert electrical energy to mechanical vibration. As will be explained in more detail in chapter 2, when an electric field is applied to a BAW resonator (see Figure 1.1.), an acoustic wave is launched in the device by piezoelectric effect. This wave resonates along the vertical direction of the device when half of the wave gets confined across the thickness of the piezoelectric layer.

The currently preferred technology for radio frequency (RF) filters is the surface acoustic wave (SAW) structure. BAW devices are receiving great interest for RF selectivity in mobile communication systems and other wireless applications as the communication bands move higher into the frequency spectrum. These devices are a consequence of advancement of MEMS (Micro-Electro-Mechanical-Systems) into RF communication and high frequency control applications [5]. Thin-film BAW devices have several advantages compared to the surface acoustic wave (SAW) resonators that had been reigning the wireless market, as they are remarkably small in size, have better power handling abilities and lower temperature coefficients leading to more stable operation [6]. From a practical point of view SAW filters have considerable drawbacks beyond 2 $\mathrm{GHz}$ whereas BAW devices up to $20 \mathrm{GHz}$ have been demonstrated [7]. A detailed review of the strengths and weaknesses for both SAW and BAW technologies is presented in [8]. Although currently it is difficult to declare the victory of one 


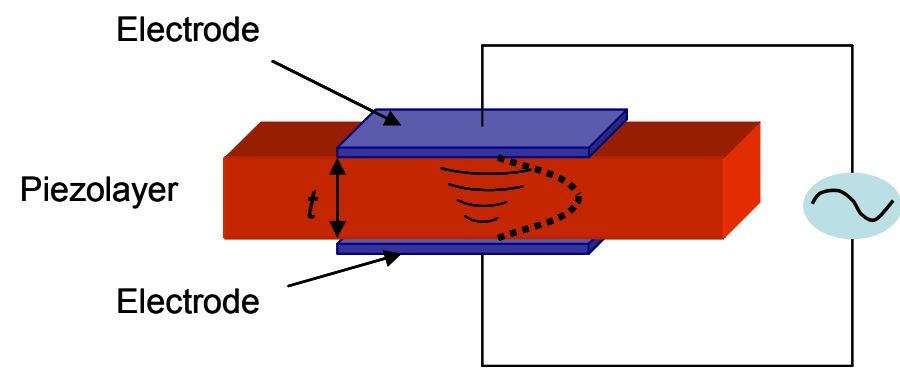

Figure 1.1 : $\quad A$ schematic of a BAW resonator. $t$ denotes the layer thickness dimension which is typically in the order of micrometers.

technology over the other [9], BAW is expected to supersede SAW as the technology of choice in many applications over the next few years as they have now evolved in performance beyond SAW and can be manufactured in a very cost competitive way using standard planar technology.

As mentioned earlier, BAW devices utilize the piezoelectric effect to generate a mechanical resonance from an electrical input. The conversion between electrical and mechanical energy is achieved using a piezoelectric material. The use of piezoelectric materials for different applications was prompted by the basic experimental and theoretical work at Bell Telephone Laboratories in the early 1960's [10]. Nevertheless, the thickness vibration mode of piezoelectric crystals was reported for an application as a transducer a decade earlier [11].

The mechanically resonant device which can be a substitute component for frequency filters in integrated electronics technology was later proposed by Newell in 1965 [12]. BAW resonators were first demonstrated in 1980 by Grudkowski et al. and Nakamura, et al. [13], [14] soon followed by Lakin and Wang [15], [16]. Preceded by the development of devices based on acoustic wave resonators by Lakin's group at TFR technologies [17], several companies [18]-[23] have been developing this technology. Currently, BAW technology is commercially available for US-PCS (Transmit band: $1.85-1.91 \mathrm{GHz}$, Receive band: $1.93-1.99 \mathrm{GHz}$ ) applications. A major limitation with the US-PCS standard is that the transmit and receive bands are close in frequency [23]. This demands BAW resonators which constitute the narrow band filters for the application to be nearly loss-free. Hence one of the important goals of BAW community is to come up with high $Q$ resonators for RF filters by minimizing the energy losses [9], [20]-[24]. 


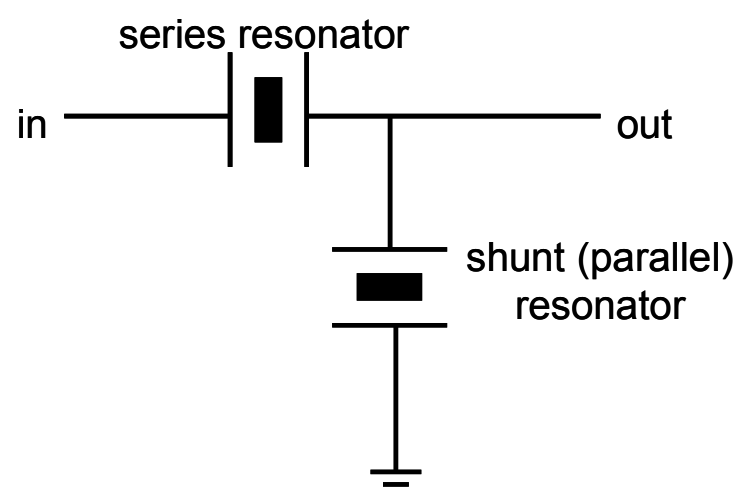

Figure 1.2 : $\quad$ Single stage section BAW ladder filter consisting of one series and one parallel resonator, the former having a higher resonance frequency by e.g. reducing the top-electrode thickness.

\subsubsection{Filter operation principle}

Thin film BAW filters which are bandpass filters are composed of BAW resonators. A bandpass filter can be implemented by electrically or mechanically (acoustically) coupling two or more resonators [6], [22], [25]. Typically two groups of resonators, series and shunt (parallel) resonators, having different resonance frequencies will be sufficient to make filters. One series resonator and one shunt resonator is called as 'stage'. Typical BAW filters consist of multiple stages. A single stage so-called BAW ladder filter consisting of one series and one parallel resonator is shown in Figure 1.2.

The working principle of a BAW filter [6], [22], [25] is illustrated in Figure 1.3. The electrical impedance of a BAW resonator has two characteristic frequencies, the resonance frequency $f_{\mathrm{R}}$ and anti-resonance frequency $f_{\mathrm{A}}$. At $f_{\mathrm{R}}$, the electrical impedance is very small whereas at $f_{\mathrm{A}}$, is very large. As mentioned above, filters are made by combining several resonators. The shunt resonator is shifted in frequency with respect to the series resonator. When the resonance frequency of the series resonator equals the anti-resonance frequency of the shunt resonator, maximum signal is transmitted from input to output of the device. At the anti-resonance frequency of the series resonator, the impedance between the input and out terminals is high and the filter transmission is blocked. And at the resonance frequency of the shunt resonator, any current flowing into the filter section is shorted to ground by the low impedance of the shunt resonator, so that the BAW filter also blocks signal transmission at this frequency. This results in the band-pass filter characteristic as shown in the figure. The frequency spacing between $f_{\mathrm{R}}$ and $f_{\mathrm{A}}$ determines the filter bandwidth. 


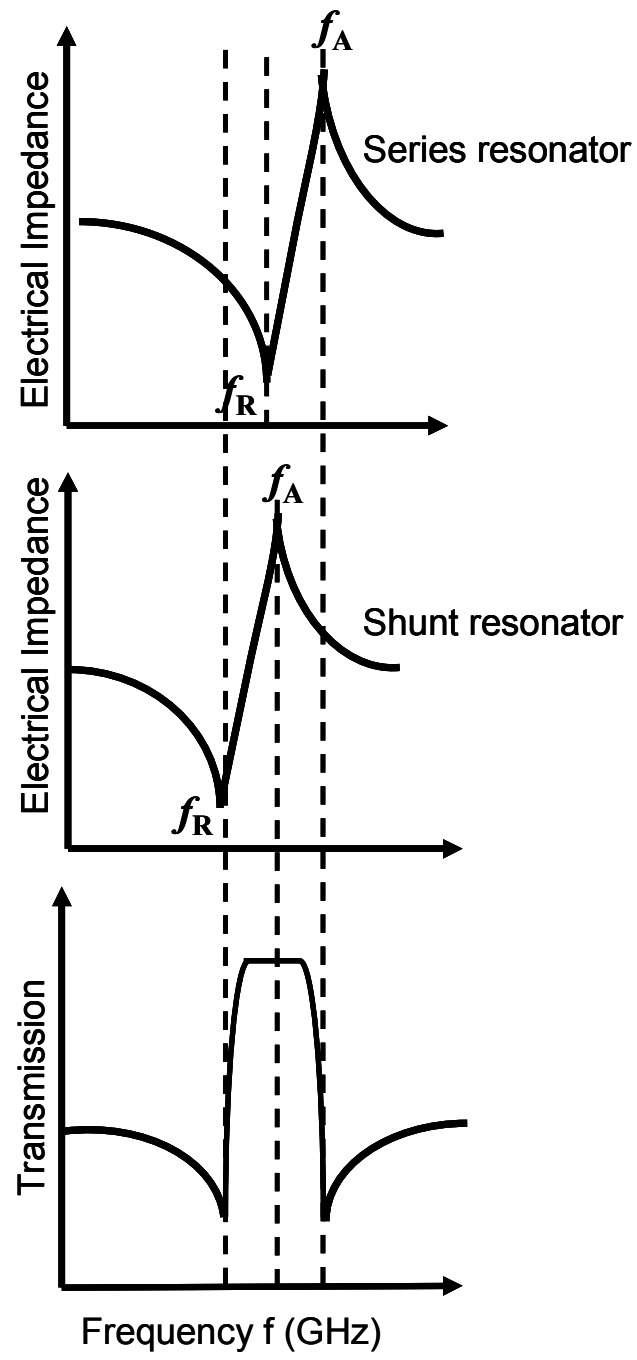

Figure 1.3:

Working Principle of a BAW filter. Top: Impedance of series resonator. Middle: Impedance of shunt resonator. Bottom: Transmission of a single stage ladder filter in terms of RF power transmission (the output of Figure 1.2) revealing the band-pass filter characteristic.

\subsection{Problem description and objective}

"A problem well stated is a problem half solved." -Charles F. Kettering (Inventor, 1876-1958)

In this thesis we are focusing on the design optimization of the basic building block of BAW filters, the BAW resonator. Essentially two types of thin-film BAW resonators have been reported, a membrane based film bulk acoustic wave resonator (FBAR) and a reflector based Solidly-Mounted BAW resonator (SMR) which is discussed in chapter 2 . Apart from the technological benefits of using SMRs discussed later in this thesis, we chose to work on SMR because this Ph.D. project was initiated in strong collaboration with NXP semiconductors, Eindhoven where only SMR technology had been explored. 
An important figure of merit, the quality factor $(Q)^{*}$ of conventional Solidly Mounted Bulk Acoustic Wave Resonators (SMRs) is traditionally limited by acoustical substrate losses [26]-[29], because the conventional quarter wave Bragg reflector employed in SMRs reflects only the longitudinal acoustic waves and not the shear waves. In order to obtain high- $Q$ SMRs, the reflector stack below the resonator should effectively reflect both the waves. Therefore, the influence of shear waves on $Q$ was reviewed earlier [26], [30]. Incidentally, the shear wave velocity being about half that of longitudinal wave velocity [29], quarter wave Bragg reflector designed for the reflection of longitudinal waves exactly correspond to the full transmission condition for shear waves.

This quandary was under investigation since 2005 [27]-[30]. Some optimized stacks which are different from quarter wave stack have been reported for specific material combinations [27]-[30] based on numerical calculations. But to our knowledge a systematic design procedure with a solid theoretical background was never reported. The main objective of this work is to come up with a systematic design procedure so as to design reflector stacks for SMRs that effectively reflect both longitudinal and shear waves. The motivation behind this objective is to devise high $Q$ resonators by minimizing these substrate losses. The thesis aims to contribute to a better understanding of the device physics aspects of BAW resonators in context of the longitudinal and shear wave co-optimization.

\subsection{Solution approach}

"Let us return from optics to mechanics and explore the analogy to its full extent. In optics, the old system of mechanics corresponds to intellectually operating with isolated mutually independent light rays. The new undulatory mechanics corresponds to the wave theory of light. "

- Erwin Schrödinger, Nobel lecture, 1933.

For solving the problem of dual wave reflection in a Bragg reflector, we dived into the field of Optics [31] where the Bragg reflectors originated. In an exhaustive literature survey, we noticed that dual wavelength Bragg reflectors for the use in optoelectronic devices had been reported [32]. This instigated us to go further into the field of thin-film optics to find a solution for our quandary. Thin-film optical filters and resonators using Bragg reflectors were well-known [33], [34]. Bragg reflectors in thin-film optics using alternate layers of high and low refractive indices are analogous to the Bragg reflectors in acoustics which uses alternating layers of high and low acoustic impedances [12], [35]. However, an important difference is that the BAW filters needed to reflect longitudinal and shear acoustic waves having different velocities at the same resonant frequency whereas in optical filters, light with a fixed velocity is filtered at different wavelengths.

The primary reasons for processing electrical signals using acoustic (i.e. mechanical waves), rather than electromagnetic (EM) waves, are that device size can be orders of magnitude smaller due to a much lower mechanical wavelength compared to the EM wavelength at a given frequency. However, in both the domains of optics and acoustics,

\footnotetext{
* The quality factor accounts for the losses associated with a resonator. This is explained in detail in chapter 2.
} 
the field equations have the same mathematical form which implies any technique used in EM field theory can be applied to acoustics with appropriate transformation analogies [36]. The work of this thesis ascertains that the principles of one physical domain (optics) can be inherited for the application in another physical domain (acoustics), the wave concepts being the same in all the domains.

\subsection{Thesis organisation}

This thesis is organized as follows.

Chapter 2 introduces the basics of BAW device physics. The background to the subject of thin-film BAW devices, the basic working principle and BAW configurations as well as the relevant models to be used are discussed here. A concise introduction to the terminologies associated with BAW resonators is also presented. This exposes the reader to the necessary theoretical background required to read ensuing chapters.

Chapter 3 is the heart of this thesis as it deals with the novel reflector stack designs to effectively reflect both longitudinal and shear waves in SMRs. The design approaches discussed here are derived from its background from optics. It has been demonstrated using FEM simulations that the design schemes are applicable for various material combinations.

Chapter 4 is a study on the acoustic dispersion of SMRs with optimized reflector stacks. This chapter presents the influence of the reflector stack design on the acoustic dispersion of SMRs. Depending on the reflector stack design approaches discussed in chapter three, the resonators exhibit different dispersion types: type I or type II. First, the basic concepts as well as some simulation studies will be presented. A rule of thumb for flipping the dispersion curve to type I, the preferred dispersion type in practice, is proposed and discussed.

Chapter 5 discusses the experiments carried out on SMRs based on various reflector stacks designed with the approaches discussed in chapter 3 . The stacks realized were of two different material combinations; one consisting of dielectrics only $\left(\mathrm{SiO}_{2} / \mathrm{Ta}_{2} \mathrm{O}_{5}\right)$ and the other of a dielectric-metal combination $\left(\mathrm{SiO}_{2} / \mathrm{W}\right)$. The electrical characterization of the resonators is presented. The improvements in the reflection of the reflector stacks will be reflected on the $Q$ factor measurements from the impedance curves. The chapter also presents the influence of increased top-oxide on reflector stack design. The results corroborate the theory presented in previous chapters. Finally conclusions are drawn based on the experimental results.

Chapter 6 summarizes the thesis, and presents some possible future work in the direction of the study presented in this thesis. 


\section{References}

[1] Aristotle, The Art of Rhetoric, translated by John Henry Freese, Harvard University Press, 1926.

[2] M. R. Cohen and I. R. Drabkin, A Source book in Greek Science, pp. 289, Harvard University Press, 1948.

[3] L. L. Beranek, Acoustics, Acoustical Society of America, 1954.

[4] A. Ballato, "Piezoelectricity - Old Effect, New Thrusts," IEEE Transactions on Ultrasonics Ferroelectrics and Frequency Control, vol. 42, no.5, pp. 916-926, 1995.

[5] R. Aigner, J. Ella, H. J. Timme, L. Elbrecht, W. Nessler, and S. Marksteiner, "Advancement of MEMS into RF-filter applications," International Electron Devices Meeting, Technical Digest, pp. 897-900, 2002.

[6] H. P. Loebl, C. Metzmacher, R.F. Milsom, P. Lok, F. Van straten and A. Tuinhout, "RF bulk acoustic resonators and filters," Kluwer Journal of Electroceramics, 12, pp.109-118, 2004.

[7] K. M. Lakin, J.R. Belsick, J.P. McDonald, K.T. McCarron, and C.W. Andrus, “Bulk acoustic wave resonators and filters for applications above $2 \mathrm{GHz}$," IEEE MTT-S Int. Symp. Digest, pp. 1487-1490, June 2002.

[8] R. Aigner, "SAW and BAW technologies for RF filter applications: A review of the relative strengths and weaknesses," Proc. IEEE Ultrasonics Symposium, pp. 582-589, 2008.

[9] R. Ruby, "Review and comparison of Bulk Acoustic Wave FBAR, SMR technology," Proc. IEEE Ultrasonics Symposium, pp. 1029-1040, 2007.

[10]F. S. Hickernell, "The piezoelectric semiconductor and acoustoelectronic device development in the sixties," IEEE Transactions on Ultrasonics Ferroelectrics and Frequency Control, vol. 52, no.5, pp. 737-745, 2005.

[11]W. G. Cady, "Piezoelectric Equations of State and Their Application to ThicknessVibration Transducers," The Journal of the Acoustical Society of America, vol. 22, pp. 579583 , September 1950.

[12]W. E. Newell, "Ultrasonics in Integrated Electronics," Proceedings of the Institute of Electrical and Electronics Engineers, vol. 53, pp. 1305-1309, 1965.

[13]T. W. Grudkowski, J. F. Black, T. M. Reeder, D. E. Cullen, and R. A. Wagner, "Fundamental-Mode Vhf-Uhf Miniature Acoustic Resonators and Filters on Silicon," Applied Physics Letters, vol. 37, pp. 993-995, 1980.

[14] K. Nakamura, H. Sasaki, and H. Shimizu, "A Piezoelectric Composite Resonator Consisting of a ZnO Film on an Anisotropically Etched Silicon Substrate," Proc. of 1st Symp. on Ultrasonic Electronics, Tokyo, 1980.

[15] K. M. Lakin and J. S. Wang, "UHF Composite Bulk Wave Resonators," IEEE Transactions on Sonics and Ultrasonics, vol. 28, pp. 394-394, 1981.

[16]K. M. Lakin and J. S. Wang, "Acoustic bulk wave composite resonators," Applied Physics Letters, vol. 38, pp. 125-127, 1981.

[17] K.M. Lakin, J.S. Wang, G.R. Kline, A.R. Landin, Y.Y. Chen, and J.D. Hunt, " Thin film resonators and filters, "Proc. IEEE Ultrasonics Symposium, pp. 466-475, 1982.

[18]R. Ruby and P. Merchant, "Micromachined Thin Film Bulk Acoustic Resonators," Proceedings of the IEEE International Frequency Control Symposium, pp. 135-138, 1994. 
[19] R. Aigner,"Volume manufacturing of BAW-filters in a CMOS fab," Proc. International Symposium on acoustic wave devices for future mobile communications systems, pp. 129134, 2004.

[20] J. W. Lobeek, R. Strijbos, A. B. M. Jansman, N. X. Li, A. B .Smolders and N. Pulsford, "High- $Q$ BAW resonator on $\mathrm{Pt} / \mathrm{Ta}_{2} \mathrm{O}_{5} / \mathrm{SiO}_{2}$-based reflector stack," Proc. IEEE Microwave Symposium, pp.2047-2050, 2007.

[21] R. Strijbos, A. B. M. Jansman, J. W. Lobeek, N. X. Li and N. Pulsford, “Design and characterization of high-Q Solidly-Mounted Bulk Acoustic Wave filters," Proc. IEEE Electronic components and technology conference, pp.169-174, 2007.

[22] F. Z. Bi and B. P. Barber, "Bulk acoustic wave RF technology," IEEE Microwave magazine, pp. 65-80, October 2008.

[23] E. Schmidhammer, B. Bader, W. Sauer, M. Schmiedgen, H. Heinze, C. Eggs, and T. Metzger, "Design flow and methodology on the design of BAW components," IEEE MTT-S Int. Symp. Digest, pp. 233-236, June 2005.

[24] K. M .Lakin, G. R. Kline and K. T. McCarron, "High- $Q$ microwave acoustic resonators and filters," IEEE Trans. Microwave Theory and Techniques, 41(12), pp. 2139-2146, 1993.

[25] R. Aigner, "MEMS in RF filter applications: Thin-film bulk acoustic wave technology," Wiley Interscience: Sensors Update, vol. 12, pp. 175-210, 2003.

[26] J. Kaitila, "Review of wave propagation in BAW thin film devices progress and prospects," Proc. IEEE Ultrasonics Symposium, pp. 120-129, 2007.

[27] S. Marksteiner, J. Kaitila, G. G. Fattinger and R. Aigner, “Optimization of acoustic mirrors for Solidly Mounted BAW resonators," Proc. IEEE Ultrasonics Symposium, pp. 329-332, 2005.

[28] S. Marksteiner, G. G. Fattinger, R. Aigner and J. Kaitila, Acoustic Reflector for a BAW resonator providing specified reflection of both shear wave and longitudinal waves, US patent: 006933807B2., Aug. 2005.

[29]G. G. Fattinger, S. Marksteiner, J. Kaitila, and R. Aigner, "Optimization of acoustic dispersion for high performance thin film BAW resonators," Proc. IEEE Ultrasonics Symposium, pp. 1175-1178, 2005.

[30]G. G. Fattinger, "BAW resonators design considerations -An overview," Proc. IEEE International Frequency Control Symposium, pp. 762-767, 2008.

[31] Optics, Eugen Hecht, 3rd edition, Addison Wesley Longman Inc., 1998.

[32]C.P. Lee, C. M. Tsai, J. S. Tsang, “Dual-wavelength Bragg reflectors using GaAs/AlAs multilayers, " Elect. Lett., vol. 29, no.22, pp. 1980-1981, 1993.

[33] Thin film Optical Filters, H. A. Macleod, Adam Hilger, 1986.

[34] Wavelength Filter in Fiber Optics, H. Venghaus, Springer, 2006.

[35] B. A. Auld, C. F. Quate, H. J. Shaw and D. K. Winslow, "Acoustic quarter-wave plate at microwave frequencies," Applied Physics Letters, vol. 9, no.12, pp. 436-438, 1966.

[36] B. A. Auld, "Application of Microwave Concepts to the Theory of Acoustic Fields and Waves in Solids, "IEEE Transactions on Microwave Theory and Techniques, vol. 17, no.11, pp. 2844-2849, 2010. 



\section{Bulk Acoustic Wave Devices: Basics}

This chapter introduces the basics of Bulk Acoustic Wave (BAW) device physics that will serve as a background for ensuing chapters. A literature study on relevant models for $B A W$ resonators is presented and the main resonator parameters are explained. A concise introduction to the terminologies associated with $B A W$ resonators is also presented. 
This chapter presents the basic physical concepts of Bulk Acoustic Wave (BAW) devices. The BAW resonator concept and the two generally adopted configurations are introduced in section 2.1. The existing models for BAW device operation are reviewed in section 2.2. Section 2.3 discusses the key performance parameters for BAW resonators, section 2.4 deals with the loss mechanisms in thin film BAW resonators and its association with the quality factor and section 2.5 treats the acoustic dispersion relation and the types of dispersion. Spurious mode and its suppression are discussed in section 2.6. Section 2.7 summarizes the chapter.

\subsection{BAW resonator concept}

BAW resonators exploit the piezoelectric effect [1] of a thin piezoelectric film for obtaining resonance [2], [3]. The simplest configuration of a BAW resonator is a thin piezoelectric film sandwiched between two metal electrodes as shown in Figure 2.1. When a dc electric field is created between the electrodes, the structure is mechanically deformed by the inverse (or converse) piezoelectric effect [4]. When applying an ac electric field, the electric signal is transformed into a mechanical or acoustic wave in the device. This longitudinal acoustic wave launched into the device propagates along the electric field and is reflected at the electrode/air interfaces. As the name suggests a longitudinal wave is a wave in which the particle displacement is in the same $(z)$ direction as that of the wave propagation. The thin film BAW resonators make use of this so-called thickness extensional (TE) vibration mode of a piezoelectric film [5], [6].

At the fundamental resonance, half the wavelength of the longitudinal acoustic wave is equal to the total thickness of the piezoelectric film. The resonance (or series resonance) frequency $f_{\mathrm{R}}$ is determined approximately by the thickness $t$ of the piezoelectric film [2], [3]:

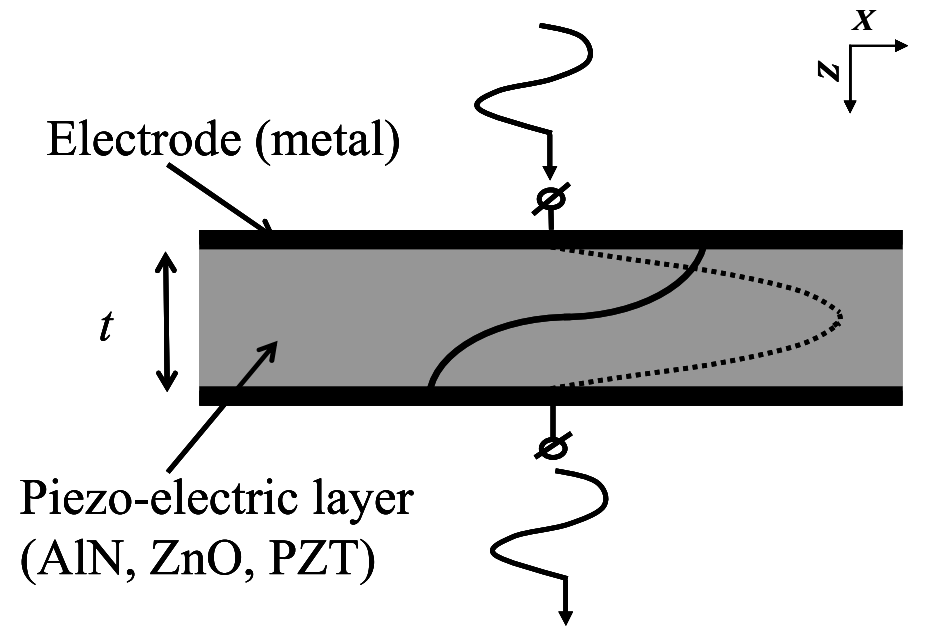

Figure 2.1: A schematic cross-section of a free standing (stress is zero at the electrode/air interfaces) $B A W$ resonator with infinite lateral dimensions. The dashed line (stress) and the solid line (displacement) indicate half wavelength of the acoustic wave vertically trapped in the piezoelectric layer indicating fundamental thickness resonance (the TE mode, see main text). The wavelength of the applied electric signal is not to the scale. 


$$
f_{\mathrm{R}}=\frac{v}{\lambda} \approx \frac{v_{\mathrm{L}}}{2 t}
$$

where $v_{\mathrm{L}}$ is the longitudinal acoustic velocity in the normal direction in the piezoelectric layer, $t$ is the thickness of the piezoelectric film, and $\lambda$ is the acoustic wavelength of longitudinal wave. In practice, the frequency $f_{R}$ is different from eq. (2.1), since the acoustic properties of all other layers affect the resonator performance e.g. by the massloading effect of the resonator's electrodes [2], [7]. Although eq. (2.1) is only a crude approximation it is important to note that as the sound velocity is typically in the range between $3000-11000 \mathrm{~m} / \mathrm{s}$ for most of the materials, for the desired frequency range $(1-$ $3 \mathrm{GHz}$ ), the thickness of the piezo layer is in the order of micrometers which makes the devices relatively smaller than electromagnetic structures [2],[8].

For the device to be practical, there are two widely adopted configurations. These are discussed in section 2.1.1.

\subsubsection{BAW resonator configurations}

As discussed above, the construction of a BAW resonator is rather straight-forward. It consists of a piezoelectric layer and two electrodes. The resonator must be attached somewhere. This attachment might disturb the free motion of the materials. Therefore, in practice, these resonators require an acoustic isolation from the substrate to prevent energy leakage thereby confining the acoustic wave in the resonator yielding a high quality factor (section 2.3.2).

There are two types of BAW resonator configurations, employing two different kinds of acoustic isolation from the substrate, namely the film bulk acoustic resonator (FBAR) and the solidly mounted resonator (SMR). The FBAR uses an air-gap cavity for the acoustic isolation from the substrate whereas in the case of an SMR, a reflector stack (or acoustic mirror) provides the isolation [9].

Figure 2.2 (a) shows one possible approach for an FBAR in which substantial acoustic isolation from the substrate is achieved by micro-machining an air-gap below the structure. The resonator is anchored from the sides only. As the acoustic impedance ${ }^{*}$ of air is a factor of $10^{5}$ lower than in typical solid materials, less energy is radiated into the air at the top and bottom surfaces of the electrodes [6]. In FBARs, the sandwich structure is almost mechanically floating. These membrane type BAW resonators are also called Free-standing Bulk Acoustic Resonator [10].

Figure 2.2 (b) shows a more mechanically rugged structure that is formed by isolating the resonator from the substrate with a Bragg reflector stack that is composed of alternating layers of low and high acoustic impedances located below the bottom electrode [2], [9]. The reflector stack layers are nominally quarter wavelength $(\lambda / 4)$ thick

\footnotetext{
* The acoustic impedance is a property of the medium which is the product of its mass density and the acoustic velocity of the wave in the medium [4], [12].
} 


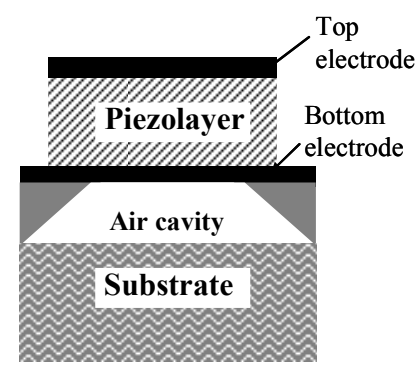

(a) FBAR

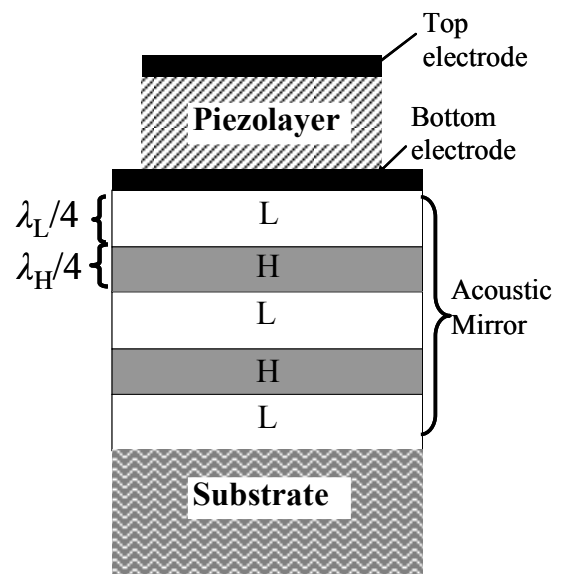

(b) SMR

Figure 2.2: $\quad$ Schematic cross-section of bulk acoustic wave resonator configurations: (a) Film Bulk Acoustic Resonator (FBAR) (b) Solidly Mounted Resonator (SMR). L and H indicate layers having a low and high acoustic impedance, respectively.

[9], [11]. The number of layers depends on the reflection coefficient required and the characteristic impedance ratio between the successive layers [9].

Good comparisons between two technologies are presented in [6], [10], [11]. The appeal of FBARs lies in the small number of layers to be manufactured and in the potentially high quality factor ( $Q$ factor) that can be achieved. On the negative side, the layer stress can cause serious problems like buckling of the structure. Membranes are very delicate to handle as soon as they are released and they are prone to damage during dicing and assembly. In addition to efficiently isolating the acoustic waves from the substrate, the membranes also prevent efficient heat transfer down to the substrate which is important for power handling. A large portion of the generated heat will not be removed by convection in air and has to travel along the lateral direction until it finds a proper heat sink. Concerning the power handling capabilities, FBAR has some principal drawbacks as well. In FBARs, the designer has to deal with harmonic resonances (overtones) of considerably high $Q$-values because the isolation to the substrate is perfect at all frequencies [6].

The realization of SMRs requires several additional layers to be deposited, which increases processing costs; however is CMOS compatible [3], [6]. At low frequencies (below $500 \mathrm{MHz}$ ) the mirror approach becomes impractical because the $\lambda / 4$ layers need to be very thick. In terms of robustness, the SMR is superior to an FBAR. There is no risk of mechanical damage in any of the standard procedures needed in dicing and assembly and there are also less problems with layer stresses in the piezolayer or the electrode layers. For BAWs requiring good power handling capabilities it is very beneficial that a direct vertical heat path through the mirror exists which reduces thermal resistance to the ambient significantly. In SMRs, harmonic overtones are highly damped because the mirror can have bad reflection at these frequencies [6]. The SMR has a lower temperature coefficient of frequency (TCF) than the FBAR, since the $\mathrm{SiO}_{2}$ layers in the reflector stack have a positive TCF, which compensates for the negative TCF of the other layers in the stack [11]. 
Another difference between the FBAR and the SMR is that the $Q$ factor of the FBAR is more dependent on the process (membrane edge-supporting configuration). Moreover, the FBAR resonator is straightforward to design without much need of two-dimensional (2-D) modeling. The $Q$ factor of the SMR is dependent on both the process and the design. Although the design of an SMR structure involves more complicated 2-D acoustic analysis, this also gives more degrees of freedom to optimize the resonator performance. The SMR provides a lower $Q$ factor compared to an FBAR due to the presence of additional reflector layers in which an acoustic wave may attenuate and escape [11].

\subsubsection{From piezoelectricity to impedance curves}

Piezoelectric materials can convert electrical energy into mechanical (or acoustical) energy and vice versa. BAW devices utilize the converse piezoelectric effect to generate a mechanical resonance from an electrical input. Conversely, the mechanical resonance is converted into electrical domain for output [12], [13].

As the piezoelectric effect is responsible for the resonance in BAW resonators, the material properties of the deposited piezoelectric film influence the performance of the resonators to some extent [2]. The most popular piezoelectric materials used in BAW devices are aluminium nitride $(\mathrm{AlN})$, zinc oxide $(\mathrm{ZnO})$ and lead zirconium titanate (PZT). Reviews of the performance of these materials for BAW applications are reported in [6],[14]. Despite of the fact that $\mathrm{ZnO}$ has in theory a slightly higher coupling coefficient than AlN it has so far not been demonstrated as a viable alternative to AIN as $\mathrm{ZnO}$ is chemically not very stable and prone to contamination in CMOS environment [6],[15]. The other prominent piezomaterial PZT is an interesting candidate with very high coupling along with extremely high dielectric constant. However, in the $\mathrm{GHz}$ range PZT appears to have too high intrinsic losses. Moreover the high dielectric constant and low acoustic velocity would result in extremely small resonators which in turn would make it very hard to control acoustic behavior [15].

For BAW devices, AIN has now been established as the piezoelectric material that offers the best compromise between performance and manufacturability [6], [11]. The use of AlN as the piezoelectric in thin film in FBAR devices was first realised by Lakin et al. in the early 1980's [16],[17]. The relatively high stiffness of AlN, high acoustic velocity, low temperature coefficient of frequency (TCF) and more importantly the compatibility with CMOS fabrication process make this material the piezoelectric of choice [10],[13]. Currently, all commercially available FBAR and SMR devices use AIN as the piezoelectric material [10].

The electrical performance of a BAW resonator is analyzed by the so-called impedance characteristics of the resonator as shown in Figure 2.3 [2], [13]. The electrical impedance of a BAW resonator is characterized by two resonances: one at the resonance (or series resonance) frequency $f_{R}$ where the magnitude of the impedance tends to its minimum value and the other one at anti-resonance (or parallel resonance) frequency $f_{\mathrm{A}}$ where the magnitude of the impedance ideally becomes infinite. 


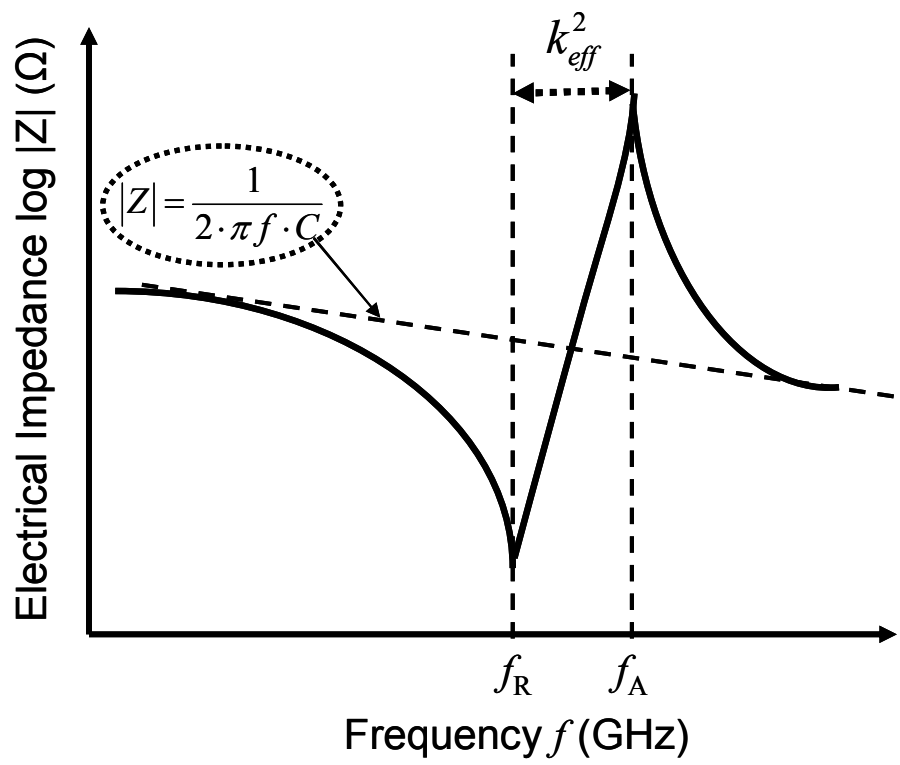

Figure 2.3: $\quad$ Impedance characteristics of a $B A W$ resonator. $f_{R}$ and $f_{A}$ represent the resonance and antiresonance frequencies respectively. $k_{\text {eff }}^{2}$, the frequency separation between the resonances $f_{R}$ and $f_{A}$ is a measure of the strength of the piezoelectric effect in the device. For frequencies other than resonance or anti-resonance, the $B A W$ resonator behaves like a Metal-InsulatorMetal (MIM) capacitor.

When an electric field is applied to the piezoelectric film sandwiched between the electrodes, the atoms and consequently the centre of dipole charges in the film are displaced [2],[4]. The crystal deforms, and the charge is attracted to the electrodes which causes an increase in current. At resonance, when the driving frequency matches the mechanical resonance frequency of the BAW resonator, the particle displacement is very large, a huge amount of charge is attracted to the electrode, and hence the impedance (ratio of voltage to current) is minimal. At anti-resonance, particle displacement is limited, though limited charge is attracted to the electrode it gets exactly compensated by the dielectric charge in the piezoelectric material. Therefore, the total charge attracted to the electrodes is negligible and hence the electrical impedance becomes enormously high.

For the frequencies other than resonance and anti-resonance, the BAW resonator behaves like a Metal-Insulator-Metal (MIM) capacitor. Therefore, far below and far above these resonances, the magnitude of the electrical impedance is proportional to $1 / f$ with $f$ as the frequency. The frequency separation between $f_{\mathrm{R}}$ and $f_{\mathrm{A}}$, is a measure of the strength of the piezoelectric effect in the device, the so-called effective coupling coefficient often represented by $k_{\text {eff }}^{2}$ (section 2.3.1). The upper limit values of the relative bandwidth $\left(\left(f_{\mathrm{A}}-f_{\mathrm{R}}\right) / f_{\mathrm{A}}\right)$ are mainly determined by the piezoelectric material, electrode material and the conditions of the surface on which the piezoelectric layer is deposited [11].

The ratio of the impedance maximum to impedance minimum is approximately equal to the $Q$ factor as long as series resistance of the leads and parasitic shunt conductance are negligible. In general, a good BAW resonator behaves like an almost ideal capacitor 


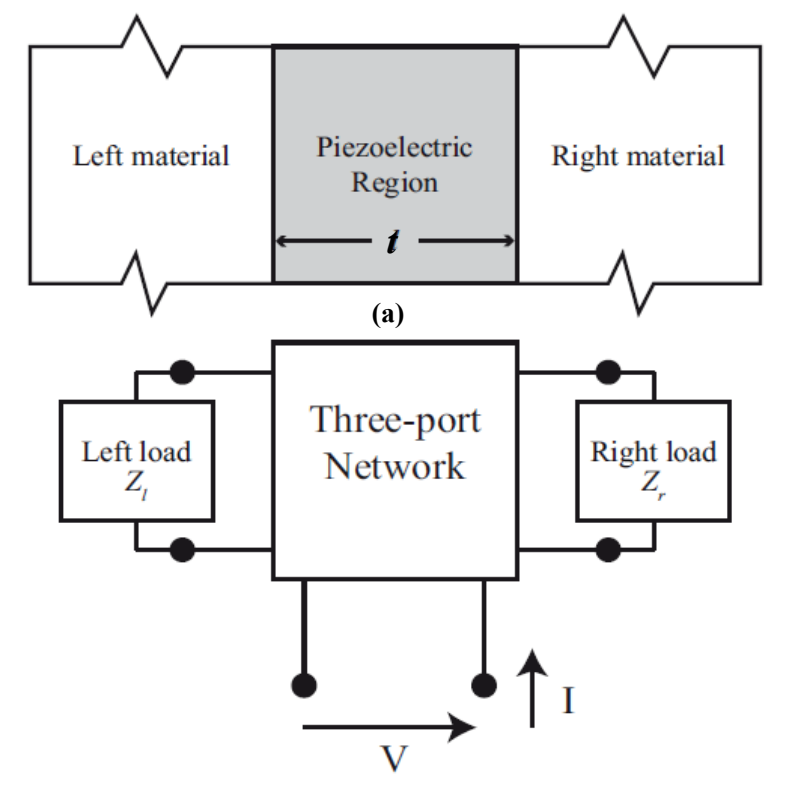

(b)

Figure 2.4: Schematic of the one-dimensional three-port Mason model: (a) Material configuration of piezoelectric material and external load materials and (b) Circuit black diagram representation showing a three-port network for piezoelectric plate. The materials on both sides of the piezoelectric plate are represented by mechanical loads $Z_{l}$ and $Z_{r}[21]$.

below $f_{\mathrm{R}}$ and above $f_{\mathrm{A}}$ and like an almost ideal inductor with varying inductance between $f_{\mathrm{R}}$ and $f_{\mathrm{A}}[6]$. The key resonator parameters, the coupling coefficient and the $Q$ factor are discussed in detail in section 2.3.

\subsection{BAW Modeling}

Time-saving modeling techniques are important tools when designing BAW resonators. Since a resonator may consist of many different layers, with different material properties, the description of such a multilayered structure requires the use of theoretical models by which the BAW physics can be modelled efficiently. Two popular models used for BAW design are the physics based one dimensional (1-D) Mason model [18] and the equivalent circuit based modified Butterworth Van Dyke (mBVD) model [12], [20],[21]. The Mason model uses an analytical approach to calculate the frequency response of the device based on the material parameters of the constituting materials, such as mass density, elastic constants, piezoelectric and dielectric constants. The mBVD model is the lumped-element electrical equivalent circuit model useful for extracting parasitic parameters [11]. Below is a summary of these two models used within the scope of this thesis.

\subsubsection{The physics based 1-D Mason model}

The Mason model is one of the most frequently used in the BAW resonator modeling [18],[21]. The model uses a transmission line concept in which the piezoelectric layer is a three port network having two acoustic ports and one electric port, as illustrated in Figure 2.4. By applying the boundary conditions at the acoustic ports, the electrical 


\begin{tabular}{|c|c|c|}
\hline Symbol & Electrical transmission line & Acoustic transmission line \\
\hline$Z_{0}$ & $\begin{array}{c}\text { Inductance per unit } \\
\text { length/ Capacitance per } \\
\text { unit length }\end{array}$ & $\begin{array}{c}\text { Characteristic acoustic } \\
\text { impedance (Mass density } \\
\text { Wave velocity) }\end{array}$ \\
\hline$\phi$ & $\begin{array}{l}\text { Phase difference of the } \\
\text { electrical wave }\end{array}$ & $\begin{array}{c}\text { Phase difference of the } \\
\text { acoustic wave }\end{array}$ \\
\hline$V(z)$ & Voltage at position $z$ & Stress at position $z$ \\
\hline$I(z)$ & Current at position $z$ & Current at position $z$ \\
\hline$Z_{\mathrm{L}}$ & Electrical impedance & Acoustic impedance \\
\hline
\end{tabular}

Table 2.1: The analogy between electrical and acoustic transmission line.

impedance at the electrical port can be calculated as a function of the frequency [8]. The analogy between electrical and acoustic transmission line is highlighted in Table 2.1.

If we consider the case of an SMR, the mechanical load on the left side $\mathrm{z}_{l}$ represents the top electrode terminated by a mechanical short. Therefore, for the boundary conditions at the top electrode holds that the stress and hence the derivative of the vertical displacement is zero. On the right hand side, $\mathrm{z}_{r}$ represents the effective mechanical impedance provided by the bottom electrode and the reflector stack, terminated by the characteristic impedance of the substrate. The impedance at the electrical port can be then given by [18], [22]:

$$
Z=\frac{1}{Y}=\frac{1}{j \omega C} \cdot\left(1-k^{2} \cdot \frac{\tan \phi}{\phi} \cdot F\left(z_{l}, z_{r}, \phi\right)\right) .
$$

$F\left(z_{l}, z_{r}, \phi\right)$ is given by

$$
F\left(z_{l}, z_{r}, \phi\right)=\frac{\left(\left(z_{r}+z_{l}\right) \cos ^{2} \phi\right)+j \sin 2 \phi}{\left.\left.\left(z_{r}+z_{l}\right) \cos 2 \phi\right)+j\left(z_{r} z_{l}+1\right) \sin 2 \phi\right)},
$$

where $\phi=\pi t / \lambda$ is half the phase across the piezoelectric plate of thickness $t, z_{l}$ and $z_{r}$ are normalized (to the acoustic impedance of the piezoelectric layer) acoustic impedances at the boundaries, and $C$ is the physical capacitance described by $\varepsilon A / t$ with $A$ the active device area. $k^{2}$ is the piezoelectric coupling coefficient given by:

$k^{2}=\frac{e^{2} / c^{D} \varepsilon^{S}}{1+e^{2} / c^{D} \varepsilon^{S}}$

where $e, c^{D}$ and $\varepsilon^{S}$ are the piezoelectric constant, elastic constant measured at constant electric displacement (superscript $D$ ) and dielectric constant measured at constant strain (superscript $S$ ) respectively. 
In the case of a simple acoustic resonator having only the piezoelectric and ideal electrodes without mass loading $\left(z_{l}=z_{r}=0\right)$, eq. (2.2) reduces to

$$
Z=\frac{1}{j \omega C} \cdot\left(1-k^{2} \cdot \frac{\tan \phi}{\phi}\right) .
$$

Eq.(2.5) gives the impedance vs. frequency characteristics of an FBAR having infinitely thin electrodes.

All structures attached to the piezoelectric plate including the mechanical effect of the electrodes, must be described in terms of equivalent terminating acoustic impedance (mechanical loads) as illustrated in Figure 2.4(b). The equivalent terminating acoustic impedance can be found by the successive use of the transmission line equation [21], [23]:

$$
Z_{\text {in }}=Z_{s} \cdot\left[\frac{Z_{l} \cdot \cos \theta+j \cdot Z_{s} \cdot \sin \theta}{Z_{s} \cdot \cos \theta+j \cdot Z_{l} \cdot \sin \theta}\right] \text {, }
$$

where $Z_{\text {in }}$ is the input acoustic impedance of the examined section in the transmission line, $Z_{1}$ the load impedance or equivalent terminating impedance attached to the section, $Z_{\mathrm{s}}$ the characteristic impedance of the section, and $\theta=2 \pi d / \lambda$ the total phase across the section where $d$ is the thickness of each layer.

The analysis of the reflector stack is most conveniently done using the fundamental equation of wave propagation. The mirror reflection $R$ is given by [24]:

$$
R=\frac{Z_{\mathrm{RS}}-Z_{\mathrm{p}}}{Z_{\mathrm{RS}}+Z_{\mathrm{p}}},
$$

where $Z_{\mathrm{p}}$ is the acoustic impedance of the piezolayer and $Z_{\mathrm{RS}}$ is the effective acoustic impedance of the layer stack below the piezolayer, including the bottom electrode, mirror layers and the substrate. Both $R$ and $Z_{R S}$ are generally complex numbers.

The Mason model together with the transmission line equation allows for calculating the transmission characteristics for longitudinal and shear waves, by just choosing the appropriate material parameters (acoustic impedance and wave velocity). Marksteiner et al. [24] found that the shear reflection characteristics of the Bragg reflector can have profound effects on the $Q$-value of a longitudinal mode resonator at antiresonance. They also suggested inspecting a logarithmic transmission of the form:

$T=10 \cdot \log _{10}\left(1-|R|^{2}\right)$,

instead of the reflection given by eq. (2.7) to resolve small differences important for high- $Q$ resonators. This practice is adopted in this thesis in the subsequent chapters. 


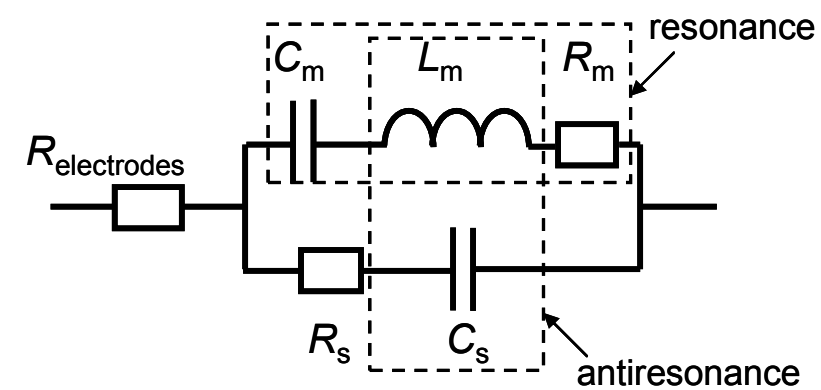

Figure 2.5: $\quad$ Modified Butterworth Van Dyke (mBVD) model with the motional $\operatorname{arm}\left(L_{m} C_{m} R_{m}\right)$ and static arm $\left(R_{s} C_{s}\right)$.

From a plot of the electrical impedance $Z$ over frequency, all relevant resonator parameters can be extracted if the material parameters and layer thickness for all the layers are known. The Mason model is suitable for optimizing both FBARs and SMRs. In general, this model will give reliable impedance curves if the material parameters are accurate. It is however, by definition not suitable for modeling spurious modes and other lateral acoustic effects and will also not predict $Q$-values of resonators accurately [6].

\subsubsection{The modified Butterworth Van Dyke (mBVD) model}

Although the physical model described above gives useful physical insight of the device, a more compact model, based on lumped parameters, is desirable for circuit designers. Apart from the physical model, there exists a compact model which is a lumped-element electrical equivalent circuit model known as the Butterworth Van Dyke (BVD) model [12], [19]. The model was further modified [20] by the addition of a parallel resistor to incorporate the parasitic components.

The modified Butterworth Van Dyke (mBVD) model is illustrated in Figure 2.5. The resonator is represented by a static arm and a motional arm. $L_{\mathrm{m}} C_{\mathrm{m}} R_{\mathrm{m}}$ - the motional arm - represents the electro-acoustic properties of the piezoelectric layer by the motional inductance $L_{\mathrm{m}}$, motional capacitance $C_{\mathrm{m}}$, and motional resistance $R_{\mathrm{m}} . R_{\mathrm{m}}$ represents the acoustic attenuation in the device. In the static arm, $C_{s}$ is the physical capacitance $\left(C_{s}=C\right.$ in eq.(2.2) ) formed by the piezoelectric layer between the electrodes. $R_{s}$ describe the dielectric losses in the material. Relectrodes represents the electrical resistance of the electrodes and the contact resistance in the measurement.

With these circuit parameters, the resonance and anti-resonance frequencies are respectively given by [12]:

$f_{\mathrm{R}}=\frac{1}{2 \pi \sqrt{L_{\mathrm{m}} C_{\mathrm{m}}}}$,

and 
$f_{\mathrm{A}}=\frac{1}{2 \pi} \sqrt{\frac{1}{L_{\mathrm{m}} C_{\mathrm{m}}}\left(1+\frac{C_{\mathrm{m}}}{C_{\mathrm{s}}}\right)}$.

Hence, the motional arm mainly determines the resonance frequency, while the antiresonance is determined by the combination of the static and motional arm.

From the mBVD circuit, the quality factor ( $Q$ factor) at $f_{\mathrm{R}}$ and $f_{\mathrm{A}}$ can be evaluated as [12], [25]:

$Q_{\mathrm{R}}^{\mathrm{mBVD}}=\frac{\omega_{\mathrm{R}} L_{\mathrm{m}}}{R_{\text {electrodes }}+R_{\mathrm{m}}}$,

where $\omega_{\mathrm{R}}=2 \pi f_{\mathrm{R}}$, and

$Q_{\mathrm{A}}^{\mathrm{mBVD}}=\frac{\omega_{\mathrm{A}} L_{\mathrm{m}}}{R_{\mathrm{S}}+R_{\mathrm{m}}}$,

where $\omega_{\mathrm{A}}=2 \pi f_{\mathrm{A}}$. The mBVD model is particularly suited for the evaluation of the resonator performance, and extraction of device properties from electrical measurements. The model only gives accurate results close to resonances [25]. This model is very practical approach for designing filters as well and the results will be as close to reality as using other commonly used model. Any circuit simulator will be able to handle the mBVD model properly. The mBVD model can be extended in many ways to include size effects, temperature effects, spurious resonances, and so on [6].

\subsection{The key performance parameters for BAW resonators}

The performance parameters to be considered for a BAW resonator design is reviewed in [10],[11],[15],[27],[28]. Although some of these reports investigate a few different parameters (such as temperature coefficient, power handling capabilities), the coupling coefficient and the quality factor determine the important characteristics of the resonator. A brief discussion about these parameters is presented in the subsections below.

\subsubsection{The effective coupling coefficient $\left(k_{e f f}^{2}\right)$}

The effective electromechanical coupling coefficient $k_{\text {eff }}^{2}$ is an important parameter for the design of BAW components. It is a measure of how efficiently the resonator converts electrical energy to mechanical energy, and vice versa [28]. The fundamental meaning of the electromechanical coupling coefficient for a "piezoelectric body" is defined by Berlincourt [29], [30] : 
$k_{e f f}^{2}=\frac{E_{\mathrm{m}}^{2}}{E_{\mathrm{a}} \cdot E_{\mathrm{e}}}$,

where $E_{\mathrm{m}}$ is the so-called mutual energy (coupled or electromechanical energy), $E_{\mathrm{a}}$ is the acoustic energy, and $E_{\mathrm{e}}$ is the electric energy.

It is to be noted that the electromechanical coupling coefficient defined for a piezoelectric material (eq. (2.4)) is a material property. Therefore $k^{2}$ is defined for a piezoelectric film, for e.g., AlN it is usually $6.6 \%$, depending on the deposition conditions [15]. The $k^{2}$ for AlN allows for filter bandwidths $>4 \%$ which is just convenient to serve narrowband communication standards [6].

For piezoelectric thin-film resonators with electrode layers and reflector stack layers, in practice an effective coupling coefficient $k_{\text {eff }}^{2}$ is defined in terms of relative spacing of the resonance frequency $f_{\mathrm{R}}$ and anti-resonance frequency $f_{\mathrm{A}}$ [2], [6], [31]:

$k_{e f f}^{2}=\frac{\pi^{2}}{4}\left(\frac{f_{\mathrm{A}}-f_{\mathrm{R}}}{f_{\mathrm{A}}}\right)=\frac{\pi^{2}}{4} \mathrm{BW}$.

The relative spacing of the resonance frequencies also determines the bandwidth of the filter.

The value of $k_{\text {eff }}^{2}$ is a measure of the strength of coupling between the acoustic and electric fields in the resonator structure as a whole. For an FBAR with ideal (infinitesimally thin, perfectly conducting) electrodes, the fractional separation of $f_{\mathrm{R}}$ and $f_{\mathrm{A}}$ is equal to $\left(4 / \pi^{2}\right)$. $k^{2}$ and thus $k_{e f f}^{2}$ is equal to the piezoelectric coupling coefficient $k^{2}$ of the piezomaterial used. For practical resonators, $k_{\text {eff }}^{2}$ depends on the electrode and the reflector stack layer configurations. Therefore, in practice, $k_{\text {eff }}^{2}$ will differ from $k^{2}$. In some circumstances, $k_{\text {eff }}^{2}$ be even larger than $k^{2}$ of the piezoelectric material used, e.g. when the acoustic impedance of the electrodes is higher than that of the piezoelectric film [10],[15],[32]. This is due to an improved match between the acoustic standing wave and the linear electric field in the piezoelectric.

Although there are various definitions in use by different groups, the definition by eq. (2.14) has been claimed as "optimist's favorite" [15]. The factors directly influencing $k_{\text {eff }}^{2}$ are associated with electro-acoustic energy conversion. The $k_{\text {eff }}^{2}$ is a maximum for the maximum overlap of electric and acoustic fields. The spacing of resonance and antiresonance will be modified when taking the additional support layers such as reflector stack layers into consideration. In most cases the additional layers will reduce the relative spacing. The AIN based FBAR gives an improved $k_{\text {eff }}^{2}(6.9 \%$ versus $6.5 \%$ at $2 \mathrm{GHz}$ ) compared to the SMR due to the existence of some stored energy outside the piezoelectric, in the reflector stack layers [11]. However, the coupling coefficient in SMRs also can be improved by the proper choice of electrodes [32]. 
The quality of the piezoelectric film is another major factor influencing the coupling in a BAW resonator. A rough bottom electrode significantly degrades coupling due to processing reasons. Thus, the smoothening of the bottom electrode is also important. For an SMR with metal layers in the Bragg reflector, a parasitic capacitive coupling with the contact pads will reduce the coupling coefficient further. This parasitic coupling can be eliminated by patterning of the Bragg reflector as proposed in [6], [34]. An alternative approach is fabricating the SMR on a dielectric reflector [10], [35] and [36].

In summary, the reflector stack and most importantly the electrodes stack have a strong influence on the effective coupling coefficient in a BAW device. A properly designed reflector stack can enhance coupling while a poorly designed stack will degrade coupling [15].

\subsubsection{The quality factor ( $Q$ factor)}

The quality factor ( $Q$ factor) is a measure of the energy dissipation within the system, indicating how well mechanical energy input to the resonator remains confined there during the oscillatory motion. In the resonator, the energy oscillates between kinetic and potential forms, and during these cycles, some energy is inevitably wasted due to internal friction and other loss mechanisms (see section 2.4). For a mechanical resonator, the $Q$ factor is indicative of the rate at which energy is being dissipated and is generally defined as [12]:

$$
Q=2 \pi\left(\frac{\text { Stored energy }}{\text { Lost energy per cycle }}\right)
$$

With a force applied at its resonance frequency, a resonator with an infinitely high $Q$ would vibrate with non-decreasing amplitude, never losing energy to its surroundings, and continue to vibrate indefinitely once the applied force is removed. Unfortunately in a practical resonator, there are some losses associated with the device and hence the achievable $Q$ is limited. Consequently, a high $Q$ is one of the most desired parameters in BAW resonator design as it indicates a low rate of energy dissipation. High $Q$ resonators when used in the filters offer a high transmission in the pass band.

There are several methods to extract the $Q$-value of a BAW resonator from the measurements [10]. One practical approach is the phase derivative method to extract the $Q$-factor from the steepness of the phase $(\varphi(f))$ curves according to [24]:

$$
Q^{\varphi}=\left.0.5 \cdot f_{0} \cdot \frac{d \varphi(f)}{d f}\right|_{f=f_{0}}
$$

where $f_{0}$ is the frequency of interest. 
Another extraction method is the traditional 3-dB bandwidth method to determine the bandwidth $\Delta f$ at the $-3 \mathrm{~dB}$ level of the admittance or impedance curves according to [11]:

$$
Q^{\mathrm{BW}}=\frac{f_{0}}{(\Delta f)_{3 d B}} .
$$

Although the formulas for calculating the $Q$ factor are well defined, obtaining a reliable $Q$ from experiments is challenging [15], [28]. Methods for determining the $Q$ are quite sensitive to the frequency step size in the measured range [10], [11], [25]. Moreover, any spurious modes or other non-idealities at the measured frequency greatly complicate a direct $Q$ calculation from the measured $S$-parameters [15]. For a qualitative study of $Q$ values, either eq. (2.16) or eq. (2.17) can be used. The choice of the method to use depends on the application as well as user preference [27].

For the experimental extraction of the $Q$ factor, a much more robust method is to fit the impedance curve using the mBVD model [13], [15]. By using such a model, the derivation of the $Q$ factor simply becomes a matter of calculating the stored energy and the dissipated energy per cycle (see also eqns.(2.11)-(2.12)) from the input impedance of the circuit defined in the section 2.2.2. However, the accuracy of this approach depends on how this fitting is done [13], [25], [28]. A comparison of the $Q$ values calculated by various methods is presented in [10]. The $Q$ values predicted by the phase derivative method and the traditional 3-dB bandwidth method yield similar results. These values are higher than the $Q$ values extracted from the mBVD fit. However, the results are comparable to the other methods. The comparison of $Q$ values obtained from various resonators is legitimate only when the same method has been employed to compute it.

The $Q$-value at resonance or anti-resonance depends on the series resistance or some shunt conductivity, either the resonance or the anti-resonance will show the larger $Q$ value. Some authors propose [6] to define an acoustic $Q$-value which is equivalent to the maximum of those two values. In electrical measurements it is straightforward to distinguish between acoustic losses and electric losses, because in a frequency sweep electric losses can be seen even far away from the acoustic resonance frequency $\left(f_{\mathrm{R}}\right.$ or $\left.f_{\mathrm{A}}\right)$ where acoustic losses no longer play a role. The $Q$-value at the resonance frequency, $Q_{R}$ is lower than at the anti-resonance frequency $Q_{\mathrm{A}}$, since there's a strong influence of electrical (ohmic) losses for the former, which will be addressed in section 2.4. Hence, although there is an overall improvement in the $Q$-value of BAW resonators, it is mainly observed in $Q_{\mathrm{A}}$ and not in $Q_{\mathrm{R}}$. Therefore, in general the anti-resonance $Q_{\mathrm{A}}$ is the best parameter to look at while investigating acoustic losses in a BAW device [13], [25]. $Q_{\mathrm{A}}$ is mainly related to the mechanical losses rather than the electrical losses and hence used to quantify the influence of the acoustic reflector on the performance of the resonators [33].

\subsection{3 $k_{\text {eff }}^{2}$ and $Q$}

For the practical applications, both a sufficiently high coupling and $Q$-values are the goal [6]. However, there is a trade-off between these parameters [10]. Therefore, to judge 
the performance of a BAW technology, a so-called figure of merit (FOM) has been introduced:

$F O M=k_{\text {eff }}^{2} \cdot Q$

Note that $k_{\text {eff }}^{2}$ is not a function of frequency while $Q$-value is a function of frequency and therefore FOM is also a function of frequency [10], [37]. Hence FOM is more commonly used in filter design than in the resonator design [38].

Device designers can trade off $k_{\text {eff }}^{2}$ against $Q$ factor depending on the application. A small sacrifice in $k_{\text {eff }}^{2}$ gives a large boost in $Q$ value [10]. $k_{\text {eff }}^{2}$ can be enhanced by choosing a high acoustic impedance electrode, and can be also traded off with other parameters such as electrode thickness and a thicker passivation layer [11]. In the case of SMRs, loading the reflector stack with a high acoustic impedance metal also seems to improve the coupling coefficient [35], [36].

\subsection{Loss mechanisms and $Q$ factor}

The loss mechanisms in thin film BAW resonators can be divided into two major categories: electrical and acoustical losses [40]. The acoustical losses mainly include acoustic leakage to the substrate, laterally escaping waves; though viscous losses and wave scattering are also sometimes referred to as acoustic attenuations [27], [28]. Except for acoustic leakage to the substrate (discussed in detail in section 2.4.1), all other loss mechanisms are associated with both FBARs and SMRs.

Electrical losses are caused by resistance of the resonator electrodes and leads connecting resonators and bonding/probing pads. Utilizing low resistivity materials such as $\mathrm{Au}$ or $\mathrm{Al}$ in the electrodes with high enough thickness reduces ohmic losses, but resistivity must be co-optimized with other properties such as the coupling coefficient and this easily leads to trade-offs [28],[32]. Dielectric loss and eddy current losses are the other reported electrical loss paths [13].

Another possible acoustic loss path is the energy loss by laterally leaking waves [27]. The existence of this type of loss is visibly observed in the interferometer measurements of BAW resonators. However, the amplitudes of these waves are considerably smaller than the amplitudes in the active area of the resonator caused by the longitudinal waves. The reason for their excitation is most likely the discontinuity at the resonator edge. Experiments have shown that lateral wave leakage is not a dominant loss mechanism in SMRs in the $Q$-regime up to 2500, however might become significant above this threshold. It has been shown experimentally [40] that lateral energy leakage can indeed be prevented by appropriate measures. The laterally leaking waves when trapped within the electrode boundaries lead to additional unwanted spurious resonances are formed by standing waves [13], as explained in section 2.6.

Viscous losses are intrinsic to the materials used in the devices. No material is perfectly elastic; only some of the energy stored in a visco-elastic system is recovered upon 
removal of the load. The remainder is dissipated in the form of heat, causing a loss of energy for the acoustic vibration [27]. Apart from the choices of materials, not much can be done about these losses other than optimizing the process parameters. Among the acoustic materials typically used in BAW devices, $\mathrm{SiO}_{2}$ and $\mathrm{Al}$ have largest losses, while $\mathrm{AlN}, \mathrm{Mo}$, and $\mathrm{W}$ are all rather low-loss materials [41]. For the visco-elastic damping constants literature values [42] are typically used. But it is a difficult work to get any reliable loss parameters for thin-film material from experiments or from the literature [6]. The viscoelastic losses become significant in SMR-BAW devices once the $Q$-regime is above $\sim 3,000$ [13].

Scattering losses occur due to material layer imperfections and surface or interface roughness [13]. The main loss mechanism is the redirection of vertically moving acoustic energy towards lateral directions. This causes the waves to leave the active resonator region and dissipate either in the device substrate or in the regions surrounding the device laterally. However, it has been shown that typical processing related nonuniformities do not affect the quality factor of the resonator because the acoustic wavelength is greater than the non-uniform layer thickness variations [40].

\subsubsection{Acoustic leakage through the reflector stack}

As mentioned in section 2.1.1, SMR devices contain an acoustic reflector for acoustical isolation from the substrate. However, the quarter wavelength $(\lambda / 4)$ Bragg reflector is optimized for one particular wavelength i.e. the wavelength of the longitudinal main mode at the resonant frequency. Hence the Bragg reflector cannot isolate the acoustic waves of other wavelengths from escaping into the substrate. Due to the vibration or deformation of the piezoelectric layer, acoustic waves with other wavelengths are also generated and hence some acoustic energy will be leaking through the reflector stack into the substrate. The energy that leaked into the substrate cannot be recovered; this loss mechanism significantly reduces the quality factor of the resonance [13].

The dominant loss mechanism in the traditional quarter wavelength SMR is the loss caused by shear waves (which is explained further in this section) generated in the device and transmitted through the mirror. It is a well established fact that the $\lambda / 4$ reflector stack has the best acoustical isolation from the substrate when only the chosen wave type (here longitudinal) is considered. However, as the shear wave velocity being about half that of longitudinal wave velocity [44], a quarter wavelength stack designed for the reflection of longitudinal waves meets the full transmission condition $(\lambda / 2)$ for shear waves. Therefore the conclusion without any additional analysis is: if shear waves are generated in the device, they are transmitted readily through the mirror, and a corresponding $Q$-loss will be observed. This loss can indeed be very large for even small amounts of shear waves involved. Although the reported shear amplitudes are approximately a factor of 10 times smaller than that of the amplitude of longitudinal waves, they still constitute a significant contribution to the losses and results in a decrease in the resonator $Q$, effectively limiting the $Q$ to the regime of $<700$ [13],[28].

The origin of the shear waves can be attributed to three probable reasons [43]. Firstly, at the edges of a resonator, certain acoustic edge conditions for the boundaries between the 
active area and the outside area must be fulfilled. If these boundary conditions are not matched, shear waves are generated at the circumference of the resonator. The real device is indeed a 3D-structure having lateral boundaries and hence these boundaries can easily be a source of shear waves. Secondly, if the piezoelectric layer of the resonator has tilted grain boundaries, then a vertical field may lead to the launch of shear waves in the piezoelectric layer. Thirdly, longitudinal waves moving in a direction not exactly perpendicular to the substrate plane can get converted to shear waves at the interface of different layers.

The solution to the shear wave leakage problem is to design the mirror in such a way that both the longitudinal and shear waves are reflected. The improvement of $Q$ factor by minimizing the shear wave energy leakage throughout the reflector stack was first reported by Infineon's BAW group [24], [43]. They have presented a co-optimized reflector stack providing good reflection for both shear and longitudinal waves. Recent reports from various groups among the BAW (SMR) community [33], [39] confirm that careful co-optimization of the reflector for longitudinal and shear waves significantly boosts the $Q_{\mathrm{A}}$. Without consideration of the shear wave reflection, the quality factor of SMRs was limited to about 700. In this case the losses seem to be dominated indeed by the acoustic reflector leakage due to shear waves. Careful reflector design on the other hand boosted the resonator $Q_{\mathrm{A}}$ up to 2000 [44].

It should be mentioned that this co-optimization of the reflector stack for shear waves goes hand-in-hand with sacrificing some piezoelectric coupling, since usually layers in the reflector close to the resonator have to be made thicker. This in turn causes a larger part of the stress field to reside outside the piezoelectric material, thus reducing the coupling [13].

As we have discussed, the shear waves can constitute a major loss mechanism for BAW resonators. Although many co-optimized reflector stacks [33],[35],[36],[43],[44] have been reported, none of them discusses a systematic design procedure for the optimization. The prime investigations of this thesis [39] are on systematically optimizing the reflector stack for both longitudinal and shear waves. A detailed analysis of our reflectors stack design is presented in chapter 3.

\section{Quality factor vs. transmission}

As we have been discussing, the quality factor ( $Q$ factor) is a measure of losses in the system. The very basic definition (eq. (2.15)) states that the $Q$ factor is inversely proportional to losses in the system. Hence an improvement of $Q$ factor can be achieved by minimizing the losses.

If the losses are for example only in the form of energy transmitted through the reflector stack to the substrate described by transmission $T$, the $Q$-value takes the form [41]: 
$Q=2 \pi\left(\frac{E_{\mathrm{tot}}}{T \cdot E_{\mathrm{tot}}}\right)=\frac{2 \pi}{T}$,

where $E_{\text {tot }}$ is the total stored energy. This way one can translate the transmission of the waves in the reflector stack into a $Q$-value. The former can be obtained using the Mason transmission line model as described in section 2.2.1. The simple Mason model calculation is a 1D-treatment and there exists no mechanism for generating any shear waves in it. By using Mason's model to simulate the longitudinal and shear wave transmission of a given reflector stack configuration, the resulting limitations to the resonator quality factor can be calculated [13].

Assuming various loss mechanisms as discussed, from the nature of the $Q$-value being inversely proportional to relative losses, it follows that [13]:

$$
\frac{1}{Q_{\mathrm{tot}}}=\sum_{l} \frac{1}{Q_{l}}
$$

where $Q_{l}$ is the $Q$ value associated with the loss mechanism $l$ and hence the summation is over different loss mechanisms.

Now, inspecting a single loss mechanism such as the transmission through the reflector stack, we can interpret its resulting $Q$-value as the one that the device would exhibit in case all other loss mechanisms where much smaller in magnitude. We can thus interpret the minimum transmission resulting from the reflector simulations as the inverse of a 'reflector limited $Q$-value' [41]. This is the approach adopted in this thesis, thus relating the wave transmissions of the reflector stack directly to the $Q$-factor.

The acoustic leakage through the reflector stack being the dominant loss mechanism in SMRs, a significant improvement in $Q$ values can be achieved by minimizing this loss. The approach of Marksteiner et al. [24] to model both longitudinal and shear transmission in an acoustic reflector is by separately calculating longitudinal and shear transmission in 1-D, and then assuming a constant fraction of the energy to be in the shear waves. Thus they calculate a composite acoustic $Q$-value combining the losses due to longitudinal and shear wave leakages. With an assumed value of $1 \%$ of the total energy converted to shear waves, the total quality factor drops significantly (one order) despite the seemingly small amount of energy associated with the shear waves. A recent work [33] also reports an increase in quality factor from 200 to 2800 by minimizing the shear losses to the substrate. The authors of that work also present the experimental evidence that the variations of the $Q$ factor follow the trend of shear transmission rather than longitudinal transmission with acoustic reflectors of different layer thicknesses. They empirically estimate a lower value of $0.05 \%$ as the amount of energy stored in the shear modes. Unfortunately there are no reports of the amount of energy stored in shear waves from experiments.

Looking at the $Q$-factor for the transmission analysis of the reflector stack is a successful method in practice; however, it is an indirect method. Alternative methods exist [45] to 
analyze the longitudinal and shear transmissions. A direct experimental method is the analysis by the laser interferometric measurement technique [46]. In this method, mirror transmission characteristics are analyzed by the vibration amplitude measured by the interferometer both on the surface of the resonator and at the reflector-substrate interface. But this requires devices to be fabricated on a glass substrate [47]. Because of the practical difficulties to fabricate the devices on a glass substrate and due to unavailability of the interferometer set-up, we chose to follow the $Q$-factor analysis for evaluating the performance of the reflector stack as presented in chapter 6 .

\subsection{The acoustic dispersion}

In section 2.2 we introduced the Mason model, describing the $1 \mathrm{D}$ behavior of a BAW resonator. For a one dimensional device, this model is fully descriptive for the loss due to longitudinal wave energy leaking to the carrier substrate. In section 2.4 we stated that for resonators with finite lateral dimensions, longitudinal waves as well as shear waves are present in the resonator. In this section we will detail how the device performance is influenced by the existence of both of these waves.

This section is organized as follows: section 2.5.1 introduces the dispersion curve. Section 2.5.2 discusses the concept of eigenmodes or guided modes for devices with finite dimensions and further introduces the representation of eigenmodes in the dispersion curves. Section 2.5.3 addresses the construction of dispersion curves for bulk media and multilayered systems. In this section we will also concentrate on one particular guided mode which is very close in nature to the 1D solution of infinitelysized resonators. The dispersion curve for this mode is one of the central themes in BAW development. Finally, section 2.5.4 discusses the types of dispersion.

\subsubsection{Introduction}

The dispersion curve in its very basic form is a plot of frequency $f$ against the wave number $k$ as shown in Figure 2.6. The wave characteristics are defined by $k$ and $f$ and they are connected to the wave velocity $v$ according to $v=2 \pi f / k$ [48]. For a non-dispersive medium, i.e. a medium where the wave velocity is independent of frequency, the relation is obviously linear and the slope at any point in the line on the plot is a measure of wave velocity. In the case of dispersive media, the relation between $f$ and $k$ is no more linear indicating the frequency dependency of wave velocity. In the case of optics, the dispersion curve is continuous while in the case of mechanics (acoustics) discrete points constitute the dispersion curve.

The acoustic dispersion relation plays a key role in the design of BAW resonators in understanding and controlling the lateral propagation characteristic of the waves. In BAW resonators, dispersion curves give the information about the kind of waves characterized by the lateral propagation constant $k_{\mathrm{x}}$ which can be supported by the resonator at a given frequency [28]. Lateral waves arise in a layer stack because of the fact that each layer, when excited with any vibration is essentially an acoustic waveguide with guided waves having energy flow mainly along the direction of the 


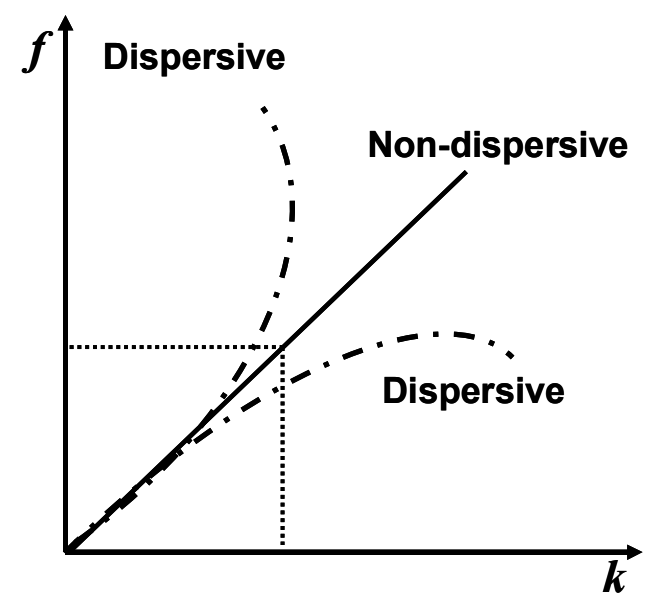

Figure 2.6: $\quad$ A plot of frequency $f$ against the wave number $k$ for a non-dispersive (solid line) and dispersive (dashed line) medium. The slope f/k at any point on the lines is a measure of the wave velocity. In the case of dispersive media, the relation between $f$ and $k$ is no more linear indicating the frequency dependency of wave velocity.

guiding configuration i.e. the lateral direction [49]. In short, lateral waves are guided waves in the lateral direction in which the particle displacement is in the vertical direction $(z)$ and the wave propagation is in horizontal $(x)$ direction.

A thin film bulk acoustic wave resonator consists of a stack of different layers, which are dispersive for acoustic waves, with different acoustic properties. In a bulk medium, three important vibration modes propagate: a longitudinal mode and two differently polarized shear modes [12], [50]. The longitudinal mode is characterized by particle displacement in the direction of the propagation, whereas the shear modes consist of particle displacement perpendicular to the direction of propagation with no local change of volume. The propagation characteristics of the three bulk modes depend on the material properties and propagation direction respective to the crystal axis orientation [12], [49].

In this thesis, we limit the discussion to one longitudinal and one shear mode for simplicity. When a propagating bulk wave encounters an interface with another material with different material properties, like in optics, transmitted and reflected waves are generated. In such an event, a pure bulk mode is reflected as a pure bulk mode of the same type only in exact normal incidence. Generally, the stress and particle displacement continuity conditions at the interface require an incident wave to be reflected and transmitted as a combination of all bulk modes propagating in different angles. The phenomenon is called mode conversion and is governed by Snell's Law [41], [51].

Let us consider two dimensional structures having one vertical dimension $(z)$ and a single horizontal dimension $(x)$. In a structure formed of a finite number of stacked plates like in SMRs, pure bulk modes can propagate and form standing wave resonances in the $(z)$-direction perpendicular to the layer interfaces. If, however, the wave propagation direction of any of the bulk wave components, defined by the wave vector 

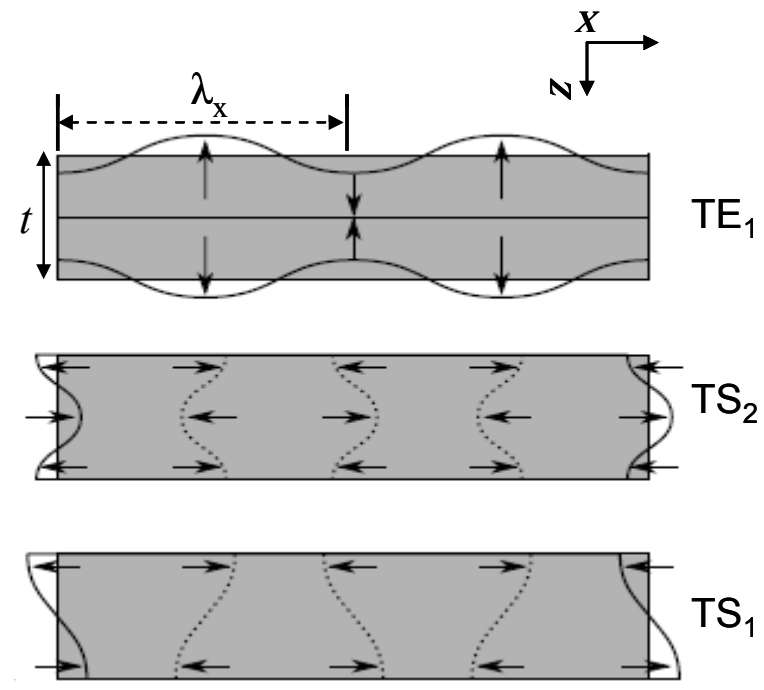

Figure 2.7: $\quad$ Illustration of some plate wave modes for a single free standing plate: the 1st thickness extensional (TE1), the 2nd thickness shear (TS2) and the 1st thickness shear (TS1), from top to bottom, respectively. Two full lateral wavelengths are shown for each mode. In this example, the frequency increases from bottom to top however, the order of TE1 and TS2 depends on the Poisson ratio of the plate material [41].

$k$, deviates from the perpendicular one, mode conversion according to Snell's law takes place at every interface. It turns out that with certain combinations of propagation directions, repeating mode conversion of reflections at the interfaces reproduces the original field in a periodical manner in the lateral $x$-direction [28]. These kind of wave modes propagating in the $x$-direction are called plate wave modes or Lamb waves [49],[53], some basic types of which are illustrated in Figure 2.7 for a single plate.

The laterally finite BAW resonator operation is actually defined by the characteristics of these Lamb waves because such a resonator practically never can really resonate in a pure bulk wave mode. The presence of laterally propagating waves in vertically stacked layer structures has the important consequence that the operation of the intended vertical-mode resonators becomes sensitive to the lateral boundary conditions and geometry of the device as reflections of these waves from lateral discontinuities occur [41]. In short, the resonance frequency is affected by lateral dimensions as well. Therefore, it is of crucial importance to be able to study the lateral propagation characteristics, i.e. the plate wave dispersion relations, in the BAW devices in order to be able to design high performance devices.

\subsubsection{Eigen mode concept for dispersion curves}

Two-dimensional device behaviour of the BAW resonator in the $x-z$ plane is usually explained in terms of laterally propagating plate waves in the $x$ direction. Assume a rectangular resonator with edge lengths $W$ and $L$ in $\mathrm{x}$ and $\mathrm{z}$ direction respectively. First we assume Dirichlet boundary conditions ( $u=0$ at the edges). The eigenmodes*, indexed

\footnotetext{
* Eigenmodes are natural modes of vibration in a system.
} 
$(m, n)$, simply have the displacement profile of the form $\cos (2 \pi m x / W) \cos (2 \pi n y / L)$ with lateral wave number $k_{m, n}$ holds [4],[54] :

$k_{m, n}=\pi \sqrt{\frac{m^{2}}{W^{2}}+\frac{n^{2}}{L^{2}}}$,

where $m, n=1,2,3 \ldots$

The eigenmodes are thus time-independent solutions described by a displacement profile and an eigenfrequency associated with it. One can read the associated eigenfrequency $f_{m, n}$ from the dispersion curve, obtained from interferometry or 2Dsimulation. The response of the resonator to excitation by electric field at an excitation frequency can be written as a superposition of eigenmodes.

For a practical (finite) BAW resonator, dispersion curves are formed by discrete points on a smooth continuous line [55], [56]. The discrete points on the dispersion curves that can form trapped standing wave resonances within the resonator width $W$ can be determined in terms of lateral wave number according to [54],[56]:

$k_{x}=\frac{m \cdot \pi}{W}$,

As discussed, the frequencies of these modes, $f=f\left(k_{x}\right)$, can be determined from 2D eigen frequency simulations to plot a dispersion curve.

\subsubsection{Construction of dispersion curves}

An example of a dispersion curve of the TE1 Lamb wave mode in a BAW resonator is shown in Figure 2.8. While the wave vectors of bulk waves have different lengths and directions in layers of different materials, they all share the same $\mathrm{x}$-component and thus lateral wavelength. The positive values of $k_{\mathrm{x}}$ stand for real valued wave number, but the negative side of the axis is used to represent imaginary values of $k_{\mathrm{x}}$. The lateral wave number $k_{\mathrm{x}}$ is real for a laterally propagating mode and is imaginary for evanescent waves representing non-propagating, non-energy carrying vibration. The evanescent waves have an important role in designing proper boundary conditions in actual devices.

We now explain the formulation of the dispersion relation starting with an isotropic layer, then introducing anisotropy and further referring to a general multilayer case. Making use of the transverse resonance principle [49] which states that a guided wave is a traveling wave along the waveguide axis $\mathrm{x}$ and resonant standing wave in the $\mathrm{z}$ direction, for a single isotropic layer, the (type I) dispersion relation can be represented by:

$k_{x}=\sqrt{\left(\frac{\omega}{v}\right)^{2}-\left(\frac{n \cdot \pi}{t}\right)^{2}}$, 


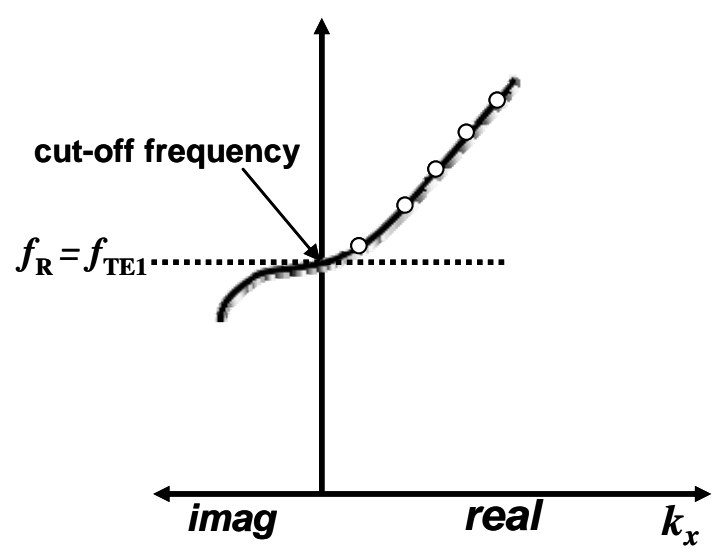

Figure 2.8: $\quad$ Illustration of a dispersion curve. Real $\left(k_{x}\right)$ represents the lateral wave number of the propagating wave and imag $\left(k_{x}\right)$ represents that of the evanescent wave. The dashed line represents the $1 D$ resonant frequency $f_{R}(k=0)$ which is the cut-off frequency at which the wave changes from propagating to non-propagating. The spacing between the discrete points in the dispersion curves depends on the width of the lateral dimension (eq.(2.22)).

where $v$ is the acoustic velocity of the wave considered, $t$ is the layer thickness and $n=0,1,2,3 \ldots$

Mode $1(n=1)$ has $k_{\mathrm{x}}=0$ at the series resonance frequency. Therefore at this frequency, the lateral wavelength is infinite and the mode 1 is the same as the 1D mode. Hence, TE1 indicates the thickness extensional mode for $n=1$ and TS2 indicates thickness shear mode for $n=2$.

Assuming now anisotropy in the piezoelectric layer, if $v_{L}$ is acoustic velocity of longitudinal waves and $v_{\mathrm{S}}$ that of the shear waves in the material, substituting $k_{\mathrm{x}}=0$ in eq.(2.23) allows us to calculate the frequency of TE1 and TS2 modes as :

$$
\begin{aligned}
& f_{T E 1}=\frac{n \cdot v_{L}}{2 \cdot t}=\frac{v_{L}}{2 \cdot t}, \\
& f_{T S 2}=\frac{n \cdot v_{S}}{2 \cdot t}=\frac{v_{S}}{t} .
\end{aligned}
$$

Eq. (2.24) implies $f_{\mathrm{TE} 1}=f_{\mathrm{TS} 2}$ only when $v_{\mathrm{L}} \approx 2 \cdot v_{\mathrm{S}}$. In practice, $v_{\mathrm{L}}$ and $v_{\mathrm{S}}$ are interconnected according to [4], [12], [44]:

$$
\frac{v_{\mathrm{S}}}{v_{\mathrm{L}}}=\sqrt{\frac{2 \sigma-1}{2 \sigma-2}} .
$$


In a general multilayer case, the dispersion relations are solved by matrix methods, the input parameters being the layer thicknesses and material properties of the various layers comprising the resonator stack [51],[52]. This is because the acoustic waves are not necessarily confined in the piezoelectric layer; there is some energy leakage to the other layers.

In a loss-less layered structure surrounded by vacuum (for e.g. FBARs), the plate modes have either purely real or purely imaginary $k_{x}$. However, in the case of SMRs in which the wave mode radiates energy into the reflector stack below, the lateral wave number of the plate modes becomes complex. These modes are no more true loss-less vibration modes but propagating modes which are damped thus representing a lossy wave. Handling of complex wave numbers in the dispersion calculation is desirable but adds a significant amount of complexity in the calculation. Complex wave number simulations for a multilayer case have been employed in [57]. In this work, we have limited to the real loss-less TE1 wave mode for determining the dispersion types discussed below. The analysis of TE1 mode is appropriate for determining the dispersion relation of SMRs with a high-performance, shear and longitudinal wave co-optimized reflector.

\subsubsection{Types of dispersion}

Before discussing the dispersion types, it is important to know the concept of energy trapping. This concept enables the lateral effects in a BAW resonator to be studied in more detail. Energy trapping phenomenon was first discussed for quartz resonators by Shockley et al. [58]. The principle of energy trapping states that for a layer stack having the dispersion behavior as given in Figure 2.9 (b), the cut-off frequency of the external region $f_{\text {ext }}$ is higher than the cut-off frequency of the internal region $f_{\text {int. The cut-off }}$ frequency is defined as the frequency where the lateral (Lamb) wave number is zero. In other words, the cut-off frequency is the frequency at which the mode changes from propagating to non-propagating (see Figure 2.8) [28].

This energy trapping is readily accomplished by the structure as can be seen from the drawn cut-off frequency diagram (Figure 2.9 (a)). Here the frequencies where the wave number is imaginary are denoted by the gray shading. For frequencies between $f_{\text {ext }}$ and $f_{\text {int }}$ the wave is trapped or confined to the internal region of the resonator. As stated in [58] that if the external region in a BAW resonator $(x<-W / 2$ and $x>W / 2)$ presents a higher cut-off frequency than the exciting frequency, the resulting vibratory energy is essentially confined to the active region of the resonator $(-W / 2<x<W / 2)$ with an energy distribution decreasing exponentially with distance away from the resonator. This exponential decay is not per definition associated with energy loss and traps the oscillating energy within a confined region.

Depending on the relative position of longitudinal main or fundamental resonance mode (TE1) and the second shear resonance mode (TS2), the dispersion relation of a BAW resonator is classified into two types: If $f_{\mathrm{TE} 1}>f_{\mathrm{TS} 2}$ the dispersion is type I, and type II otherwise [44]. Figure 2.10 shows the dispersion curves for two different types including both the thickness and shear branches. It must be pointed out that the first thickness 


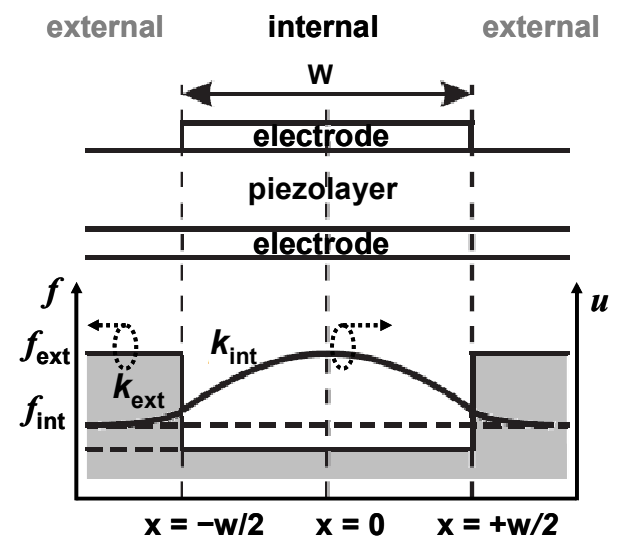

(a)

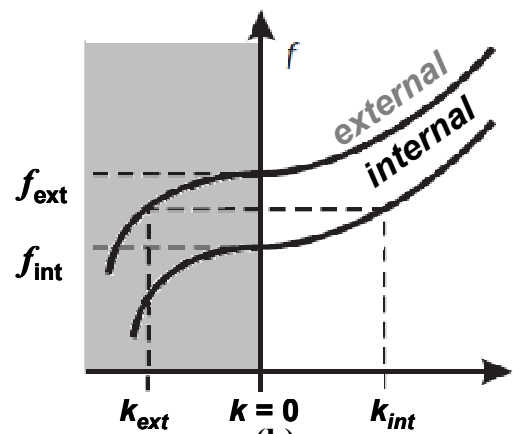

(b)

Figure 2.9: (a) Geometry and cut-off frequency diagram of a traditional resonator indicating the internal and the external regions of the device (b) dispersion characteristics of the thickness extensional (TE) mode in the internal and external regions of the resonator [28].The internal region is also known as the active area of the resonator. $u$ is the vertical displacement in the lateral direction.

shear TS1 mode occurs at very low frequencies, making the effects at the fundamental resonant frequencies of the BAW resonator almost negligible and this is therefore not shown in the figure. However, the TS2 mode is closer to the fundamental thickness mode (TE1) frequency and hence is considered for determining the dispersion type. In some representations of type I and type II resonators [10], the curve referring to TS2 mode bends downwards. This indicates a negative group velocity (see [12], [49], [48]) for the TS2 waves which is due to the characteristic (material parameters) of the layers in the stack [59].

A very important feature (as in Figure 2.10) is that in the case of type I dispersion, $f_{\text {TE1 }}$ is related to the dispersion branch of positive slope whereas in type II dispersion it is

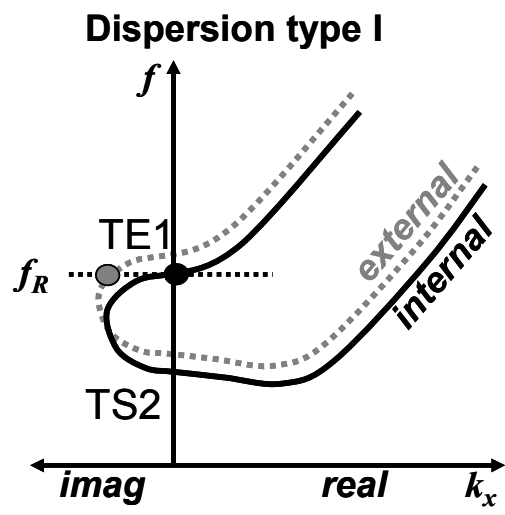

(a)

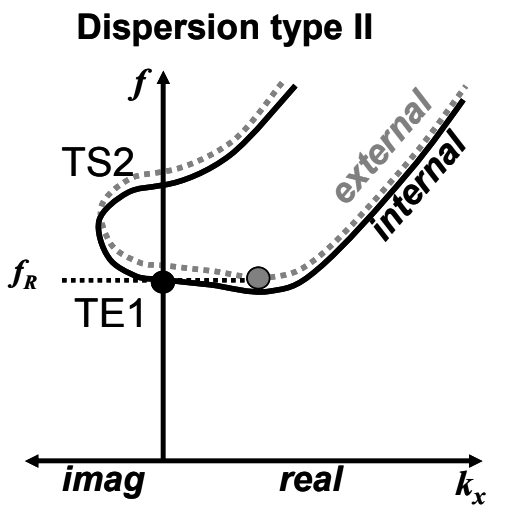

(b)

Figure 2.10:

Dispersion curves showing two types of dispersion (a) Type I and (b) Type II. In (a), the second shear harmonic resonance (TS2) is above the longitudinal main resonance (TE1) $\left(f_{T E 1}<f_{T S 2}\right)$ whereas in (b), $f_{T E 1}>f_{T S 2}$ confirming type I dispersion. In (a), $f_{T E 1}$ is related to the dispersion branch of positive slope whereas in (b) it is related to that of the negative slope. Note that the wave vector $k_{x}$ in the external region is imaginary (represents an evanescent wave) at the resonant frequency in type I and in the case of type II, it is real (represents a propagating wave) in the external region. 
related to that of the negative slope. A positive slope of the TE1 mode in type I dispersion indicates a positive group velocity for the lateral waves where as the negative slope in type II dispersion indicates a negative group velocity for the lateral waves [28],[44].

In the case of a single isotropic plate approximation, the relative positions TE1 and TS2 depend only on the material parameters. As mentioned before, the physical parameter relating the longitudinal wave velocity to the shear wave velocity in a given material is the Poisson ratio. From eqns.(2.24) and (2.25), it can be seen that, depending on the Poisson ratio, the shear wave resonance frequency can be either above or below the longitudinal wave resonance frequency [44]. Mindlin and Medick derived in their analysis of extensional vibrations in isotropic elastic plates, the Poisson ratio $\sigma$ is the critical parameter determining the slope of the dispersion curves [60]. The influence of $\sigma$ on the dispersion type of a laterally infinitely extended isotropic plate is presented in [44] . For that type of resonator, the dispersion type changes at a value of about 0.33 for the Poisson ratio, thus longitudinal and shear resonance are at the same frequency for this value $\left(v_{\mathrm{L}} \approx 2 \cdot v_{\mathrm{S}}\right)$. For $\sigma>0.33$, the dispersion is type I and for $\sigma<0.33$, the dispersion is type II.

Aluminum Nitride (AIN) being a type II material $(\sigma=0.25[44])$, the resonators based on AlN without any precautions (e.g. an FBAR) would usually end up in a type II behavior [10], [27], [43], [44]. However it is possible to achieve type I response in an SMR by proper modification of stack layers [43], [44], [61], [62]. It is critical to have type I response for the suppression of spurious modes by the application of a frame region which is discussed in the next section.

\subsection{Spurious resonances and their suppression}

One of the direct consequences of lateral energy trapping discussed in the former section, is the occurrence of spurious ripple due to lateral standing Lamb waves in the active area of the resonator [63]. Spurious resonances are unwanted resonances or ripples in the electrical characteristics of the BAW resonator which adversely affect the filter characteristics and also affect the extracted $Q$ value.

The lateral standing wave arises due to the finite lateral dimensions of the resonator structure. The origin of the spurious resonance can be understood as follows. Around resonance there is strong acoustic vibration in the electroded or internal region. The region outside the resonator is hardly moving. If a laterally propagating wave is excited it will be bounced from the electrode to electrode edge due to acoustic mismatch at the edges. For particular frequencies it forms standing waves. This happens if the distance from edge to edge of the electrode is an odd number of half wavelengths, due to the free boundary condition (zero stress) at the edges of the electrodes. This standing acoustic wave changes the amount of charge at the electrodes, compared to the situation without the standing wave. A change in charge means a change in current and thus the admittance. With the normal electrode edge conditions, the charge density is close to zero at the edges. Consequently at those frequencies where the electrode region fits an 


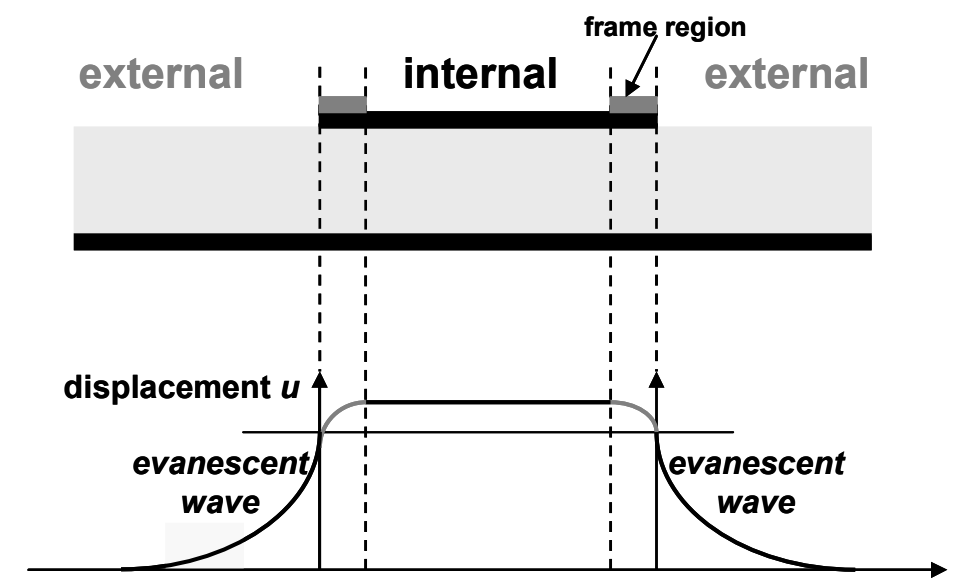

Figure 2.11: $\quad$ Schematic cross-section of a BAW resonator including a frame region for spurious mode suppression (top) and the associated displacement profile (bottom) in the case of type I dispersion [66].

odd number of half wavelengths, there is a peak in the impedance curve which is referred to as a spurious resonance [28], [63].

The degradation of the performance of the BAW resonator due to these spurious resonances is discussed in [64]. This is basically due to the fact that part of the energy contained in the fundamental thickness mode leaks to the lateral modes. However, it is more evident when the BAW resonators are in a filter configuration leading to a strong ripple in the pass band transmission. In fact the vibrations associated with these resonances can be a major source of energy loss. In order to achieve higher $Q$-values, those losses must be confined and hence spurious resonances should be suppressed [15].

To suppress the impact of spurious modes, three different methods have been proposed [65]. The first technique termed as apodization is designing the top electrode with nonparallel edges so that no odd number of half wavelengths fit over the electrode length at the same frequency. The second method is to engineer the resonator to have a very flat dispersion profile where all the spurious resonances would be spaced very close to each other in frequency, practically merging together in the electrical response. The dispersion engineering is quite demanding to realize although in this case spurious resonances becomes less pronounced. However, these two methods do not suppress the excitation of spurious modes, but actually minimize their impact on the electrical characteristics. This usually also results in a lower $Q$-value. Kaitila et al. [63] proposed to include a specific frame region (or border region) to match the boundary conditions for a homogeneous excitation in the active area and the exponentially decaying tails in the area outside the resonator as shown in Figure 2.11. This method is the only one that tackles the physical origin of spurious modes; i.e. prevents spurious modes from being excited, and therefore enables spurious free resonators with maximum $Q$-value [66]. 
As a precondition for the above referenced "frame region" concept, the dispersion relationship of the layer stack should be of "type I". Figure 2.10 shows that for a type I resonator, the cut-off frequency in the external region is higher than that of the internal region. Hence, at $f_{R}$, the lateral wave number $k_{x}$ for the external region becomes imaginary indicating an evanescent wave. This criterion should be kept in mind while optimizing the acoustic reflector stack to provide high reflection for both longitudinal and shear waves discussed in section 2.4.1. In fact this limits the freedom in designing an acoustic reflector significantly [15]. Although most of the reported literature concentrates on the frame region of dispersion type I resonators for spurious suppression it should be noted that nothing, in principle, excludes applying the idea also to type II devices. In this case to achieve the real wave number in the border region it needs to be designed thinner than the active area. However, so far there has no experimental work been published regarding spurious mode suppression using the outlined scheme in type II device [28]. Some problems associated with energy trapping using this scheme are discussed in [44].

In summary, for a type I resonator, the frame region can be implemented directly. For type II (typical AIN based) resonators, a recessed frame can be applied or dispersion curves should be flipped to type I for applying the frame region concept [10]. The flipping of dispersion relation by proper modification of the stack layers in SMRs is discussed in [43], [44], [61], [62]. In this thesis, a rule of thumb [67] for flipping the dispersion curves applicable for both FBARs and SMRs is presented in chapter 4 .

\subsection{Chapter summary}

In this chapter an overview of the basics of BAW device physics is presented. The concept of the BAW resonator is introduced and the two main BAW resonator configurations are explained. The piezoelectric effect has been described as a property of certain materials in which applied mechanical force results in a generated electric field in the structure and vice versa. The impedance curve of a BAW resonator and its important parameters is explained. The piezoelectric effect is responsible for the resonance peaks in the impedance curve.

The relevant models for the BAW resonators have been presented. The 1-D Mason model is described which uses the transmission line concept in which the piezoelectric layer is a three port network having two acoustic ports and one electric port. The model describes the electrical behavior for any mechanical load condition. The Butterworth Van Dyke model has also been discussed which is a circuit model consisting of lumped elements. The lumped nature of the circuit makes it suitable for parameter extraction and design studies.

The key parameters for the BAW resonators, the quality factor and the effective coupling coefficient have been introduced. For practical applications, both a sufficiently high coupling and as large as possible $Q$-values are the goal. However, there is a trade off between the two. By choosing a high acoustic impedance electrode the effective coupling coefficient of a BAW device can be significantly improved. 
Various loss mechanisms possible in BAW resonators are introduced and their association with the quality factor is discussed. The loss mechanisms are mainly acoustic and electric losses. The acoustic losses are again subdivided into losses such as acoustic leakage to the substrate and laterally escaping waves. The acoustic leakage through the reflector stack being the main theme of the thesis is further discussed in detail. The relation between the transmission of the acoustic waves in the reflector stack and the $Q$ factor is emphasized. The available methods for the experimental determination of the reflector stack performance have also been summarized.

The concept of acoustic dispersion in the context of BAW resonators has been discussed and the dispersion types have been introduced. Various aspects of the two types of dispersion curves have been reviewed. The concept of energy trapping and the origin of the spurious modes have been discussed. Due to the fact that a BAW resonator has finite dimensions, lateral standing waves occur in the active area of the resonator giving rise to spurious resonances. Few methods for suppressing spurious resonances have been addressed including the one by adopting a frame region.

\section{References}

[1] W. G. Cady, Piezoelectriciy, New York: McGraw Hill, 1946.

[2] H. P. Loebl, C. Metzmacher, R.F. Milsom, P. Lok, F. Van straten and A. Tuinhout, "RF bulk acoustic resonators and filters," Kluwer Journal of Electroceramics, vol.12, pp. 109-118, 2004.

[3] Y. Satoh, T. Nishihara, T. Yokoyama, M. Ueda and T. Miyashita, "Development of piezoelectric thin film resonator and its impact on future wireless communication systems," Japanese Journal of Applied Physics Part 1-Regular Papers Brief Communications $\mathcal{E}$ Review Papers, vol. 44, no.5A, pp. 2883-2894, 2005.

[4] B. A. Auld, Acoustic Fields and Waves in Solids, 1st ed. vol. 1: John Wiley \& Sons, Inc., 1973.

[5] K. M. Lakin, "Fundamental properties of thin film resonators," Proc. IEEE 45 ${ }^{\text {th }}$ Annual Symposium on Frequency Control, pp. 201-206, 1991.

[6] R. Aigner,"MEMS in RF filter applications: Thin-film bulk acoustic wave technology," Wiley Interscience: Sensors Update, vol. 12, pp. 175-210, 2003.

[7] J. F. Werner and A. J. Dyer, "The Relationship Between Plateback, Mass Loading and Electrode Dimensions for AT-Cut Quartz Crystals Having Rectangular Resonators Operating at Fundamental and Overtone Modes, " Proc. Annual Frequency Control Symposium pp. 40-53, 1976.

[8] K. M. Lakin, G. R. Kline, and K. T. McCarron, "Development of miniature filters for wireless applications," IEEE Transactions on Microwave Theory and Techniques, vol. 43, no.12, pp. 2933-2939, 1995.

[9] K. M. Lakin, "A review of thin-film resonator technology," IEEE Microwave Magazine, vol. 4, no. 4, pp. 61-67, 2003.

[10] R. Ruby," Review and comparison of Bulk Acoustic Wave FBAR, SMR technology," Proc. IEEE Ultrasonics Symposium, pp. 1029-1040, 2007.

[11]F .Z. Bi and B. P. Barber, "Bulk acoustic wave RF technology," IEEE Microwave magazine, 
vol. 9, no. 5, pp. 65-80, 2008.

[12]J. F. Rosenbaum, Bulk Acoustic Wave Theory and Devices, Artech House, 1988.

[13] K. Hashimoto, RF Bulk Acoustic Filters for communications, Artech House, 2009.

[14] N. Setter, D. Damjanovic, L. Eng, G. Fox, S. Gevorgian, S. Hong, A. Kingon, H. Kohlstedt, N. Y. Park, G. B. Stephenson, I. Stolitchnov, A. K. Taganstev, D. V. Taylor, T. Yamada, and S. Streiffer, "Ferroelectric thin films: Review of materials, properties, and applications," Journal of Applied Physics, vol. 100, pp. 051606-051606-46, 2006.

[15] R. Aigner, "Bringing BAW technology into volume production: The Ten Commandments and the seven deadly sins," IEEE Int. Symp. Acoust. Wave. Dev. for Future Mobile Communication Syst, 2007.

[16]J. S. Wang and K. M. Lakin, "Sputtered AlN films for bulk-acoustic-wave devices," Proc. IEEE Ultrasonics Symposium, pp. 502-505, 1981.

[17] K.M. Lakin, J.S. Wang, G.R. Kline, A.R. Landin, Y.Y. Chen, and J.D. Hunt, " Thin film resonators and filters, "Proc. IEEE Ultrasonics Symposium, pp. 466-475, 1982.

[18]W. P. Mason, Physical Acoustics Principles and Methods, vol. 1A, Academic Press, 1964.

[19]H. Jaffe and P. L. Smith, "IRE Standards on Piezoelectric Crystals - the Piezoelectric Vibrator - Definitions and Methods of Measurement, 1957," Proceedings of the Institute of Radio Engineers, vol. 45, pp. 353-358, 1957.

[20]J. D. Larson, P. Bradley, S. Wartenberg, and R. Ruby, "Modified Butterworth Van Dyke circuit for FBAR resonators and automated measurement system," Proc. IEEE Ultrasonics symposium, pp. 864-868, 2000.

[21]K. M. Lakin, "Modeling of thin film resonators and filters," in Proc. IEEE MTT-S International Microwave Symposium Digest, vol. 1, pp. 149-152, 1992.

[22] K. M. Lakin, G. R. Kline, and K. T. McCarron, "High-Q microwave acoustic resonators and filters," IEEE Transactions on Microwave Theory and Techniques, vol. 41, pp. 2139-2146, 1993.

[23] David. M. Pozar, Microwave Engineering, 3rd ed. John Wiley \&sons, Inc., Hoboken, New Jersey, 2007.

[24] S. Marksteiner, J. Kaitila, G. G. Fattinger and R. Aigner, “Optimization of acoustic mirrors for Solidly Mounted BAW resonators," Proc. IEEE Ultrasonics Symposium, pp. 329-332, 2005.

[25] T. Pensala, R. Thalhammer, J. Dekker, and J. Kaitila, "Experimental Investigation of Acoustic Substrate Losses in 1850-MHz Thin Film BAW Resonators", IEEE Trans. on Ultrasonics, Ferroelectrics and Frequency Control, vol. 56, no. 11, pp. 2544-2552, 2009.

[26] K. Nam, Y. Park, B. Ha, D. Shim, and I. Song, "Piezoelectric Properties of Aluminum Nitride for Thin Film Bulk Acoustic Wave Resonator, "Jrnl. Korean Phys. Soc., vol. 47, pp. s309-s312, 2005.

[27] G. G. Fattinger, "BAW resonators design considerations -An overview," Proc. IEEE International Frequency Control Symposium, pp. 762-767, 2008.

[28] J. Kaitila, "Review of wave propagation in BAW thin film devices progress and prospects," Proc. IEEE Ultrasonics Symposium, pp. 120-129, 2007.

[29] D. A. Berlincourt, D. R. Curran, and H. Jaffe, "Piezoelectric and piezomagnetic materials and their function as transducers," in Physical Acoustics, W. P. Mason, Ed. New York, Academic Press, 1964. 
[30]S. Chang, N. Rogacheva, and C. Chou, "Analysis of methods for determining electromechanical coupling coefficients of piezoelectric elements," IEEE Trans. on Ultrasonics, Ferroelectrics, and Frequency Control, vol. 42, no. 4, pp. 630-640, 1995.

[31]IEEE Standard on Piezoelectricity, ANSI/IEEE Std. 176-1987, 1988.

[32] K.M. Lakin, J. Belsick, J.F. McDonald, and K.T. McCarron. “Improved bulk wave resonator coupling coefficient for wide bandwidth filters," Proc. IEEE Ultrasonics Symposium, pp. 827-831, 2001.

[33]J. Olivares, E. Wegmann, M. Clement, J. Capilla, E. Iborra, and J. Sangrador, "Assessment of solidly mounted resonators with wide-band asymmetric acoustic reflectors," Proc. IEEE Ultrasonics Symposium, pp. 1677-1680, 2010.

[34] R. Aigner, "Volume manufacturing of BAW-filters in a CMOS fab," Proc. International Symposium on acoustic wave devices for future mobile communications systems, pp. 129134, 2004.

[35]J. W. Lobeek, R. Strijbos, A. B. M. Jansman, N. X. Li, A. B. Smolders and N. Pulsford, "High-Q BAW resonator on $\mathrm{Pt} / \mathrm{Ta}_{2} \mathrm{O}_{5} / \mathrm{SiO}_{2}$-based reflector stack," Proc. IEEE Microwave Symposium, pp. 2047-2050, 2007.

[36]R. Strijbos, A. B. M. Jansman, J. W. Lobeek, N. X. Li and N. Pulsford, “Design and characterization of high-Q Solidly-Mounted Bulk Acoustic Wave filters," Proc. IEEE Electronic components and technology conference, pp. 169-174, 2007.

[37]S. H. Lee, K. H. Yoon, and J. K. Lee, "Influence of electrode configurations on the quality factor and piezoelectric coupling constant of solidly mounted bulk acoustic wave resonators," Journal of Applied Physics, vol. 92, pp. 4062-4069, 2002.

[38] J. W. Lobeek and A. B. Smolders, "Design and industrialisation of solidly-mounted BAW filters," 2006 IEEE Mtt-S International Microwave Symposium Digest, Vols 1-5, pp. 386-389, 2006.

[39] S. Jose, A. B. M. Jansman, R. J. E. Hueting and J. Schmitz, “Optimized reflector stacks for solidly mounted bulk acoustic wave resonators," IEEE Trans. on Ultrasonics Ferroelectric and Frequency Control, vol. 57, no.12, pp. 2753-2763, 2010.

[40]R. Thalhammer and R. Aigner, "Energy loss mechanisms in SMR-type BAW devices," Proc. IEEE MTT-S International Microwave Symposium Digest, pp. 225-228, 2005.

[41]T. Pensala, Thin Film Bulk Acoustic Wave Devices. Performance Optimization and Modeling, Ph.D Dissertation, VTT Helsinki, Finland, 2011.

[42] R. Lanz, M. A. Dubois, and P. Muralt, "Solidly mounted BAW filters for the 6 to $8 \mathrm{GHz}$ range based on AlN thin films," Proc. Ultrasonics Symposium Proceedings, pp. 843-846, 2001.

[43] S. Marksteiner, G. G. Fattinger, R. Aigner and J. Kaitila, Acoustic Reflector for a BAW resonator providing specified reflection of both shear wave and longitudinal waves, US patent: 006933807B2., Aug. 2005.

[44] G. G. Fattinger, S. Marksteiner, J. Kaitila, and R. Aigner, “Optimization of acoustic dispersion for high performance thin film BAW resonators," Proc. IEEE Ultrasonics Symposium, pp. 1175-1178, 2005.

[45] K. Kokkonen, "Laser interferometers in physical acoustics," Proc. IEEE Ultrasonics Symposium, pp. 1036-1043, 2009.

[46] K. Kokkonen, T. Pensala, and M. Kaivola, "Interferometric Measurements of Dispersion Curves and Transmission Characteristics of the Acoustic Mirror in Thin Film BAW 
Resonator," in Proc. IEEE/MTT-S International Microwave Symposium, pp. 2071-2074, 2007.

[47]K. Kokkonen, T. Pensala, and M. Kaivola, "Dispersion and mirror transmission characteristics of bulk acoustic wave resonators," IEEE Transactions on Ultrasonics, Ferroelectrics, and Frequency Control, vol. 58, pp. 215-225, 2011.

[48] R. P. Feynman, R. B. Leighton, and M. Sands, The Feynman Lectures on Physics, Vol. 1: Mainly Mechanics, Radiation, and Heat: Addison Wesley, 2005.

[49] B. A. Auld, Acoustic Fields and Waves in Solids, 1st ed. vol. 2: John Wiley \& Sons, Inc., 1973.

[50]W. M. Ewing, Elastic Waves in Layered media, McGraw-Hill, 1957.

[51]M. J. S. Lowe, "Matrix techniques for modeling ultrasonic waves in multilayered," IEEE Transactions on Ultrasonics, Ferroelectrics, and Frequency Control, vol. 42, no. 4, pp. 525$542,1995$.

[52] H. Nowotny and E. Benes, "General one-dimensional treatment of the layered piezoelectric resonator with two electrodes," Jrnl of Acoust. Soc. Am., vol. 82, no.2, pp. 513-521, 1987.

[53] H. Lamb, "On Waves in an Elastic Plate," Proc. of Royal Society of London. Series A, vol. 93, pp. 114-128, 1917.

[54] A.B.M. Jansman, R. C. Strijbos, J. W. Lobeek, F. W. M Vanhelmont, J. J. M. Ruigrok, T. Pensala, M. Ylilammi, " Application of a Vibrating Membrane Model to Bulk-AcousticWave Resonators," in Proc. IEEE Ultrasonics Symposium, pp. 1045-1048, 2007.

[55]K. Kokkonen, T. Pensala, J. Meltaus, and M. Kaivola, " Direct measurement of spurious mode properties in thin film BAW resonator," Proc. IEEE Ultrasonics Symposium, pp. 420$423,2010$.

[56]T. Pensala, M. Ylilammi, J. Meltaus, K. Kokkonen, " Area and Dispersion Dependence of Vibration Shape and Coupling Coefficient in Thin Film BAW Resonators," in Proc. IEEE Ultrasonics Symposium, pp. 1661-1664, 2007.

[57] R. F. Milsom, H. P. Lobl, C. Metzmacher, P. Lok, A. Tuinhout, and F. van Straten, "2D model of solidly-mounted and membrane BAW devices," Proc. IEEE Ultrasonics Symposium, pp. 1802-1807, 2003.

[58]W. Shockley, D. R. Curran, and D. J. Koneval, "Energy trapping and related studies of multiple electrode filter crystals", Proc. 17th Annual Frequency Control Symposium, pp. 88-126, 1963.

[59]P. Schnabel, "Dispersion of thickness vibrations of piezoelectric disk resonators," IEEE Transactions on Sonics and Ultrasonics, vol. 25, no.1, pp. 16-24, 1978.

[60] R. D. Mindlin and M. A. Medick, "Extensional Vibrations of Elastic Plates," J. Appl. Mech. Trans. ASME, 26, 561, 1959.

[61] F. Thalmayr, K. Hashimoto, T. Omori, and M. Yamaguchi, "Plate mode propagation losses in solidly mounted resonators," IEEE Transactions on Ultrasonics, Ferroelectrics, and Frequency Control, vol. 57, pp. 2844-2849, 2010.

[62] S. Jose, R. J. E. Hueting and A. B. M. Jansman, "Acoustic dispersion of Solidly Mounted Resonators with an optimized reflector stack for dual wave reflection," Proc. IEEE Ultrasonics Symposium, September, pp. 291-294, 2010.

[63]J. Kaitila, M. Ylilammi, J. Ella, and R. Aigner, "Spurious resonance free bulk acoustic wave resonators," in Proc. IEEE Ultrasonics Symposium, pp. 84-87, 2003. 
[64] R. C. Ruby, J. D. Larson, R. S. Fazzio, and C. Feng, "Performance degradation effects in FBAR filters and resonators due to Lamb Wave Modes," 2005 IEEE Ultrasonics Symposium, pp. 1832-1835, 2005.

[65] A. Link, E. Schmidhammer, H. Heinze, M. Mayer, B. Bader, and R. Weigel, “Appropriate Methods to Suppress Spurious FBAR Modes in Volume Production," IEEE MTT-S Microwave Symposium Digest, pp. 394-397, 2006.

[66] R. Thalhammer, J. Kaitila, S. Zieglmeier and L. Elbrecht, "Spurious mode suppression in BAW resonators," Proc. IEEE Ultrasonics Symposium, pp. 456-459, 2006.

[67]S. Jose, R. J. E. Hueting and A. B. M. Jansman, "On the rule of thumb for flipping of the dispersion relation in BAW devices," Proc. IEEE Ultrasonics Symposium, October, 2011. 

In this chapter, we propose two novel approaches for the reflector stack design that effectively reflects both longitudinal and shear waves. The design approaches have been derived from optics; the first one employs the stop-band theory and the second one takes advantage of the periodic nature of reflection spectra in a Bragg reflector, the diffraction grating design approach. It is demonstrated using FEM simulations that the design schemes are applicable for various material combinations. 
The quality factor $(Q)$ of traditional Solidly Mounted Bulk Acoustic Wave Resonators (SMRs) is traditionally limited by substrate losses, because the conventional quarter wave reflector stack reflects only the longitudinal acoustic waves and not the shear waves. In order to obtain high- $Q$ SMRs, the reflector stack should effectively reflect both the waves. Modern reflector stacks do reflect both waves, but design rules for such reflectors have not yet been reported. In this chapter, we propose two different design approaches derived from optics to tailor the acoustic reflector for effective reflection of both longitudinal and shear waves. The first one employs the stop-band theory in optics; the second one takes advantage of the periodic nature of reflection spectra in a Bragg reflector, the diffraction grating design approach. Analytical models are presented to validate the approaches. In addition, Finite element modeling (FEM) tools have been used to verify the results. Section 3.1 presents a background review of the reflector stack design in SMRs. The stop-band theory based approaches are discussed in section 3.2 and the diffracting grating based approaches are discussed in section 3.3. 2D FEM simulations for verifying the approaches are presented in section 3.4. Section 3.5 overviews a comparison of the approaches. Finally, section 3.6 summarizes the chapter.

\subsection{Background}

The design of the reflector stack is critical in the performance of high $Q$ SMRs [1] - [7]. Figure 3.1 shows a typical SMR structure in which the piezoelectric resonator is isolated from the substrate using an acoustic reflector stack (or mirror) [7]. The concept of acoustic mirrors for thin film resonators was first reported by Newell in 1965 [8]. The SMART structure was indeed "smart" in reflecting the longitudinal acoustic waves. In mid-1990s, Lakin et al. [7], [9] realized the SMRs. These SMRs employ a quarter-wave length reflector stack which was optimized to reflect longitudinal waves only. The best reflection is obtained if the mirror or reflector stack consists of alternating layers of low and high acoustic impedances, and that the ratio of these impedances is maximal. For optimal reflection, the layer thickness $t_{\mathrm{n}}$ of each layer $\mathrm{n}$ is a quarter of the wavelength for longitudinal waves $\lambda_{\text {long }}$ at resonance frequency $f_{\mathrm{R}}[10]$ :

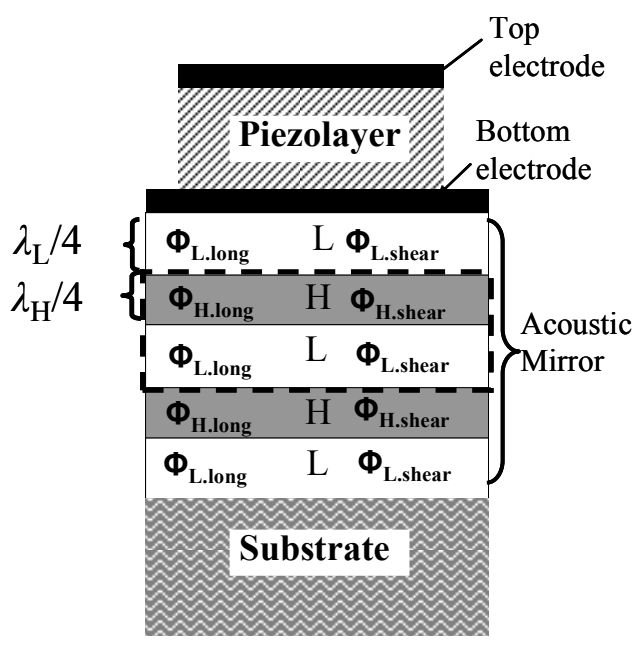

Figure 3.1: 


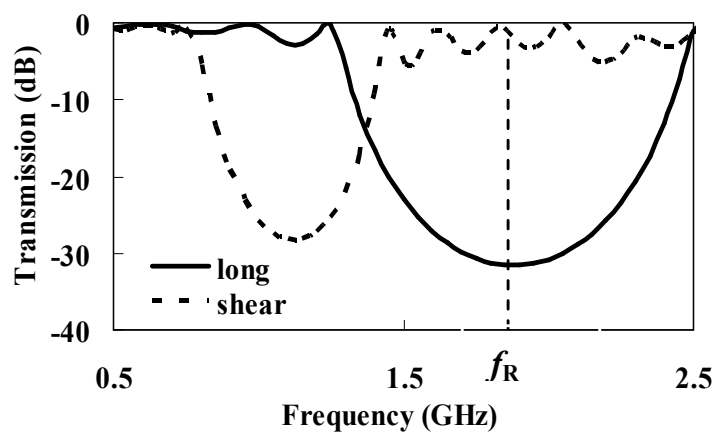

Figure 3.2: $\quad$ Calculated transmission curves of longitudinal and shear waves of a nine layer $\mathrm{SiO}_{2} / \mathrm{Ta}_{2} \mathrm{O}_{5}$ quarter-wave reflector stack optimized for minimum transmission for longitudinal waves at the resonance frequency $f_{R}=1.88 \mathrm{GHz}$. Longitudinal transmission at $f_{R}$ is $-31.5 \mathrm{~dB}$ and shear transmission at $f_{R}$ is $-0.59 \mathrm{~dB}$ at resonance frequency.

$t_{n}=\frac{\lambda_{\text {long }}}{4}$

However, in a laterally finite resonator, the wave is never a pure bulk wave but a plate wave with contribution from both longitudinal and shear wave modes [11]. This leads to shear wave generation at the lateral edges of the resonator. These shear waves would leak into the substrate and cause acoustic losses in the resonator, if the reflector stack does not provide a shear reflection. In the quarter-wave reflector stack designed using eq.(3.1) shear waves are not optimally reflected. This can be explained by the fact that the shear wave velocity is typically about half of the longitudinal wave velocity [3].

By implementing a transmission line model (see section 2.2.1) and with the approach of reflection [1] but with the top-most layer of the reflector stack as the reference point, the transmission curve as a function of frequency is computed with MathCAD [12]. For a multilayer reflector stack, the effective load impedance can be obtained by successive use of the transmission line equation (eq.(2.6)). In the case of quarter wavelength layers, the phase across each layer $\theta=\pi / 2$ and hence eq.(2.6) reduces to:

$$
Z_{\text {in }}=\frac{Z_{s}^{2}}{Z_{l}} \text {. }
$$

By numbering sections from top of the reflector (for e.g. nine layer reflector stack), the effective impedance of the reflector stack is then given by:

$$
Z_{\mathrm{RS}}=\frac{Z_{9}{ }^{2} \cdot Z_{7}{ }^{2} \cdot Z_{5}{ }^{2} \cdot Z_{3}{ }^{2} \cdot Z_{1}{ }^{2}}{Z_{8}{ }^{2} \cdot Z_{6}{ }^{2} \cdot Z_{4}{ }^{2} \cdot Z_{2}{ }^{2} \cdot Z_{S S}}
$$

where $Z_{1}, Z_{3} \ldots Z_{9}$ are the acoustic impedances of the low impedance layers, $Z_{2}, Z_{4} \ldots Z_{8}$ are the acoustic impedances of the high impedance layers and $Z_{S S}$ that of the substrate. 
Note that in this special case of quarter wavelength, the lower boundary condition for the piezoelectric layer is zero stress, just as if it had an interface with air. In the case of half wavelength layers, $\theta=\pi$ and hence $Z_{\mathrm{RS}}=Z_{\mathrm{SS}}$ making the mirror ineffective.

Figure 3.2 shows the resulting transmission curves of a nine layer $\mathrm{SiO}_{2} / \mathrm{Ta}_{2} \mathrm{O}_{5}$ $(762 \mathrm{~nm} / 588 \mathrm{~nm})$ quarter-wave stack optimized at $f_{\mathrm{R}}=1.88 \mathrm{GHz}$ for minimum transmission of longitudinal waves. It can be seen that the minimum transmission of longitudinal waves corresponds to maximum transmission for shear waves. This emphasizes that dual acoustic wave reflection in this particular stack is not possible.

The necessity for reflection of both types of waves for high $Q$ resonators was recognized earlier and the influence of shear waves on the $Q$ factor is reviewed in [13]. A possible solution to obtain dual wave reflection would be employing two sets of stacks; one reflecting longitudinal and another set reflecting shear waves. However, this would increase the number of layers and hence the fabrication complexity. In sections 3.2 and 3.3, we present several design approaches derived from optics to solve this paradox.

\subsection{Stop-band theory based approaches}

Two design approaches [6], [14] are discussed in this section. Section 3.2.1 describes an approach to modify the stacks to effectively reflect both longitudinal and shear waves, based on the stop-band theory [15] in optics. A more general optimization scheme, the phase error approach, based on the same theory is presented in section 3.2.2. Note that the translation from optics towards acoustics is an important issue. The optical reflector stack consists of alternating layers of high and low refractive indices whereas an acoustical reflector stack consists of high and low acoustic impedances. For the BAW filters we need to reflect longitudinal and shear acoustic waves at the same single $f_{\mathrm{R}}$ but each having a different wave velocity, whereas in optical filters light with a fixed velocity is filtered at different wavelengths.

\subsubsection{The basic stop-band theory approach}

It was as early as in 1917 that Lord Rayleigh [16] studied the natural stratification in some insects which are periodic structures of alternative high and low optical refractive indices. It was perhaps here the first stop-band theory was defined. It was later redeveloped by various authors to study optics in thin film dielectric periodic structures [15]. Presently the theory is well defined in optics of thin-films.

For the simplest periodic structure, the basic unit consists of two dielectric layers as shown in Figure 3.1 (dashed lines) with high $(\mathrm{H})$ and low $(\mathrm{L})$ refractive indices. Optics of thin films defines a stop-band being a high reflection band or equivalently a low transmission band which is a characteristic feature of any periodic structure. The cardinal point of the stop-band is defined as the point of maximum reflection (minimum transmission) in this region. The well-known quarter wavelength stack (denoted as LH) gives reflection for the frequencies at which the phase drops over the high and low index layers are both $\pi / 2$. One can deviate from these equal phases and still obtain reflection bands. 
The stop-band theory [15] states that an L(c.H)-stack (i.e. a stack consisting of bi-layers where the ratio of phases over the low and high index equals an integer $c$ ) gives a maximum reflection for the frequencies at which the phase drop over one of the layers ( $\mathrm{L}$ or $\mathrm{H}$ ) equal of the so-called cardinal points (transmission minima) $\varphi_{\mathrm{n}}$ :

$\varphi_{\mathrm{n}}=\frac{n \pi}{1+c}$

where $n$ takes integer values $n \leq c$ with the detuning parameter $c=\phi_{H} / \phi_{L} \cdot \phi_{L}$ and $\phi_{H}$ are the phase drops in low index and high index layers respectively. If we choose $\varphi_{\mathrm{n}}$ as the phase of the low-index layer, the corresponding phase of the high index layer is $c \cdot \varphi_{\mathrm{n}}$. Note that for $c=1$ the stack reduces to the quarter-wavelength (LH) case.

Strictly speaking the stop band theory restricts $c$ to be integer numbers. However, one can choose $c$ to be non-integer at the expense of non-equal reflection efficiency. Still, the reflection bands appear at frequencies for which the phase over the L-layer equals $n \pi /$ $(1+c)$. Furthermore, large values of $n$ and $c$ lead to large phase drops over the layers leading to relatively thick layers. For fabrication cost reasons this is undesired. Therefore the numbers $c$ and $n$ are kept at a minimum.

Further, for $\phi_{L}$ and $\phi_{H}$ at maximum reflection also holds:

$\phi_{\mathrm{L}}+\phi_{\mathrm{H}}=n \pi$.

Hence, eqns. (3.4) and (3.5) state that [15]:

$\phi_{L}+\phi_{H}=(1+c) \varphi_{\mathrm{n}}=n \pi$.

Focusing now on an acoustical reflector, the objective is to design a reflector stack that reflects both longitudinal and shear waves. The stop band theory has given us a degree of freedom - the detuning parameter $c$. Hence the task is to find thicknesses of the low and high index layer, $t_{\mathrm{L}}$ and $t_{\mathrm{H}}$ respectively, such that the sum of phases drops over a bilayer equals a multiple of $\pi$ for both types of waves.

Let us focus on the longitudinal acoustic wave first. Consider an $\mathrm{L}(c \cdot \mathrm{H})$ stack, where $c$ is now defined in terms of longitudinal waves:

$$
c=\frac{\phi_{\mathrm{H} . \mathrm{long}}}{\phi_{\mathrm{L} . \mathrm{long}}} .
$$

$\mathrm{L}$ and $\mathrm{H}$ now denote the layers of low and high acoustic impedance respectively. 
At the frequency of maximum reflection, the cardinal points equal the phase drops over the layers $\left(\varphi_{\mathrm{n} . \mathrm{L}}=\phi_{\mathrm{L}}, \varphi_{\mathrm{n} . \mathrm{H}}=\phi_{\mathrm{H}}\right)$ and let's say the longitudinal waves will have reflection bands at the frequency for which the phase drop over the L-layer, $\phi_{L . l o n g}$ is:

$\phi_{\mathrm{L} . \text { long }}=\frac{n \pi}{1+c}$,

From eqns.(3.7) and (3.8), we can find the phase drop over the H-layer as:

$\phi_{\mathrm{H} . \text { long }}=\frac{n c \pi}{1+c}$.

Setting this frequency equal to a desired frequency $f_{R}$ at which a good reflection is desired, we can calculate the thickness of the H-layer and L-layer as:

$t_{\mathrm{L}}=\frac{\phi_{\mathrm{L} . \text { long }}}{2 \pi} \cdot \frac{v_{\mathrm{L} . \text { long }}}{f_{\mathrm{R}}}=\frac{n}{2(1+c)} \lambda_{\mathrm{L} . \text { long }}$,

$t_{\mathrm{H}}=\frac{\phi_{\mathrm{H} . \text { long }}}{2 \pi} \cdot \frac{v_{\mathrm{H} . \text { long }}}{f_{\mathrm{R}}}=\frac{c n}{2(1+c)} \lambda_{\mathrm{H} . \text { long }}$,

where $v_{\text {L.long }}$ and $v_{\text {H.long }}$ are the velocities of the longitudinal waves in low and high acoustic impedance layer respectively and $\lambda_{\text {L.long }}$ and $\lambda_{\text {H.long }}$ are the corresponding wavelengths.

The phase drop over these layers for shear waves equals:

$\phi_{\text {L.shear }}=2 \pi f_{R} \cdot\left(\frac{t_{L}}{v_{\text {L.shear }}}\right)$.

Substituting $t_{\mathrm{L}}$ from eq. (3.10) and simplifying:

$\phi_{\text {L.shear }}=\frac{n \pi}{(1+c)} \cdot\left(\frac{v_{\text {L.long }}}{v_{\text {L.shear }}}\right)=\frac{n \pi}{(1+c)} \cdot K_{\mathrm{L}}$.

Similarly we can find the phase drop of the shear waves in the L layer as:

$\phi_{\mathrm{H} \text {.shear }}=\frac{n \cdot c \pi}{(1+c)} \cdot\left(\frac{v_{H . \text { long }}}{v_{\text {H.shear }}}\right)=\frac{n \cdot c \pi}{(1+c)} \cdot K_{\mathrm{H}}$,

where $v_{\text {L.shear }}$ and $v_{\text {H.shear }}$ are the velocity of shear waves in low and high impedance layers respectively and $K_{\mathrm{L}}=v_{\text {L.long }} / v_{\text {L.shear, }} K_{\mathrm{H}}=v_{\text {H.long }} / v_{\text {H.shear }}$. 


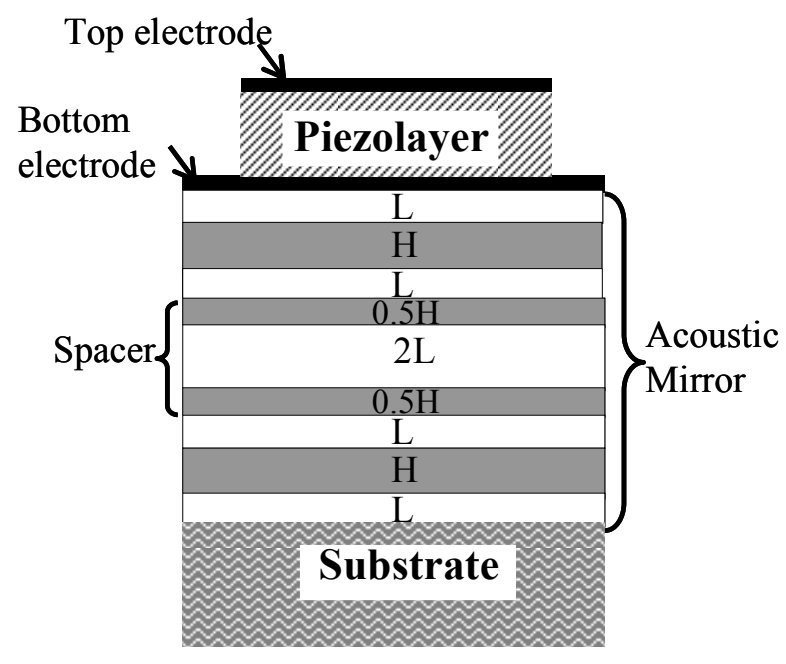

Figure 3.3: $\quad$ Improved layer configuration with the optimized thicknesses for the acoustic mirror of a Solidly Mounted BAW Resonator (SMR). The spacer layer is according to [17].

The sum of the phases of shear waves in L and H layers (see eqns. (3.12) and (3.13)) will result in $n \pi$. For most of the practical materials the ratios $K_{\mathrm{L}}$ and $K_{\mathrm{H}}$ are not far from 2. Hence if the sum of phase drops for the longitudinal wave is $n \pi$ then the sum of phase drops for the shear waves will be close to $2 n \pi$, which also follows eq. (3.5).

With this approximation, we can calculate the thicknesses of $\mathrm{L}$ and $\mathrm{H}$ layer to reflect both the waves. Acknowledging that the longitudinal wave is the primary wave in a BAW resonator carrying most acoustic energy we emphasize our optimization to this type of wave. So we set the detuning parameter $c$ for longitudinal waves equal to an integer number. The quarter wave stack $(c=1, n=1$ for longitudinal waves) gives $c=1$, and effectively $n=2$ for shear waves if the ratios $K_{\mathrm{L}}$ and $K_{\mathrm{H}}$ equal 2 . This is not a reflection band, since $\mathrm{n}$ should satisfy the condition $n \leq c$. Next in row is $c=2, n=1$ for the longitudinal wave. This gives $c=2, n=2$ for the shear wave, fulfilling all the conditions of a cardinal point. To conclude the L2H configuration can simultaneously reflect both longitudinal and shear waves.

The thicknesses given by eq. (3.10) provide maximum reflection for both longitudinal and shear waves at a particular frequency $1.88 \mathrm{GHz}$. This elementary method often leads to satisfactory results if $K_{\mathrm{L}}$ and $K_{\mathrm{H}}$ is not too far from 2 for both materials. Also for cases where the average of $K_{\mathrm{L}}$ and $K_{\mathrm{H}}$ is around 2 reasonable reflection coefficients can be expected as the derivation is based on the $\mathrm{L} 2 \mathrm{H}$ configuration. It is inferred from the simulations that for optimum results for dual reflection of longitudinal and shear waves in low and high impedance layers, $c$ can be taken as the average of velocity ratios as:

$c=\frac{K_{L}+K_{H}}{2} \approx 2$

The optimized thicknesses obtained using eq.(3.10) can be used in an improved layer configuration by introducing a spacer layer $2 \mathrm{H} 2 \mathrm{~L} 2 \mathrm{H} \mathrm{[17]} \mathrm{to} \mathrm{further} \mathrm{optimize} \mathrm{the}$ 


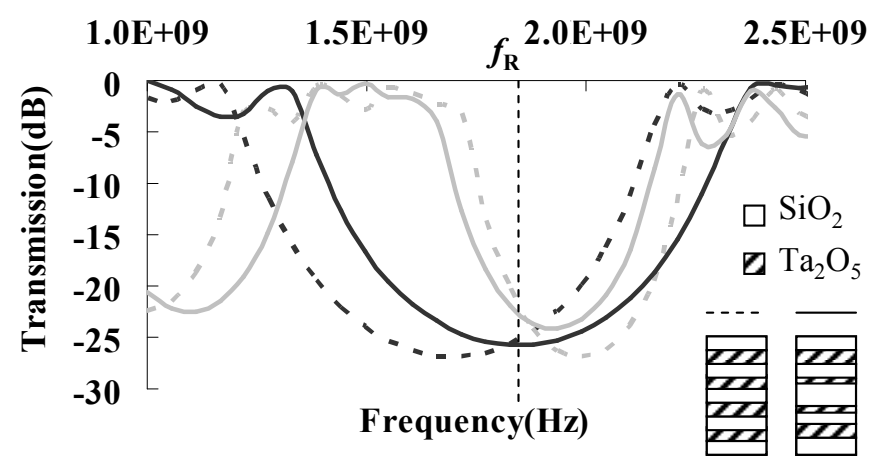

Figure 3.4: $\quad$ Transmission curves of a nine layer $\mathrm{SiO}_{2} / \mathrm{Ta}_{2} \mathrm{O}_{5}$ optimized reflector stack based on the stop-band theory approach with and without the spacer layer. Black lines indicate longitudinal and grey indicate shear waves. Solid lines represent the configuration with the spacer layer and dashed lines represent the ones without the spacer layer. Longitudinal transmission is $-25.7 \mathrm{~dB}$ and shear transmission is $-22.8 d B$ at resonance frequency.

reflection bands for both types of waves. In optics it was suggested to tune this spacer layer by using a trial and error method for aligning the edges of the bands and hence improving the performance [18]. However, we optimized the reflector stack, which is actually a Fabry-Perot resonator, using a transmission line model [19]. We observed that a $0.5 \mathrm{H} 2 \mathrm{~L} 0.5 \mathrm{H}$ configuration acts as a Fabry-Perot cavity for both longitudinal and shear waves. The improved layer configuration of the acoustic mirror is (LHL) $(0.5 \mathrm{H} 2 \mathrm{~L} 0.5 \mathrm{H})$ (LHL) which is shown in Figure 3.3. Note that the figures in the configuration indicate the relative layer thickness, where the thicknesses of $\mathrm{L}$ and $\mathrm{H}$ layers are obtained from eq.(3.10).

The one-dimensional analytical model results are presented in Figure 3.4. It shows the transmission curve for the optimized reflector stack with and without the spacer. It can be seen from the figure that using the optimized thicknesses calculated by the new design approach has minimized the transmission at $1.88 \mathrm{GHz}$, and using these

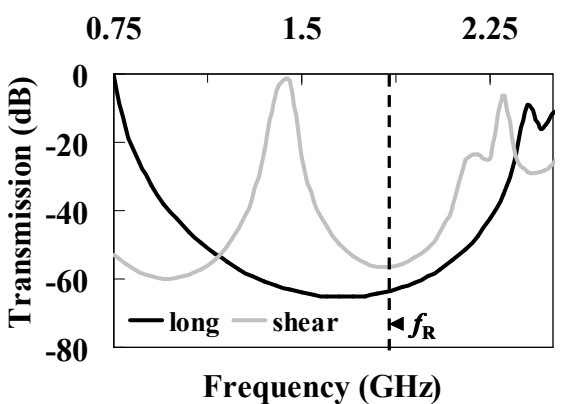

(a)

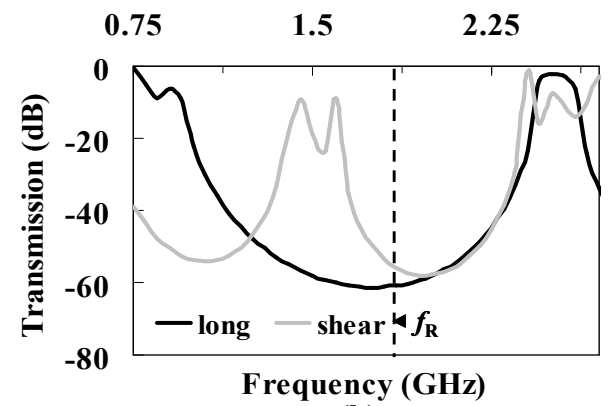

(b)

Figure 3.5: Transmission curves of a nine layer optimized reflector stack of (a) $\mathrm{SiO}_{2} / \mathrm{W}$ and (b) $\mathrm{SiOC} / \mathrm{Ta}_{2} \mathrm{O}_{5}$ obtained using stop-band theory approach. In both the cases, the transmission of longitudinal and shear waves are well below $-55 \mathrm{~dB}$ at resonant frequency. 
thicknesses in the (improved) spacer configuration brings the transmission curves into coincidence.

To demonstrate the usefulness of the approach, the new design approach was applied for different material combinations such as $\mathrm{SiO}_{2} / \mathrm{W}, \mathrm{SiOC} / \mathrm{Ta}_{2} \mathrm{O}_{5}, \mathrm{SiOC} / \mathrm{W}, \mathrm{SiOC} / \mathrm{Pt}$ and $\mathrm{SiOC} / \mathrm{TiN}$. In all the cases, the optimized layer stacks in the new configuration show efficient reflection of both longitudinal and shear waves. Figure 3.5 shows the curves demonstrated for different material combinations. The much lower transmission coefficients compared to Figure 3.5 are related to the use of high impedance contrast $(1: 12)$ materials in the reflector stack.

Analogue to an earlier report [20], the dependence of the layer thickness on the mirror transmission has been investigated. The layer thickness was varied individually per ten percentage thickness. Transmission of longitudinal and shear waves with the thickness variations are shown in Table 3.1. The transmission for longitudinal waves appears to be most sensitive to the thickness of the first $\mathrm{Ta}_{2} \mathrm{O}_{5}$ layer and least sensitive to that of the first TEOS layer. In the case of shear waves, it does not follow a rigid trend, although in many cases shear transmission is mainly sensitive to the thickness of the middle spacer layer. This is explained by 2D FEM simulations discussed in section 3.4. An increase in 10 percentage thickness only can bring a change of $\pm 1 \mathrm{~dB}$.

\subsubsection{The phase error approach}

The basic reflector design method gives best results if $K_{\mathrm{L}}$ and $K_{\mathrm{H}}$ are close to 2 which is true for most of the commonly applied materials [3]. However in some cases in which the velocity ratio deviates much from 2 , the basic stop-band design approach becomes less accurate. In this section, we propose a more general approach for dual reflection by minimizing the so-called phase error. This phase error (or phase offset) is the phase difference between the phase at the cardinal points and the calculated phase. The thicknesses are optimized in such a way that the phase offset from the transmission minima (cardinal points) for longitudinal and shear waves are equal and consequently

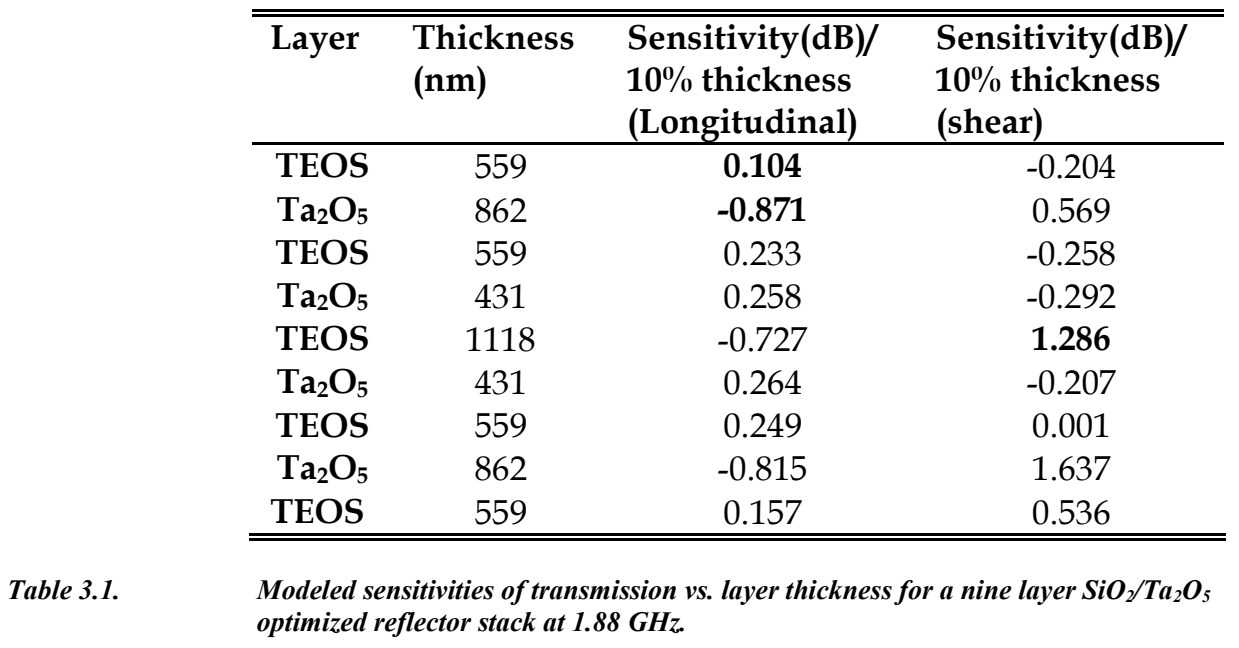


the phase error is minimal in both the cases. Defining $\beta_{\mathrm{L}}$ and $\beta_{\mathrm{H}}$ as the wave numbers in low and high impedance layers respectively, the phase drops $\phi_{\mathrm{L}}=\beta_{\mathrm{L}} \cdot t_{\mathrm{L}}$ and $\phi_{\mathrm{H}}=\beta_{\mathrm{H}} \cdot t_{\mathrm{H}}$ can be conveniently written in terms of thicknesses and wave velocity as (refer to eq. (3.5)):

$\phi_{\mathrm{L}}+\phi_{\mathrm{H}}=\frac{2 \pi t_{\mathrm{L}}}{\lambda_{\mathrm{L}}}+\frac{2 \pi t_{\mathrm{H}}}{\lambda_{\mathrm{H}}}=2 \pi f_{\mathrm{R}}\left(\frac{t_{\mathrm{L}}}{v_{\mathrm{L}}}+\frac{t_{\mathrm{H}}}{v_{\mathrm{H}}}\right)=n \pi$.

In acoustics, $v_{\mathrm{L}}$ and $v_{\mathrm{H}}$ have a different value for the case of longitudinal and shear waves. Therefore, we need to obtain thickness $t_{\mathrm{L}}$ and $t_{\mathrm{H}}$, such that eq. (3.15) is fulfilled for both longitudinal and shear waves at one frequency $f_{\mathrm{R}}$ :

and

$$
\begin{aligned}
& 2 \pi f_{\mathrm{R}}\left(\frac{t_{\mathrm{L}}}{v_{\mathrm{L} . \text { long }}}+\frac{t_{\mathrm{H}}}{v_{\mathrm{H} . \text { long }}}\right)=n_{1} \pi, \\
& 2 \pi f_{\mathrm{R}}\left(\frac{t_{\mathrm{L}}}{v_{\mathrm{L} . \text { shear }}}+\frac{t_{\mathrm{H}}}{v_{\mathrm{H} \text {.shear }}}\right)=n_{2} \pi .
\end{aligned}
$$

This set of two equations with two unknowns $t_{\mathrm{H}}$ and $t_{\mathrm{L}}$ is solvable for all values of positive integers $n_{1}$ and $n_{2}$. Again, the ratio of longitudinal and shear wave velocities of most materials is around 2. For such values, only non-negative values for the thicknesses are found if $n_{1}$ and/or $n_{2}$ are larger than 3. This translates into layer thicknesses well above $1000 \mathrm{~nm}$ for the frequency range of 1-3 GHz. Thicker layers increase the fabrication complexities.

Let us allow a phase error $\Delta \phi$ in eq. (3.16) such that it has the same value for shear and longitudinal waves. In order to keep the thicknesses of the layers small, we set $n_{1}$ and $n_{2}$ to 1 and 2, respectively, like in a L2H configuration. Hence,

$\phi_{\mathrm{L} . \text { long }}+\phi_{\mathrm{H} . \text { long }}=\pi+\Delta \phi$,

and

$\phi_{\mathrm{L} \text {.shear }}+\phi_{\mathrm{H} . \text { shear }}=2 \pi-\Delta \phi$.

$\phi_{\mathrm{L} \text {.long }}$ and $\phi_{\mathrm{L} \text {.shear }}$ are the phase drops over the low-impedance layer for the longitudinal and shear waves at the same frequency $f_{\mathrm{R}}$. Likewise, $\phi_{\mathrm{H} \text {.long }}$ and $\phi_{\mathrm{H} \text {.shear }}$ are the phase drops over the high-impedance layer at this frequency.

Introducing $K_{\mathrm{L}}$ and $K_{\mathrm{H}}$ in eq. (3.18) gives:

$K_{\mathrm{L}} \phi_{\text {L.long }}+K_{\mathrm{H}} \phi_{\mathrm{H} . \text { long }}=2 \pi-\Delta \phi$.

By summing eq. (3.17) and eq. (3.19), the phase difference drops out: 


\begin{tabular}{cc}
\hline \hline Materials & $K=v_{\text {long }} / v_{\text {shear }}$ \\
\hline $\mathrm{SiO}_{2}$ & 1.64 \\
$\mathrm{Ta}_{2} \mathrm{O}_{5}$ & 1.81 \\
$\mathrm{~W}$ & 1.77 \\
$\mathrm{SiOC}$ & 1.55 \\
$\mathrm{AlN}$ & 1.85 \\
$\mathrm{Pt}$ & 2.43 \\
\hline \hline
\end{tabular}

Table 3.2:

Summary of velocity ratio used for reflectors. Data obtained from [23].

$\phi_{\text {L.long }}\left(1+K_{\mathrm{L}}\right)+\phi_{\text {H.long }}\left(1+K_{\mathrm{H}}\right)=3 \pi$.

Now introducing $c$ in eq. (3.20) gives:

$\phi_{\text {L.long }}\left(1+c+K_{\mathrm{L}}+c K_{\mathrm{H}}\right)=3 \pi$.

This results in:

$$
\begin{aligned}
& \phi_{\mathrm{L} . \text { long }}=\frac{3 \pi}{1+c+K_{\mathrm{L}}+c K_{\mathrm{H}}}, \\
& \phi_{\mathrm{H} \text {.long }}=c \phi_{\mathrm{L} . \text { long }}=\frac{3 \pi c}{1+c+K_{\mathrm{L}}+c K_{\mathrm{H}}} .
\end{aligned}
$$

Then, the associated layer thicknesses are calculated in a straight- forward way:

$$
\begin{aligned}
& t_{\mathrm{L}}=\frac{\phi_{\mathrm{L} . \text { long }}}{2 \pi} \lambda_{\mathrm{L} . \text { long }}=\frac{3 / 2}{1+c+K_{\mathrm{L}}+c K_{\mathrm{H}}} \lambda_{\mathrm{L} . \text { long }}, \\
& t_{\mathrm{H}}=\frac{\phi_{\mathrm{H} . \text { long }}}{2 \pi} \lambda_{\mathrm{H} . \text { long }}=\frac{3 c / 2}{1+c+K_{\mathrm{L}}+c K_{\mathrm{H}}} \lambda_{\mathrm{H} . \text { long }} .
\end{aligned}
$$

We have come to an expression for the layer thicknesses giving an equal phase error $\Delta \phi$ in eqs. (3.17) and (3.18). The coefficient $c$ is a degree of freedom that we will use later. Substituting eq. (3.22) in eq. (3.17) gives the phase error applicable for both longitudinal and shear waves:

$$
\Delta \phi=\frac{\left(2+2 c-K_{\mathrm{L}}-c K_{\mathrm{H}}\right) \pi}{1+c+K_{\mathrm{L}}+c K_{\mathrm{H}}} .
$$

In summary, a reflector stack can be optimized according to eq. (3.23). In this case $c$ is a variable parameter. We have verified the performance of reflectors, built up according to eq. (3.23) for various reflector materials the properties of which are given in Table 3.2. In Figure 3.6, we present the transmission against $c$ calculated for reflector stacks with varying number of layers using different material combinations, demonstrating that good performance is obtained for $1<c<2$. The figure shows that for a given material combination, in each bi-layer, the transmission decreases by a fixed amount and this 


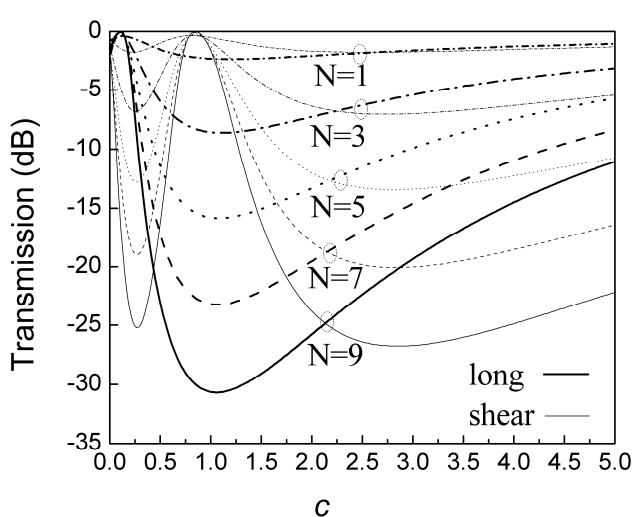

(a) $\mathrm{SiO}_{2} / \mathrm{Ta}_{2} \mathrm{O}_{5}$

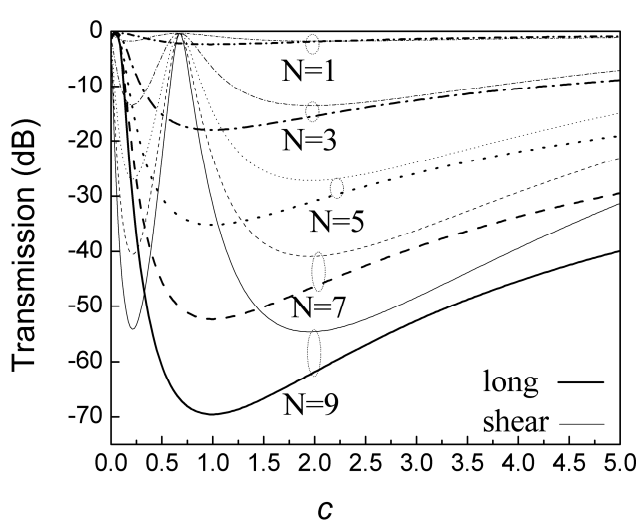

(c) $\mathrm{SiO}_{2} / \mathrm{Pt}$

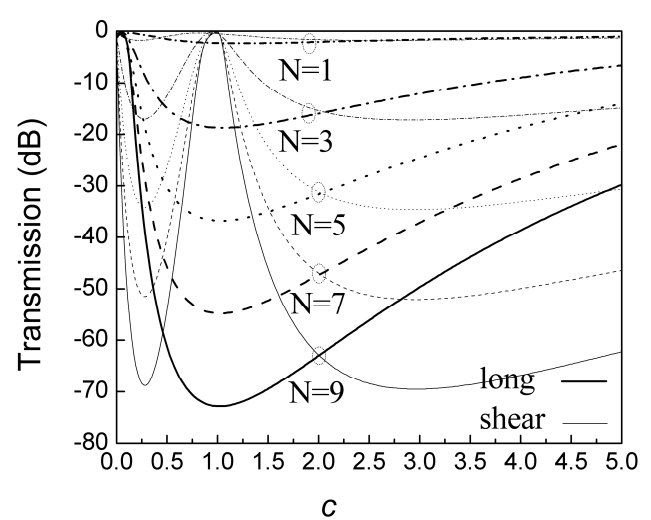

(b) $\mathrm{SiO}_{2} / \mathrm{W}$

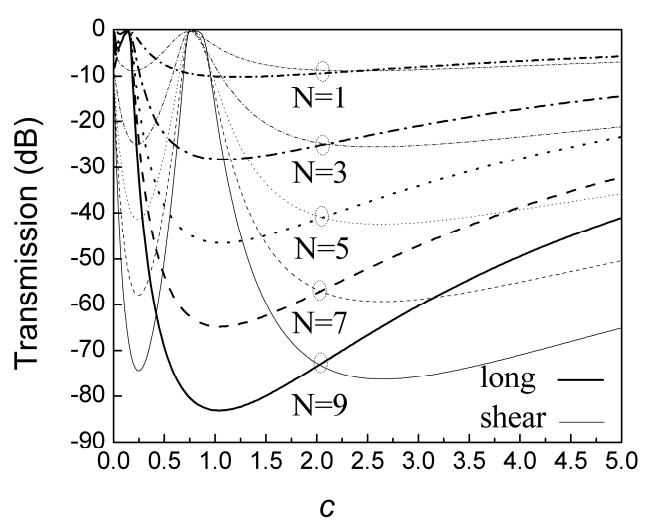

(d) $\mathrm{SiOC} / \mathrm{AIN}$

Figure 3.6:

Transmission vs. the phase ratio $c$ at resonant frequency for reflector stacks with varying number of layers demonstrated for different material combinations (a) $\mathrm{SiO}_{2} / \mathrm{Ta}_{2} \mathrm{O}_{5}$ (b) $\mathrm{SiO}_{2} / W$ (c) $\mathrm{SiO}_{2} / \mathrm{Pt}$ (d) SiOC/AlN. In the calculations the mirror stack was loaded with AlN on the top and Si at the bottom. $N$ represents the number of layers used for the mirror stack.

decreases more when there are more reflector layers. A low transmission for longitudinal and shear waves is also obtained for $c$ values of around 0.5. Nonetheless, this working point is less attractive; for most material combinations the transmission for longitudinal waves is larger than for shear waves. For a high-performance BAW resonator, the transmission of longitudinal waves should be lower than $-35 \mathrm{~dB}$, for shear waves it should be lower than $-18 \mathrm{~dB}$ [2].

In Figure 3.7, we present the transmission curves for nine layer reflector stacks with thicknesses optimized for a minimum dual wave transmission at resonance frequency using eq. (3.23) by varying $c$. The advantage of $c$ being a tunable parameter is that the transmission of longitudinal and shear waves can be improved according to the user requirements and hence this method allows the designer to find the trade-off between longitudinal and shear reflection by choosing the proper value for $c$. The thicknesses obtained with this design approach can also be combined with the spacer layer configuration proposed earlier [14] (see Figure 3.3) for further bringing the bands into coincidence and hence achieving the dual wave reflection in the same frequency band. 


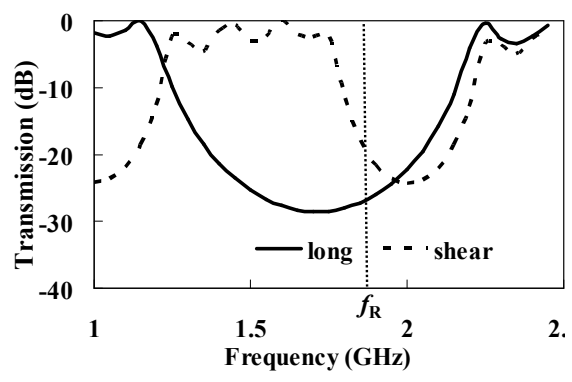

(a) $\mathrm{SiO}_{2} / \mathrm{Ta}_{2} \mathrm{O}_{5}$

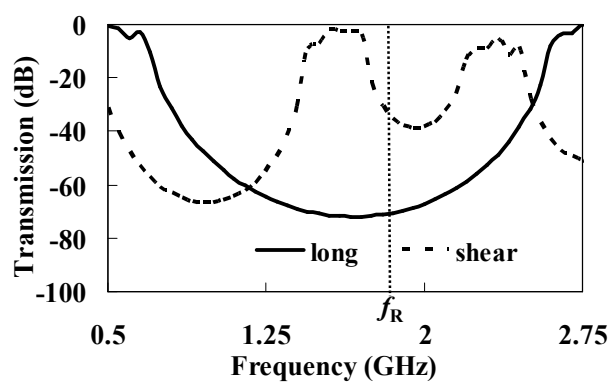

(b) $\mathrm{SiO}_{2} / \mathrm{W}$

Figure 3.7: Transmission curves for longitudinal and shear waves in a nine layer reflector stack of (a) $\mathrm{SiO}_{2} / \mathrm{Ta}_{2} \mathrm{O}_{5}(\mathrm{c}=1.7)$ and (b) $\mathrm{SiO}_{2} / \mathrm{W}(\mathrm{c}=1.2)$. The solid and dotted lines indicate longitudinal and shear waves respectively. The vertical dotted lines indicate the frequency of optimization $\left(f_{R}=1.88\right.$ $\mathrm{GHz}$ ). The much lower transmission coefficients in fig. (b) compared to fig. (a) are related to high acoustic impedance ratio of $\mathrm{SiO}_{2} / W$ (refer to Table 3.5).

The improvements by the phase error approach as compared to the basic stop-band theory approach would be more pronounced if the velocity ratio deviates much from 2 . However, there is a trade-off: minimizing the longitudinal wave transmission is always at the cost of shear wave transmission. Therefore, depending on the user requirements a choice should be made between the design-approaches. A comparison of longitudinal ( $\left.T_{\text {long }}\right)$ and shear $\left(T_{\text {shear }}\right)$ transmission coefficients of a reflector stack designed with the basic stop-band theory approach (using eq.(3.10)) and improvements using the phase error approach (using eq.(3.23)) are given in Table 3.3.

We have also done an analysis varying the number of layers for a $\mathrm{SiO}_{2} / \mathrm{Ta}_{2} \mathrm{O}_{5}$ reflector stack retaining the periodicity of the stop-band theory stack configuration with a spacer. This is shown in Figure 3.8. As expected, it is found that a reflector stack with an odd number of layers has a better performance. This holds for any multilayer periodic system [21].

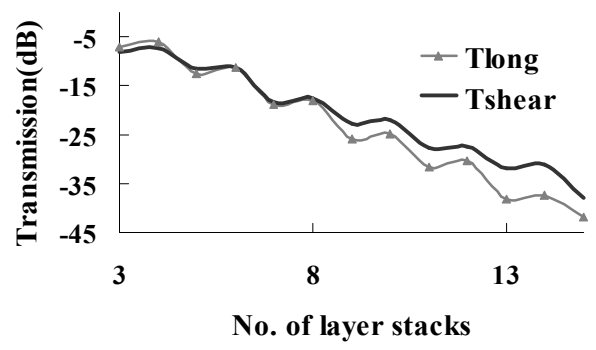

Figure 3.8:

Transmission vs. number of layer stacks for a nine layer $\mathrm{SiO}_{2} / \mathrm{Ta}_{2} \mathrm{O}_{5}$ optimized reflector stack obtained using the stop-band theory approach. 


\begin{tabular}{lccccc}
\hline \hline \multirow{2}{*}{ Stack } & \multicolumn{2}{c}{ Stop-band approach } & \multicolumn{3}{c}{ Phase error approach } \\
\cline { 2 - 6 } & $T_{\text {long(dB) }}$ & $T_{\text {shear }}(\mathrm{dB})$ & $c$ & $T_{\text {long(dB) }}$ & $T_{\text {shear }}(\mathrm{dB})$ \\
\hline $\mathrm{SiO}_{2} /$ & -25.7 & -22.8 & 1.7 & -27.4 & -17.3 \\
$\mathrm{Ta}_{2} \mathrm{O}_{5}$ & & & & & \\
$\mathrm{SiO}_{2} /$ & -60.8 & -53.9 & 1.2 & -67.3 & -47.1 \\
$\mathrm{Pt}$ & & -56.6 & 1.2 & -70.5 & -35.2 \\
$\mathrm{SiO}_{2} /$ & -63.6 & -56.6 & & & \\
$\mathrm{~W}$ & & & & \\
\hline \hline
\end{tabular}

Table 3.3. The improvements in longitudinal and shear transmissions obtained by the phase error approach compared to the stop-band approach.

\subsection{Diffraction grating based approaches}

In this section, the problem of dual wave reflection is solved using the fact that the reflector stack is essentially a periodic structure and hence behaves much like a diffraction grating. Any arrangement which is equivalent in its action to a number of parallel equidistant slits of the same width is called a diffraction grating [22]. Treating the reflector stack as a grating, section 3.3.1 presents the diffraction grating method (DGM) for dual wave reflection. An alternative DGM (ADGM) which yields reduced layer thickness is described in section 3.3.2.

\subsubsection{The Diffraction Grating Method (DGM)}

The principle of diffraction grating is to get the wave reflected back by introducing some periodicities in the grating and thus cancelling the phase shift upon reflection. Figure 3.9 shows the reflection spectra of a nine layer $\mathrm{SiO}_{2} / \mathrm{Ta}_{2} \mathrm{O}_{5}$ quarter-wave reflector stack. Apart from the first-order reflections at $f_{\mathrm{R}}$ corresponding to $t=\lambda_{\text {long }} / 4$ for a longitudinal acoustic wave, we can also see higher order reflections [21]-[22] which correspond to the layer thickness:

$t=\frac{\lambda_{\text {long }}}{4}+m \cdot \frac{\lambda_{\text {long }}}{2}=(1+2 m) \cdot \frac{v_{\text {long }}}{4 f_{\mathrm{R}}}$,

where $\mathrm{m}$ is a non-negative integer.

Here we will make use of these higher order reflections. Adopting eq. (3.25), it is possible to have reflection (for a given $f_{\mathrm{R}}$ ) for waves with velocity $v_{\text {long }}$ at $m=m_{1}$ and for waves with velocity $v_{\text {shear }}$ at $m=m_{2}$ for a particular layer thickness $t$.

Consider a regular $(\mathrm{LH})^{n}$ layer stack. Let us look at a single layer, say a low impedance layer $\mathrm{L}$ having a thickness $t_{\mathrm{L}}$ which corresponds to a quarter wavelength. 


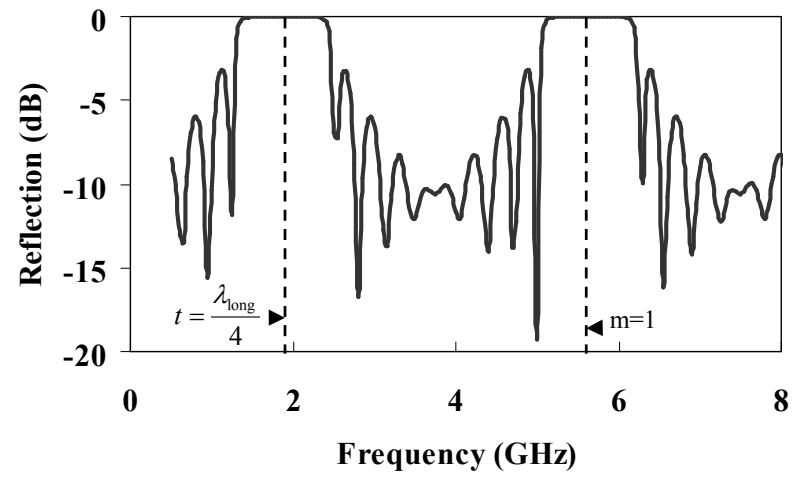

Figure 3.9: Reflection spectra of the longitudinal waves in a nine layer $\mathrm{SiO}_{2} / \mathrm{Ta}_{2} \mathrm{O}_{5}$ quarter-wave reflector stack.

Looking for the higher order reflections in the L layer so that both longitudinal and shear waves propagate, eq. (3.25) can be rewritten as:

$t_{\mathrm{L}}=\frac{\left(1+2 \cdot m_{1}\right) \cdot v_{\mathrm{L} . \text { long }}}{4 f_{\mathrm{R}}}=\frac{\left(1+2 \cdot m_{2}\right) \cdot v_{\mathrm{L} \text {.shear }}}{4 f_{\mathrm{R}}}$.

Working out eq. (3.26) gives:

$\frac{\left(1+2 \cdot m_{2}\right)}{\left(1+2 \cdot m_{1}\right)}=\frac{v_{\text {L.long }}}{v_{\text {L.shear }}}=K_{\mathrm{L}}$.

From eq. (3.27) it is deduced that two integers $m_{1}$ and $m_{2}$ are to be found that should satisfy the condition:

$m_{2}=K_{\mathrm{L}} \cdot m_{1}+\frac{\left(K_{\mathrm{L}}-1\right)}{2}$

Likewise, for the high impedance layer $\mathrm{H}$, we will have different velocities $v_{\text {Hlong }}$ and

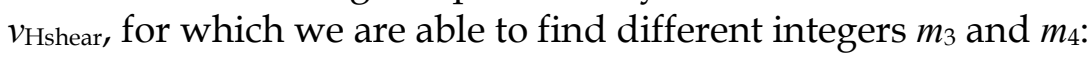

$t_{\mathrm{H}}=\frac{\left(1+2 \cdot m_{3}\right) \cdot v_{\mathrm{H} . \text { long }}}{4 f_{\mathrm{R}}}=\frac{\left(1+2 \cdot m_{4}\right) \cdot v_{\mathrm{H} . \text { shear }}}{4 f_{\mathrm{R}}}$.

From eq. (3.29) it is deduced that two integers $m_{3}$ and $m_{4}$ are to be found that should satisfy the condition:

$m_{4}=K_{\mathrm{H}} \cdot m_{3}+\frac{\left(K_{\mathrm{H}}-1\right)}{2}$

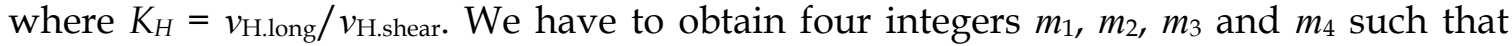
eqns. (3.28) and (3.30) are fulfilled. The values $m_{1}, m_{2}, m_{3}$ and $m_{4}$ are then substituted back 


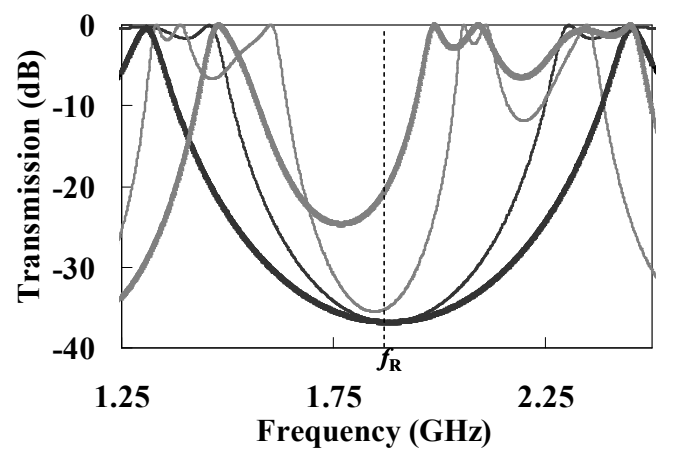

Figure 3.10 : $\quad$ Transmission curves of a five layer optimized reflector stack of $\mathrm{SiO}_{2} / \mathrm{W}$ using DGM (thin lines) and using ADGM (thick lines eq.(3.32)). The black and grey lines indicate longitudinal and shear transmission respectively. Optimization using ADGM shows an enhancement in bandwidth of longitudinal waves.

into eqns. (3.26) and (3.29) to obtain the layer thicknesses of the low and high impedance layers that efficiently reflect both longitudinal and shear waves.

The thickness for a $\mathrm{SiO}_{2} / \mathrm{Ta}_{2} \mathrm{O}_{5}\left(m_{1}=1, m_{2}=1.96, m_{3}=1, m_{4}=2.2\right)$ reflector stack is $2286 / 1764 \mathrm{~nm}$, calculated using this method with the described procedure. The values of $m_{2}$ and $m_{4}$ are not integers and hence have to be approximated to the nearest integer values. Figure 3.11 shows the modeled transmission curve of a nine layer $\mathrm{SiO}_{2} / \mathrm{Ta}_{2} \mathrm{O}_{5}$ $(2286 \mathrm{~nm} / 1764 \mathrm{~nm})$ mirror optimized at $1.88 \mathrm{GHz}$. Note that the optimum for shear waves is not at the exact proper frequency because $m_{4}$ is then not an integer.

It may be hard to fulfill conditions described by eqns. (3.28) and (3.30) for the cases in which $K_{\mathrm{L}}$ and/or $K_{\mathrm{H}}$ is close to 2 . The right hand side tends to be fractional numbers $\left(m_{1}=1, m_{2}=2.5, m_{3}=1, m_{4}=2.5\right)$ for small integer values of $m_{1}$ and $m_{3}$ in eqns. (3.28) and (3.30) respectively. This means that for $K_{\mathrm{L}}=K_{\mathrm{H}}=2, m_{2}$ and $m_{4}$ need to be approximated to the nearest integer. If we use a reflector stack with $\mathrm{SiO}_{2}$ as the low-impedance layer, $m_{1}=1$ and $m_{2}=2$ fulfills eq. (3.28) and an optimum at exact resonant frequency is expected. Some high-impedance materials will obey eq. (3.30) better than others.

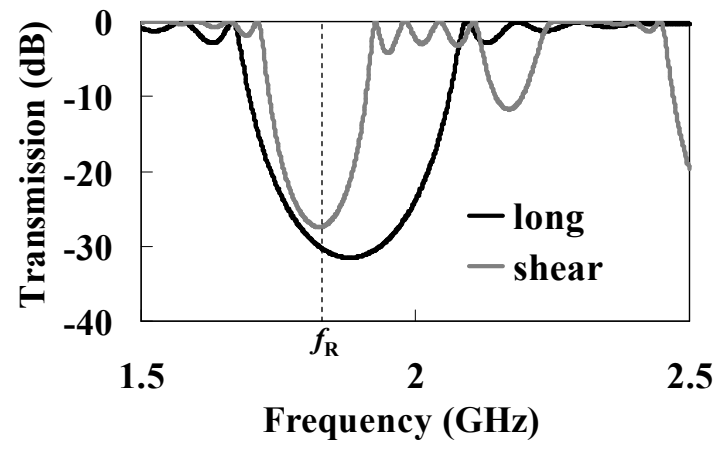

Figure 3.11 : $\quad$ Transmission curves of a nine layer optimized reflector stack of $\mathrm{SiO}_{2} / \mathrm{Ta}_{2} \mathrm{O}_{5}$. The transmission of longitudinal waves at resonance is about $-31.1 \mathrm{~dB}$ nearing quarter wave performance. The transmission of shear waves at resonance is about $-26.2 \mathrm{~dB}$. 


\begin{tabular}{ccc}
\hline \hline (a) $\mathrm{SiO}_{2} / \mathrm{W}(2285 \mathrm{~nm} / 718 \mathrm{~nm})$ \\
\hline \hline No. of layers & $\mathrm{T}_{\text {long }}(\mathbf{d B})$ & $\mathrm{T}_{\text {shear }}(\mathbf{d B})$ \\
\hline $\mathbf{3}$ & -19 & -12 \\
$\mathbf{5}$ & -37 & -22 \\
$\mathbf{7}$ & -55 & -32 \\
$\mathbf{9}$ & -73 & -41 \\
\hline \hline
\end{tabular}

\begin{tabular}{ccc}
\hline \hline (b) SiOC /Pt $(\mathbf{9 3 5} \mathbf{~ n m} / \mathbf{5 5 5} \mathbf{~ n m})$ \\
\hline \hline No. of layers & $\mathbf{T}_{\text {long }}(\mathbf{d B})$ & $\mathbf{T}_{\text {shear }}(\mathbf{d B})$ \\
\hline \hline 3 & -36 & -24 \\
5 & -63 & -38 \\
7 & -89 & -53 \\
9 & -116 & -69 \\
\hline \hline
\end{tabular}

Table 3.4 Calculated longitudinal and shear transmissions for varying number of layers of an optimized acoustic mirror at $f_{r}=1.88 \mathrm{GHz}$

It is concluded from the transmission line model calculations that the diffraction grating method gives best optimized stacks when $K_{\mathrm{L}}$ and $K_{\mathrm{H}}$ deviate from 2 by $15 \%$ or more. Nevertheless, the layer thicknesses obtained by the diffraction grating approach are relatively thick compared to those obtained by the stop-band theory approach. An alternative thickness optimization approach applicable for the stacks with a high impedance contrast, which results in reduced layer thickness, is discussed in section 3.3.2.

\subsubsection{An Alternative Diffraction Grating Method (ADGM)}

In this section, an alternative diffraction grating method [24] is presented. Following the discussions in section 3.3.1, for the minimum integer values of $m\left(m_{1}=m_{3}=1\right)$ in eqns. (3.26) and (3.29), the thicknesses in terms of longitudinal wavelength will be:

$t_{\mathrm{L}}=\frac{3 \cdot v_{\mathrm{L} . \text { long }}}{4 f_{\mathrm{R}}}=\frac{3 \cdot \lambda_{\mathrm{L} . \text { long }}}{4}$,

$t_{\mathrm{H}}=\frac{3 \cdot v_{\mathrm{H} . \text { long }}}{4 f_{\mathrm{R}}}=\frac{3 \cdot \lambda_{\mathrm{H} . \text { long }}}{4}$.

However, if the impedance contrast between the low impedance layer and high impedance layer is large enough, once could try matching fundamental reflection of one layer with the first higher order reflection of other layer. Consequently it is possible to achieve dual reflection if:

$t_{\mathrm{L}}=3 \cdot \frac{\lambda_{\mathrm{L} \text {.long }}}{4}, t_{\mathrm{H}}=\frac{\lambda_{\mathrm{H} \text {.long }}}{4}$,

or 
$t_{\mathrm{L}}=\frac{\lambda_{\mathrm{L} \text {.long }}}{4}, t_{\mathrm{H}}=3 \cdot \frac{\lambda_{\mathrm{H} . \text { long }}}{4}$.

In terms of transmission, the stacks designed by eqns. (3.32) or (3.33) are equally effective. If the low impedance (e.g.oxide) layer is thicker, this ADGM design approach can automatically end up in type I dispersion, the preferred dispersion type for frame region functioning [3] (For more details about dispersion types, refer section 2.5.). Hence let us go for the first choice, eq. (3.32). In this case the total thickness of the stack is now reduced compared to matching higher order reflection in both the layers given by eqns. (3.26) and (3.29).

The dual reflection in these layers is achieved as follows. The corresponding phase drops in the layers obtained by eq. (3.32) are:

$\phi_{\text {L.long }}=t_{\mathrm{L}} \cdot\left(\frac{2 \pi}{\lambda_{\text {L.long }}}\right)=3 \cdot \frac{\lambda_{\text {L.long }}}{4}\left(\frac{2 \pi}{\lambda_{\text {L.long }}}\right)=\frac{3 \pi}{2}$,

and

$\phi_{\text {H.long }}=t_{\mathrm{H}} \cdot\left(\frac{2 \pi}{\lambda_{\text {H.long }}}\right)=\frac{\lambda_{\text {H.long }}}{4}\left(\frac{2 \pi}{\lambda_{\text {H.long }}}\right)=\frac{\pi}{2}$.

The ratio of the longitudinal phase drops, $\phi_{\mathrm{L} . \text { long }} / \phi_{\mathrm{H} \text {.long }}$ in these layers is 3 (Note that $c$ given by eq. (3.7) is defined as $\phi_{\mathrm{L} \text {.long }} / \phi_{\mathrm{H} \text {.long }}$ because of the $\mathrm{LcH}$ configuration considered). This implies $c=3$ which corresponds to a 3L.H configuration [15].

A similar analysis can be done writing the equations in terms of shear waves. We know that $\lambda_{\text {shear }}=\lambda_{\text {long }} / 2$. Starting with eq.(3.32), the layer thicknesses in terms of shear wave velocities are then given by:

$t_{\mathrm{L}}=3 \cdot \frac{\lambda_{\mathrm{L} . \text { hear }}}{2}, t_{\mathrm{H}}=\frac{\lambda_{\mathrm{H} \text {.shear }}}{2}$.

The corresponding phase drops in these layers can be obtained by working out the eqns. (3.34) and (3.35) in terms of shear wavelength as well. The ratio of the shear phase drops, $\phi_{\mathrm{L} \text {.shear }} / \phi_{\mathrm{H} \text {.shear }}$ in these layers is also 3 . Hence the reflection condition for $3 \mathrm{~L} \cdot \mathrm{H}$ configuration is simultaneously fulfilled for both longitudinal and shear waves.

The design procedure was applied for various material combinations. In the case of high impedance contrast material combinations like $\mathrm{SiO}_{2} / \mathrm{W}, \mathrm{SiO}_{2} / \mathrm{Pt}, \mathrm{SiOC} / \mathrm{Pt}$ and $\mathrm{SiO}_{2} / \mathrm{TiN}$ the ADGM optimized layer stacks show efficient reflection of both longitudinal and shear waves. In Table 3.4 the transmissions are summarized for various material combinations and number of layers. 


\begin{tabular}{llll}
\hline \hline $\mathrm{L} / \mathrm{H}$ & $\mathrm{Z}_{\mathrm{L}}$ & $\mathbf{Z}_{\mathrm{H}}$ & $\mathrm{Z}_{\mathrm{H}} / \mathbf{Z}_{\mathrm{L}}$ \\
& $\left(\mathbf{k g} / \mathbf{m}^{2} \mathbf{s}\right)$ & $\mathbf{( k g / \mathbf { m } ^ { 2 } \mathbf { s } )}$ & \\
\hline $\mathrm{SiOC} / \mathbf{P t}$ & $4.22 \cdot 10^{6}$ & $8.94 \cdot 10^{7}$ & 21.2 \\
$\mathrm{SiO}_{2} / \mathbf{W}$ & $1.23 \cdot 10^{7}$ & $1.02 \cdot 10^{8}$ & 12 \\
$\mathrm{SiO}_{2} / \mathbf{P t}$ & $1.23 \cdot 10^{7}$ & $8.94 \cdot 10^{7}$ & 7.2 \\
$\mathrm{SiO}_{2} / \mathbf{T i N}$ & $1.23 \cdot 10^{7}$ & $5.37 \cdot 10^{7}$ & 4.4 \\
$\mathrm{SiO}_{2} / \mathrm{AlN}$ & $1.23 \cdot 10^{7}$ & $3.60 \cdot 10^{7}$ & 2.9 \\
$\mathrm{SiO}_{2} / \mathrm{Ta}_{2} \mathrm{O}_{5}$ & $1.23 \cdot 10^{7}$ & $3.08 \cdot 10^{7}$ & 2.5 \\
\hline \hline
\end{tabular}

Table 3.5. Impedance ratio for various material combinations. Data obtained from [23].

ADGM works for most of the dielectric/metal stacks and also for some dielectric/dielectric stacks (eg. $\mathrm{SiO}_{2} / \mathrm{AlN}$ ). However for some dielectric/dielectric stacks with low impedance contrasts like $\mathrm{SiO}_{2} / \mathrm{Ta}_{2} \mathrm{O}_{5}$ only the higher order reflection match (DGM) applies. The impedance contrasts for various material combinations considered here are summarized in Table 3.5 [23].

The modeling results imply that for the material combinations with quite high impedance contrast $(\geq 4)$, ADGM should be adopted to fulfill the demands of dual reflection. If the impedance contrast is $\leq 4$, DGM should be adopted. The advantages of ADGM are a reduced layer thickness and an improved bandwidth (ref. Figure 3.10). The DGM leads to relatively thick layers if the wave velocities in the $\mathrm{L}$ and $\mathrm{H}$ layers are quite high.

\subsection{D FEM simulations}

In this section, 2D FEM simulations of the SMRs with the newly designed reflector stacks are carried out to validate the design approaches. SMRs with two different reflector stacks are simulated: $\mathrm{SiO}_{2} / \mathrm{Ta}_{2} \mathrm{O}_{5}$ and $\mathrm{SiO}_{2} / \mathrm{W}$ stacks. A 2-D FEM physical model of the SMR with the stacks designed using various approaches was developed using the Comsol Multiphysics tool [25]. Half structure simulations were done exploiting symmetry with continuous layers for the reflector and bottom electrode. The discontinuous layers were the top electrode and the piezoelectric layer (with free edge boundaries). At right hand side a fixed $\left(U_{x}=U_{z}=0\right)$ boundary condition was applied and at the left hand side a symmetric boundary condition $\left(U_{x}=0, \partial U_{z} / \partial x=0\right)$ was applied.

Figure 3.12 shows the simulation results of the vertical displacement profile of longitudinal and shear waves at resonant frequency, $f_{\mathrm{R}}$ in an SMR with a nine layer $\mathrm{SiO}_{2} / \mathrm{Ta}_{2} \mathrm{O}_{5}$ reflector stack using (a) a quarter-wave stack, (b) and (c) shear optimized stacks designed using the theory described in section 3.2, and (d) a shear optimized stack designed using the diffraction grating method (DGM) (section 3.3.1). The stacks are optimized at $f_{\mathrm{R}}=1.88 \mathrm{GHz}$. As the average velocity ratio as described in eq. (3.14) is close to 2 in the case of the $\mathrm{SiO}_{2} / \mathrm{Ta}_{2} \mathrm{O}_{5}$ stack, the basic stop-band theory approach also 


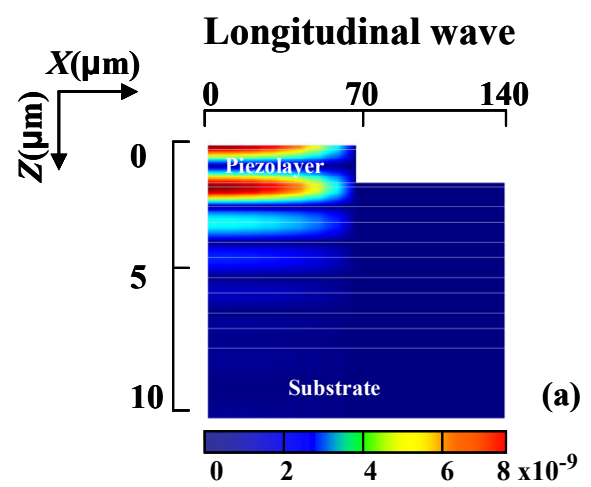

\section{Shear wave}
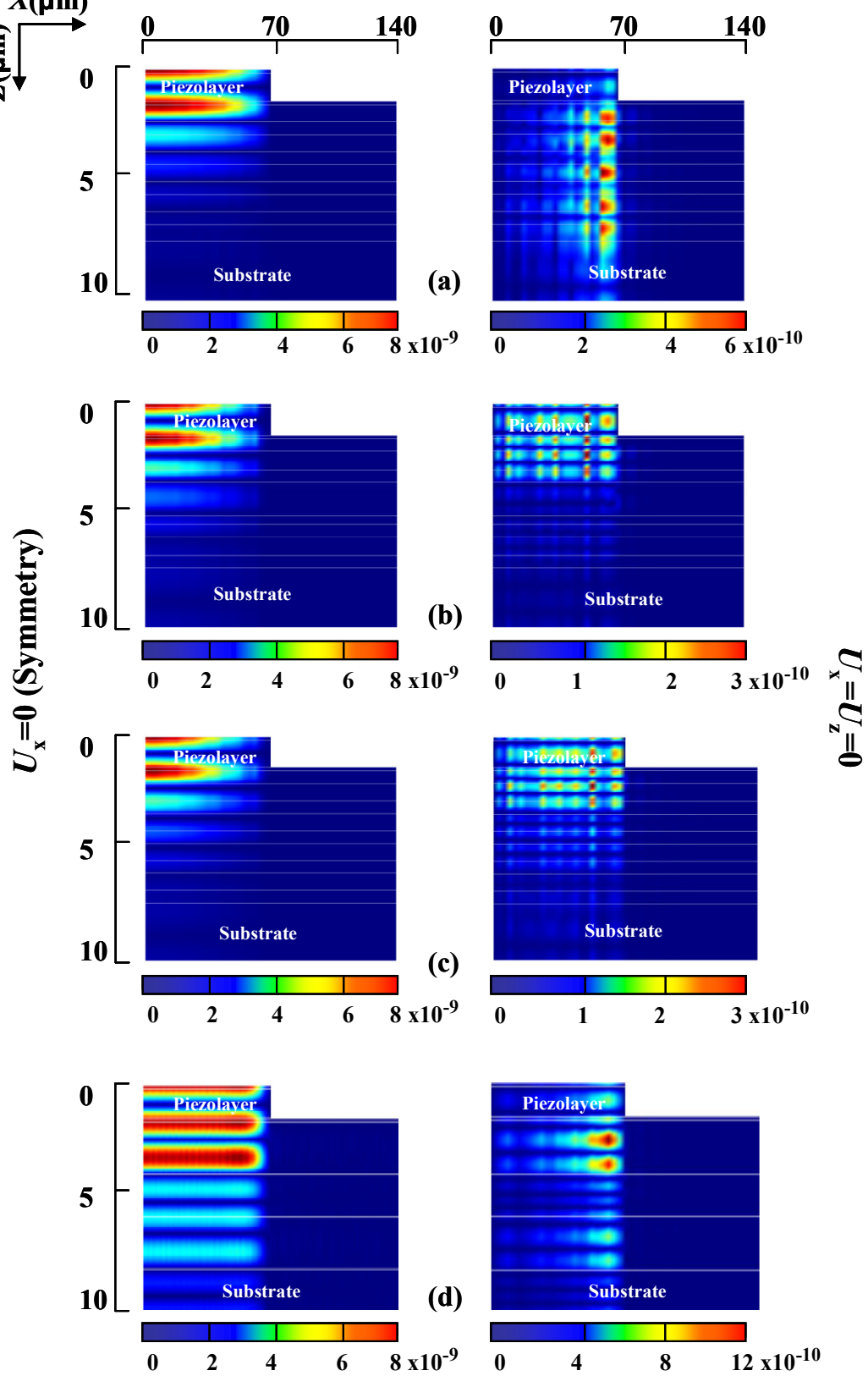

Figure 3.12:

2D FEM results for displacement of longitudinal waves (left) and shear waves (right) at resonant frequency in an SMR with (a) a quarter wave stack $(762 \mathrm{~nm} / 588 \mathrm{~nm})$, (b) shear optimized stack using stop-band theory, $c=1.73$, RS:(559/862/559/431/1118/431/559/862/559(nm), Spacer: 431/1118/431) (c) shear optimized stack using phase error approach, $c=1.7(617 \mathrm{~nm} / 810 \mathrm{~nm})$ and $(d)$ shear optimized stack using diffraction grating approach $(2286 \mathrm{~nm} / 1764 \mathrm{~nm})$ for a nine layer $\mathrm{SiO}_{2} / \mathrm{Ta}_{2} \mathrm{O}_{5}$ reflector stack. Plane of symmetry is defined at the left edge of the device. Note that in fig (d) results are shown only for $10 \mu \mathrm{m}$ depth for comparison; the simulation is for 9 layers. Boundary conditions at bottom and top are assumed to be fixed $\left(U_{x}=U_{z}=0\right)$. 


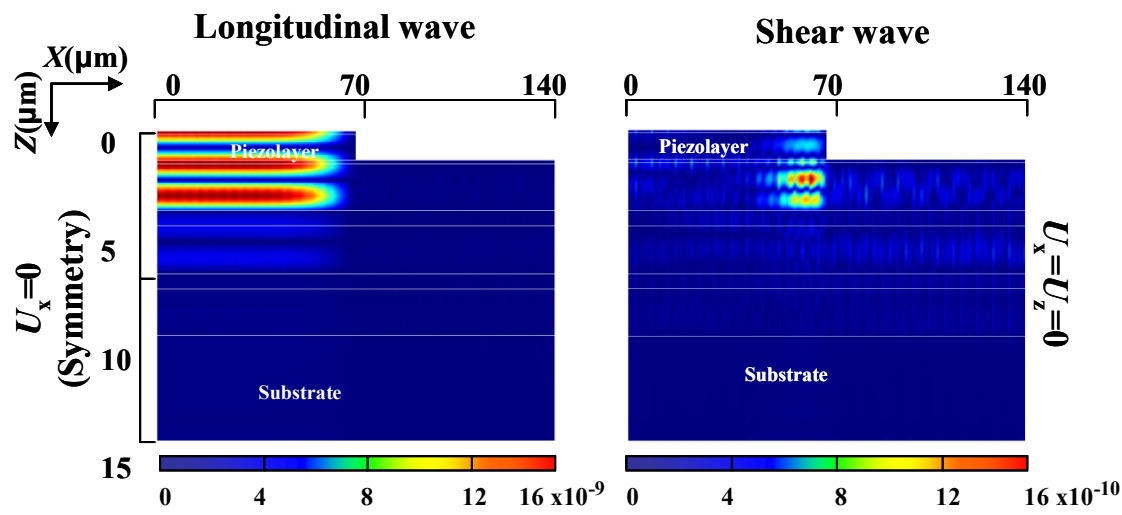

Figure 3.13 : $\quad 2 D$ FEM results for displacement of longitudinal waves (left) and shear waves(right) at resonant frequency in an $\mathrm{SMR}$ with a five layer $\mathrm{SiO}_{2} / W(2285 \mathrm{~nm} / 718 \mathrm{~nm})$ reflector stack obtained from the ADGM. Plane of symmetry is defined at the left edge of the device. Boundary conditions at bottom and top are assumed to be free $\left(U_{x}=U_{z}=0\right)$.

holds well in this case. The optimized thicknesses were implemented in the spacer configuration described in [14]. It is seen from the figures that in the resonator with a conventional quarter-wave mirror, shear waves are leaking into the substrate whereas in the case of shear-optimized stacks, leakage of shear waves into the substrate is suppressed. In the case of Figure 3.12 (b), the shear optimized stack obtained from the stop-band theory with spacer, the shear waves penetrate deeper into the mirror till the middle spacer layer. This explains why the shear transmission is mainly sensitive to the thickness of the middle spacer layer as mentioned in section 3.2.1.

An SMR with a five layer $\mathrm{SiO}_{2} / \mathrm{W}(2285 \mathrm{~nm} / 718 \mathrm{~nm})$ stack optimized at $1.88 \mathrm{GHz}$ with an alternative diffraction grating method (ADGM) (section 3.3.2) was also simulated and the displacement profiles were compared. Figure 3.13 shows the vertical displacement profile of longitudinal and shear waves at $f_{R}$ in such an SMR. Note that the shear wave has a maximum displacement in the top-most mirror oxide as reported in [3]. The $\mathrm{SiO}_{2} / \mathrm{W}$ ADGM stack gives a wide longitudinal displacement profile which also indicates the effectiveness of the contrast ratio as seen from Figure 3.13. A more detailed discussion of $\mathrm{SiO}_{2} / \mathrm{W}$ stacks obtained with various approaches is presented in chapter 5 .

Figure 3.12 and Figure 3.13 are an illustration of the effect of the stack obtained with the proposed design approaches discussed in previous sections. Although a visual interpretation of the effectiveness of the reflector stack is presented in this chapter, it is possible to compute electrical impedance characteristics and hence $Q$ values from 2D FEM simulations (see chapter 5).

\subsection{Comparison of the design approaches}

The approaches described in sections 3.2 and 3.3 are based on velocity ratios. If the velocity ratios $K_{\mathrm{L}}$ and $K_{\mathrm{H}}$ are close to 2 , the reflector stack can be designed using the basic stop-band approach and can be implemented with the spacer configuration. In the case of which a trade-off is to be found between longitudinal and shear transmission, the optimized thicknesses can be obtained by varying $c$ following the phase error approach described in section 3.2.2. The layer thicknesses calculated using the diffraction grating 


\begin{tabular}{lccc}
\hline \hline \multicolumn{1}{c}{ Design approach } & Tlong $(\mathrm{dB})$ & Tshear $(\mathrm{dB})$ & Stack thickness $(\mu \mathrm{m})$ \\
\hline Quarter-wave stack & -31.5 & -0.59 & 6.15 \\
Stop-band theory & -25.7 & -22.8 & 5.94 \\
Phase error approach & -27.4 & -17.3 & 6.325 \\
Diffraction grating & -31.1 & -26.2 & 18.48 \\
\hline \hline
\end{tabular}

Table 3.6 Comparison of three approaches for a nine layer $\mathrm{SiO}_{2} / \mathrm{Ta}_{2} \mathrm{O}_{5}$ stack at resonance frequency.

method described in section 3.3.1 are generally above $1 \mu \mathrm{m}$ for the resonant frequency of $1.88 \mathrm{GHz}$ for the same number of layers. However, this method gives best results when the average velocity ratio (as described by eq. (3.14)) deviates from the usual value of 2 by $15 \%$ or more. On the other hand, the stop-band approach performs best when the average velocity ratio deviates from 2 by $15 \%$ or less, resulting in a phase error of less than $20 \%$. Hence the diffraction grating approach complements the basic stop band approach. If there are no constraints for the fabrication of thicker layers, the diffraction grating method gives a straight forward guideline for the stack design.

Table 3.6 summarizes the comparison of transmission of longitudinal and shear waves at $f_{\mathrm{R}}$ in a nine layer $\mathrm{SiO}_{2} / \mathrm{Ta}_{2} \mathrm{O}_{5}$ acoustic mirror designed with three approaches. It can be concluded that the transmission of longitudinal waves is near quarter-wave performance for the SMR with stacks obtained from the diffraction grating method (DGM). Using an ADGM designed five layer $\mathrm{SiO}_{2} / \mathrm{W}$ stack, a transmission of $-37 \mathrm{~dB}$ is maintained for longitudinal waves whereas shear waves reach value below $-22 \mathrm{~dB}$ (see Figure 3.10).

The diffraction grating approaches can be concluded as variations of basic optical filter configurations discussed in [15]. For minimum integer values, the DGM stack thickness given by eq. (3.31) is actually a (3H) (3L) stack, a variation of the basic QW stack (HL). Hence this stack emulates properties of a basic QW stack w.r.t. longitudinal transmission as shown in Table 3.6. The shear reflection property of the stack is due to the grating action explained in section 3.3. An attempt was also made to map the thickness of the DGM stack back to the $c$ parameter of the phase error approach. However, non-realistic $c$ values were obtained.

Similarly the ADGM stack thickness given by eq. (3.32) or eq. (3.33) is analogous to (3L) $\mathrm{H}$ or $\mathrm{L}(3 \mathrm{H})$ configurations respectively in optical filters [15]. Because of the fact that $\lambda_{\text {long }}=2 . \lambda_{\text {shear }}[3],(3 \mathrm{~L}) \mathrm{H}$ or $\mathrm{L}(3 \mathrm{H})$ automatically satisfies the reflection condition for both longitudinal and shear waves simultaneously. Note that ADGM stacks are similar to the QW stacks with increased top-oxide with the exception that the alternate low impedance layers are $3 \mathrm{QW}$ thick. To put it in a broader perspective, the QW stack with increased top-oxide can be considered as a special case of ADGM stacks as addressed in chapter 5 .

\subsection{Conclusions}

In this chapter, design approaches, derived from optics, have been presented for optimizing dual reflection of longitudinal and shear waves in solidly mounted BAW resonators. Several design approaches have been discussed, some based on the stop- 
band theory and the others on the diffraction grating method, all involving the velocity ratio of longitudinal to shear wave in the reflector stack layers. A phase error approach is presented that allows the designer to find a trade-off between longitudinal and shear reflection in the stack.

Using the diffraction grating method, a near quarter-wave performance is maintained for longitudinal waves whereas shear waves reach minimum transmission below $-26 \mathrm{~dB}$. However, this design does necessitate relatively thick layers. Compared to DGM, ADGM resulted in a reduced layer thickness and an improved bandwidth. In the case of stop-band theory based approaches, the improvement in bandwidth was not as significant as in the case of ADGM stacks.

The results obtained from the design approaches have been verified with 2D FEM simulations. A comparison of transmission characteristics from the analytical model and displacement profile from FEM simulations has been carried out; the trend in the model and the simulations were found to be in agreement. FEM simulations confirm that the leakage of shear waves into the substrate is suppressed in all shear-optimized stacks from the presented approaches. A comparison of the design approaches has also been presented.

The approaches has been demonstrated for different material combinations and in all cases a minimum transmission of $-25 \mathrm{~dB}$ and $-20 \mathrm{~dB}$ at resonance frequencies were obtained for longitudinal and shear waves respectively, for various practical reflector material combinations. Adaptability of these approaches makes it a potential design guide-line for the devices with minimized acoustic losses into the substrate, and consequently a high acoustic quality factor.

The thickness of the mirror stack affects the dispersion response of the resonator. Therefore, we will analyze the dispersion behavior of such optimized stacks in chapter 4 . The experimental verification of the theories presented in this chapter is investigated in chapter 5 .

\section{References}

[1] S. Marksteiner, J. Kaitila, G. G. Fattinger and R. Aigner, "Optimization of acoustic mirrors for Solidly Mounted BAW resonators," Proc. IEEE Ultrasonics Symposium, pp. 329-332, 2005.

[2] S. Marksteiner, G. G. Fattinger, R. Aigner and J. Kaitila, Acoustic Reflector for a BAW resonator providing specified reflection of both shear wave and longitudinal waves, US patent: 006933807B2., Aug. 2005.

[3] G. G. Fattinger, S. Marksteiner, J. Kaitila, and R. Aigner, "Optimization of acoustic dispersion for high performance thin film BAW resonators," Proc. IEEE Ultrasonics Symposium, pp. 1175-1178, 2005.

[4] J. W. Lobeek, R. Strijbos, A. B. M. Jansman, N. X. Li, A. B .Smolders and N. Pulsford, "High- $Q$ BAW resonator on $\mathrm{Pt} / \mathrm{Ta}_{2} \mathrm{O}_{5} / \mathrm{SiO}_{2}$-based reflector stack," Proc. IEEE Microwave Symposium, pp. 2047-2050, 2007. 
[5] R. Strijbos, A. B. M. Jansman, J. W. Lobeek, N. X. Li and N. Pulsford, "Design and characterization of high- $Q$ Solidly-Mounted Bulk Acoustic Wave filters," Proc. IEEE Electronic components and technology conference, pp. 169-174, 2007.

[6] S. Jose, A. B. M. Jansman, R. J. E. Hueting and J. Schmitz, “Optimized reflector stacks for solidly mounted bulk acoustic wave resonators," IEEE Trans. on Ultrasonics Ferroelectric and Frequency Control, vol. 57, no. 12, pp. 2753-2763, 2010.

[7] K. M .Lakin, G. R. Kline and K. T. McCarron, "High- $Q$ microwave acoustic resonators and filters," IEEE Trans. Microwave Theory and Techniques, vol. 41, no. 12, pp. 2139-2146, 1993.

[8] W. E. Newell, "Face-Mounted Piezoelectric Resonators," Proc. IEEE, vol. 53, no. 6, pp. 575$581,1965$.

[9] K. M. Lakin, K. T. McCarron, and R. E. Rose, "Solidly mounted resonators and filters," Proc. IEEE Ultrasonics Symposium, pp. 905-908, 1995.

[10] H. P. Loebl, C. Metzmacher, R.F. Milsom, P. Lok, F. Van straten and A. Tuinhout, "RF bulk acoustic resonators and filters," Kluwer Journal of Electroceramics, vol. 12, pp. 109-118, 2004.

[11]M. J. S. Lowe, "Matrix techniques for modeling ultrasonic waves in multilayered media," IEEE Trans. Ultrason. Ferroelectr. Freq. Control, vol. 42, no. 4, pp. 525-541, 1995.

[12] Mathcad, Mathsoft Engineering \& Education, Inc., Version 13, 2005.

[13]J. Kaitila, "Review of wave propagation in BAW thin film devices progress and prospects," Proc. IEEE Ultrasonics Symposium, pp. 120-129, 2007.

[14] S. Jose, A. B. M. Jansman and R. J. E. Hueting, "A design Procedure for an acoustic mirror providing dual reflection of longitudinal and shear waves in Solidly Mounted BAW resonators (SMRs)," Proc. IEEE Ultrasonics Symposium, September, pp. 2111-2114, 2009.

[15] Optics of thin films, Z.Knittl, 1st edition, John Wiley \& Sons, pp. 66-67, 86-103,1976.

[16] Lord Rayleigh, "On the reflection of light from a regularly stratified medium," Proc. Roy. Soc. (London), vol. A 93, 565, 1917.

[17] G. Pei-fu and Z. Zhen-rong, "Design of non-polarizing thin film edge filters" Journal of Zhejiang University Science A, vol. 7,no. 6, pp. 1037-1040, 2006.

[18]A. Thelen, Design of optical interference coatings, Mc Graw-Hill Optical and ElectroOptical Engineering Series, 1988.

[19]S. G. Alekseev, Yu. V. Gulyaev, G.D. mansfeld and V. I. Pustovoit, "Propagation of acoustic waves through periodic structures containing phase non-regularities," IEEE Proc. Ultrasonics Symposium, pp. 2124-2127, 2005.

[20]G. G. Fattinger, "BAW resonators design considerations -An overview," Proc. IEEE International Frequency Control Symposium, pp. 762-767, 2008.

[21] Optics, Eugen Hecht, 3rd edition, Addison Wesley Longman Inc., pp. 422, 487-494, 1998.

[22] Fundamentals of Optics, Francis A. Jenkins and Harvey E. White, 4th edition, McGraw-Hill International Editions, pp. 355-356,380, 1981.

[23] Private communication with A. B. M. Jansman, NXP Semiconductors, Eindhoven (NL).

[24] S. Jose, R. J. E. Hueting and A. B. M. Jansman, "Acoustic dispersion of Solidly Mounted Resonators with an optimized reflector stack for dual wave reflection," Proc. IEEE Ultrasonics Symposium, pp. 291-294, 2010. 
[25] Comsol Multiphysics, version 3.5, 2009 (www.comsol.com). 



\section{Acoustic dispersion of SMRs with optimized reflector stacks}

This chapter presents the influence of the reflector stack design on the acoustic dispersion of solidly mounted resonators (SMRs). Depending on the reflector stack design approaches discussed in chapter three, the resonators exhibit dispersion type I or type II. A rule of thumb for flipping the dispersion to type I, the preferred dispersion type for adopting a frame region, is proposed and discussed. 2D FEM simulations demonstrate that the rule of thumb can be applied to both FBARs and SMRs. 
In chapter 3, various design approaches for optimizing the reflector stack for dual wave reflection have been discussed. The effectiveness of such a stack can be verified experimentally by extracting the quality factor of the resonator. However, to extract a reliable quality factor, the spurious resonances should be suppressed in the electrical characteristics. In practice, the suppression of spurious modes by the use of a frame region is a well accepted scheme, but this demands the dispersion relation of the resonator to be type I.

In this chapter, we analyze the acoustic dispersion relation of the SMRs employing the reflector stacks obtained with the proposed design approaches. The dispersion type has been determined from the impedance curves obtained from analytical calculations and confirmed by 2D FEM eigen frequency simulations. After analyzing the confinement of longitudinal and shear waves from 2D FEM simulations, we further propose a rule of thumb for flipping the dispersion curve to type I for SMRs having a type II piezoelectric material. The simulations demonstrate that the rule of thumb can be applied to both FBARs and SMRs. Some guidelines about applicability of the rule are addressed in the discussion section. The chapter summary is presented in the conclusion section. This chapter presents the work described in [1] .

\subsection{Influence of reflector stack design on acoustic dispersion}

It is generally accepted that one of the advantages of a Solidly Mounted Resonator (SMR) over a Film Bulk Acoustic wave Resonator (FBAR) is that the acoustic dispersion of the former can be modified by an educated design of the reflector stack. The SMR provides an additional degree of freedom to engineer the dispersion curves of the acoustic branches, compared to the FBAR [1]-[5]. Aluminum Nitride (AIN) based resonators without any precautions (e.g. an FBAR) would usually end up in a type II behavior, as AlN is a type II material [2], [3].

The dispersion relation in the case of a single piezoelectric layer is governed by the material parameter called the Poisson ratio of the piezoelectric material [2]. The acoustic velocity of shear $\left(v_{\mathrm{S}}\right)$ and longitudinal waves $\left(v_{L}\right)$ in the piezoelectric material are related to the Poisson ratio o according to [2]:

$$
\frac{v_{\mathrm{S}}}{v_{\mathrm{L}}}=\sqrt{\frac{2 \sigma-1}{2 \sigma-2}} \text {. }
$$

However, the acoustic properties of the mirror can be tailored by the number of layers and by the choice of materials and their thicknesses in the underlying reflector stack [6][9]. This makes it possible to achieve type I response in an SMR by the proper modification of the stack layers, despite of the type II piezoelectric material used here. Type I dispersion is critical for the functioning of the frame region [2], [6], [10], [11].

The dispersion type can be distinguished by the relative position of longitudinal main resonance mode (TE1) and the second shear resonance mode (TS2). A more detailed 


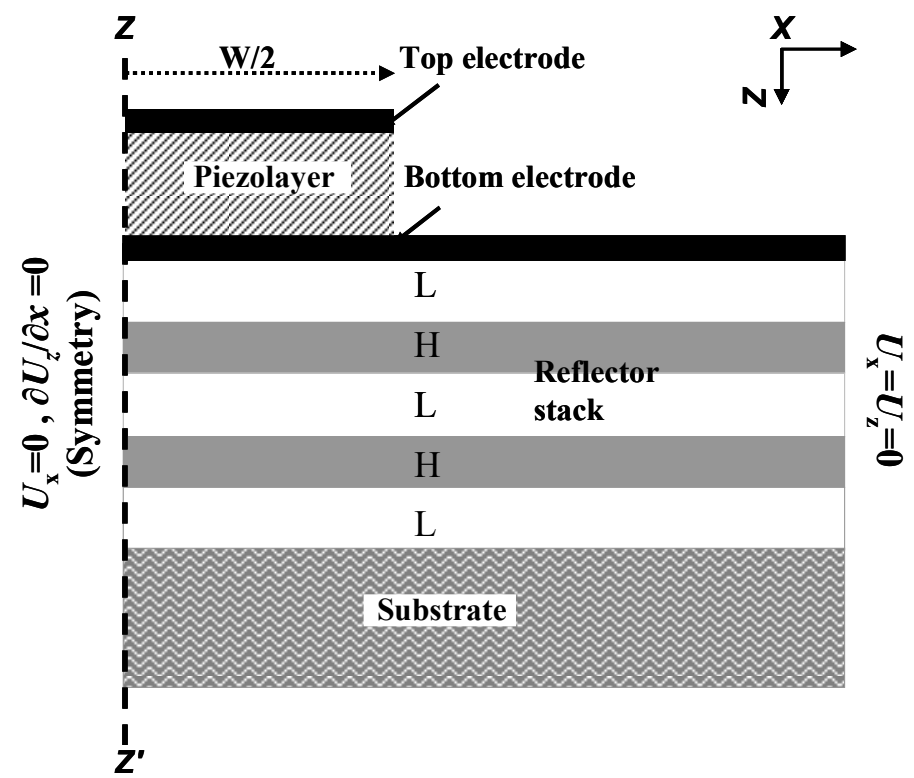

Figure 4.1: $\quad$ Schematic cross section of an SMR indicating the boundary conditions. The axis of symmetry assumed in the $2 D$ FEM simulations is indicated by the $Z-Z^{\prime}$ line. Boundary conditions at bottom and top are assumed to be free.

discussion about dispersion curves, definitions of TE1, TS1 and TS2 is presented in chapter 2. If $f_{\mathrm{TE} 1}>f_{\mathrm{TS} 2}$ the dispersion is type I, and type II otherwise [2], [3]. Moreover, in the case of type I dispersion, $f_{\text {TE1 }}$ is related to the dispersion branch of positive slope whereas in type II dispersion it is negative [2], [6], [10], [11].

The dispersion response of the SMRs, with shear optimized stacks as discussed in chapter 3, has been verified by checking the slope [10] of the dispersion branch of the longitudinal main resonance and also confirmed by the relative position of TE1 and TS2 from the impedance curves [3]. 2D FEM simulations [12] were performed to obtain the dispersion curves. A schematic cross section of the simulated device with the applied boundary conditions (BC) is shown in Figure 4.1. Simulations were done by using the Z$Z^{\prime}$ line as the axis of symmetry with continuous layers for the reflector and bottom electrode. The discontinuous layers were the top electrode and the piezoelectric layer each with free edge boundaries. At the right hand side, the vertical and horizontal displacements were assumed to be zero $\left(U_{x}=U_{z}=0\right)$, and at the left hand side, a symmetric boundary condition $\left(U_{x}=0, \partial U_{z} / \partial x=0\right)$ were applied. The geometry has no damping (ref. Appendix A ) at the right edge as it is not going to affect the position of the resonances. The continuous layers extend to a distance of $50 \mu \mathrm{m}$ outside the resonator area, making the modes insensitive to the actual geometry of the device [13]. The fundamental eigen mode of the resonator has half a lateral wavelength trapped within the resonator width $\mathrm{W}$. The lateral wave number $k_{x}$ can be calculated according to [14], [15]: 


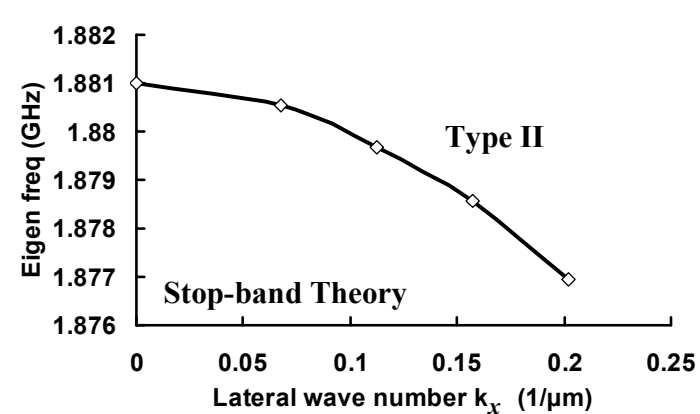

(a)

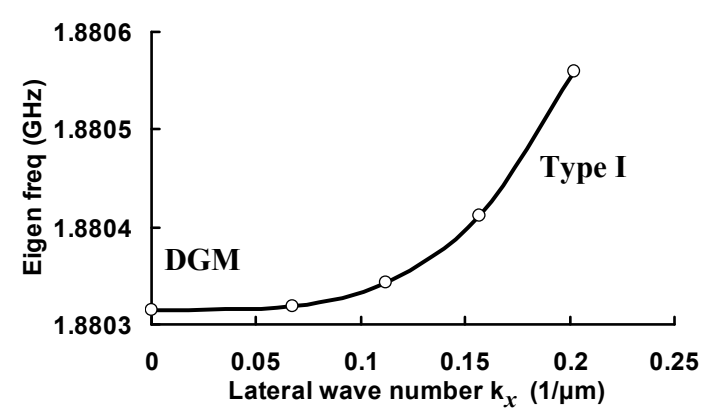

(c)

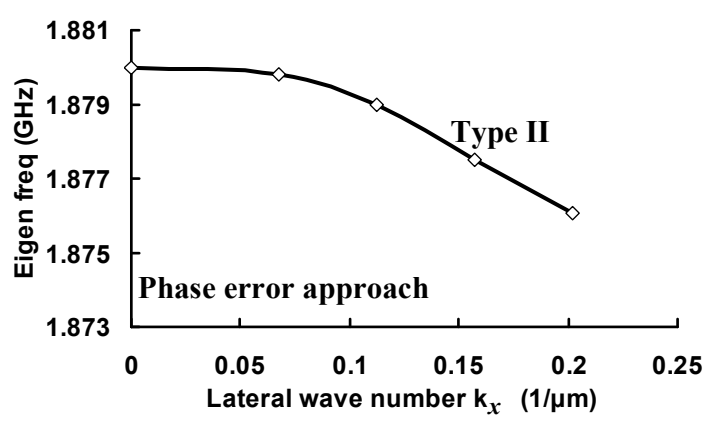

(b)

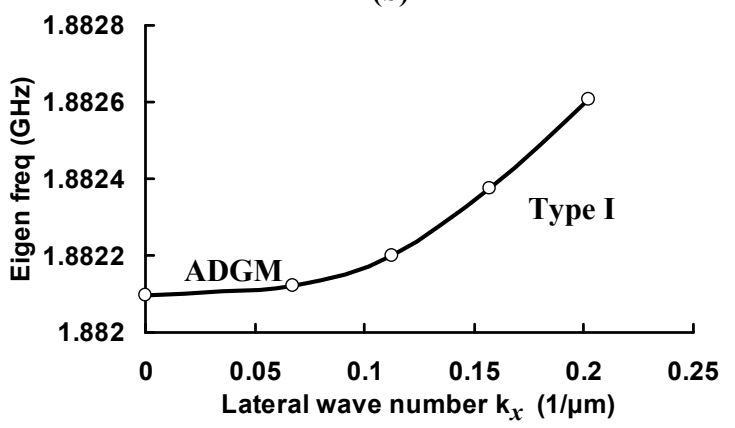

(d)

Figure 4.2:

Dispersion curves obtained from $2 D$ FEM eigen frequency simulations for resonators with a nine layer $\mathrm{SiO}_{2} / \mathrm{Ta}_{2} \mathrm{O}_{5}$ shear-optimized stack at $\approx 1.88 \mathrm{GHz}$ obtained with (a) stopband theory including spacer $(559 \mathrm{~nm} / 862 \mathrm{~nm})$ (b) phase error approach $(617 \mathrm{~nm} / 810 \mathrm{~nm})$ (c) diffraction grating method (DGM) $(2285 \mathrm{~nm} / 1765 \mathrm{~nm})$ and (d) a five layer $\mathrm{SiO}_{2} / W$ alternative diffraction grating method (ADGM) (2285 $\mathrm{nm} / 715 \mathrm{~nm}$ ) stack. In figs (a) and (b), the dispersion curve has a negative slope indicating type II dispersion whereas figs. (c) and (d) shows a positive slope showing type I dispersion. $f_{T E 1}$ at $k_{x}=0$ are slightly different in each case because of the different reflector stacks.

$k_{x}=\frac{m \pi}{W}$,

where $m$ is a positive integer. The symmetric eigenmodes $(m=1,3,5 \ldots)$ were only found in the simulations because of symmetry $\left(\partial U_{z} / \partial x=0\right)$ BCs imposed. The frequencies of these modes, $f=f\left(k_{x}\right)$, determined from Comsol eigen frequency simulations were used to plot the dispersion curve. Half a structure simulations were done $(W=140 \mu \mathrm{m})$. The choice for a half structure simulation with symmetric boundary condition is convenient because it will not show the even modes that will not couple to the electrical field. Figure 4.2 shows the dispersion curves obtained from eigen frequency simulations for resonators with shear-optimized stacks designed with the (a) stop-band theory, (b) phase error approach, (c) diffraction grating method (DGM) and (d) alternative diffraction grating method (ADGM). In figures (a) and (b), the dispersion curve has a negative slope indicating type II dispersion, whereas figures (b) and (d) show a positive slope, hence type I dispersion.

The dispersion response was also confirmed from the impedance curves obtained from the combination of the Mason model and the transmission line model (see also chapter 2) [3]. Following [3],[16], the impedance curves of longitudinal waves were obtained from this model and those for the shear waves were obtained by replacing longitudinal 
wave material parameters with those of shear waves in the same model. The shear wave impedance curve has no electrical relevance, but the resonances in the curve show the frequencies at which shear mechanical resonances occur [3] and hence give a good insight of the dispersion response.

Figure 4.3 shows the impedance curves for longitudinal and shear waves of the resonators with shear-optimized stacks designed using the (a) stopband theory, (b) phase error approach, (c) diffraction grating method (DGM) and (d) alternative diffraction grating method (ADGM). In figures (a) and (b) the second shear harmonic resonance (TS2) is above the longitudinal main resonance (TE1) indicating a type II dispersion $\left(f_{\mathrm{TE} 1}<f_{\mathrm{TS} 2}\right)$ whereas in figs. $(\mathrm{c})$ and $(\mathrm{d}), f_{\mathrm{TE} 1}>f_{\mathrm{TS} 2}$ confirming type I dispersion.

In summary, we have seen that the dispersion response obtained from the analytical model is in-line with FEM simulations. The optimized stacks in which the stop-band theory and the phase error approach are adopted show a type II dispersion, while the DGM and ADGM methods seem to yield type I dispersion. The reason for this will be discussed in the next section. 


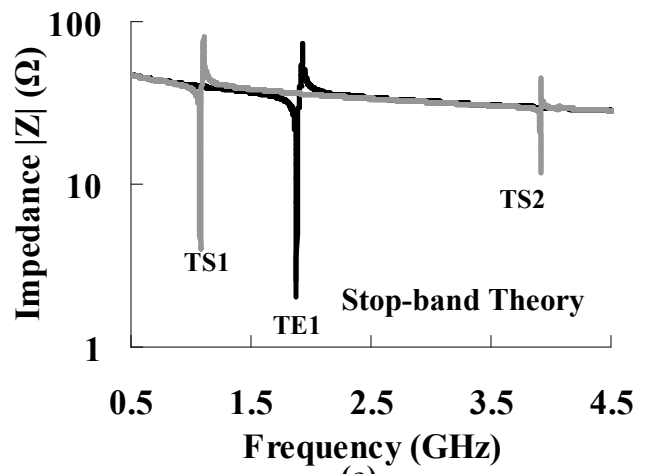

(a)

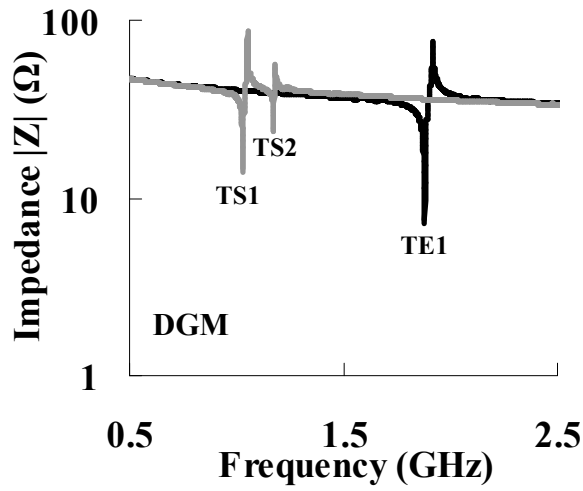

(c)

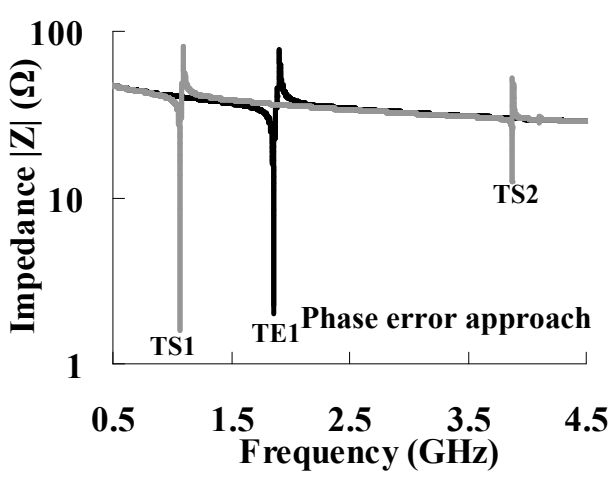

(b)

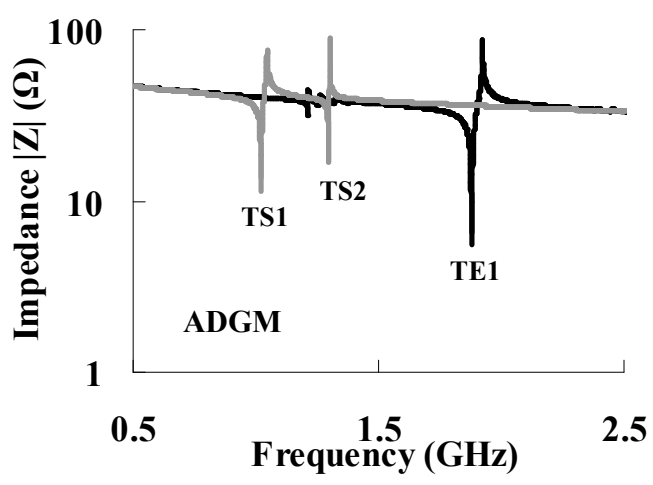

(d)

Figure 4.3: $\quad$ Modelled impedance curves for longitudinal (black) and shear (grey) waves of the resonators obtained with nine-layer $\mathrm{SiO}_{2} / \mathrm{Ta}_{2} \mathrm{O}_{5}$ shear-optimized stacks at $\approx 1.88 \mathrm{GHz}$ using (a) the stopband theory including spacer (559 $\mathrm{nm} / 862 \mathrm{~nm}$ ) (b) the phase error approach $(617 \mathrm{~nm} / 810 \mathrm{~nm})$ (c) the diffraction grating method (DGM) $(2285 \mathrm{~nm} / 1765 \mathrm{~nm})$ and (d) a five layer $\mathrm{SiO}_{2} / \mathrm{W}$ alternative diffraction grating method (ADGM) (2285 $\mathrm{nm} / 715 \mathrm{~nm})$ stack. In figs (a) and (b) the second shear harmonic resonance (TS2) is above the longitudinal main resonance (TE1) indicating a type II dispersion ( $\left.f_{T E 1}<f_{T S 2}\right)$ whereas in figs. (c) and (d), $f_{T E 1}>f_{T S 2}$ confirming type I dispersion. Active device area is $140 \times 140 \mu^{2}$.

\subsection{Flipping of the dispersion relation in SMRs}

It is obvious from section 4.1 that the reflector stack design has a significant influence on the dispersion relation of the resonators. The SMRs with a reflector stack designed with the stop-band theory and the phase error approach resulted in dispersion type II where as the ones designed with the DGM methods ended up in dispersion type I. As mentioned before, in order to use a frame region for spurious suppression, it is essential that the dispersion relation should be type I. This calls for the necessity of flipping the dispersion in the stop-band theory and phase error approach based resonators.

The proposal of increasing the thickness of the low-impedance top reflector layer (usually the oxide layer) in $\mathrm{SiO}_{2} / \mathrm{W}$ stacks for flipping the dispersion relation in $\mathrm{AlN}$ based SMRs was first reported by [2], [3]. It was then an observation that by choosing the top-oxide layer thickness large enough one arrives at the desired dispersion type I. 

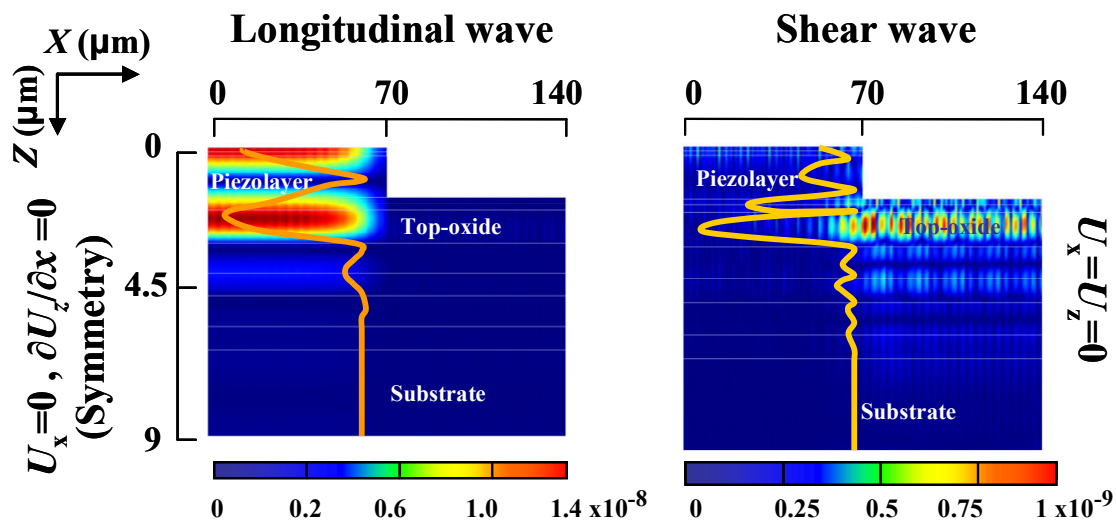

Figure 4.4 :

2D FEM results for vertical displacement of longitudinal waves (left) and shear waves (right) in an SMR with a five-layer phase error approach designed $\mathrm{SiO}_{2} / W(663 \mathrm{~nm} / 880 \mathrm{~nm})$ reflector stack optimized at $\approx 1.97 \mathrm{GHz}$ with an increased top-oxide layer of $950 \mathrm{~nm}$. The plane of symmetry is defined at the left edge of the device. Shear motion is mostly confined in the top-oxide. Boundary conditions at bottom and top are assumed to be free. The displacement profile at $x=0$ of the longitudinal and shear waves inside the device is shown in golden colour.

The possible reason for this phenomenon is explained as follows. The shear wave velocity in AIN layer is higher (around 1.5 times) than that in the oxide layer. For the generated shear wave to resonate in the AlN layer in the first order (TS1), the AlN layer needs to be thicker. The next layer which is sufficiently thick for the first order shear to resonate is the top-oxide layer provided that its thickness can be tuned accordingly. Hence when the top-oxide layer thickness is increased, the position of the longitudinal resonance $\left(f_{\text {TE1 }}\right)$ remains almost the same while the position of the shear resonances $\left(f_{\text {TS1 }}\right.$ and $\left.f_{\mathrm{TS} 2}\right)$ drop fulfilling the condition $f_{\mathrm{TE1}}>f_{\mathrm{TS} 2}$ [2]. The diffraction grating methods tend to yield thick top oxide layers which is the reason why these resonators inherently exhibit type I dispersion. The stacks optimized by these methods allow the direct application of the frame region.

The flipping of the dispersion curve from type II to type I was further studied with the aid of 2D FEM simulations in $\mathrm{SiO}_{2} / \mathrm{W}(663 \mathrm{~nm} / 880 \mathrm{~nm})$ stacks obtained with the phase error approach. The boundary conditions given in Figure 4.1 were used. Figure 4.4 shows the simulated displacement profile of longitudinal and shear waves of the resonator when the top-oxide is increased. The figure shows that the shear motion is confined in the top-oxide layer when designed to be just between type I and type II dispersion. The dispersion type was also confirmed by eigen frequency simulations and impedance curves shown in Figure 4.5 and Figure 4.6 respectively. The top-oxide layer thickness was increased to $950 \mathrm{~nm}$ to just achieve type I dispersion. The confinement of shear in the top-oxide layer motivated us to study the criteria for flipping.

Assuming that the shear component of the eigen mode is confined to the top-oxide layer, i.e. when half-wavelength fits into the oxide, the thickness of the oxide for which flipping occurs can be calculated according to:

$$
t_{\mathrm{ox}}=\frac{v_{\mathrm{S} . \mathrm{ox}}}{2 \cdot f_{\mathrm{R}}}
$$




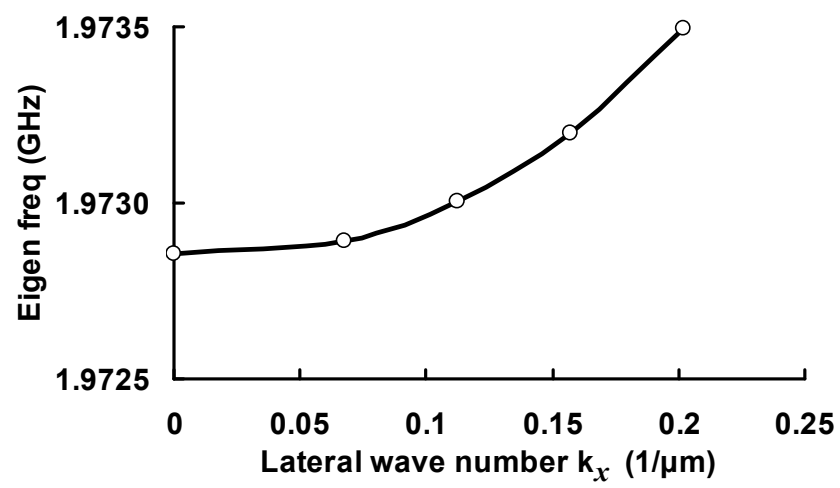

Figure 4.5:

The dispersion curve obtained from 2D FEM eigen frequency simulations for the $\mathrm{SMR}$ with a five layer $\mathrm{SiO}_{2} / \mathrm{W}$ stack $(663 \mathrm{~nm} / 880 \mathrm{~nm})$ with increased top-oxide layer thickness of $950 \mathrm{~nm}$ optimized at $\approx 1.97 \mathrm{GHz}$ using phase error approach. The curve shows a positive slope yielding type I dispersion.

with $t_{\mathrm{ox}}$ is the thickness of the top-oxide layer, $v_{\mathrm{S} \text {.ox }}$ is the shear velocity in oxide and $f_{\mathrm{R}}$ is the resonant frequency. From eq. (4.3), it can be seen that $f_{R}$ equals the resonance frequency in the oxide, $f_{\mathrm{TS} 1 \text { oxide, }}$ i.e. $f_{\mathrm{R}}=f_{\mathrm{TE} 1}=f_{\mathrm{TS} 1 \text { oxide }}$.

We further explored the dispersion condition $\left(f_{\mathrm{TE} 1}=f_{\mathrm{TS} 2}\right)$, to study the influence of the top-oxide layer below the piezoelectric layer. Neglecting the longitudinal and shear wave extension into other layers, thus with a simplified assumption that the confinement of longitudinal motion to be only in piezoelectric layer and shear motion only in the top-oxide, a criteria for flipping is derived based on: $f_{\mathrm{TE} 1}=f_{\mathrm{TS} 1 \mathrm{ox}}$, where $f_{\mathrm{TE} 1}$ is the longitudinal resonant frequency in the piezolayer and $f_{\text {TS1ox }}$ is the shear resonant frequency in the top-oxide layer. In addition, following the dispersion condition in the single piezoelectric layer: $f_{\mathrm{TE} 1}=f_{\mathrm{TS} 2}$, the flipping occurs when the full shear wavelength

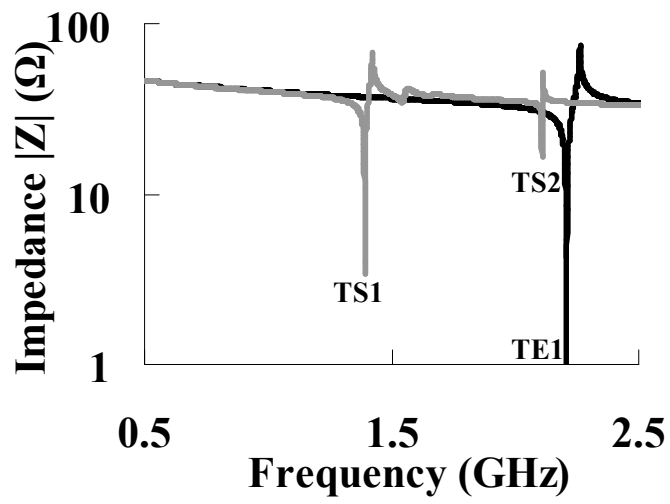

Figure 4.6:

The impedance curves for the SMR with a five layer $\mathrm{SiO}_{2} / \mathrm{W}(663 \mathrm{~nm} / 880 \mathrm{~nm})$ stack designed using phase error approach with increased top-oxide layer thickness of 950nm. The longitudinal (black) and shear (grey) curves are obtained using longitudinal and shear wave material parameters for shearoptimized stacks at $\approx 1.97 \mathrm{GHz} f_{T E 1}>f_{T S 2}$, confirming type I dispersion. Active device area is $140 \times 140 \mu^{2}$. 


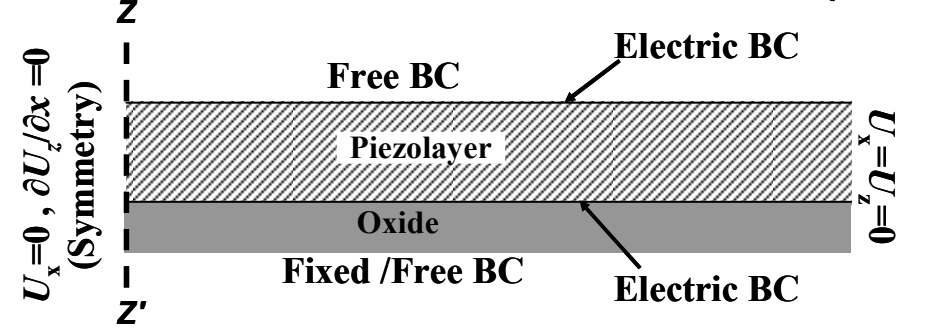

Figure 4.7: Schematic cross section of the piezoelectric layer/oxide bi-layer indicating the boundary conditions (BC). The axis of symmetry assumed in the 2D FEM simulations is indicated by a $Z-Z^{\prime}$ line. Electric boundary conditions (electric potential and ground) were applied at the bottom and top of the piezoelectric layer without using physical electrodes.

matches the piezoelectric layer thickness $\left(t_{\text {piezo }}\right)$ as well, hence when $f_{\text {TS1ox }} \approx f_{\text {TS2. }}$.

Working out the condition $f_{\mathrm{TE} 1}=f_{\mathrm{TS1}}$ further to distinguish the dispersion type I $\left(f_{\text {TE1 }}>f_{\text {TS1ox }}\right)$ from type II, gives a rule of thumb for flipping. For type I holds:

$$
\frac{v_{\text {L.piezo }}}{v_{\text {S.ox }}}>\frac{t_{\text {piezo }}}{t_{\mathrm{ox}}},
$$

\begin{tabular}{ccc}
\hline \hline (a) $\mathrm{t}_{\text {piezo }}=1500 \mathrm{~nm}$ & \\
\hline \hline$t_{\text {ox }}(\mathrm{nm})$ & $t_{\text {piezo }} / t_{\text {ox }}$ & Dispersion \\
\hline 0 & - & Type II \\
200 & 7.5 & Type II \\
300 & 5 & Type II \\
400 & 3.75 & Type II \\
$\mathbf{5 0 0}$ & $\mathbf{3}$ & Type I \\
$\mathbf{6 0 0}$ & $\mathbf{2 . 5}$ & Type I \\
$\mathbf{7 0 0}$ & $\mathbf{2 . 1 4}$ & Type I \\
$\mathbf{8 0 0}$ & $\mathbf{1 . 8 7 5}$ & Type I \\
\hline \hline (b) $t_{\text {ox }}=300 \mathrm{~nm}$ & & \\
\hline \hline$t_{\text {piezo }}(\mathrm{nm})$ & $t_{\text {piezo }} / t_{\mathrm{ox}}$ & Dispersion \\
\hline \hline 500 & $\mathbf{1 . 6}$ & Type I \\
$\mathbf{8 0 0}$ & $\mathbf{2 . 6}$ & Type I \\
$\mathbf{9 0 0}$ & $\mathbf{3 . 0}$ & Type I \\
1000 & 3.3 & Type II \\
1200 & 4.0 & Type II \\
1500 & 5.0 & Type II \\
1700 & 5.6 & Type II \\
\hline \hline
\end{tabular}

Table 4.1 Flipping of the dispersion curves observed with FEM simulations for (a) $t_{\mathrm{piez}}=1500$ nm and variable $t_{0 \mathrm{x}}$ and (b) $t_{0 \mathrm{x}}=300 \mathrm{~nm}$ and variable $t_{\text {piezo }}$. Flipping takes place at $t_{\text {piezd }} t_{0 \mathrm{x}} \approx v_{\mathrm{L}, \text { piez }} v_{\mathrm{S}, \mathrm{ox}}=2.925$. Fixed boundary condition was assumed at the bottom of the oxide layer, more or less applicable for SMRs with a quarter wavelength mirror stack. 
$v_{\text {L.piezo }}$ is the longitudinal wave velocity in the piezolayer, $v_{\text {s.ox }}$ is the shear wave velocity in the oxide layer, $t_{\text {piezo }}$ is the thickness of the piezolayer and $t_{\mathrm{ox}}$ is the thickness of the oxide layer. For type II dispersion, of course the opposite of eq. (4.4) holds ( $\left.f_{\text {TE1 }}<f_{\text {TS1ox }}\right)$.

This rule of thumb assumes longitudinal confinement in the piezoelectric layer, therefore it is essential that below the resonator, a good reflector is present for longitudinal wave as demonstrated in [2], [8]. To test the rule of thumb for a highly idealized situation, the validity of the rule is demonstrated with 2D FEM simulations of a resonator without physical electrodes and just oxide below it, as shown in Figure 4.7. The results are summarized in Table 4.1. The rule was verified for two cases: a fixed piezoelectric layer thickness with a varying oxide thickness and a fixed oxide layer thickness with a varying piezoelectric layer thickness. A bi-layer of AlN and $\mathrm{SiO}_{2}$ $\left(v_{\text {L.piezo }} / v_{\text {S.ox }}=2.925\right)$ was simulated with a fixed boundary condition ${ }^{*}$ at the bottom, more or less applicable for SMRs with a quarter wavelength mirror stack. Indeed, the results show that flipping occurs at $t_{\text {piezo }} / t_{\text {ox }} \approx v_{\text {L.piezo }} / v_{\text {S.ox. }}$

Figure 4.8 shows examples of dispersion curves obtained from FEM simulations confirming the dispersion types, positive slope representing type I and negative slope representing type II, respectively. In the case of using the physical electrodes, a part of the waves is also in the electrodes and hence flipping occurs beyond this criterion $\left(t_{\text {piezo }} / t_{\text {ox }}>v_{\text {L.piezo }} / v_{\text {S.ox }}\right)$. Figure 4.9 shows a plot of oxide thickness vs. $f_{\text {TE1 }}, f_{\text {TS2 }}$ and $f_{\text {TS1oxide }}$ showing the point of flipping. The flipping of dispersion occurs for the oxide thickness at which the three frequencies coincide. For this idealized situation, the rule of thumb works flawless. In the following discussion, the rule of thumb is verified for more realistic situations.

\footnotetext{
* The longitudinal mode shape has a node of zero displacement at the bottom of the oxide layer in the case for a quarter wave stack where the layer interfaces in the reflector are nodes of zero force and zero displacement, alternately. When the layer thicknesses deviate from quarter wave, the nodes of zero force/ displacement shift to somewhere in the middle of the layer.
} 


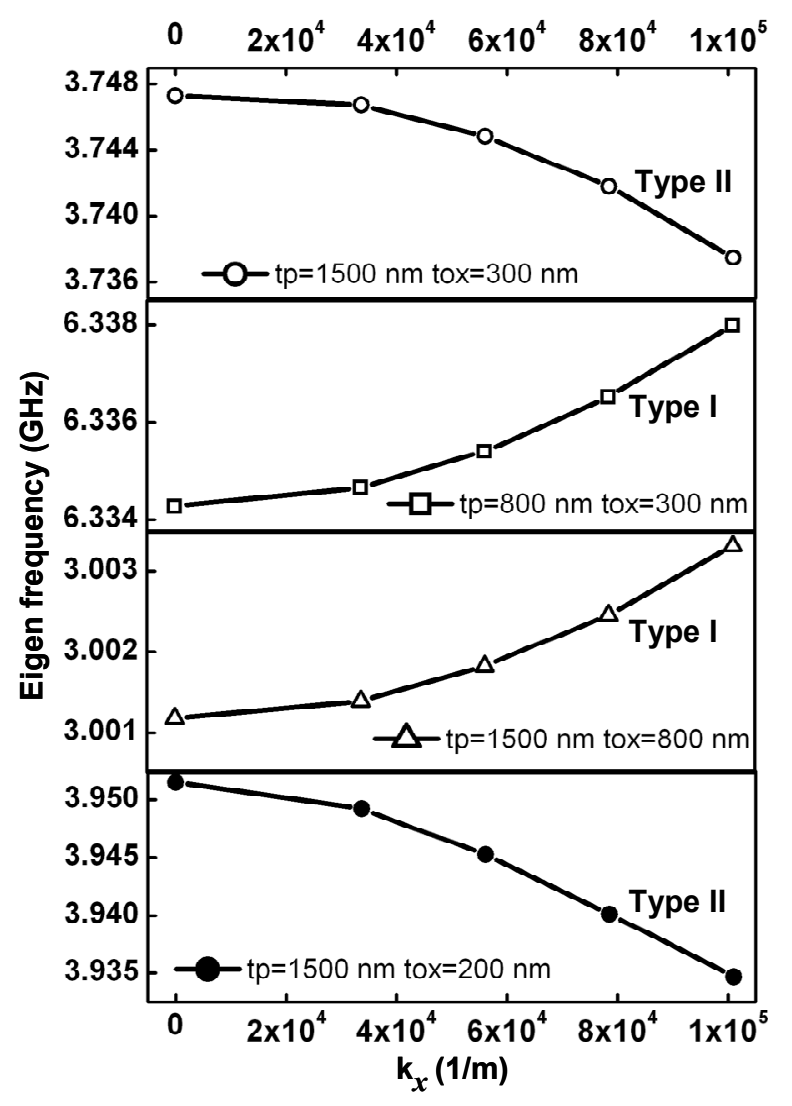

Figure 4.8: Dispersion curves obtained from 2D FEM eigen frequency simulations for the examples cases of type I (positive slope) and type II (negative slope) for both (a) and (b) (see Table 4.1). A fixed-bottom boundary condition was assumed here, more or less applicable for SMRs with a quarter wavelength mirror stack.

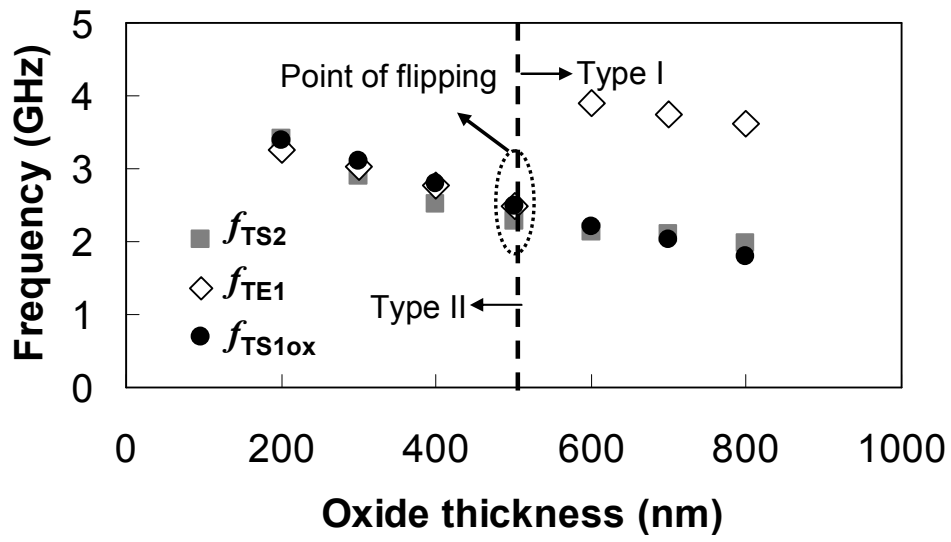

Figure 4.9: $\quad$ A plot of oxide thickness $v f_{T E 1}, f_{T S 2}$ and $f_{T S 1 o x i d e}$ showing the point of flipping. The flipping of dispersion occurs for the oxide thickness at which the three frequencies coincide. 
The flipping rule works well for SMRs with high impedance contrast (12, ref. table 3.5) $\mathrm{SiO}_{2} / \mathrm{W}$ reflector stack as demonstrated earlier in this section. However, when the rule of thumb was applied to a low impedance contrast (2.5, ref. table 3.5) $\mathrm{SiO}_{2} / \mathrm{Ta}_{2} \mathrm{O}_{5}$ reflector stack, the top-oxide layer thickness needed to be increased well beyond the piezoelectric layer thickness $(1500 \mathrm{~nm})$ for the flipping to occur. This is not a favorable situation as most of the longitudinal energy then leaks to the thick top-oxide layer, rather than confining in the piezoelectric layer itself.

A straightforward solution for this is to use a patterned metal as the topmost high acoustic impedance layer, thus combining the important advantages of a fully dielectric and a patterned metal reflector stack [4], [5]. Metals usually have high acoustic impedances; hence a high impedance contrast can be achieved (ref. Table 3.5) whereas dielectric reflectors are convenient avoiding the patterning for isolation between the resonators. The presence of half wave longitudinal resonance in the top-oxide layer was demonstrated in [17]. Although the piezoelectric layer causes the acoustical wave in an SMR, the longitudinal wave resonates in the top-oxide layer at a frequency which is different from that of the TE1 resonance because of the different longitudinal wave velocity and thickness of the oxide layer. Since the layers adjacent to the oxide used here were both stiff and heavy, almost half the wavelength is confined in the oxide layer only. From the experiment reported in [17], correspondingly the same conclusion can be drawn with respect to the shear wave confinement: if a heavy metal is used as the topmost high acoustic impedance layer in the reflector stack, the top-oxide layer can be adjusted such that shear wave finds a resonance inside the top-oxide layer.

The dispersion type of the resonators based on the stop-band theory and phase error approach was made type I by including Platinum $(\mathrm{Pt})$ as the topmost high impedance layer. In the case of stop-band theory stacks with spacer (559 nm/862 nm), two extra layers were added: a Pt layer of $280 \mathrm{~nm}$ as the high impedance layer and increased topoxide layer thickness of $980 \mathrm{~nm}$ optimized at $1.88 \mathrm{GHz}$ given by the rule of thumb. The extra layers were added in order not to disrupt the spacer configuration of the stack.

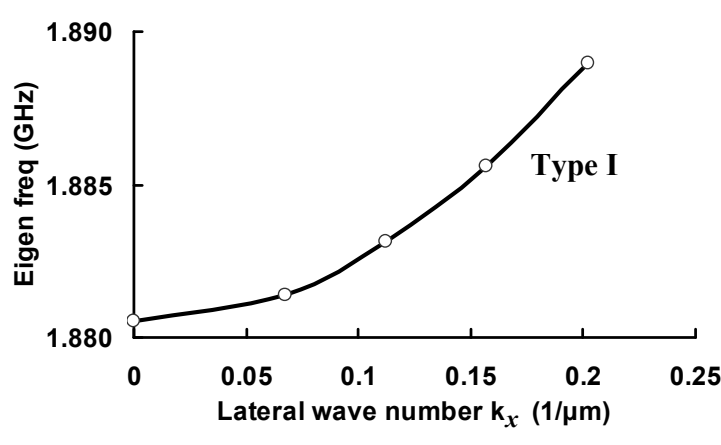

(a)

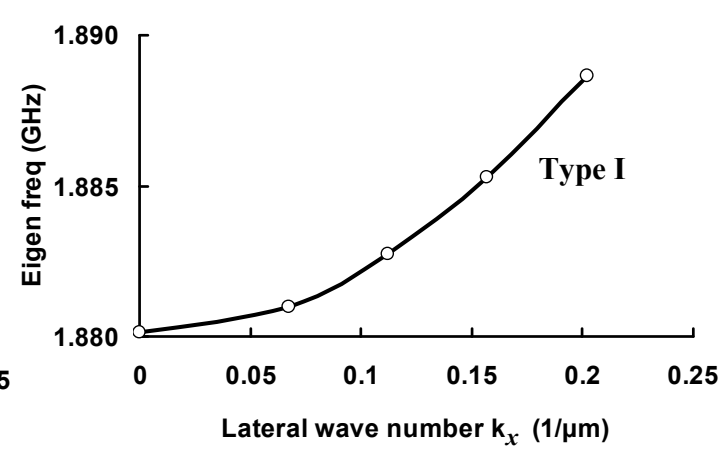

(b)

Figure 4.10:

Dispersion curves obtained from $2 D$ FEM eigen frequency simulations for resonators with a nine layer $\mathrm{SiO}_{2} / \mathrm{Ta}_{2} \mathrm{O}_{5}$ optimized stack at $\approx 1.88 \mathrm{GHz}$ obtained with (a) stopband theory with spacer $(559 \mathrm{~nm} / 862 \mathrm{~nm})$ and (b) phase error approach $(617 \mathrm{~nm} / 810 \mathrm{~nm})$ both with an increased top-oxide layer thickness of $980 \mathrm{~nm}$. A Pt layer of $280 \mathrm{~nm}$ was used as the topmost high impedance layer. The curve shows a positive slope yielding type I dispersion. Compare with figures 4.2 (a) and (b). 


\begin{tabular}{|c|c|c|}
\hline \multicolumn{3}{|c|}{ (a) tpiezo $=1500 \mathrm{~nm}$} \\
\hline$t_{\mathrm{ox}}(\mathrm{nm})$ & $t_{\text {piezo }} / t_{\mathrm{ox}}$ & Dispersion \\
\hline 0 & - & Type II \\
\hline 200 & 7.5 & Type II \\
\hline 300 & 5 & Type II \\
\hline 400 & 3.75 & Type I \\
\hline 500 & 3 & Type I \\
\hline 600 & 2.5 & Type I \\
\hline 700 & 2.14 & Type I \\
\hline 800 & 1.875 & Type I \\
\hline \multicolumn{3}{|c|}{ (b) $t_{\mathrm{ox}}=300 \mathrm{~nm}$} \\
\hline$t_{\text {piezo }}(\mathrm{nm})$ & $t_{\text {piezo }} / t_{\mathrm{ox}}$ & Dispersion \\
\hline 500 & 1.6 & Type I \\
\hline 800 & 2.6 & Type I \\
\hline 900 & 3.0 & Type I \\
\hline 1000 & 3.3 & Type I \\
\hline 1200 & 4.0 & Type II \\
\hline 1500 & 5.0 & Type II \\
\hline 1700 & 5.6 & Type II \\
\hline
\end{tabular}

Table 4.2 Flipping of the dispersion curves observed from FEM simulations for (a) $t_{\text {piezo }}=1500$ nm and variable $t_{0 \times}$ and (b) $t_{0 \mathrm{x}}=300 \mathrm{~nm}$ and variable $t_{\text {piezo. }}$ Flipping takes place when $t_{\text {piezo }} t_{0 \mathrm{x}}>v_{\mathrm{L}, \mathrm{pizz}} / v_{\mathrm{S}, \mathrm{ox}}=2.925$. Free bottom boundary condition was assumed here, more or less applicable for FBARs.

For the phase error approach designed stacks $\left(617 \mathrm{~nm} \mathrm{Ta} \mathrm{O}_{5} / 810 \mathrm{~nm} \mathrm{SiO}\right.$ ), the two upper layers of the stack were replaced by a Pt layer of $280 \mathrm{~nm}$ as the high impedance layer and an increased top-oxide layer of $980 \mathrm{~nm}$ optimized at $1.88 \mathrm{GHz}$. When increasing the top-oxide layer thickness for the stacks optimized at a particular resonant frequency $\left(f_{R}\right)$, there can be a small variation in $f_{R}[8] . f_{R}$ can be readjusted by either the top-electrode thickness and/or the piezo layer thickness. The dispersion curves obtained from 2D FEM eigen frequency simulations of the SMRs with improved stacks for type I dispersion are shown in Figure 4.10. This confirms that the rule of thumb works for SMRs with a complete reflector stack used in practical situations.

\subsection{Flipping of the dispersion curve extended to FBARs}

The exercises were repeated for the same $\mathrm{AlN} / \mathrm{SiO}_{2}$ bi-layer with a free boundary condition (BC) at the bottom, the BC applicable for FBARs (see Figure 4.7). The flipping was also observed with free bottom BC indicating the rule of thumb can also be applied for FBARs. The results are summarized in Table 4.2. Also here, the rule was verified for two cases: a fixed piezoelectric layer thickness with varying oxide thickness and a fixed oxide layer thickness with varying piezoelectric layer thickness. The flipping again occurs when $t_{\text {piezo }} / t_{\text {ox }}>v_{\text {L.piezo }} / v_{\text {S.ox. }}$ Figure 4.11 shows examples of dispersion curves obtained from FEM simulations confirming the dispersion types. 


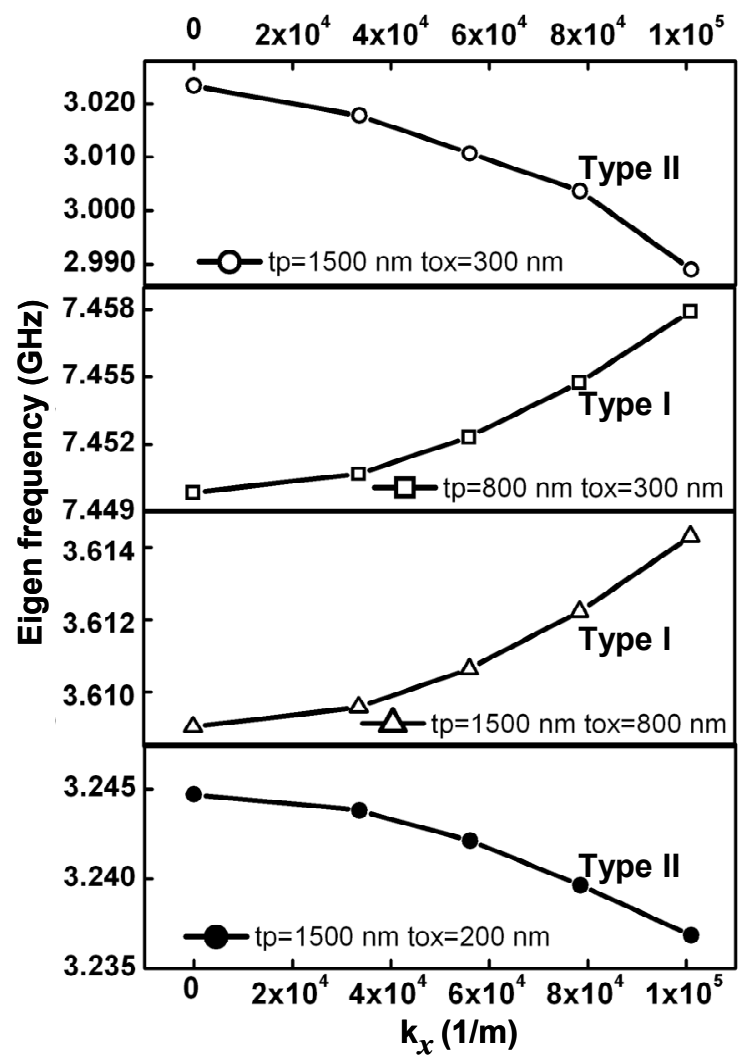

Figure 4.11: Dispersion curves obtained from 2D FEM Eigen frequency simulations for the example cases of type I (positive slope) and type II (negative slope) for both (a) and (b) (see Table 4.2). Free bottom boundary condition was assumed here, more or less applicable to FBARs.

The influence of the shear wave velocity in the oxide, $v_{\text {S.ox }}$ on the dispersion type was also examined by varying the shear velocity of the oxide layer in 1D Mason model. The simulations were done on a bi-layer with $t_{\text {piezo }}=1500 \mathrm{~nm}$ and $t_{\mathrm{ox}}=300 \mathrm{~nm}$ as shown in Table 4.3. It was observed that the dispersion type was just between type I and II dispersion $\left(f_{\mathrm{TE1}}=f_{\mathrm{TS} 1 \mathrm{ox}} \approx f_{\mathrm{TS} 2}\right)$, when $v_{\text {L.piezo }} / v_{\mathrm{S} . \mathrm{ox}} \approx 4$ and $v_{\mathrm{S} \text {.piezo }} / v_{\mathrm{S} . \mathrm{ox}} \approx 2$.

\begin{tabular}{cc}
\hline \hline$V_{\text {s.ox }}$ & Dispersion \\
\hline 4351 & Type II \\
3351 & Type II \\
3051 & Type II \\
2751 & $\boldsymbol{f}_{\text {TE1 }}=\boldsymbol{f}_{\text {TS1ox }}$ \\
2651 & Type I \\
2451 & Type I \\
2351 & Type I \\
\hline \hline
\end{tabular}

Table 4.3:

Influence of shear wave velocity in the oxide $v_{\mathrm{S} .0 \mathrm{x}}$ on the dispersion type. $A$ bilayer with $t_{\mathrm{piez}}=1500 \mathrm{~nm}$ and $t_{\mathrm{ox}}=300 \mathrm{~nm}$ was assumed in the $1 \mathrm{D}$ Mason model. $f_{\mathrm{TE} 1} \approx 3.2 \mathrm{GHz}$. Free bottom boundary condition was assumed here, more or less applicable to FBARs. 


\subsection{Discussion}

The rule of thumb for flipping the dispersion is derived on the basis of few assumptions. This section covers some guidelines in judging the applicability of the proposed method. Most importantly, as mentioned before the rule is derived assuming the confinement of longitudinal motion only in piezoelectric layer and shear motion only in the top-oxide, thus neglecting the wave extension into other layers. In reality, the waves slightly extend into other layers in the stack. Therefore, it is advisable to include all the layers including the bottom electrodes in the Mason transmission line model.

It is to be noted that when TE1 and TS2 modes are very close to each other in frequency, the dispersion type becomes undetermined which is unique to the SMR [3],[18]. Hence in practical cases, starting from eq.(4.3), it is recommended to sweep the top-oxide layer thickness a few nanometers (for e.g. in steps of $25 \mathrm{~nm} \mathrm{[2])} \mathrm{until} \mathrm{the} \mathrm{dispersion} \mathrm{type}$ becomes unambiguous.

Also the flipping scenario discussed in this chapter is mainly based on AlN as the piezoelectric layer and $\mathrm{SiO}_{2}$ as the adjacent layer. We examined the adaptability of the rule for other materials as well. The rule applies for the bi-layer combination of AlN and Aluminium as mentioned earlier [2].

Further, the rule is not applicable for flipping type I materials to type II because its shear confinement is not only in the top-oxide layer but spreads over the whole bi-layer. However, flipping is observed in a bi-layer of $\mathrm{ZnO}$ (type I material) with $\mathrm{Pt}$ but this is not yet understood. The FEM simulation results for a $\mathrm{ZnO}$ based resonator of $1500 \mathrm{~nm}$ are summarized in Table 4.4 .

In addition, the bi-layer examples shown with fixed and free boundary conditions are without physical electrodes. With the physical electrodes, parts of the waves are also in the electrodes (in FBARs), and also in other layers (in SMRs). Hence flipping occurs beyond the rule $\left(t_{\text {piezo }} / t_{\mathrm{ox}}>\mathrm{v}_{\mathrm{L} \text {,piezo }} / \mathrm{v}_{\mathrm{S}, \mathrm{ox}}\right)$ in practical situations. The rule assumes

\begin{tabular}{cccc}
\hline \hline$t_{\mathrm{Pt}}(\mathrm{nm})$ & $t_{\text {piezo }} / t_{\mathrm{Pt}}$ & $\begin{array}{c}\text { Fixed BC: } \\
\text { Dispersion }\end{array}$ & $\begin{array}{c}\text { Free BC }: \\
\text { Dispersion }\end{array}$ \\
\hline 0 & - & Type I & Type I \\
200 & 7.5 & Type II & Type II \\
300 & 5 & Type II & Type II \\
400 & 3.75 & Type II & Type II \\
500 & 3 & Type II & Type II \\
600 & 2.5 & Type II & Type I \\
700 & 2.14 & Type I & Type I \\
800 & 1.875 & Type I & Type I \\
\hline \hline
\end{tabular}

Table 4.4 Flipping of the dispersion curves for a bi-layer of $\mathrm{ZnO}$ and Pt (a) $t_{\text {piezo }}=1500 \mathrm{~nm}$ and variable $t_{\mathrm{Pt}}$. The flipping takes place around $t_{\mathrm{piezo}} / t_{\mathrm{Pt}}>v_{\mathrm{L}, \text { piezo }} / v_{\mathrm{S}, \mathrm{Pt}}=3.53$. Fixed and free bottom boundary conditions were assumed here, more or less applicable for SMRs and FBARs respectively. 
longitudinal confinement in the piezoelectric layer. It is therefore essential that the reflector stack should be a good reflector for the longitudinal wave. This could be the reason why the rule immediately applies for SMRs employing high impedance contrast $\mathrm{SiO}_{2} / \mathrm{W}$ stacks compared to low impedance contrast $\mathrm{SiO}_{2} / \mathrm{Ta}_{2} \mathrm{O}_{5}$ stacks. The rule can be implemented in low impedance contrast stack by using a metal as the topmost high acoustic impedance layer.

The rule of thumb was applied to various bi-layers with $\mathrm{AlN}$ and $\mathrm{ZnO}$ as the piezoelectric layers in combination with $\mathrm{SiO}_{2}$, Aluminium and Platinum as the adjascent layers. For the bi-layer combinations for which the rule of thumb works, we observed the ratio of the longitudinal velocity in the piezoelectric layer to that of shear wave velocity in the adjacent layer was found to be around $3\left(\mathrm{AlN} / \mathrm{SiO}_{2}: 3.16, \mathrm{AlN} / \mathrm{Al}: 3.55\right.$ and $\mathrm{ZnO} / \mathrm{Pt}$ : 3.53).

The influence of the shear wave velocity in the adjacent layer on the dispersion type was also considered in the study (see Table 4.3 ) and it was observed that the dispersion type was just between type I and II dispersion when $v_{\text {L.piezo }} / v_{\text {S.ox }} \approx 4$ and $v_{\text {S.piezo }} / v_{\text {S.ox }} \approx 2$. This was more or less observed in the case of $\mathrm{ZnO}$ and $\mathrm{Pt}$ as well. However, the material parameter aspect (for instance, stiffness or mass density) which is responsible for the flipping is beyond the scope of this thesis and could be a future topic of research for the material science experts.

\subsection{Conclusions}

In this chapter, the influence of the reflector stack on the acoustic dispersion of the SMRs has been addressed. It was found that the SMRs with a reflector stack obtained from the stop-band theory and the phase error approach resulted in dispersion type II where as the ones designed with the diffraction grating methods ended up in dispersion type I. In practice, the resonators should be exhibiting type I for effectively using a frame region as the spurious mode suppression scheme.

A rule of thumb for flipping dispersion curves has been proposed and verified with $2 \mathrm{D}$ FEM simulations for an SMR with a $\mathrm{SiO}_{2} / \mathrm{W}$ stack, based on the condition that the shear wave resonates in the top-oxide layer. The rule of thumb works particularly well for SMRs employing high impedance contrast (12, ref. table 3.5) stacks compared to low contrast (2.5, ref. table 3.5) ones. It can be employed in a low impedance contrast stack by using a metal as the topmost high acoustic impedance layer. The rule was verified for a bi-layer resonator containing an $\mathrm{AlN}$ layer and $\mathrm{SiO}_{2}$ as the adjacent layer with fixed bottom boundary condition, more or less emulating SMRs with a quarter wavelength mirror stack. It was observed that applying this rule and incorporating $\mathrm{Pt}$ as the topmost high impedance layer, stop-band theory and phase error approach based resonators found to be exhibiting type I dispersion.

The rule was also demonstrated for the same $\mathrm{AlN} / \mathrm{SiO}_{2}$ bi-layer with a free boundary condition at the bottom, for which the flipping was also observed indicating the rule of thumb can be applied to FBARs. However these results need to be experimentally verified. 
The rule is not applicable for flipping type I materials (e.g. ZnO) to Type II because its shear confinement is not only in the top-oxide but spreads over the whole bi-layer. However, flipping is observed in a $\mathrm{ZnO} / \mathrm{Pt}$ bi-layer, but this observation is not yet understood and could be a topic of future work.

Although the rule of thumb has been derived under specific assumptions as discussed in the chapter, it provides a sanity guide-line for the BAW designers in deciding the dispersion type of the resonator utilizing the influence of the adjacent layer in the system.

\section{References}

[1] S. Jose, R. J. E. Hueting and A. B. M. Jansman, "On the rule of thumb for flipping of the dispersion relation in BAW devices," Proc. IEEE Ultrasonics Symposium, October, 2011.

[2] G. G. Fattinger, S. Marksteiner, J. Kaitila, and R. Aigner, "Optimization of acoustic dispersion for high performance thin film BAW resonators," Proc. IEEE Ultrasonics Symposium, pp. 1175-1178, 2005.

[3] S. Marksteiner, G. G. Fattinger, R. Aigner and J. Kaitila, Acoustic Reflector for a BAW resonator providing specified reflection of both shear wave and longitudinal waves, US patent: 006933807B2., Aug. 2005.

[4] J. W. Lobeek, R. Strijbos, A. B. M. Jansman, N. X. Li, A. B .Smolders and N. Pulsford, "High-Q BAW resonator on $\mathrm{Pt} / \mathrm{Ta}_{2} \mathrm{O}_{5} / \mathrm{SiO}_{2}$-based reflector stack," Proc. IEEE Microwave Symposium, pp. 2047-2050, 2007.

[5] R. Strijbos, A. B. M. Jansman, J. W. Lobeek, N. X. Li and N. Pulsford, "Design and characterization of high- $Q$ Solidly-Mounted Bulk Acoustic Wave filters," Proc. IEEE Electronic components and technology conference, pp. 169-174, 2007.

[6] R. Ruby, "Review and comparison of Bulk Acoustic Wave FBAR, SMR technology," Proc. IEEE Ultrasonics Symposium, pp. 1029-1040, 2007.

[7] T. Pensala, R. Thalhammer, J. Dekker, and J. Kaitila, "Experimental Investigation of Acoustic Substrate Losses in 1850-MHz Thin Film BAW Resonator," IEEE Trans. on Ultrasonics, Ferroelectrics and Frequency Control, vol. 56, no.11, pp. 2544-2552, 2009.

[8] F. Thalmayr, K. Hashimoto, T. Omori and M. Yamaguchi, "Plate mode propagation losses in solidly mounted resonators," IEEE Transactions on Ultrasonics, Ferroelectrics, and Frequency Control, vol. 57, pp. 2844-2849, 2010.

[9] K. Kokkonen, T. Pensala and M. Kaivola, "Dispersion and mirror transmission characteristics of bulk acoustic wave resonators," IEEE Transactions on Ultrasonics, Ferroelectrics, and Frequency Control, vol. 58, pp. 215-225, 2011.

[10] R. Thalhammer, J. Kaitila, S. Zieglmeier and L. Elbrecht, "Spurious mode suppression in BAW resonators," Proc. IEEE Ultrasonics Symposium, pp. 456-459, 2006.

[11]G. G. Fattinger, "BAW resonators design considerations -An overview," Proc. IEEE International Frequency Control Symposium, pp. 762-767, 2008.

[12] Comsol Multiphysics, version 3.5, 2009 (www.comsol.com).

[13]T. Pensala and M. Ylilammi, "Spurious Resonance Suppression in Gigahertz-Range ZnO Thin-Film Bulk Acoustic Wave Resonators by the Boundary Frame Method: Modeling and 
Experiment," IEEE Transactions on Ultrasonics, Ferroelectrics, and Frequency Control, vol. 56, pp. 1731-1744, 2009.

[14] T. Pensala, M. Ylilammi, J. Meltaus, K. Kokkonen, " Area and Dispersion Dependence of Vibration Shape and Coupling Coefficient in Thin Film BAW Resonators," in Proc. IEEE Ultrasonics Symposium, pp. 1661-1664, 2007.

[15] A.B.M. Jansman, R. C. Strijbos, J. W. Lobeek, F. W. M. Vanhelmont, J. J. M. Ruigrok, T. Pensala, M. Ylilammi, " Application of a Vibrating Membrane Model to Bulk-AcousticWave Resonators," in Proc. IEEE Ultrasonics Symposium, pp. 1045-1048, 2007.

[16] K. M. Lakin, G. R. Kilne and K.T. McCarron, "High- $Q$ microwave acoustic resonators and filters," IEEE Transactions on Microwave Theory and Techniques, vol. 41, no. 12, pp. 21392146, 1993.

[17]J. Olivares, E. Wegmann, J.Capilla, E. Iborra, M. Clement, L. Vergara and R. Aigner, " Sputtered $\mathrm{SiO}_{2}$ as low acoustic impedance material for Bragg mirror fabrication in BAW resonators," IEEE Transactions on Ultrasonics, Ferroelectrics, and Frequency Control, vol. 57, no. 1, pp. 23-29, 2010.

[18] J. Kaitila, "Review of wave propagation in BAW thin film devices progress and prospects," Proc.IEEE Ultrasonics Symposium, pp. 120-129, 2007. 


\section{High $Q$ Solidly Mounted Resonators: Experimental Results}

This chapter deals with the experimental investigation of SMR-BAW resonators with optimized dual wave reflector stacks. These stacks were designed with the design approaches presented in chapter three and were realized with two different material combinations; one consisting of dielectrics only $\left(\mathrm{SiO}_{2} / \mathrm{Ta}_{2} \mathrm{O}_{5}\right)$ and the other of a metal-dielectric combination $\left(\mathrm{SiO}_{2} / \mathrm{W}\right)$. The improvements in the reflection of the stacks are projected on the $Q$ factor measurements from the impedance curves. 


\subsection{BAW reflector experiments}

In chapter 3, we have investigated the impact of the reflector stack design on longitudinal and shear wave transmissions. Three different design approaches were proposed. In this chapter, we seek the experimental verification of these approaches. To this purpose, BAW resonators were realized at NXP semiconductors, Eindhoven, The Netherlands and an external company. Resonators with two types of reflector stacks have been devised; one consisting of dielectrics only $\left(\mathrm{SiO}_{2} / \mathrm{Ta}_{2} \mathrm{O}_{5}\right)$ and the other of a dielectric-metal combination $\left(\mathrm{SiO}_{2} / \mathrm{W}\right)$. The realized resonators are for US-PCS (Transmit band or downlink: $1.85-1.91 \mathrm{GHz}$, Receive band or uplink: $1.93-1.99 \mathrm{GHz}$ ) applications. The resonators based on $\mathrm{SiO}_{2} / \mathrm{Ta}_{2} \mathrm{O}_{5}$ and $\mathrm{SiO}_{2} / \mathrm{W}$ stacks were optimized for the uplink and the downlink frequencies respectively.

\begin{tabular}{lccccccc}
\hline \hline Sample & $\mathbf{1}$ & $\mathbf{2}$ & $\mathbf{3}$ & $\mathbf{4}$ & $\mathbf{5}$ & $\mathbf{6}$ & $\mathbf{7}$ \\
\hline \hline \multicolumn{1}{c}{ Stack } & $\mathrm{SiO}_{2} /$ & $\mathrm{SiO}_{2} /$ & $\mathrm{SiO}_{2} /$ & $\mathrm{SiO}_{2} /$ & $\mathrm{SiO}_{2} /$ & $\mathrm{SiO}_{2} /$ & $\mathrm{SiO}_{2} /$ \\
$\mathbf{( L / H )}$ & $\mathrm{Ta}_{2} \mathrm{O}_{5}$ & $\mathrm{Ta}_{2} \mathrm{O}_{5}$ & $\mathbf{W}$ & $\mathbf{W}$ & $\mathbf{W}$ & $\mathbf{W}$ & $\mathbf{W}$ \\
\hline \hline Comment & $\mathrm{QW}$ & $\mathrm{SBT}$ & $c=0.5$ & $c=1.0$ & $c=1.5$ & $c=3.0$ & $\mathrm{DGM}$ \\
\hline $\mathbf{L}_{\mathbf{1}}(\mathbf{n m})$ & 770 & 559 & 1132 & 837 & 663 & 410 & 2240 \\
$\mathbf{H}_{\mathbf{1}}(\mathbf{n m})$ & 580 & 862 & 500 & 738 & 880 & 1085 & 660 \\
$\mathbf{L}_{\mathbf{2}}(\mathbf{n m})$ & 770 & 559 & 1132 & 837 & 663 & 410 & 2240 \\
$\mathbf{H}_{\mathbf{2}}(\mathbf{n m})$ & 580 & 431 & 500 & 738 & 880 & 1085 & 660 \\
$\mathbf{L}_{\mathbf{3}}(\mathbf{n m})$ & 770 & 1118 & 1132 & 1000 & 1000 & 1000 & 2240 \\
$\mathbf{H}_{\mathbf{3}}(\mathbf{n m})$ & 580 & 431 & - & - & - & - & - \\
$\mathbf{L}_{\mathbf{4}}(\mathbf{n m})$ & 770 & 559 & - & - & - & - & - \\
$\mathbf{H}_{\mathbf{4}}(\mathbf{n m})$ & 580 & 862 & - & - & - & - & - \\
$\mathbf{L}_{5}(\mathbf{n m})$ & 770 & 559 & - & - & - & - & - \\
\hline \hline
\end{tabular}

Table 5.1. Layer thicknesses of the reflector stack under study. Seven different samples were analyzed: two with $\mathrm{SiO}_{2} / \mathrm{Ta}_{2} \mathrm{O}_{5}$ stacks and the other five with $\mathrm{SiO}_{2} / W$ stacks. $L$ and $\mathrm{H}$ indicate lavers having low and high acoustic impedance respectively. $L_{1}$ is the first layer from the substrate. $Q W$-quarter wave stack, stop-band theory (SBT) stack, $c$ variations stacks are the phase error approach based ones and DGM-diffraction gating method based stack. Note that in sample 2, two extra layers $\mathrm{H}_{5}$ and $\mathrm{L}_{6}$ of $\mathrm{Pt}(280 \mathrm{~nm})$ and $\mathrm{SiO}_{2}(980 \mathrm{~nm})$ were added for obtaining the preferred dispersion relation (type I). In samples 4, 5 and 6 top oxide $\left(L_{3}\right)$ is increased to $1000 \mathrm{~nm}$ for flipping to type I dispersion. 


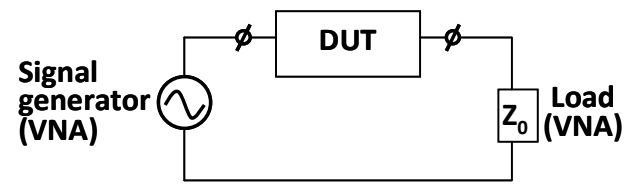

(a)

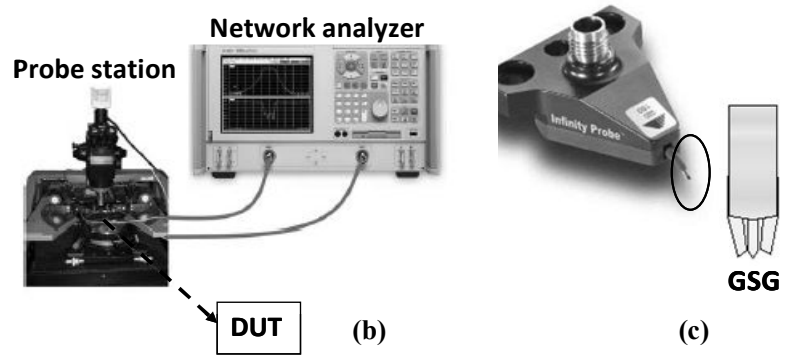

Figure 5.1: (a) Schematic representation of the measurement set-up with a microwave vector network analyzer (VNA) and Device Under Test (DUT) [3] (b) The electrical measurement set-up for electrical characterization with a VNA, RF probe station and DUT (c) coplanar RF probe with a GSG (Ground-Signal-Ground) tip.

The layer thicknesses of the reflector stacks investigated in this chapter are summarized in Table 5.1. $\mathrm{L}$ and $\mathrm{H}$ indicate layers having a low and high acoustic impedance respectively. The low impedance layer silicon oxide is deposited by plasma enhanced chemical vapour deposition (PECVD) techniques. The high impedance layers $\mathrm{Ta}_{2} \mathrm{O}_{5}$ and $\mathrm{W}$ layers were realized using ion beam deposition and sputtering respectively.

The improvements in the transmission of the stacks were analyzed from the electrical characteristics of the resonators. The measurement set-up for electrical characterization is detailed in section 5.2. The performance improvement of the resonators with the stopband theory based reflector stack is addressed in section 5.3 and the ones based on the phase error approach optimization scheme and the diffraction grating method are reviewed in section 5.4. The experiments demonstrate the credibility of the design approaches. This chapter presents the work described in [1] .

\subsection{Measurement set-up}

Thin film BAW resonators were electrically characterized by measuring their scattering parameters or S-parameters with a vector network analyzer (VNA) on wafer level with coplanar RF probes [2]. The resonator performance is typically characterized by measuring the reflection coefficient $S_{11}$ [2] of a one-port configuration.

Figure 5.1 (a) shows the schematic measurement set-up, Figure 5.1 (b) shows the actual electrical measurement set-up which includes a microwave network analyzer, a probe 


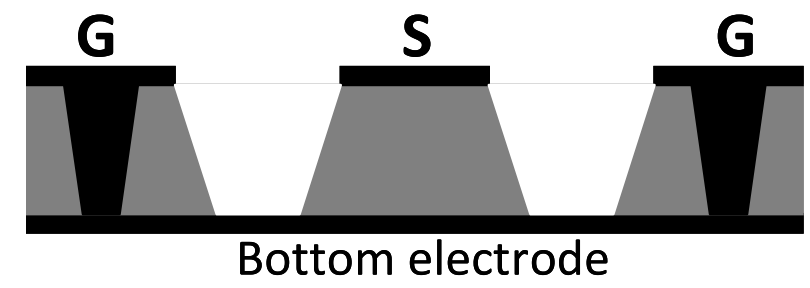

Figure 5.2: $\quad$ Schematic cross-section of the typical GSG geometry used for the 1-port electrical characterization of $B A W$ resonators. The grey shade shows the piezoelectric layer, while the darker shade indicates the bottom electrode and the top electrode patterned as GSG configuration. Note that a via through the piezolayer is needed in order to contact the ground pad.

station to connect to the resonator and the device under test (DUT), and Figure 5.1 (c) shows a coplanar RF probe with Ground-Signal-Ground (GSG) tip configuration.

Before performing any device measurement with a VNA, a calibration of reflected and transmitted power is necessary in order to circumvent systematic errors. One-port RF characterization requires calibration using thru-open-short-and-load (TOSL) calibration structures [3] provided by the microwave-instrumentation companies. By using the TOSL calibration routine standards, the VNA is calibrated till the RF probe tips thus compensating the effect of the probing system including cables and the connectors. The remaining parasitic contributions from the measurement set-up that is difficult to remove by any calibration include the contact resistance from the probes to the contact pads and the inductance of the loop formed by the electrodes from one tip to another [2], [4]. A conventional way to eliminate these contributions is to use de-embedding structures which constitute these losses, typically so-called "short" and "open" structures. The GSG (or GS) structures are carefully designed for the usage of a coplanar RF probe, so that the possible parasitics remain the same for the device variations. An example of the layout of the typical GSG geometry of a BAW resonator is shown in Figure 5.2.

The electrical responses of the SMRs were analyzed by RF measurements using a Rohde \& Schwarz ZVB20 VNA. 1-port scattering parameters $\left(S_{11}\right)$ were measured using GSG250 coplanar RF probes and impedance curves were plotted. A low power level of $20 \mathrm{dBm}$ was used to avoid any nonlinearities in the measurements.

The VNA measurement is performed within the characteristic impedance of $Z_{0}=50 \Omega$ environment which is the port impedance of the network analyzer [2]-[4]. From the measured $S_{11}$, the complex resonator impedance can be obtained by [2], [4]-[5]:

$Z=Z_{0} \cdot \frac{1+S_{11}}{1-S_{11}}$

Alternatively, some network analyzers [6] facilitate direct impedance measurement, either as the magnitude or phase of the impedance curve. The quality factor ( $Q$ factor) of 
the resonators can then be calculated from any of the methods discussed in section 2.3.2. Although the formulas for calculating the $Q$ factor are well defined, obtaining a reliable $Q$ from experiments is challenging [7]-[8]. Methods for determining the $Q$ are quite sensitive to the frequency step size in the measured range [9]. Moreover, any spurious modes or other non-idealities at the measured frequency greatly complicate a direct $Q$ calculation from the measured S-parameters [8].

It is therefore convenient to fit the measured response to the impedance obtained from the mBVD model to calculate the $Q$ [5], [8] as discussed in section 2.1.1. However, the accuracy of this approach depends on how this fitting is done [5], [8]-[9]. A quantitative comparison of $Q$ values obtained from various resonators is legitimate only when the same computation method has been employed.

\section{3 $Q$ improvement of the dielectric $\left(\mathrm{SiO}_{2} / \mathrm{Ta}_{2} \mathrm{O}_{5}\right)$ reflector stacks}

SMRs with optimized reflector stacks adopting design approaches [10] discussed in chapter 3 have been realized and characterized at NXP Semiconductors, The Netherlands. Some of the fabricated stacks [11]-[12] closely resemble the stacks proposed by the stopband theory with spacer layers (sample 2, Table 5.1). The reflector stacks used here are unique in the sense that they consist of only dielectric layers. The main advantage of using a fully dielectric stack is that the reflector stack layers need not be patterned [11]-[12]. However, since most of the dielectric combinations result in comparatively low impedance contrast stacks, a higher number of layers is needed to achieve the specified transmission.

A He-Ion cross section image of the fabricated SMR is shown in Figure 5.3. The SMR has

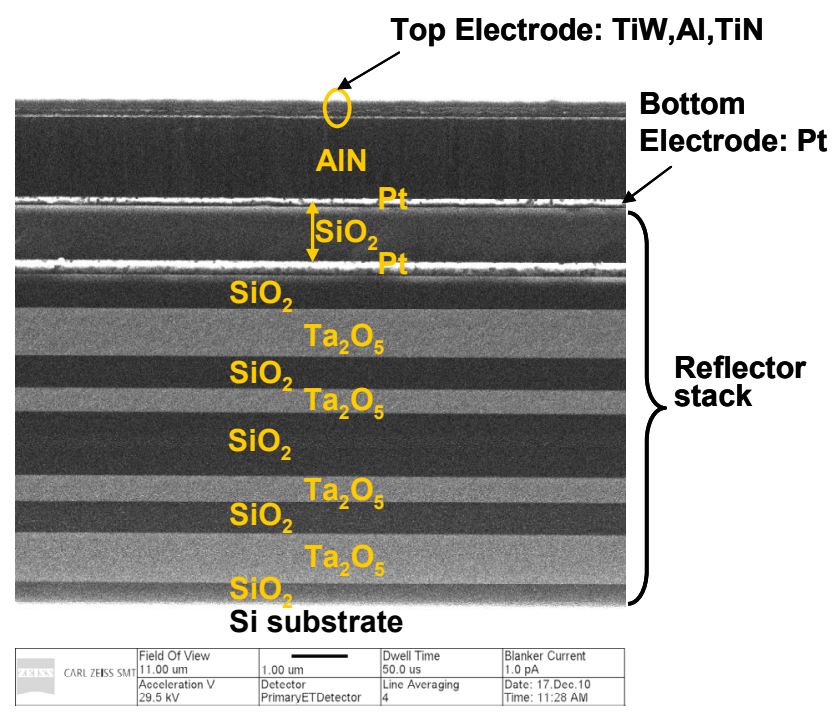

Figure 5.3: He-Ion microscope image of the cross section of an $\mathrm{SMR}$ with nine layers $\mathrm{SiO}_{2} / \mathrm{Ta}_{2} \mathrm{O}_{5}$ reflector stack [courtesy to Gregor Hlawacek and Joost Melai, MESA ${ }^{+}$institute for nanotechnology]. Note that two extra layers of $\mathrm{Pt}$ and $\mathrm{SiO}_{2}$ were added for obtaining the preferred dispersion relation (type I). 


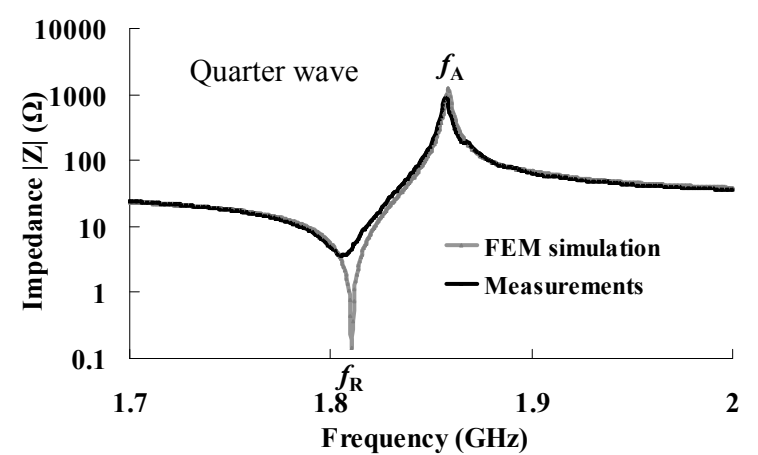

(a)

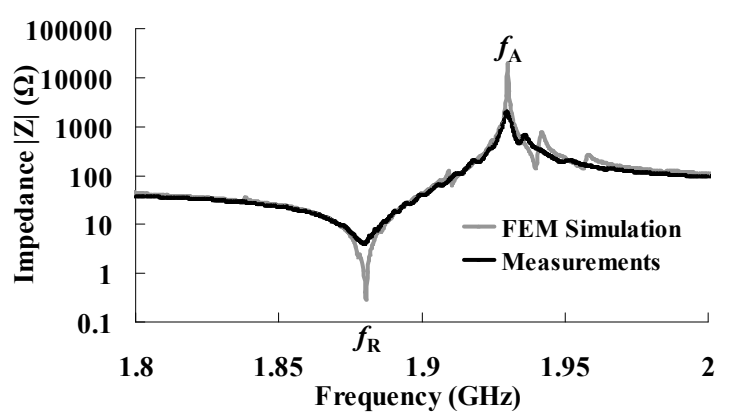

(b)

Figure 5.4: $\quad$ Comparisons between impedance curves obtained from FEM simulations and measurements of a $300 \times 100 \mu m^{2}$ SMR with (a) quarter wave reflector stack and (b) shear optimized reflector stack. The SMR with shear optimized reflector stack shows a high peak at anti-resonance in the impedance curve. The presence of spurious modes in the shear optimized impedance curve is also an indication of energy confinement.

nine layers of $\mathrm{SiO}_{2} / \mathrm{Ta}_{2} \mathrm{O}_{5}$ stacks optimized for $1.88 \mathrm{GHz}$ with Aluminum Nitride (AIN) as the piezoelectric layer. The most noticeable deviation from the stack designed is the topmost low-impedance layer for optimizing the dispersion type which was discussed in chapter 4.

A frequency step of $25 \mathrm{kHz}$ was used to extract the $Q$-factor at anti-resonance $Q_{\mathrm{A}}{ }^{\varphi}$ (see phase derivative method, eq. 2.16), from the steepness of the phase $(\varphi(f))$ curves measured directly from the analyzer [13]. Although the $Q$ values calculated by this method are sensitive to the frequency step $d f$ [14], [17] this method is used here for illustrating the efficiency of the reflector stack. Alternatively, a more robust method by fitting the electrical response using a modified Butterworth-van-Dyke (mBVD) model (section 2.2.2) has also been used for $Q$ determination for a series of resonators to extract the $1 \mathrm{D} Q$-factor.

Figure 5.4 shows a comparison between measured and 2D FEM simulated [18] impedance curves for a resonator designed with a conventional quarter-wave reflector stack and optimized dual wave reflector stack; the simulations are in good agreement with the measurements. In the SMR with a shear optimized reflector stack, the piezoelectric layer thickness was adapted to bring $f_{\mathrm{R}}$ to $1.88 \mathrm{GHz}$. For a $300 \times 100 \mu \mathrm{m}^{2}$ conventional quarter-wave reflector device a $Q_{\mathrm{A}}{ }^{\varphi}$ value of 1075 is obtained whereas the device with optimized stacks exhibits a $Q_{\mathrm{A}}{ }^{\varphi}$ of around 1940. As the $Q$-factor at resonance, $Q_{R}$, is limited by ohmic rather than acoustic losses, the improvement in $Q_{R}$ is small. Note that the shear optimized mirror increased the maximum impedance at antiresonance by more than a factor of 2 compared to the quarter-wave stack.

Furthermore, impedance plots of the device with the shear optimized reflector stack obtained from FEM simulations and measurements, shown in Figure 5.4, show spurious resonance peaks besides the main peak. The appearance of spurs is a sign of acoustic energy confinement. Although this is an undesired side effect, this is an indication that the acoustic energy is better confined in the new reflector stack than in the conventional 


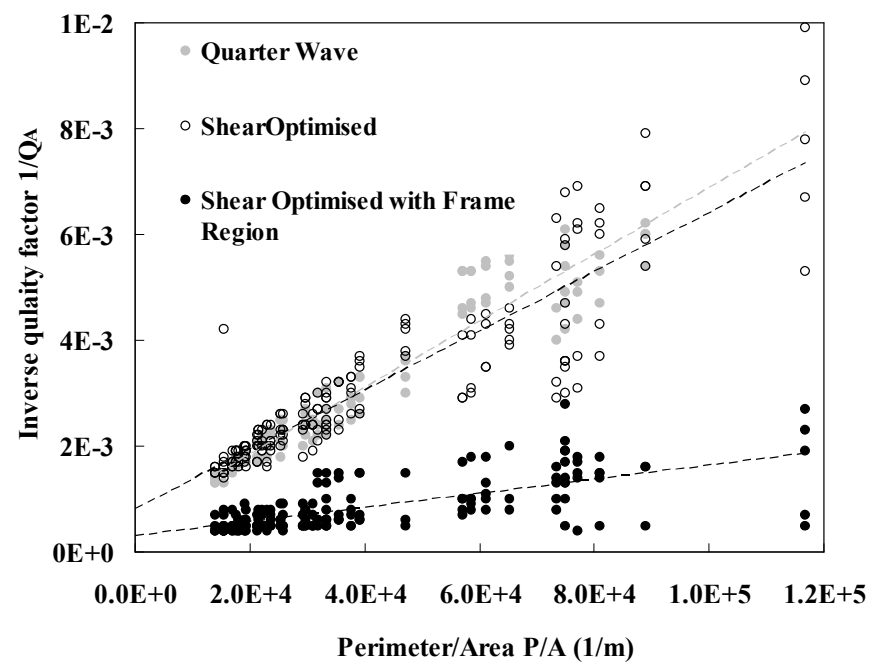

Figure 5.5: $\quad$ Experimental data showing scaling of the quality factor at anti- resonance, $Q_{\mathrm{A}}{ }^{\mathrm{mBVD}}$, for resonators with quarter-wave and shear optimized stack both with and without a frame region [7]. The 1D or extrapolated value at the vertical axis gives us $1 / Q_{\mathrm{A}}$ of the active device (corresponding to $Q_{A}=1600$ for quarter-wave and $Q_{\mathrm{A}}=3300$ for optimized stack); the slope of this (scattered) curve gives information about the parasitic $Q_{\mathrm{A}}$ caused by perimeter/edge effects [11]-[12].

quarter-wavelength reflector stack. These spurs make the proper extraction of a quality factor difficult. To extract the substrate losses we should measure the impedance curve of a very large resonator (unlike the small dimension of 300x100 $\mu \mathrm{m}^{2}$ ) or -more practicalplot $1 / Q_{A}$ vs. perimeter/area [11]-[12]. The $1 D Q_{A}$ is extracted using the perimeter/area $(P / A)$ method discussed in Appendix C. A series of SMRs with area varying from $50 \times 50 \mu^{2}$ up to $300 \times 300 \mu^{2}$ were fabricated to assess the influence of area and perimeter on resonator behavior and hence to extract the $1 \mathrm{D} Q_{\mathrm{A}}$.

Figure 5.5 shows the scaling of quality factor [11]-[12] for resonators at anti-resonance $Q_{\mathrm{A}}$ with a quarter-wave stack and a shear optimized stack with and without a frame region. The $Q_{A}$ values are obtained from these experiments after an mBVD fit. The spread on the $1 / Q_{\mathrm{A}}$ appears much larger for the shear optimized one than for the quarter-wave stack, because spurious modes make good extraction of $Q$ difficult. The shear-optimized reflector alone does not give an improvement in $Q_{\mathrm{A}}$, for neither large nor small resonators. This is because the fraction of stored acoustic energy in the shear waves is small, so an improvement of shear-wave reflection has little impact on the overall $Q_{\mathrm{A}}$. Small resonators have more energy in shear waves but their main loss mechanism is edge acoustic radiation rather than loss into the substrate.

The improvement in $Q$ factor can be seen when a frame region is applied for devices with shear optimized stacks. The combination of shear-optimized stacks with a frame region is a known method to decrease loss due to lateral radiation at the device edge and thus to improve $Q_{A}$ for small devices [7]. However for a frame-region device to work, shear wave reflection is a necessary condition [15] as discussed in section 2.6. The scattering of the graph is strongly reduced by applying the frame region as reported in [10]-[11]. Optimizing the reflector stack for energy confinement thus also allows a straightforward use of the frame region. The extracted 1D quality factor for a resonator with the optimized stack and the frame region is around 3300 and even for small devices

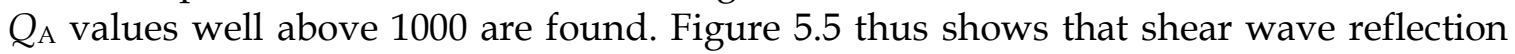
has increased and demonstrates the effectiveness of the proposed optimization scheme. 


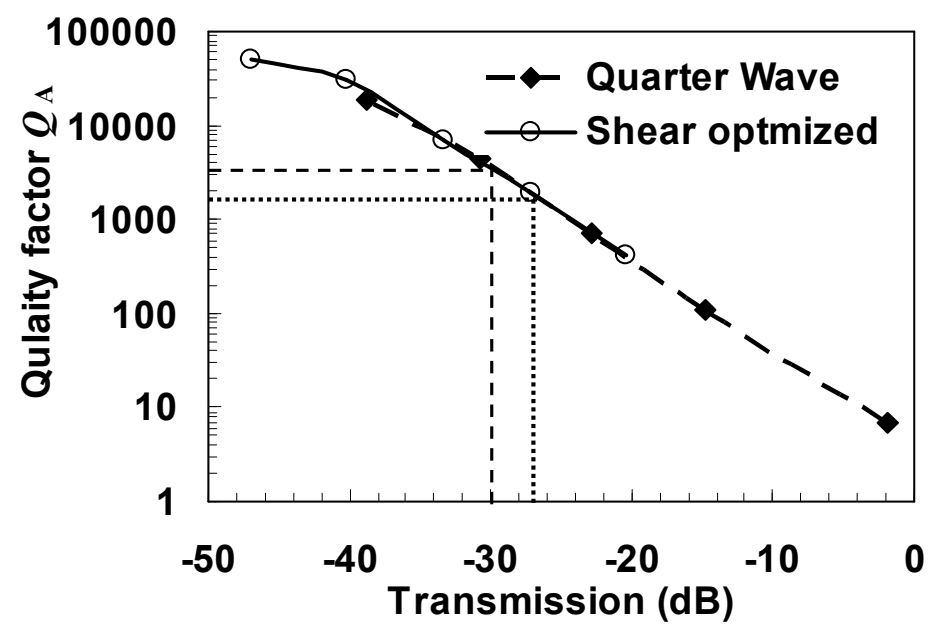

Figure 5.6: $\quad$ Calculated quality factor at anti- resonance $Q_{\mathrm{A}}{ }^{\mathrm{BW}}$, vs. longitudinal transmission for quarter wave and shear optimized $\mathrm{SiO}_{2} / \mathrm{Ta}_{2} \mathrm{O}_{5}$ stacks. The transmission corresponding to $1 \mathrm{D}$ extracted $\mathrm{Q}_{A}$ value (1600) of quarter wave stack is around $-26.5 \mathrm{~dB}$ and that corresponding to shear optimized stack (3300) is around -30dB. This shows the improvement in the $Q_{A}$ value is because of an improved transmission.

In chapter 2 (section 2.4.1), we have seen that transmission of the waves in the stack is related to the $Q$ factor. Hence, a relation between the $1 \mathrm{D}$ extracted $Q_{\mathrm{A}}$ and longitudinal transmission can be derived. This relation depends on the layer configuration of the resonator and can be extracted from the 1D model as follows. First, 1D transmission calculations for a reflector stack with varying number of bi-layers (say 3,5,7,9 etc.) were carried out. Next, 1D device simulations, consisting of the same reflector stack with varying number of bi-layers were carried out to determine $Q_{\mathrm{A}}$. Then a plot of $Q_{\mathrm{A}}$ against transmission is made which can be used to translate the transmission into 1D extracted $Q_{\mathrm{A}}$.

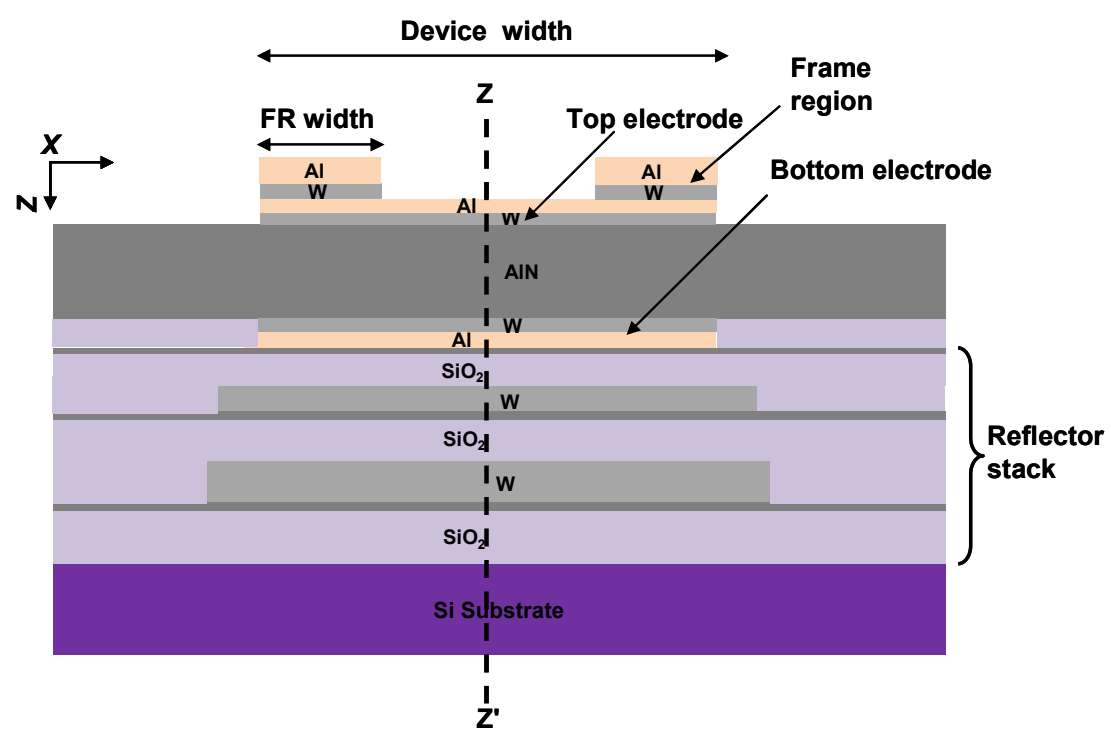

Figure 5.7:

Schematic cross section of an SMR with a five layer $\mathrm{SiO}_{2} / \mathrm{W}$ reflector stack designed using the phase error approach [10].The $W$ layers in the reflector stack are patterned in order to reduce the parasitic capacitances. FR represents the Frame Region. The axis of symmetry assumed in FEM simulations is indicated by the $Z-Z^{\prime}$ line. The drawing is not to scale. 


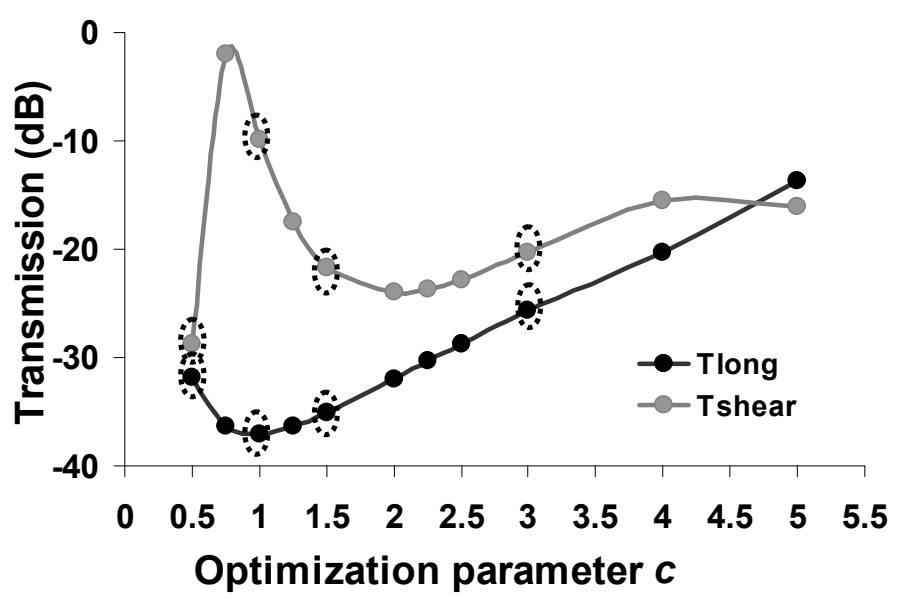

Figure 5.8:

$1 D$ modelled transmission vs. optimization parameter c for a five layer $\mathrm{SiO}_{2} / \mathrm{W}$ reflector stack using material parameters from the external company. The circles indicate the four $c$ values under study.

Figure 5.6 shows an example plot of $1 \mathrm{D} Q_{\mathrm{A}}$ vs. transmission for resonators with a quarter wave stack and a shear optimized stack calculated from the 1D Mason model. Both curves coincide for both quarter wave and shear optimized stacks. This is because $Q_{A}$ vs. transmission is a property of the resonator. Given a resonator and a reflector stack, the quality factor is the ratio of stored energy (that is mostly determined by resonator topology) and the lost energy (loss through the reflector stack conveniently expressed as the transmission). Hence the only way the reflector stack affects $Q_{\mathrm{A}}$ is through the transmission. Therefore it does not matter how a certain $Q_{\mathrm{A}}$ is achieved but it can always be related back to the transmission value.

\section{4 $Q$ improvement of dielectric-metal $\left(\mathrm{SiO}_{2} / \mathrm{W}\right)$ stacks}

In this section, the experimental investigation of two other design approaches, i.e. the stop-band theory based phase error approach (ref. section 3.2) and the diffraction grating method (DGM) (ref. section 3.2), applied to a $\mathrm{SiO}_{2} / \mathrm{W}$ reflector stack is presented. A schematic cross-section of the SMR with stacks designed using the phase error approach is shown in Figure 5.7.

The material choice was because of the high impedance ratio of around 1:7 (with different material parameters from the external company), yielding a high reflection despite of a lower amount of layers $(\mathrm{N}=5)$. However, patterning of the $\mathrm{W}$ layers is required to eliminate the effect of parasitic capacitance [11]. These SMRs have been realized and characterized at an external company. Five different stacks were fabricated: one designed using DGM and the others using four different values of the optimization parameter $c: 0.5,1,1.5$ and 3 . The parameter $c$ is devised for optimizing the thickness of the stacks so as to minimize the longitudinal and shear wave transmissions to the substrate at resonant frequency. The corresponding designed stack thickness, the transmission values from the Mason model (chapter 2) and predicted $Q^{\mathrm{BW}}$ values from the 1D FEM simulations are listed in Table 5.2. Note that viscous losses have an effect on $Q$ factors when the $Q$-regime is above 3000, as reported earlier [5]. 


\begin{tabular}{lccccc}
\hline \hline Stack & $c=0.5$ & $c=1$ & $c=1.5$ & $c=3$ & DGM \\
\hline $\mathrm{SiO}_{2} / \mathrm{W}(\mathrm{nm})$ & $1132 / 500$ & $837 / 738$ & $663 / 880$ & $410 / 1085$ & $2240 / 660$ \\
$T_{\text {long }}(\mathrm{dB})$ & -32 & -37.1 & -35.2 & -26.1 & -37.4 \\
$T_{\text {shear }}(\mathrm{dB})$ & -27.4 & -9.76 & -22.7 & -23.8 & -27.8 \\
$Q^{\mathrm{BW}_{1 \mathrm{D}}}$ & 2522 & 5080 & 4058 & 775 & 4125 \\
$Q^{\mathrm{BW}_{1 \text { Ddamping }}}$ & 2254 & 2540 & 2254 & 650 & 2946 \\
\hline \hline
\end{tabular}

Table 5.2. Stack thicknesses from the phase error approach, the predicted longitudinal and shear transmission values from analytical model and the predicted $Q_{1 D}$ from $F E M$ simulations. $Q_{1 D}$ and $Q_{1 D d a m p i n g}$ are the $Q$ values with and without appropriate viscous losses (see Appendix $A$ ).

Figure 5.8 presents the calculated transmission of longitudinal and shear waves against the optimization parameter $c$ for a five layer $\mathrm{SiO}_{2} / \mathrm{W}$ reflector stack using material parameters from the external company. The best transmission performance is expected when $1<c<2$ [10]. We considered four $c$ values to study the behavior of the stacks as predicted by the theory; $c=1.5$ a value within the best performance regime, and two values outside it i.e. $c=0.5$ and $c=3$. The $c=1$ stack was also chosen because of its almost quarter-wave like characteristics: a good transmission for the longitudinal wave $\left(T_{\text {long }}=-37.1 \mathrm{~dB}\right)$ and a poor transmission for the shear wave $\left(T_{\text {shear }}=-9.76 \mathrm{~dB}\right)$. Hence this stack was intended to serve as a good reference for comparing the shear optimization.

The top-oxide was increased in order to achieve a type I dispersion curve for the direct implementation of the frame region [15]-[16] except for the $c=0.5$ stack. For $c=0.5$, the top-oxide from the design was thick enough $(1132 \mathrm{~nm})$ for the dispersion to be type I. The five layer $\mathrm{SiO}_{2} / \mathrm{W}$ stacks optimized for $1.95 \mathrm{GHz}$ with $\mathrm{AlN}$ as the piezoelectric layer were realized.

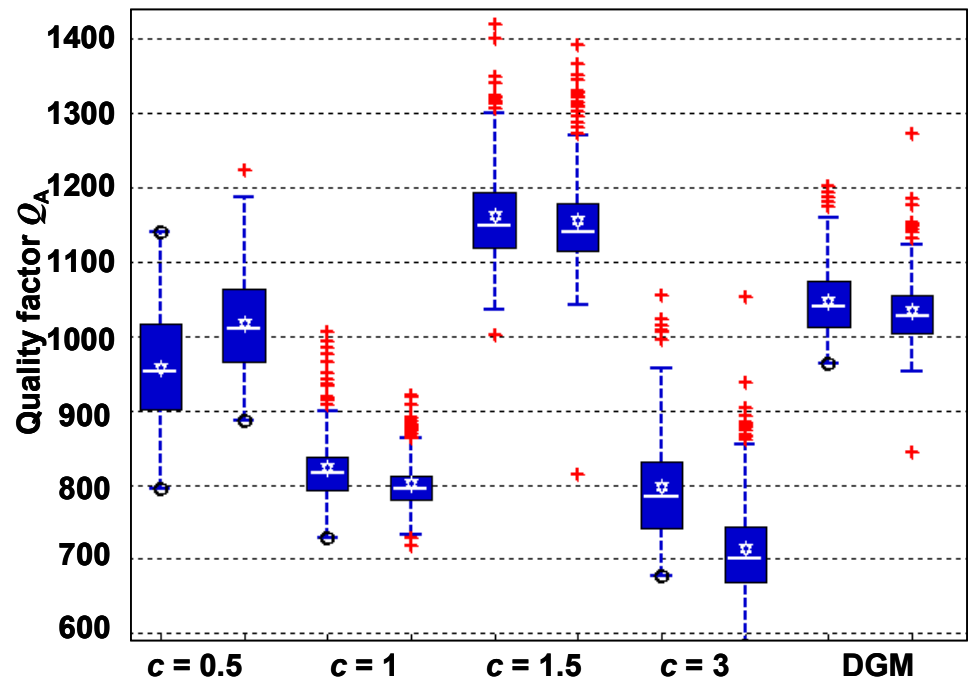

Figure 5.9: $\quad$ Box plot of quality factor at anti-resonance $Q_{A}{ }^{\mathrm{mBV}}$ for fabricated SMRs with various reflector stacks before the frame region deposition.926 devices were measured in each case [courtesy external company]. 


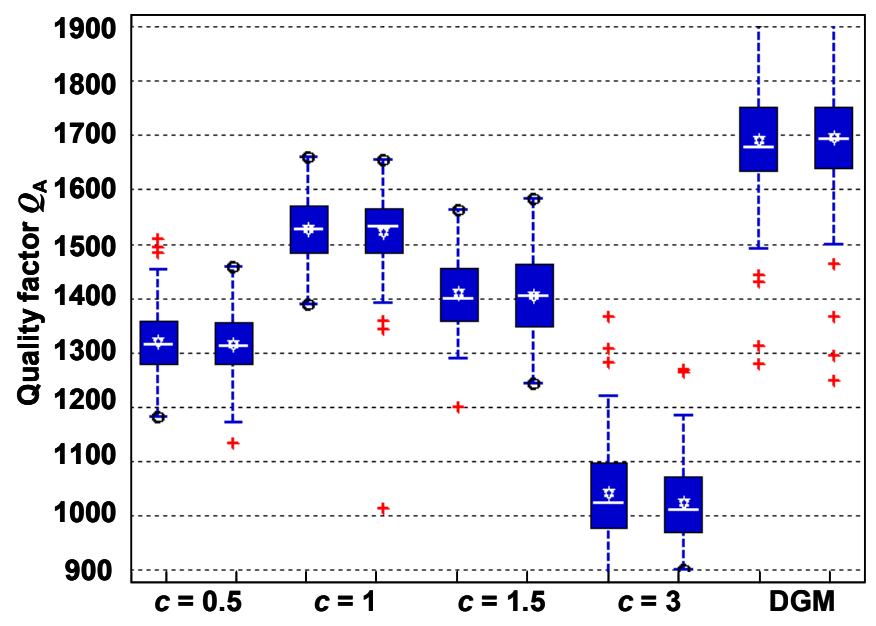

Figure 5.10: $\quad$ Box plot of quality factor at anti-resonance $Q_{\mathrm{A}}{ }^{\mathrm{MVD}}$ for fabricated SMRs with various reflector stacks after the frame region deposition. 926 devices were measured in each case [courtesy external company].

A small frequency step of $1 \mathrm{MHz}$ was used around the resonant frequency to extract the $Q$-factor from the impedance curve using the traditional 3-dB bandwidth method (see eq. 2.17). For the experimental $Q_{\mathrm{A}}$ values an mBVD fit was performed to avoid the effect of frequency sensitivity.

\subsection{1 $Q$ factor Analysis}

Figure 5.9 shows the box plot of $Q_{\mathrm{A}}{ }^{\mathrm{mBVD}}$ extracted with the mBVD model (see section 2.2.2) for the five different fabricated SMRs before the deposition of the frame region. Without the frame region, the measured $Q_{\mathrm{A}}$ values are actually low. The improvement in $Q$ factor can be seen when the frame region is applied for devices with shear optimized stacks because the frame region suppresses spurious modes; the spurious modes are more pronounced when there is more shear confinement in the devices. If a frame region was applied to poor shear reflecting stacks $(c=0.5, c=1$ and $c=3)$, or ideally the quarter-wave stack, there would not be much improvement in $Q_{\mathrm{A}}$ because of the poor shear confinement in the device.

Figure 5.10 shows the box plot of the $Q_{\mathrm{A}}$ for the SMRs after the frame region deposition. A significant improvement of $Q_{\mathrm{A}}$ is seen for $c=1, c=1.5$ and DGM stacks which is again an indication of shear optimization in the stacks. The reason for the unexpected improvement of $Q_{\mathrm{A}}$ in the $c=1$ stack, the quarter-wave like stack, which shows a better $Q_{\mathrm{A}}$ than the optimized stacks will be discussed in section 5.4.1.2. A detailed analysis of DGM stacks which show the highest experimental $Q_{A}$ of 1700 will be presented in section 5.4.2. A small improvement of $Q_{\mathrm{A}}$ is also seen in the case of poor reflecting $c=0.5$ and $c=3$ stacks, which can be attributed to the increased top-oxide layer thickness. The trend in overall $Q_{\mathrm{A}}$ is in line with the theoretical transmission values listed in Table 5.2, except for the $c=1$ stack. 
A series of SMRs with various areas were fabricated to extract the $1 \mathrm{D} Q_{\mathrm{A}}$ using the $P / A$ scaling method described in Appendix C. The area was varying between $100 \mu \mathrm{m}^{2}$ and $550 \mu \mathrm{m}^{2}$ with a frame region width of $3 \mu \mathrm{m}$. Figure 5.11 shows $1 / Q_{\mathrm{A}}$ vs. $P / A$ for the fabricated stacks designed using the phase error approach. Different from Figure 5.5, Figure 5.11 shows the average value of $1 / Q_{\mathrm{A}}$ for the same set of devices in the wafer plotted against $P / A$. Moreover, the scattering of the points are less compared to Figure 5.5 because of the use of a frame region close to the optimum width for spurious suppression which allows a reliable extraction of $Q_{\mathrm{A}}$. The extracted $1 \mathrm{D} Q_{\mathrm{A}}$ also shows the expected trend as in Table 5.2 except for $c=1$ (quarter-wave like stacks; as will be discussed in section 5.4.1.2). Figure 5.12 shows a plot of extracted $1 \mathrm{D} Q_{\mathrm{A}}$ from the measurements and simulations against modelled transmission values. Figure 5.13 shows the plot of modeled $1 \mathrm{D} Q_{\mathrm{A}}{ }^{\mathrm{BW}}$ against transmission values for varying number layers for stacks with different $c$ values. For various $c$ values, a part of the $Q_{\mathrm{A}}$ vs. transmission curves coincides on one line as seen for the $\mathrm{SiO}_{2} / \mathrm{Ta}_{2} \mathrm{O}_{5}$ stack (Figure 5.6). This forms an additional support that the $Q_{\mathrm{A}}$ vs. transmission is a property of the resonator irrespective of the reflector stack. The saturation of $Q_{\mathrm{A}}$ value to just below $10^{5}$ as seen in Figure 5.13 is because of the insufficient frequency points. The modeled $1 \mathrm{D} Q_{\mathrm{A}}{ }^{\mathrm{BW}}$ values are high compared to the simulations because the viscous losses have not been incorporated in the Mason model.

It is interesting to note that the experimental curve in Figure 5.12 nearly follows a linear behavior as seen in the simulations and the model. The $c=0.5$ stack is slightly off-shifted from the straight-line in the experimental curve because in this particular stack the increased top-oxide layer thickness was inherent from the design and hence was not additionally increased.

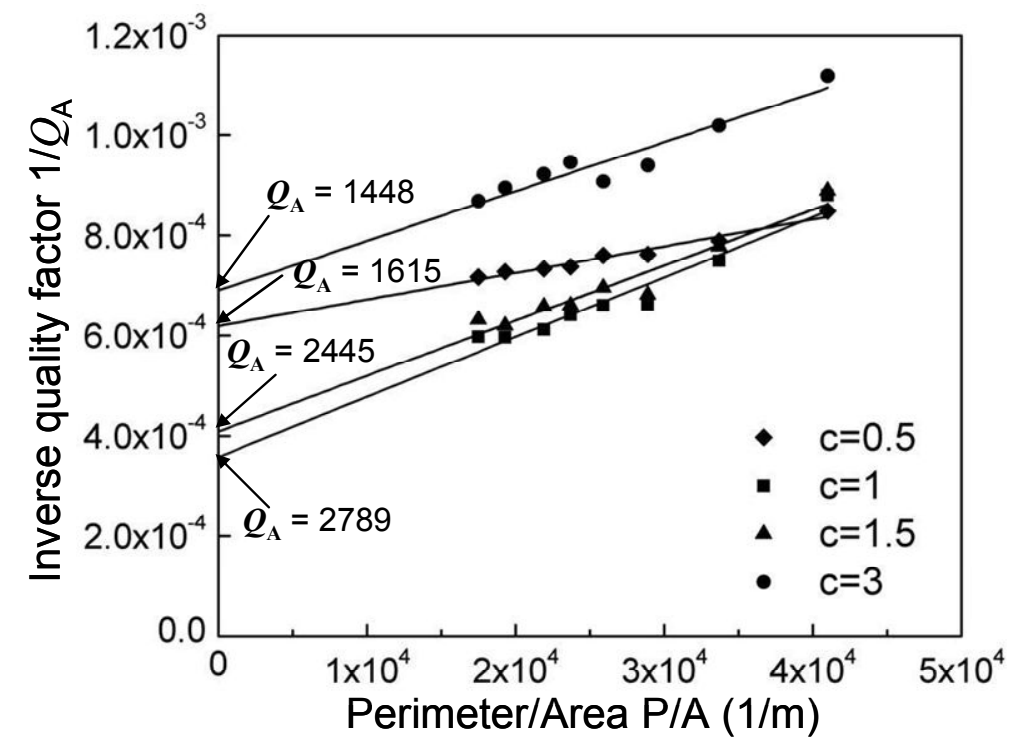

Figure 5.11: $\quad$ Scaling of $Q_{A}{ }^{\mathrm{mBVD}}$ for the fabricated resonators with different reflector stacks designed with the phase error approach for $f_{R}=1.95 \mathrm{GHz}$. The area was ranging from $100 \mathrm{\mu m}^{2}$ to $550 \mathrm{\mu m}^{2}$ with a frame region width of $3 \mu \mathrm{m}$. The extrapolated value at the vertical axis gives us $1 / Q_{\mathrm{A}}$ of the active device. Except for $c=1$ the extracted $1 D Q_{A}$ values are in-line with the simulated transmission values from the scheme shown in the table 5.2. 


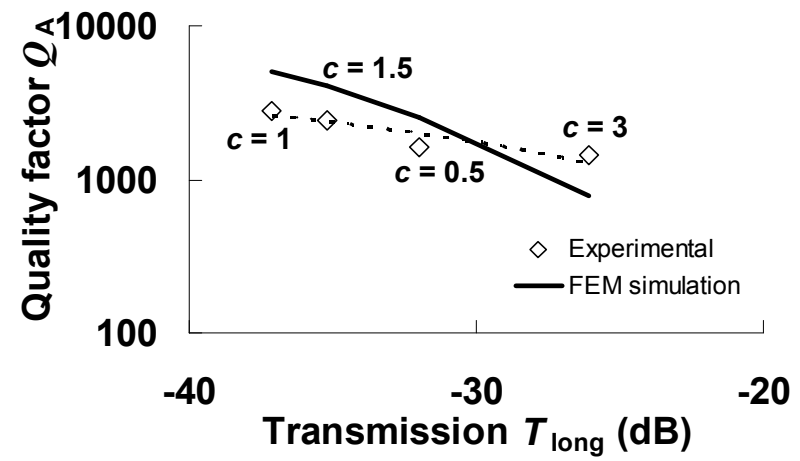

Figure 5.12: $\quad$ Modelled longitudinal transmission against simulated $Q_{A}{ }^{\mathrm{BW}}$ and experimental $Q_{A}{ }^{\mathrm{mBVD}}$. For the experiments, the curve forms a straight line except for $c=0.5$ in which the increased top-oxide was inherent from the design. The dashed line is the fitted line including all the points.

\subsubsection{2D FEM analysis of SMRs with phase error approach based stacks}

A 2D FEM physical model [18] of the SMR with $\mathrm{SiO}_{2} / \mathrm{W}$ stacks for the four different $c$ values was developed to get an insight of the propagation of the waves inside the device. Half structure simulations were done applying symmetry boundary condition $\left(U_{x}=0, \partial U_{z} / \partial x=0\right)$ at the centre of the device indicated by the $Z-Z^{\prime}$ line in Figure 5.7. The only discontinuous layer was the top electrode (with free edge boundaries). At the right hand side, the vertical and horizontal displacements were assumed to be zero $\left(U_{x}=U_{z}=0\right)$. In addition, absorbing boundaries were also used at the right hand side in order to avoid generation of standing waves due to the reflection at the side of the resonator [9], [19] (see also Appendix A).

Figure 5.14 shows the simulated displacement profiles of longitudinal and shear waves at the resonant frequency for the SMRs with stacks designed using the phase error approach with optimization parameter (a) $c=0.5$, (b) $c=1.0,(\mathrm{c}) c=1.5$ and (d) $c=3$. The

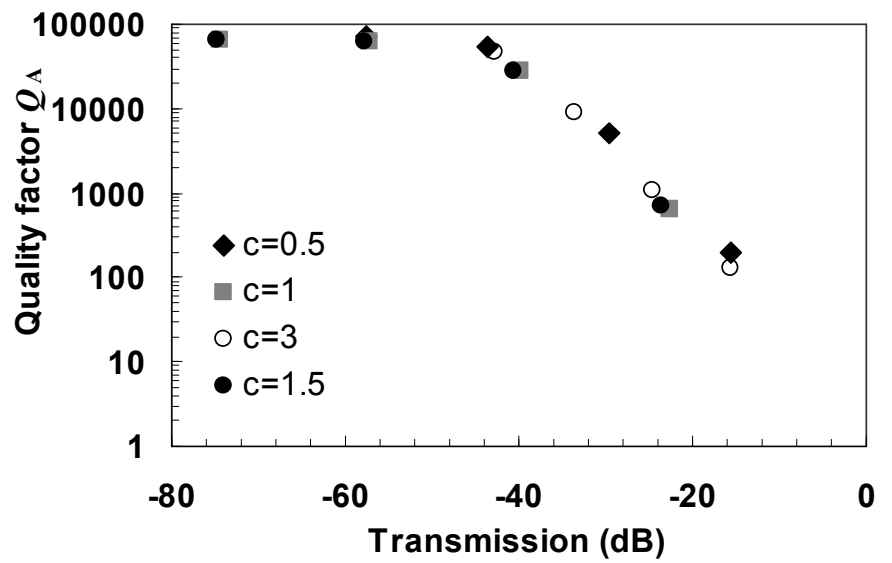

Figure 5.13: Simulated quality factor at anti- resonance $Q_{A}{ }^{B W}$ vs. longitudinal transmission for $\mathrm{SiO}_{2} / \mathrm{W}$ stacks obtained with various values of the optimization parameter $c$. 
displacement profiles illustrate the effect of the stack obtained for various $c$ values. The simulations were performed for a frame region width of $3 \mu \mathrm{m}$, as in the case of fabricated devices, to compute $Q$ from 2D simulations. Note that in these simulations, the top-oxide layer thickness was increased to $1000 \mathrm{~nm}$ according to the rule of thumb explained in section 4.2 for flipping the dispersion curve from type II to type I. 


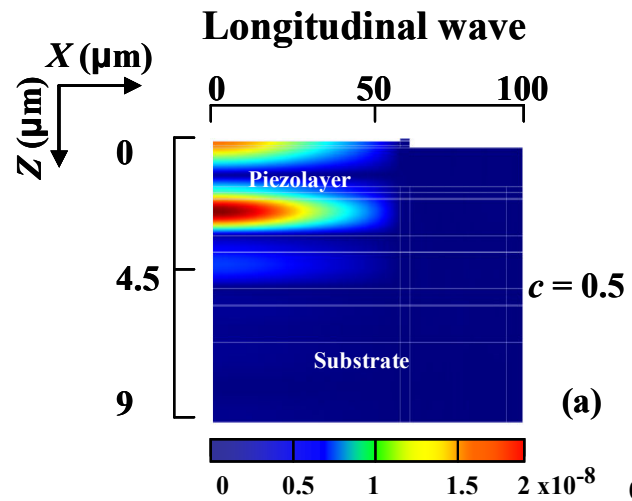

\section{Shear wave}
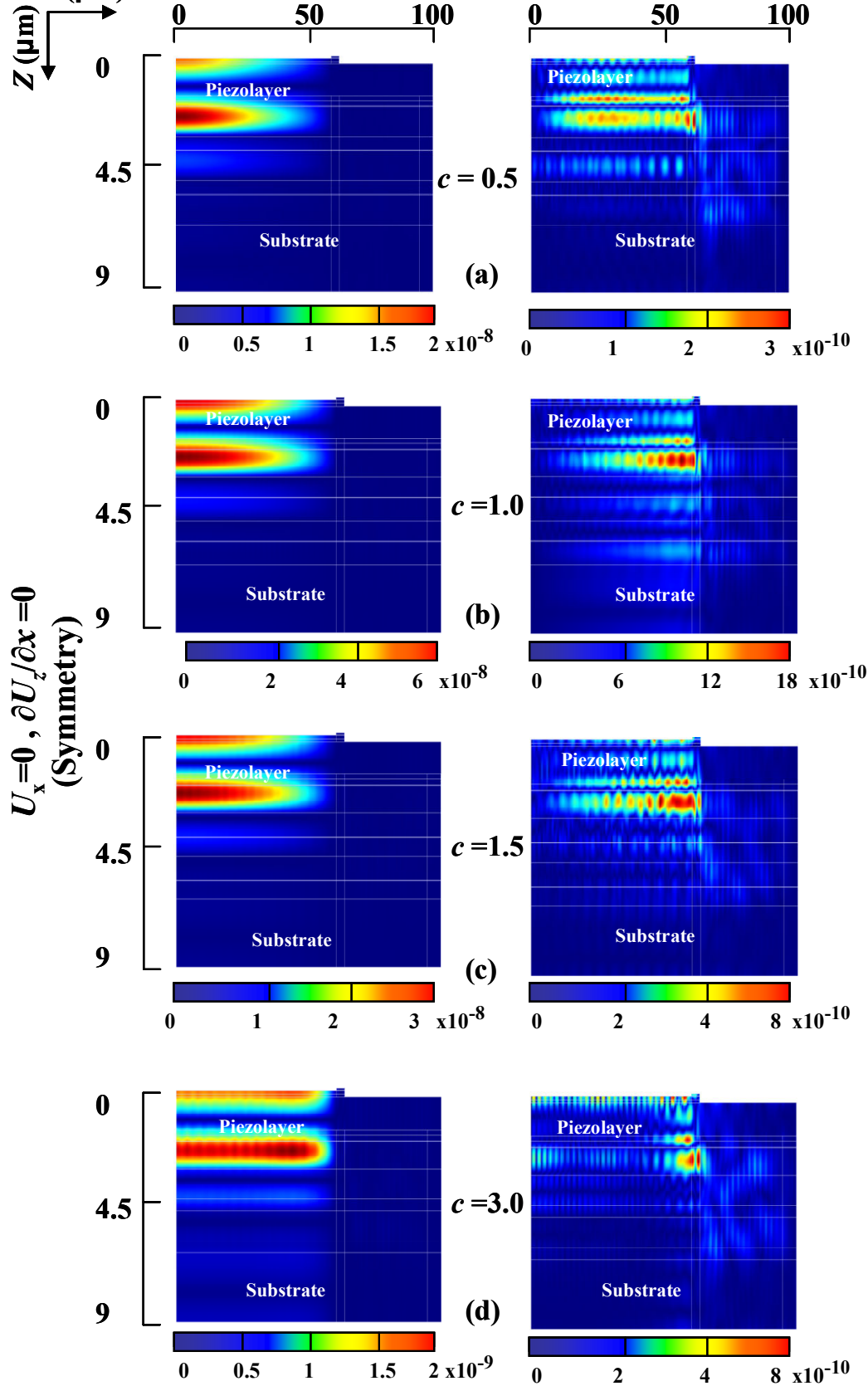

\section{Bottom :Free}

Figure 5.14:

2D FEM results of the vertical displacement of longitudinal waves (left) and shear waves (right) at resonant frequency $f_{R} \approx 2.02 \mathrm{GHz}$ of the $\mathrm{SMR}$ with a 5 layer $\mathrm{SiO}_{2} / W$ reflector stack designed for various $c$ values of the phase error approach (a) $c=0.5$, (b) $c=1.0$, (c) $c=1.5$ and (d) $c=3$. Plane of symmetry is defined at the left edge of the device. Note that although fig (d) features more or less uniformly distributed longitudinal displacement profile, the displacement magnitude is one order of magnitude less and hence $c=3$ is the poorest reflecting stack. Fig (b), $c=1$ stacks show a good reflection of both longitudinal and shear waves despite of the fact that it was designed to be a quarter-wave like stack. Simulations confirm that optimum reflection of both the waves are achieved when $1<c<2$. Boundary conditions at bottom and top are assumed to be free of stress. Absorbing boundaries were used at the right hand side. 


\begin{tabular}{lcccccc}
\hline \hline Stacks & $Q_{\mathrm{A}_{2 \mathrm{DFM}}}^{\mathrm{BW}}$ & $Q_{\mathrm{A}_{2 \mathrm{DFEM}}}^{\mathrm{mBVD}}$ & $Q_{\mathrm{A}_{\text {measured }}}^{\mathrm{mBVD}}$ & $Q_{\mathrm{A}_{1 \mathrm{D} \mathrm{extracted}}}^{\mathrm{mBVD}}$ & $\mathrm{FOM}$ & $\begin{array}{c}\text { Stored } \\
\text { Energy } \\
(\mu \mathrm{J})\end{array}$ \\
\hline$c=0.5$ & 2029 & 2376 & 1310 & 1615 & 72.05 & 282.75 \\
$c=1$ & 2540 & 2337 & 1520 & 2789 & 95 & 412 \\
$c=1.5$ & 2254 & 2162 & 1400 & 2445 & 85.4 & 339 \\
$c=3.0$ & 650 & 707 & 1200 & 1448 & 67.8 & 29.42 \\
\hline \hline
\end{tabular}

Table 5.3:

A comparison between measured $Q_{\mathrm{A}}$ and the $Q_{\mathrm{A}}$ obtained from $2 D$ FEM simulations for the SMRs with different $c$ stacks. The $Q_{\mathrm{A}}{ }^{\mathrm{MVD}}$ is the obtained after an $\mathrm{mBVD}$ fit from the measurements and $2 D$ FEM simulations; $Q_{\mathrm{A}}{ }^{\mathrm{BW}}$ is calculated using the bandwidth method from FEM simulations. The stored energy is the energy stored in the piezoelectric layer obtained from FEM simulations. Appropriate viscous losses (see Appendix A) were incorporated in the $2 D$ FEM simulations. The $1 D$ extracted $Q_{\mathrm{A}}$ values are obtained from Figure 5.11. FOM refers to the figure of merit as mentioned in section 2.3.3.

It is observed in Figure 5.14 that the amplitude of shear displacement in the resonators in the cases of $c=1, c=1.5$ and $c=3$ reflector stacks is less compared to $c=0.5$ stacks. However in the case of $c=3$ stack, the longitudinal displacement amplitude is one order
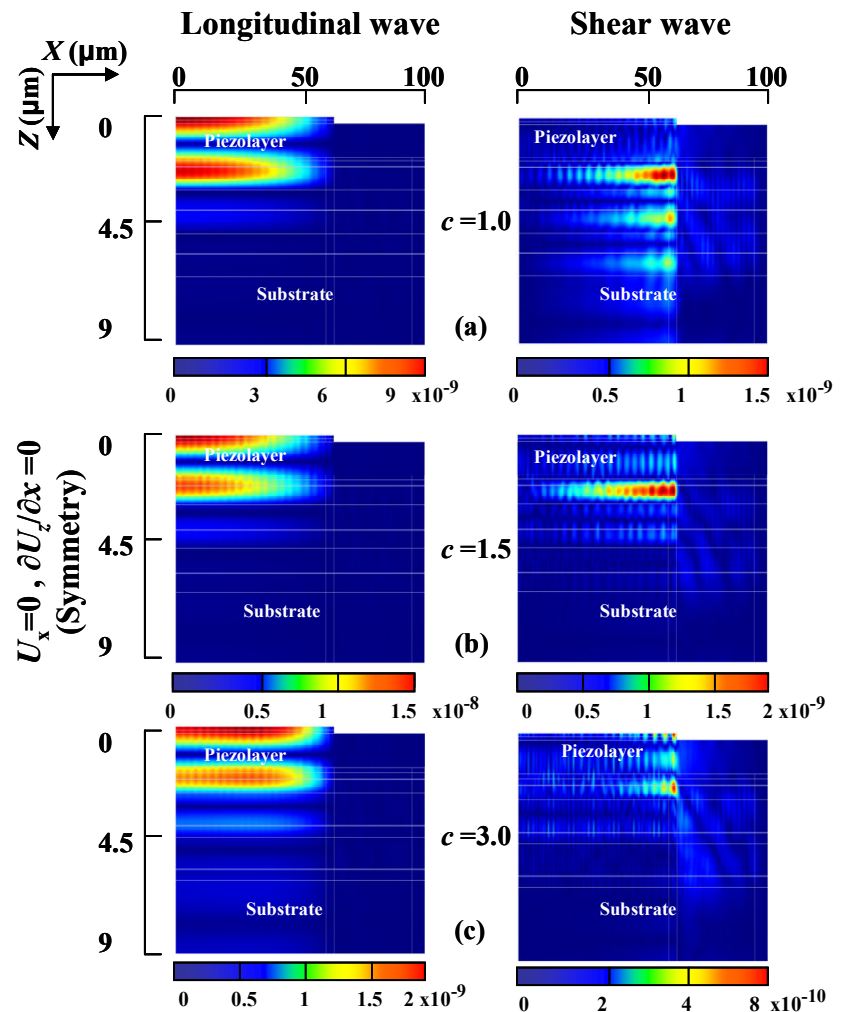

Bottom :Free

Figure 5.15: $\quad 2 D$ FEM results for displacement of longitudinal waves (left) and shear waves (right) at resonant frequency $f_{R} \approx 2.02 \mathrm{GHz}$ of an $\mathrm{SMR}$ with a 5 layer $\mathrm{SiO}_{2} / \mathrm{W}$ reflector stack designed using various $\mathrm{c}$ values of phase error approach (a) $c=1.0$, (b) $c=1.5$ and (c) $c=3$ without the increased top-oxide. Note that in fig (a), the quarter-wave like reflecting stack, there is significant leakage of shear to the substrate. Boundary conditions at bottom and top are assumed to be free of stress. Absorbing boundaries were used at the right hand side. 
of magnitude less which indicates less stored energy. Hence it can be concluded that optimum reflection of both the waves is achieved when $1<c<2$. However, Fig (b), $c=1$ stacks show a good reflection of both longitudinal and shear waves despite of the fact that it was designed to be a quarter-wave like stack. The increase in shear reflection is due to the increased top-oxide layer thickness, which is a deviation from the designed top-oxide thickness, intended for changing the dispersion relation to type I as discussed in section. 5.4.1.2. Note that although Figure 5.14 (d) features a nearly uniformly distributed longitudinal displacement profile, the displacement magnitude being one order of magnitude less makes it the poorest reflecting stack.

Table 5.3 summarizes the $Q$ values from 2D FEM simulations in comparison with the measured $Q$ values with the frame region (ref. Figure 5.11). Simulations and the measurements show the same trend although the 2D $Q$ values are quite high. $Q$ values are obtained from the impedance curves using the bandwidth method. However, the $Q$ values from the measurements are obtained after doing an $\mathrm{mBVD}$ fit and hence we compared $Q_{\mathrm{A}}$ values estimated from FEM simulations after doing an $\mathrm{mBVD}$ fit as well. The $Q_{\mathrm{A}}$ value obtained for $c=0.5$ after the mBVD fit is slightly higher than that of $c=1$ and $c=1.5$ cases. This is because of the spurious modes at anti-resonance which made the extraction difficult in the case of $c=0.5$. In order to substantiate the FEM simulation results, we also verified the stored energy in the piezoelectric layer obtained from FEM simulations. This entirely follows the theoretical trend. Since the same method of calculation is implemented for the various stacks, the trend in the calculated $Q$ factor still holds.

The inaccuracy of the material parameters used in the 2D model for these particular devices could also be a reason for the difference in the resonance frequency obtained from the measurements $\left(f_{\mathrm{R}} \approx 1.95 \mathrm{GHz}\right)$ and the simulations $\left(f_{\mathrm{R}} \approx 2.02 \mathrm{GHz}\right)$. Another reason could be that the measured $Q_{A}{ }^{m B V D}$ values are from SMRs having finite dimensions (and hence 3D) and therefore there are more losses involved. It can be seen from Table 5.3 that except for $c=1$, the 1D extracted $Q_{\mathrm{A}}$ values obtained from measurements as well as 2D FEM simulations are in-line with the trend in the calculated transmission values from the scheme, also shown in Table 5.2. Optimum performance was expected for $c=1.5$ from the 1D model, however for $c=1$, quarter-wave like stacks with an increased top-oxide layer thickness, showed a higher $Q_{\mathrm{A}}$ in $2 \mathrm{D}$ simulations and in experiments. This will be explained in the next section.

\subsubsection{Influence of the top-oxide layer on phase error approach based stacks}

The improvement of $Q_{\mathrm{A}}$ in the $c=1$ stack motivated us to investigate the effect of the top-oxide layer in the stacks designed by phase error approach. The 2D FEM simulations were performed for various c stacks $((\mathrm{a}) c=1.0,(\mathrm{~b}) c=1.5$ and $(\mathrm{c}) c=3$ ) without any topoxide increase and the results are shown in Figure 5.15. The top-oxide layer thickness was not increased in the $c=0.5$ stack because the top-oxide layer from the phase error approach was thick enough $(1132 \mathrm{~nm})$ for the dispersion curve to be type I. Note that in these simulations there is no frame region as the frame region has an effect only when the dispersion is type I [15]. 
In Figure 5.15 (a) in which the quarter-wave like reflector stack is employed, there is a significant leakage of shear waves to the substrate compared to Figure 5.14(b) in which the top-oxide layer thickness is increased for flipping the dispersion curve. With the increased top-oxide layer in the quarter-wave like reflecting stack, the shear transmission has been improved. In the case of the $c=1.5$ stack, the top-oxide has not influenced the longitudinal transmission much. However, the intensity of the shear wave has increased to some extent but not adverse enough to affect the $Q_{\mathrm{A}}$, shear not being leaking into the substrate. In the case of the $c=3$ stack, which was the poorest of the reflecting stacks, the top-oxide does not have any influence. Hence it can be concluded that the increase of top-oxide layer thickness has an effect on the shear wave leakage in the stacks only if the stack is a reasonably good longitudinal reflector with a minimum longitudinal transmission, $\mathrm{T}_{\text {long }}=-30 \mathrm{~dB}$ (see Table 5.2). Consequently, for a frame-region device to work, shear wave reflection is a necessary condition [15].

2D FEM simulations were also performed on longitudinal quarter-wave stacks at $1.99 \mathrm{GHz}$ with and without an increased thickness of the top-oxide layer. The simulation results are shown in Figure 5.16. The simulations reveal that the quarter-wave stack with an increased top-oxide layer thickness has an improved shear reflection. The increased top-oxide acts as a cavity for shear resonance and shear transmission is significantly improved. For a better comparison, the 1D displacement profiles of longitudinal and shear waves from FEM simulations for a conventional quarter wave mirror and the one with an increased top-oxide layer are shown in Figure 5.17. It can be seen from the figure that for the quarter-wave stack with the increased oxide layer thickness, shear wave displacement in the device is minimum, without the longitudinal wave displacement being much affected. In this case, the shear displacement in the piezolayer is much larger preventing the further leakage of shear to the substrate, compared to the conventional quarter-wave stack.

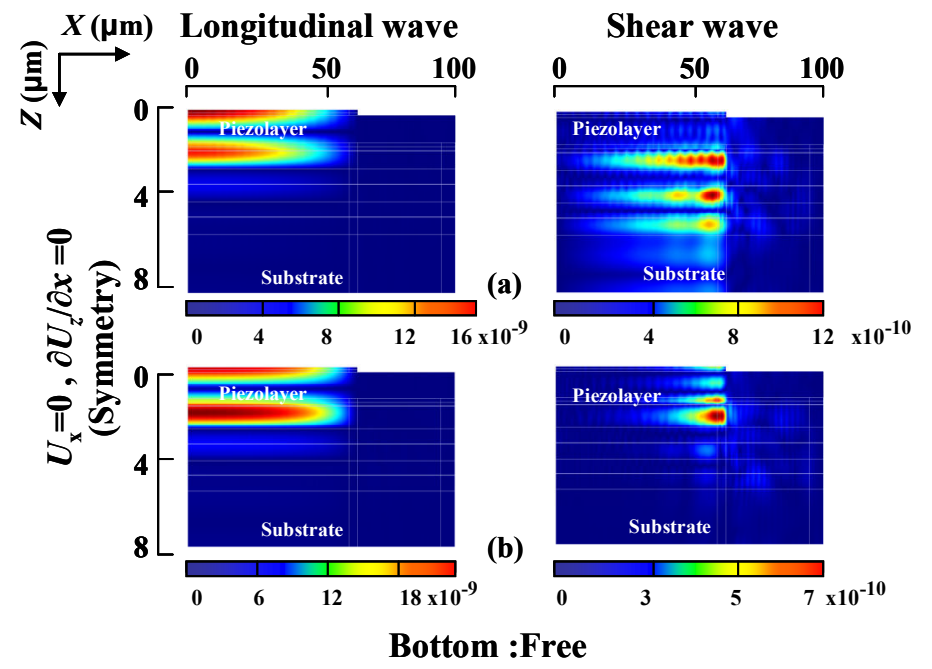

Figure 5.16: $\quad 2 D$ FEM results for the vertical displacement of longitudinal waves (left) and shear waves (right) at resonant frequency of an $\mathrm{SMR}$ with a five layer $\mathrm{SiO}_{2} / W$ reflector stack using a quarter-wave stack at $1.99 \mathrm{GHz}$ (a) without and (b) with an increased top-oxide. Plane of symmetry is defined at the left edge of the device. Boundary conditions at bottom and top are assumed to be free of stress. Absorbing boundaries were used at the right hand side. 


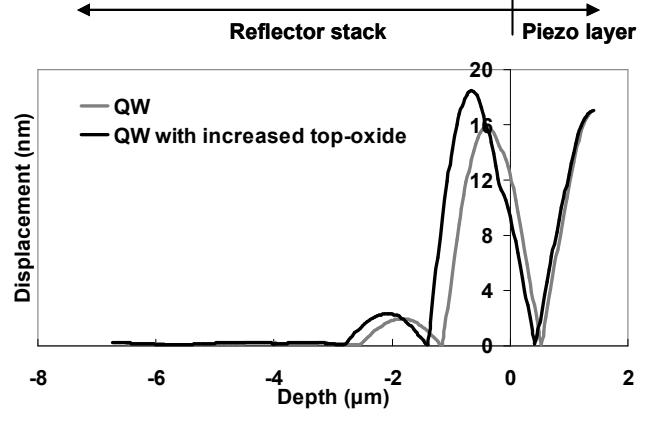

(a)

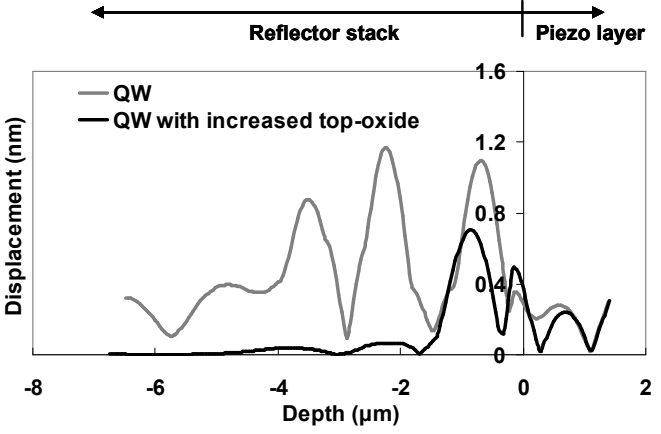

(b)

Figure 5.17:

The vertical displacement profile of (a) longitudinal waves and (b) shear waves in a conventional quarter-wave stack (gray) and the quarter-wave stack with an increased top-oxide (black) at resonant frequency of an $\mathrm{SMR}$ with a five layer $\mathrm{SiO}_{2} / \mathrm{W}$ reflector stack optimized at $1.99 \mathrm{GHz}$ obtained from 2D FEM simulations. The horizontal scale is relative to the bottom of the piezoelectric layer.

This effect of increased top-oxide layer thickness on longitudinal and shear transmissions was not predicted by the 1D transmission line model as shown in Figure 5.18. In fact, the work presented in chapter 3 [10] is based on 1D calculations. It is plausible that this effect can be caused by the coupling (via the Poisson ratio) between the shear and longitudinal waves in the oxide incorporated in the 2D FEM simulator. The combination of the quarter-wave stack with a thick top-oxide for shear optimization has not been reported yet. An additional advantage of such a configuration is that the increased top-oxide in the quarter-wave stack makes the dispersion relation to be type I.

A comparison of the impedance curves was also done to study the improvement in device performance. Figure 5.19 shows the absolute value of impedance of the resonator with a conventional quarter-wave reflector and the one with an increased top-oxide layer, both obtained from 2D FEM simulations. The improvement in $Q$ can also be seen by a direct look at the impedance curve of the resonator [13]. In this case, the quarter-

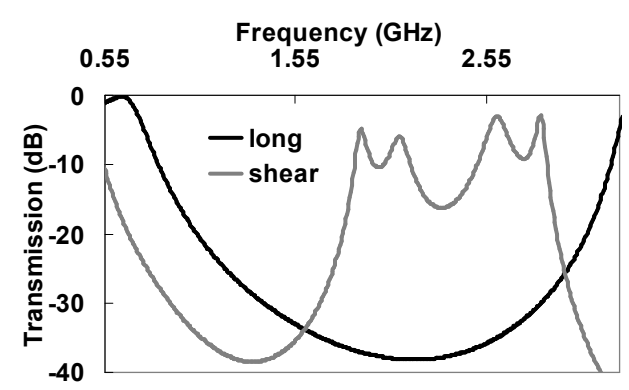

(a)

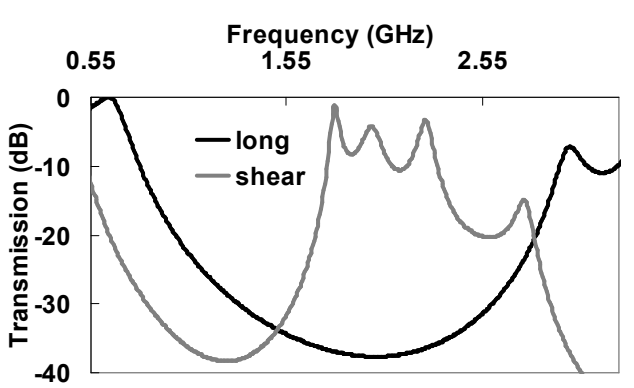

(b)

Figure 5.18:

$1 D$ transmission line modelled transmission curves for longitudinal and shear waves for a conventional quarter-wave stack (left) and the quarter-wave stack with an increased top-oxide layer (right) optimized for a $1.99 \mathrm{GHz}$ SMR with a five layer $\mathrm{SiO}_{2} / \mathrm{W}$ reflector stack. No significant differences are observed in the region of interest from the transmission curves at $f_{R}=1.99 G \mathrm{~Hz}$. 


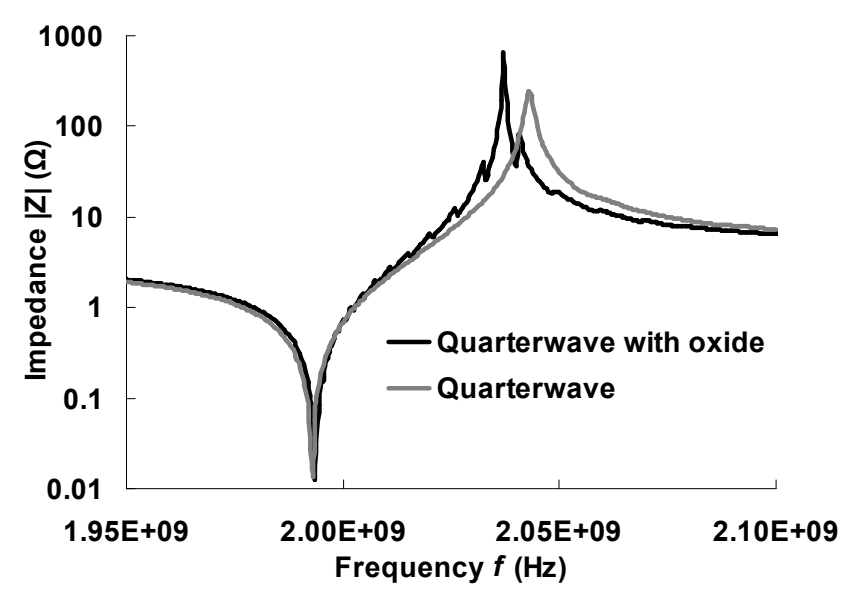

Figure 5.19: Comparison of the impedance curves from 2D FEM simulations for an SMR with a conventional quarter-wave stack (gray) and quarter-wave stack with increased topoxide layer thickness (black) of an SMR with a five layer $\mathrm{SiO}_{2} / \mathrm{W}$ reflector stack optimized at $1.99 \mathrm{GHz}$. Active device area is $140 \times 140{\mu \mathrm{m}^{2}}^{2}$.

wave stack with an increased top-oxide layer thickness enhanced the maximum impedance at anti-resonance by more than a factor of 2 . The impedance curve also shows stronger spurs indicating that the acoustic energy is better confined in the stack with increased top-oxide than in the conventional quarter-wavelength reflector. The calculated $Q_{\mathrm{A}}{ }^{\mathrm{BW}}$ using the bandwidth method (1850) shows indeed an improvement of a factor of two compared to the conventional quarter-wave stack (817). Thus it can be concluded that the $Q$ factor of an SMR employing the conventional quarter-wave stack is significantly improved by increasing the top-oxide layer. However, a reduction in coupling coefficient was also observed.

Although the longitudinal displacement profile in Figure 5.16 (b) is wider than (a), a loss in the coupling coefficient is predicted in the impedance curves. This is attributed to the thicker top-oxide which increased the acoustic energy in this layer and it is not being coupled to the electric field, yielding a reduced coupling coefficient.

The method of improving the shear transmission by an increased top-oxide layer was applied also for $\mathrm{SiO}_{2} / \mathrm{Ta}_{2} \mathrm{O}_{5}$ stacks with a low impedance contrast. However, thickening the top-oxide layer did not lead to an improvement in shear transmission in this case. Hence this method applies only to the case of the material combinations with a high impedance ratio. To put in a broader perspective, the quarter-wave stack with increased top-oxide can be considered as a special case of ADGM stacks. The ADGM method works only in the case of high impedance contrast stacks. The quarter-wave stacks with increased top-oxide layer thickness are similar to ADGM stacks with the exception that the lower layers are quarter-wave thick. In the case of ADGM stacks, the alternate lower layers are multiple quarter-wave thick by design.

\subsubsection{Diffraction grating method stack analysis}

A series of SMRs with a DGM reflector stack were realized for various areas and measured to extract the $1 \mathrm{D} Q_{A^{\mathrm{mBVD}}}$ using the $P / A$ scaling method as done for the phase error approach based stacks. The area was ranging from $100 \mu \mathrm{m}^{2}$ to $550 \mu^{2}$ with a frame 


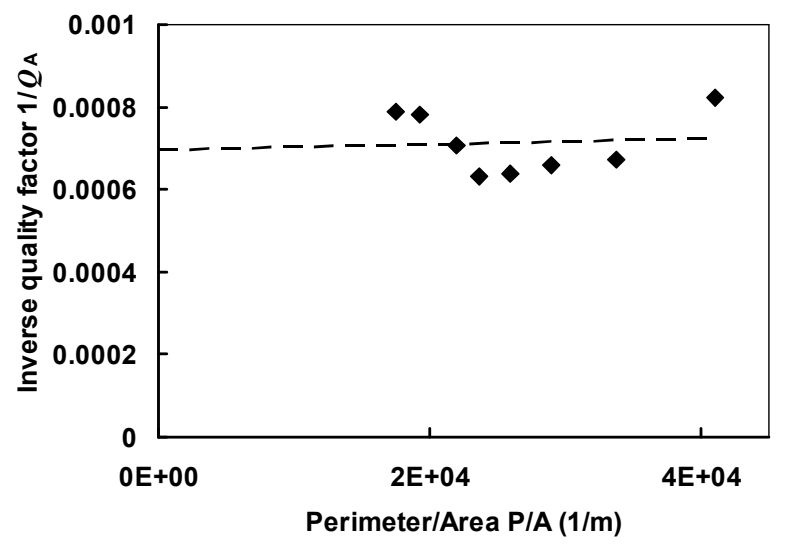

Figure 5.20 : $\quad 1 / Q_{A}{ }^{\mathrm{MBVD}} V S$ P/A for fabricated resonators with $D G M$ designed reflector stacks at $f_{R}=$ $1.95 \mathrm{GHz}$. The area was ranging from $100 \mathrm{\mu m}^{2}$ to $550 \mathrm{\mu m}^{2}$ with a frame region width of $3 \mu \mathrm{m}$. As can be seen, the DGM stack does not show a P/A scaling.

region width of $3 \mu \mathrm{m}$. Figure 5.20 shows $1 / Q_{\mathrm{A}}{ }^{\mathrm{mBVD}}$ vs. $P / A$ for the fabricated SMRs with DGM designed stacks.

In contrast to Figure 5.11, the resonators with the DGM stack do not show any P/A scaling. This means that whatever the device size, the same $Q_{\mathrm{A}}{ }^{\mathrm{mBVD}}(1428)$ is obtained; $Q_{\mathrm{A}}{ }^{\mathrm{mBVD}}$ does not scale with the area. A possible reason for this scenario is that for a frame region width of $3 \mu \mathrm{m}$ under study here, the device has almost a uniformly distributed longitudinal displacement profile (ref. Figure 5.23) [20] in which maximum energy couples to the electric field. The resonators were fabricated for various frame region widths ranging from $1.5 \mu \mathrm{m}$ to $4.25 \mu \mathrm{m}$ in steps of $0.25 \mu \mathrm{m}$ to verify this as shown in Figure 5.21.

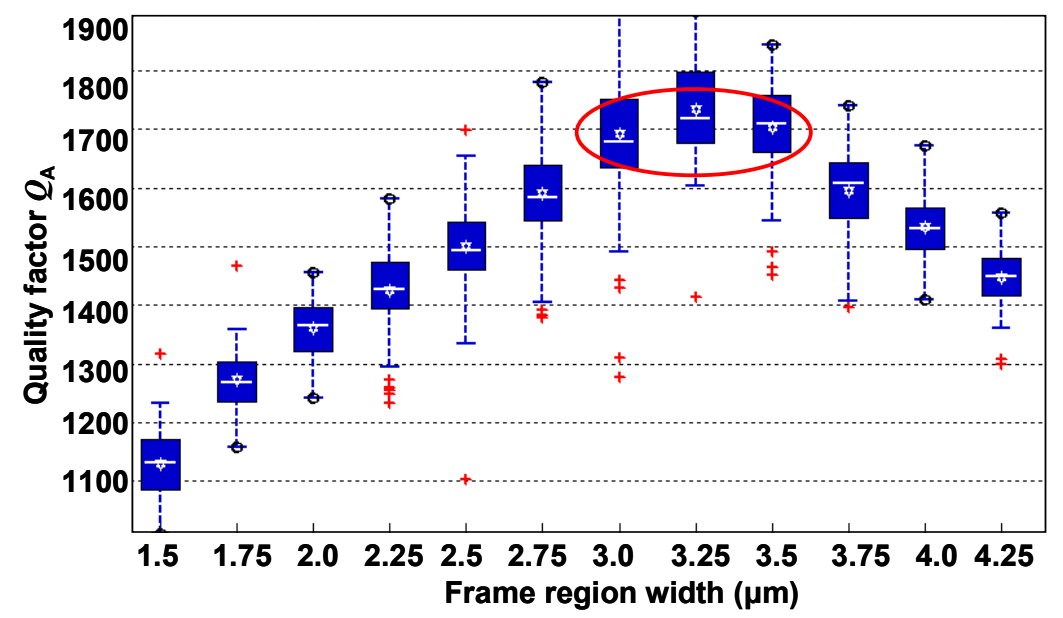

Figure 5.21: $\quad$ Box plot of the experimental quality factor at anti-resonance $Q_{A}{ }^{\mathrm{mBVD}}$ for fabricated SMRs with DGM stacks for frame region width ranging from $1.5 \mu \mathrm{m}$ to $4.25 \mu \mathrm{m}$ in steps of $0.25 \mu \mathrm{m}$. High $Q$ values are obtained for a width of around $3 \mu \mathrm{m}$ [courtesy external company]. 


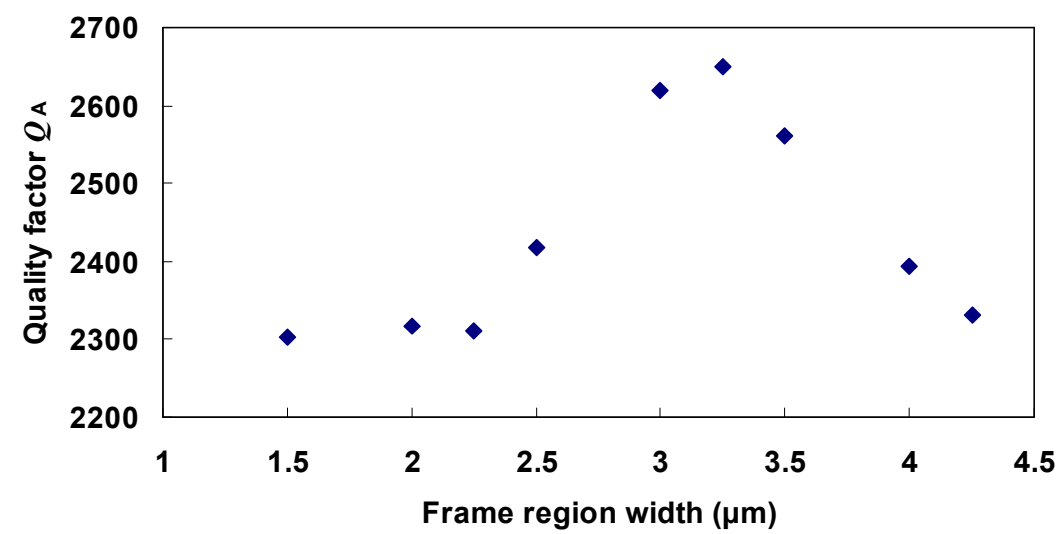

Figure 5.22 : $\quad 2 D$ FEM simulations values of the quality factor at anti-resonance $Q_{A}{ }^{\mathrm{BVD}}$ for $S M R s$ with DGM stacks against the frame region width (compare to Figure 5.21). High $Q$ values are obtained for a width of around $3 \mu \mathrm{m}$.

High $Q$ values are obtained for a frame region width of around $3 \mu \mathrm{m}$. The resonators with the DGM stack showed the highest experimental $Q_{\mathrm{A}}{ }^{\mathrm{mBVD}}$ of 1700 . FEM simulations were also performed for comparison. Figure 5.22 shows the $Q_{\mathrm{A}}{ }^{\mathrm{mBVD}}$ for frame region variations on the 2D FEM simulated SMRs with DGM stacks. Although the $Q_{\mathrm{A}}$ values obtained from 2D FEM simulations are higher (because the real devices are 3D) compared to measured $Q_{\mathrm{A}}$ values, the trend in simulations more or less follows the trend in the measurements. The simulations also show high $Q$ values around frame region width of around $3 \mu \mathrm{m}$.

This instigated us to look at the displacement profile of the structure with the frame region width which resulted in high $Q$, to get a better insight of the acoustic wave propagation inside the device. Figure 5.23 shows the longitudinal and shear displacement profile of the resonator for a frame region width of $3 \mu \mathrm{m}$. It can be seen from Figure 5.23 that the device has a wide longitudinal displacement profile indicating better confinement of energy and thus high $Q$. This could also be a reason why DGM stacks do not show $P / A$ scaling.

\subsection{Conclusions}

Experimental verification of the design approaches for optimizing dual reflection of longitudinal and shear waves in solidly mounted BAW resonators has been presented. All the design approaches; the stop-band theory based approach, the phase error approach and the approach based on the diffraction grating method, discussed in chapter 3 have been validated experimentally. Experiments were done for two material combinations in the reflector stack: $\mathrm{SiO}_{2} / \mathrm{Ta}_{2} \mathrm{O}_{5}$ and $\mathrm{SiO}_{2} / \mathrm{W}$. The results show that the design approaches work for the material combinations considered and hence the versatility of the schemes is established. 

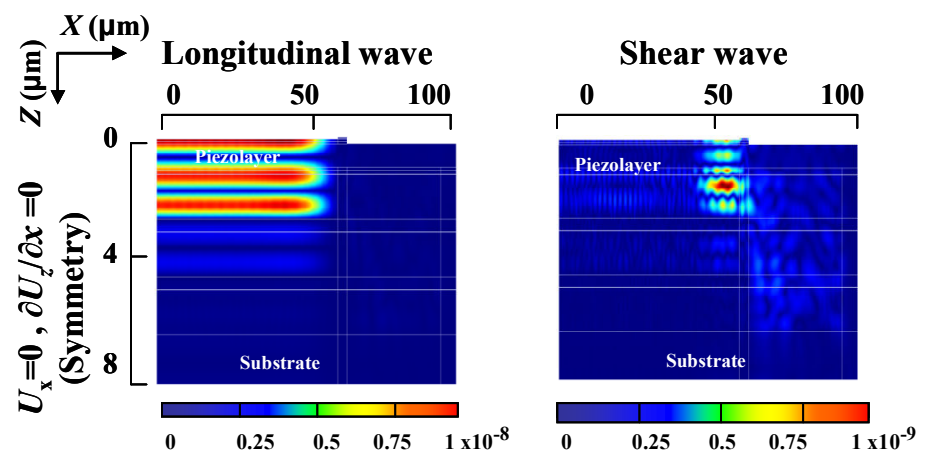

Bottom :Free

Figure 5.23 :

2D FEM results of vertical displacement of longitudinal waves (left) and shear waves (right) in an SMR with a five layer $\mathrm{SiO}_{2} / \mathrm{W}$ reflector stack having a DGM stack at $1.95 \mathrm{GHz}$ for a $3 \mu \mathrm{m}$ frame region width. Plane of symmetry is defined at the left edge of the device. The longitudinal wave displacement shows a uniformly distributed displacement profile. Boundary conditions at bottom and top are assumed to be free of stress. Absorbing boundaries were used at the right hand side.

The resonators based on $\mathrm{SiO}_{2} / \mathrm{Ta}_{2} \mathrm{O}_{5}$ stacks were optimized at $1.88 \mathrm{GHz}$ (US-PCS band: uplink). The experiments demonstrate improved 1D $Q_{A}$ values as high as 3300 . The appearance of spurs in the impedance curves confirms the better confinement of acoustic energy in the resonator. Even for small resonators $Q_{A}$ well above 1000 was found.

The experiments on the resonators with $\mathrm{SiO}_{2} / \mathrm{W}$ stacks were aimed at validating the phase error approach and the diffraction grating approach. These were optimized at 1.95-1.97 GHz (US-PCS band: downlink). The trend of $Q_{\mathrm{A}}$ as a function of the $c$ parameter as predicted by theory is in-line with the experimental $Q_{\mathrm{A}}$ trend except for the $c=1$ stack. Optimum performance was expected for $c=1.5$ from the 1D model, however for $c=1$, the quarter-wave like stack with an increased top-oxide layer thickness, showed a higher extracted 1D $Q_{\mathrm{A}}$. This is due to the possible coupling between the shear and longitudinal waves in the oxide, accounted for in the FEM simulator. 2D simulations confirm that an increased top-oxide layer thickness in the quarter-wave stack considerably decreases shear transmission to the substrate and hence improves the $Q_{\text {A }}$ significantly. This applies only for the high contrast materials, as in the case of ADGM, and hence the quarter-wave stack with an increased top-oxide layer can be considered as an exclusive case of the ADGM approach. The theoretical prediction of the optimum reflection when $1<c<2$ holds.

The resonators with DGM stacks did not show any $P / A$ scaling effect. Therefore, the influence of the frame region width has been studied. High $Q$ values are obtained for a frame region width of around $3 \mu \mathrm{m}$ exhibiting a wide longitudinal displacement profile in the resonator. This could be a reason for $Q_{\mathrm{A}}$ not scaling with the area. It is noticed in the case of $\mathrm{SiO}_{2} / \mathrm{Ta}_{2} \mathrm{O}_{5}$ stacks (Figure 5.5) also, the experiments were done with optimized frame regions which reduced the area dependency of $Q_{\mathrm{A}}$. The resonators 
using $\mathrm{SiO}_{2} / \mathrm{W}$ DGM stacks showed the highest experimental $Q_{\mathrm{A}}$ of around 1700 among the fabricated devices.

\section{References}

[1] S. Jose and R. J. E. Hueting, "Experimental investigation of dual wave optimized reflector stacks in solidly mounted bulk acoustic wave resonators," Proc. IEEE Ultrasonics Symposium, October, 2011.

[2] David. M. Pozar, Microwave Engineering, 3rd ed. John Wiley \&sons, Inc., Hoboken, New Jersey, 2007.

[3] H. Campenella, Acoustic Wave and Electromechanical Resonators: Concepts to key applications, Artech House, 2010.

[4] T. Pensala, Thin Film Bulk Acoustic Wave Devices. Performance Optimization and Modeling, Ph. D Dissertation, VTT Helsinki, Finland, 2011.

[5] K. Hashimoto, RF Bulk Acoustic Filters for communications, Artech House, 2009.

[6] Rohde \& Schwartz International Network Analyzers, http://www2.rohde-schwarz.com.

[7] R. Aigner, "Bringing BAW technology into volume production: The Ten Commandments and the seven deadly sins", IEEE Int. Symp. Acoust. Wave. Dev. for Future Mobile Communication Syst, 2007.

[8] J. Kaitila, "Review of wave propagation in BAW thin film devices progress and prospects", Proc. IEEE Ultrasonics Symposium, pp. 120-129, 2007.

[9] T. Pensala, R. Thalhammer, J. Dekker, and J. Kaitila, "Experimental Investigation of Acoustic Substrate Losses in 1850-MHz Thin Film BAW Resonators", IEEE Trans. on Ultrasonics, Ferroelectrics and Frequency Control, vol. 56, no.11,pp. 2544-2552, 2009.

[10]S. Jose, A. B. M. Jansman, R. J. E. Hueting and J. Schmitz, “Optimized reflector stacks for solidly mounted bulk acoustic wave resonators", IEEE Trans. on Ultrasonics Ferroelectric and Frequency Control, vol.57, no.12, pp. 2753-2763, 2010.

[11]J. W. Lobeek, R. Strijbos, A. B. M. Jansman, N. X. Li, A. B .Smolders and N. Pulsford, "High-Q BAW resonator on $\mathrm{Pt} / \mathrm{Ta}_{2} \mathrm{O}_{5} / \mathrm{SiO}_{2}$-based reflector stack", Proc. IEEE Microwave Symposium, pp. 2047-2050, 2007.

[12] R. Strijbos, A. B. M. Jansman, J. W. Lobeek, N. X. Li and N. Pulsford, "Design and characterization of high-Q Solidly-Mounted Bulk Acoustic Wave filters", Proc. IEEE Electronic components and technology conference, pp. 169-174, 2007.

[13] S. Marksteiner, J. Kaitila, G. G. Fattinger and R. Aigner, “Optimization of acoustic mirrors for Solidly Mounted BAW resonators", Proc. IEEE Ultrasonics Symposium, pp. 329-332, 2005.

[14]F. Z. Bi and B. P. Barber, "Bulk acoustic wave RF technology", IEEE Microwave magazine, pp. 65-80, October 2008.

[15]G. G. Fattinger, S. Marksteiner, J. Kaitila, and R. Aigner, "Optimization of acoustic dispersion for high performance thin film BAW resonators", Proc. IEEE Ultrasonics Symposium, pp. 1175-1178, 2005.

[16] R. Thalhammer, J. Kaitila, S. Zieglmeier and L. Elbrecht, "Spurious mode suppression in BAW resonators", Proc. IEEE Ultrasonics Symposium, pp. 456-459, 2006. 
[17] R. Ruby, "Review and comparison of Bulk Acoustic Wave FBAR, SMR technology", Proc. IEEE Ultrasonics Symposium, pp. 1029-1040, 2007.

[18] Comsol Multiphysics, version 3.5, 2009 (www.comsol.com).

[19] A. Tajic, A. Volatier, R. Aigner and M. Solal, "Simulation of solidly mounted BAW resonators using FEM combined with BEM and/or PML", Proc. IEEE Ultrasonics Symposium, pp. 181-184, 2010.

[20]G. G. Fattinger, "BAW resonators design considerations -An overview", Proc. IEEE International Frequency Control Symposium, pp. 762-767, 2008. 



\section{Conclusions and recommendations}

In this final chapter, the general conclusions of this thesis are summarized. In addition, some recommendations for possible future work will be highlighted here. 


\subsection{Conclusions}

This work is an exemplification of the application of optical concepts (wave optics) to acoustic waves in solids. In this thesis, several novel design approaches, derived from optics, have been presented for optimizing dual reflection of longitudinal and shear acoustic waves in the reflector stack of solidly mounted BAW resonators (SMRs). The motivation behind the co-optimization of the acoustical waves in the reflector stack is to minimize acoustic energy losses into the substrate so as to achieve a high acoustical quality factor for the resonators. The design theories were studied and validated using analytical models as well as FEM simulations. Further, we have experimentally proven the concepts by electrical characterization of the resonators.

The design approaches discussed are mainly based on the stop-band theory and principle of diffraction grating. In all the approaches, the velocity ratio of longitudinal to shear wave in the reflector stack layers is important. A more flexible phase error approach is presented that allows the designer to find a trade-off between longitudinal and shear reflection in the stack. The salient feature of all described design approaches is that these can be applied for any material combinations of reflector stacks.

The results obtained from the design approaches have been verified with 2D FEM simulations. A comparison of transmission characteristics from the analytical model and displacement profile from FEM simulations were carried out; the trend in the model and the simulations were found to be in agreement. FEM simulations confirm that the leakage of shear waves into the substrate is suppressed in all shear-optimized stacks from the presented approaches. The approaches have been demonstrated for different material combinations. In all cases, a significantly minimum longitudinal and shear transmission (at resonance frequency) required for high performance resonators were obtained, for various practical reflector material combinations. A performance benchmark of the design approaches has also been presented.

The thickness of the mirror stack affects the dispersion response of the resonator. Therefore, we analyzed the dispersion behavior of the optimized stacks. Depending on the reflector stack design approach, the resonators exhibit dispersion type I or type II. In practice, the resonators should be exhibiting type I in order to use a frame region as the spurious mode suppression scheme for suppressing unwanted ripples in electrical characteristics. A rule of thumb for flipping the dispersion to type I, the preferred dispersion type, is proposed and discussed. 2D FEM simulations demonstrate that the rule of thumb can be applied to both FBARs and SMRs. Although the rule of thumb has been derived under specific assumptions, it provides a sanity guide-line for the BAW designers in deciding the dispersion type of the resonator utilizing the influence of the adjacent layer in the system.

The thesis also presents an experimental investigation of the proposed design approaches to establish the credibility of the approaches. Thanks to NXP semiconductors and the external company, all the design approaches have been 
validated experimentally. Experiments were done for two material combinations: $\mathrm{SiO}_{2} / \mathrm{Ta}_{2} \mathrm{O}_{5}$ and $\mathrm{SiO}_{2} / \mathrm{W}$. The experiments demonstrate that $\mathrm{BAW}$ resonators containing the optimized mirror stacks show a considerable improvement in quality factor compared to devices containing a conventional quarter wave length stack. The results show that design approaches work for the material combinations considered and hence the versatility of the schemes is established. Adaptability of these approaches makes it a potential design guide-line for the devices with minimized acoustic losses into the substrate.

\subsection{Suggestions for further research}

\section{"Known is a drop, unknown is an ocean"- Anonymous}

The work done in this thesis in the context of design approaches for the co-optimization of the reflect stack is nearly comprehensive. However, there is enough room for further research in the related topics described in this thesis.

The design approaches presented in this work had been validated in the description of electrical performance (i.e. the quality factor $Q$ ) of a BAW resonator which is an indirect method for the determination of reflector stack performance. A possible task for the immediate future is therefore to make use of the direct method for the determination of reflector stack performance - the analysis by the laser interformeteric measurement setup. The transmission characteristics of the reflector stack can be directly observed by the vibration amplitude measured by the interferometer both on the surface of the resonator and at the reflector-substrate interface using a glass substrate.

An additional advantage of doing interferometery measurements is that it enables to determine dispersion diagram from the measured vibration fields. The rule demonstrated for flipping the dispersion curves can thus be experimentally verified directly. The rule of thumb extended to FBARs need to be experimentally verified as well. Moreover, the material parameter aspect (for e.g. stiffness or a modified Poisson ratio) which is responsible for the flipping could be a future topic of research for the material science experts.

This thesis aimed at vertical confinement of acoustical energy in the reflector stack in SMRs. Another possible path for acoustic energy leakage is the lateral leakage. The lateral confinement of the acoustical energy is achieved by the use of a frame region, a theoretical optimization of which is still undone. This could open up a new room for research in developing a systematic design procedure for lateral confinement of acoustic waves. While the problem of vertical confinement of energy is a paramount only for SMR type BAW devices, the lateral confinement issue holds for both SMRs and FBARs.

The analysis of the $Q$ factor for predicting the improvement of shear transmission in the reflector stack in SMRs is the approach followed in this thesis. The enhancement in $Q$ factor is further claimed as a consequence of the improvement in shear transmission as the variations of $Q$ values follow the trend of both the longitudinal and shear wave 
transmissions. However, it would be additionally supportive if shear wave transmission can be extracted experimentally. The interferometry technique can only measure out-ofplane motion (vertical displacement) and hence only vibration amplitude of the longitudinal waves. Consequently, even the interferometry techniques fail to measure the amplitude of shear waves directly. Hopefully, more studies will be carried out in this direction to develop a methodology for shear transmission extraction.

This thesis has not investigated other resonator parameters such as coupling coefficient, bandwidth and temperature coefficient of frequency (TCF). The influence of reflector stack design approaches on these parameters need to be studied. The proposal of increasing the top-oxide for flipping of dispersion curves can also affect these parameters.

Multi-band and multi-standard requirements in the current mobile communication system demand tunable BAW. An investigation of tunability of SMRs can be a possible work for future. Tunability can be achieved by several ways. One of it is by using a ferroelectric material (e.g. PZT, Barium Strontium Titanate) as the piezoelectric layer.

The resonators designed in this thesis are for the US-PCS band. BAW devices are also of interest for applications at higher microwave frequencies above $2 \mathrm{GHz}$ for the applications in satellite communications, sensors, radar systems, optical networks and other wireless communications. As frequency increases, BAW devices require thinner films which can be a concern for fabrication. A study in this direction would be appropriate.

Nonlinear behavior in BAW devices is another area which deserves attention of BAW community. No complete theory about the nonlinear effects in BAW resonators has been published so far to our best knowledge. The change in material parameters at high strain levels is a possible cause of nonlinearities in BAW resonators. Nonlinear compact models can be developed to study this. Theoretical models could also be developed with more complicated atomic level description which can be useful for explaining nonlinearities of different material parameters.

BAW resonators being promising candidates for high frequency applications, it is important to analyze the behavior of these structures under harsh environmental and extreme operating conditions. Thermal behavior and failure mechanisms of SMRs at high RF power levels need to be studied. Therefore, as seen in the recent work published in this field, BAW reliability is one of proposed line for future BAW development. 


\section{Appendix A}

\section{COMSOL: Multiphysics modeling and simulation software}

COMSOL Multiphysics (formerly FEMLAB) is a finite element modeling (FEM) simulation software for various physics and engineering applications such as optics, mechanics and heat transport, especially for coupled phenomena or mulitphysics. In addition to conventional physics-based user-interfaces, COMSOL Multiphysics also allows for entering coupled systems of user defined partial differential equations (PDEs) although not used in this thesis. Several application-specific modules are available for COMSOL Multiphysics. For this work, two multiphysics modes were used: plane strain mode and piezo plane strain mode which can be selected either from the MEMS module or the structural mechanics module. Alternatively, it is possible to use only piezo plane strain mode for simulating composite BAW resonators [1], in that case, while entering material properties of the non-piezoelectric layers, decoupled, isotropic option has to be selected from Material model list.

In the performed 2D simulations, $x$ indicates the lateral direction and $y$ indicates the vertical direction. There is a possibility to change the direction of co-ordinate system as well.

The basic equations used in the piezoelectric application mode are the piezoelectric constitutive relations which define how the piezoelectric material's stress $(T)$, strain $(S)$, electric displacement $(\boldsymbol{D})$, and electric field $(\boldsymbol{E})$ interact:

$$
\begin{aligned}
& T=c_{E} S-e E, \\
& D=e S+\varepsilon_{S} E
\end{aligned}
$$

where $e, c_{E}$ and $\varepsilon_{S}$ are the piezoelectric, elastic and dielectric constants respectively.

Applying appropriate boundary conditions is critical for the behavior of the solution. Three mechanical boundary conditions used are fixed, free and symmetric. Simulations 
were done using an axis of symmetry with continuous layers for the reflector and bottom electrode. The discontinuous layers were the top electrode and the piezoelectric layer each with free edge boundaries. At the right hand side, fixed boundary condition was used which assumes the vertical and horizontal displacements to be zero $\left(U_{x}=U_{z}=0\right)$, and at the left hand side, a symmetric boundary condition $\left(U_{x}=0\right.$, $\left.\partial U_{z} / \partial x=0\right)$ were applied. Boundary conditions at the top and bottom were assumed to be free of stress $(\mathrm{T}=0)$.

The electrical boundary conditions used were the following: Potential difference is set to $1 \mathrm{~V}$ between the bottom and top electrodes. Electrical boundary condition defined in comsol depends on the type of analysis. In the frequency response analysis, the electric potential is a.c. Comsol only provides room temperature material properties for modules used. However, temperature dependency could be studied by including the heat transfer module. Alternative option is to account for the temperature variations by appropriately using temperature-dependent material properties in the modules used.

The meshing of the structure is also important for obtaining reliable results especially while performing quantitative analyses. Mapped mesh parameters were used which create rectangular meshes. The minimum element size was set to $1 \mu \mathrm{m}$ in the lateral direction and limited to a size of $0.5 \mu \mathrm{m}$ in the vertical direction. A rule of thumb for adopting an appropriate mesh is to use a mesh size at least equal to $1 / 10^{\text {th }}$ of the wavelength in the structure.

For avoiding the reflections from the substrate, Rayleigh damping condition was applied to the substrate layer given by [2]:

$$
\beta_{\mathrm{dk}}=\frac{1}{Q_{\text {damping }} \cdot 2 \pi \cdot f_{0}}
$$

where $f_{0}$ is the frequency where the structural damping has to be defined. $Q$ damping is the damping constant which was optimized to be 2.5 [2].

Alternatively, perfectly matched layers (PML) can also be used to simulate absorbing boundaries. A PML is strictly speaking not a boundary condition but an additional domain that absorbs the incident radiation without producing reflections.

For the accurate evaluation of $Q$ values, it is advisable to use PML/damping at the outer edge (right hand side) of the resonator. Generation of standing waves due to reflections at the sides of the resonators needs to be avoided as it would provide artifacts related to the mesh and not to the physical object. The $Q$ values calculated in the thesis are after applying the damping given by eq. (A.2).

For comparing the $Q$ values with that obtained from measurements, material damping caused by viscous losses also needs to be included in simulations. In practice, the loss 
should be distributed among all the layers according to the damping constants in different layers. In our simulations, the structural loss factor of $\eta_{\mathrm{cE}}=0.0005$ [3] is accounted in the AlN layer only. This value is entered in the field of isotropic loss in the structural damping list. Another possibility is to include material damping for the lightest metal in the stack (say Aluminum, a $Q_{\mathrm{m}}$ value of 50 [2]).

For analyzing the impedance and dispersion curves, frequency response and eigen frequency analyses were used respectively. The impedance curve is computed from Comsol frequency response analysis by post processing, which is obtained by charge integrated on the top electrode boundary. Eigen frequencies calculated by the Comsol are related to the eigen values calculated for the mechanical structure returned by the solver.

\section{Impedance calculation by Comsol post processing:}

The results available from the frequency response analysis are the mechanical displacement and the electric displacement. However, the goal is to compute the electrical impedance, $\mathrm{Z}$, which is the ratio of applied potential (V) to the total current $\left(\mathrm{I}=j \omega Q_{e}\right)$ given by:

$$
Z=\frac{V}{j \omega Q_{e}}
$$

where $Q_{\mathrm{e}}$ is the total electric charge and $\omega$ the angular frequency.

The total electric charge can be calculated by considering the BAW resonator as a parallel-plate capacitor. The integral of electric displacement in the thickness direction in the area under the top electrode results in the total electric charge. Hence $Q_{\mathrm{e}}$ is given by:

$$
Q_{e}=\iint_{\mathrm{A}} 2 . D \mathrm{y} \_ \text {smppn.L, }
$$

where Dy_smppn is the displacement in the vertical direction in the piezoplane strain mode in comsol and $\mathrm{L}$ is the third dimension. The 2 in the equation is because of the half structure simulation.

[1] http://www.comsol.com/showroom/gallery/5784/

[2] Private communication with J. Ruigrok, NXP semicondutors, NL.

[3] Private communication with the external company. 



\section{Appendix B}

\section{FEM simulation vs. Mason model}

For a verification of FEM simulation results in Comsol, the Mason transmission line model discussed in section 2.2.1 was implemented in Mathcad for samples 1, 2, 3 and 7 discussed in Table 5.1. 2D FEM simulations were performed imitating 1D situation by using continuous layers with symmetric boundary conditions at the left and right edges. The boundary conditions at bottom and top were assumed to be free. Figure B.1 shows the comparison of results from the FEM simulation and the analytical model, showing good agreement for various (anti)resonance frequencies.

Velocities of the longitudinal $\left(v_{\mathrm{L}}\right)$ and shear waves $\left(v_{\mathrm{s}}\right)$ and the mass density $(\rho)$ of the material are the input material parameters in the Mason model. In Comsol simulations, Young's modulus (E), Poisson's ratio $(\sigma)$ and mass density are the material parameters. These parameters are inter-related by the following equations:

$$
E=\frac{\left(c_{11}-c_{12}\right) \cdot\left(c_{11}+2 c_{12}\right)}{\left(c_{11}+c_{12}\right)}
$$

where $c_{11}$ and $c_{12}$ are elastic material constants or stiffness coefficients in the stiffness matrix and are interconnected by:

$$
c_{12}=c_{11}-2 c_{44}
$$

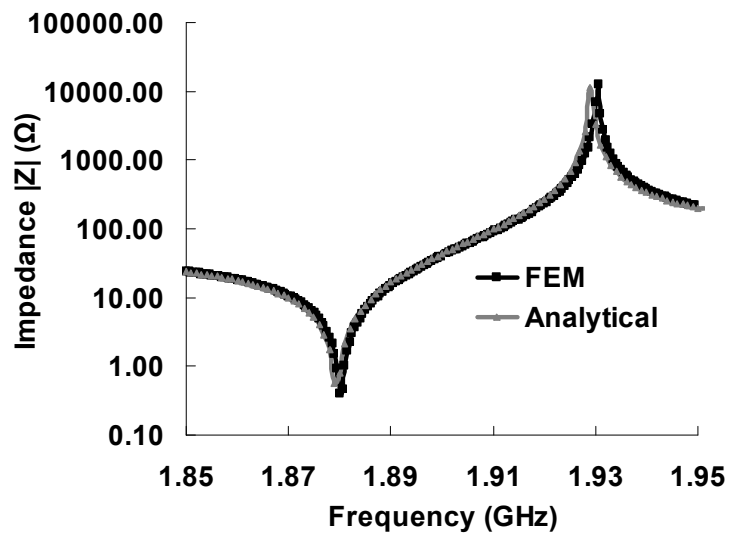

(a)

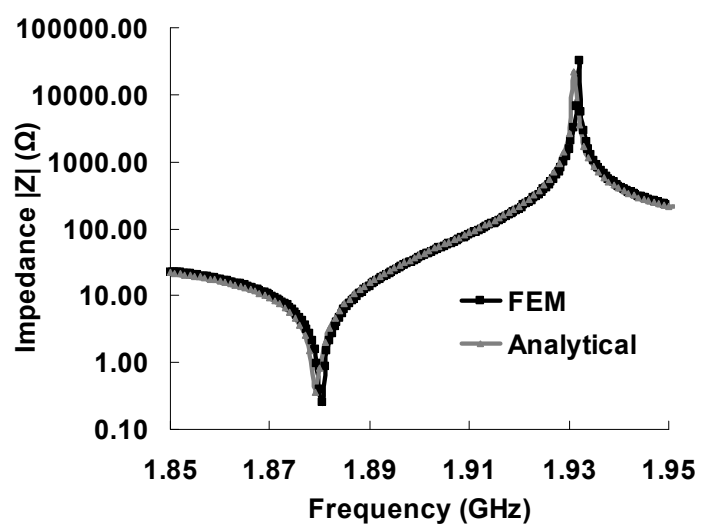

(b)

Figure B.1 : $\quad 2 \mathrm{D} \mathrm{FEM} \mathrm{simulated} \mathrm{and} \mathrm{the} 1 \mathrm{D}$ analytical impedance vs. frequency of the resonator with $\mathrm{SiO}_{2} / \mathrm{Ta}_{2} \mathrm{O}_{5}$ reflector stacks (a) sample 1 and (b) sample 2 (refer to Table 5.1). 2D FEM simulations were performed by imitating the situation of the device in $1 D$. The $1 D$ analytical impedance was calculated using Mason's transmission line model. Active device area is $140 \times 140 \mathrm{\mu m}^{2}$. 


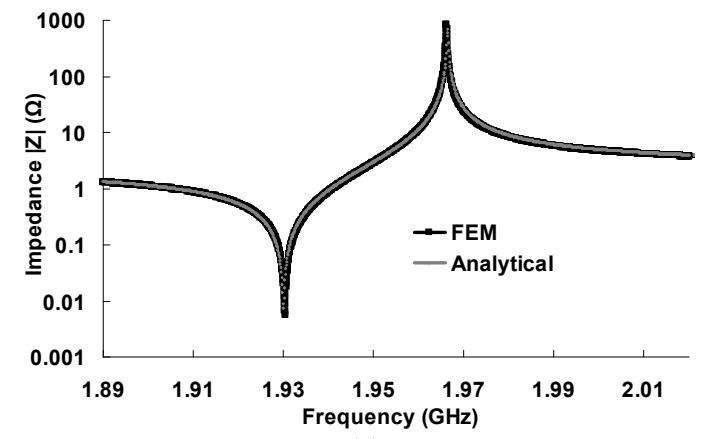

(a)

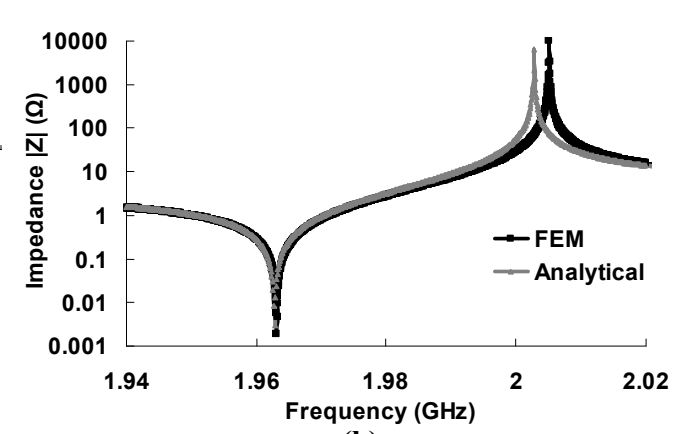

(b)

Figure B.2 : $\quad 2 D$ FEM simulated and the $1 D$ analytical impedance vs. frequency of the resonator with $\mathrm{SiO}_{2} / \mathrm{W}$ reflector stacks (a) sample 3 and (b) sample 7 (refer to Table 5.1). 2D FEM simulations were performed by imitating the situation of the device in $1 D$. The $1 D$ analytical impedance was calculated using Mason's transmission line model. Active device area is $140 \times 140 \mathrm{\mu m}^{2}$.

The stiffness coefficients $c_{44}$ and $c_{11}$ are related to $v_{\mathrm{L}}$ and $v_{\mathrm{s}}$ as:

$$
\begin{aligned}
& v_{\mathrm{L}}=\sqrt{\frac{c_{11}}{\rho}}, \\
& v_{\mathrm{S}}=\sqrt{\frac{c_{44}}{\rho}} .
\end{aligned}
$$

The Poisson ratio can be calculated for $v_{\mathrm{L}}$ and $v_{\mathrm{s}}$ by:

$$
\sigma=\frac{1-2\left(\frac{v_{\mathrm{S}}}{v_{\mathrm{L}}}\right)^{2}}{2-2\left(\frac{v_{\mathrm{S}}}{v_{\mathrm{L}}}\right)^{2}}
$$

In the Mason model, isotropic material parameters were used all layers except the piezoelectric layer. However, the hexagonal symmetry of Aluminium Nitride was not accounted in the Mason model. To drive the Mason model correctly, it has to be ensured that $v_{\mathrm{L}}$ provided is the piezoelectrically stiffened velocity given by:

$$
v_{\mathrm{L}}=\sqrt{\frac{c_{E}+e^{2} / \varepsilon_{S}}{\rho}},
$$

where $e, c_{E}$ and $\varepsilon_{S}$ are the piezoelectric, elastic and dielectric constants in the thickness direction respectively. 


\section{Appendix C}

\section{$Q$ extraction method}

The scaling of the $Q$ factor with resonator dimensions provides important information about the nature of the loss mechanisms involved. A simple relation for the $Q$ factor can be formulated by dividing the total loss into a contribution scaling with the area (1D loss through the reflector, material loss) and a contribution scaling with the perimeter (e.g. acoustic radiation outwards). $Q$ is proportional to the ratio of the stored energy to lost energy. The stored energy $\left(E_{\mathrm{s}}\right)$ scales with the area of the resonator. The lost energy is the sum of energy lost through the reflector $\left(L_{\mathrm{r}}\right)$ and the energy lost through the edge $\left(L_{\mathrm{e}}\right)$. $L_{\mathrm{r}}$ scales with the area $(A)$ and $L_{\mathrm{e}}$ scales with the perimeter $(P)$ of the resonator. Hence $1 / Q$ can be defined as:

$\frac{1}{Q} \equiv \frac{\text { Lost Energy }}{\text { Stored Energy }}=\frac{\left(L_{\mathrm{r}}+L_{\mathrm{e}}\right)}{E_{\mathrm{s}}}=\frac{L_{\mathrm{r}}}{E_{\mathrm{s}}}+\frac{L_{\mathrm{e}}}{E_{\mathrm{s}}}$.

In eq. (C.2), in the first term, both the numerator and the denominator scale with the area, so the whole term is area-independent. The second term scales with perimeter/area $(P / A)$. Hence, the following holds:

$$
\frac{1}{Q_{\mathrm{A}}}=\mathrm{A}_{1}+\mathrm{A}_{2} \cdot \frac{P}{A},
$$

where $\mathrm{A}_{1}$ and $\mathrm{A}_{2}$ are constants.

For resonators without a frame region, $Q_{\mathrm{A}}$ strongly depends on the $P / A$ ratio, indicating that the loss of these resonators is dominated by edge effects. By applying a frame region, the dependence on $P / A$ nearly vanishes and the loss is then dominated by area effects. The vertical axis cutoff gives the value of $Q_{\mathrm{A}}$ which is dominated by area effects and hence $1 \mathrm{D} Q$. A low extrapolated value of the vertical axis cutoff shows that the loss contribution scaling with area is relatively small.

The $\mathrm{P} / \mathrm{A}$ scaling method for $\mathrm{Q}$ extraction is verified in 2D FEM simulations. Since 2D simulations were performed, the perimeter/area translated to 2/width in 2D. Figure C.1 is a plot showing the scaling of quality factor at anti-resonance $Q_{A}$ for a series of resonators with different widths with the phase error optimized stack (sample 5, Table 5.1) simulated in $2 \mathrm{D}$ FEM. The $Q_{\mathrm{A}}$ values are calculated using the bandwidth method (eq. 2.17) discussed in chapter 2. 


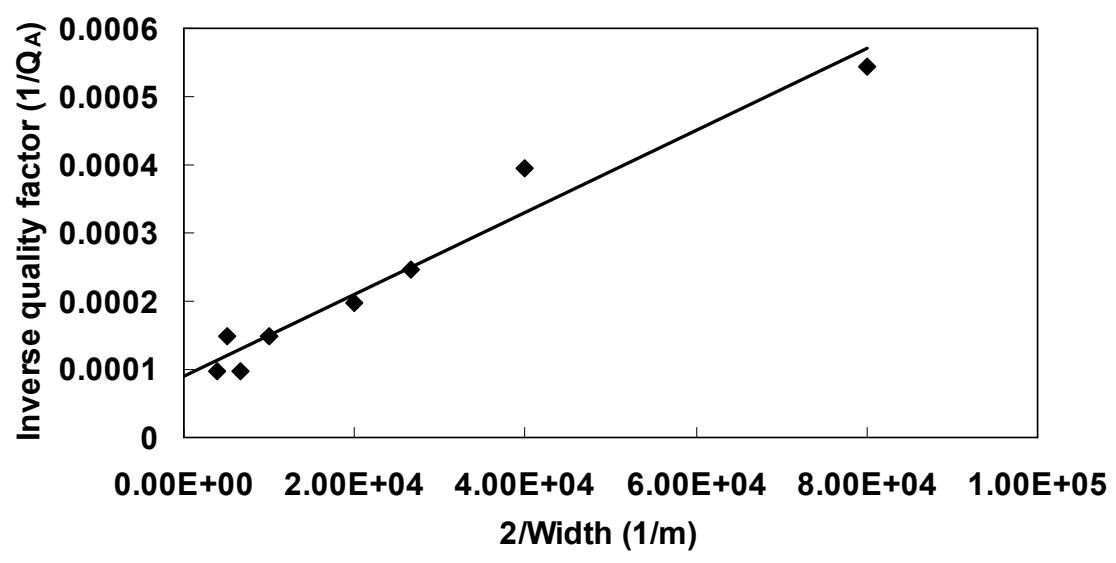

Figure C.1 : $\quad 2 D$ FEM simulated data showing scaling of the quality factor at anti- resonance, $Q_{\mathrm{A}}$, for resonators with various widths with $\mathrm{SiO}_{2} / W$ phase error optimized stack. The extrapolated value at the vertical axis gives us the $1 D Q_{A}$.

The vertical axis cutoff gives the value of $1 \mathrm{D} Q_{\mathrm{A}}$. From the plot an extrapolated value of 0.000098 is obtained which gives a $1 \mathrm{D} Q_{\mathrm{A}}$ value of 10,204 . This is compared with the $Q_{\mathrm{A}}$ value obtained from 1D Mason model which gave a value of 9306 . We also did a comparison of $Q_{\mathrm{A}}$ value obtained from FEM simulation for a 1D configuration boundary conditions with continuous layers. This gave a $Q_{\mathrm{A}}$ value of 10,144. The same frequency step-size was maintained in all these simulations. This exercise supports that the extrapolated $Q_{\mathrm{A}}$ value at the vertical axis cut-off is indeed the $1 \mathrm{D} Q_{\mathrm{A}}$. 


\section{Summary}

Thin-film bulk-acoustic-wave (BAW) devices are used for RF selectivity in mobile communication system and other wireless applications. Currently, the conventional RF filters are getting replaced by BAW filters in all major cell phone standards. In this thesis, we study solidly mounted BAW resonators (SMR) which are the building blocks of these filters. The good selectivity offered by the BAW resonators makes them excellent components for inter-stage filters and duplexers for mobile applications.

Modelling and development of high performance thin-film BAW devices is a topic of research gaining attention from BAW specialists around the world. The quality factor of an SMR is limited by acoustic substrate losses, because the reflector stack is traditionally optimized to reflect longitudinal waves only. This thesis presents several novel design approaches for optimizing the reflector stack for dual reflection of longitudinal and shear acoustic waves in view of achieving high acoustical quality factor.

Two main concepts are studied in this thesis. The first one is the optimization of the reflector stack and the second one is the influence of reflector stack design on the acoustic dispersion of the resonators. The realized devices utilizing the concepts are experimental validated.

Two main reflector stack design approaches - the stop-band theory and the diffraction grating based approaches - derived from the optics background were presented. The approaches were successfully verified with 1D-model and 2D FEM simulations. The approaches had been demonstrated for different material combinations and in all the cases a minimum transmission of $-25 \mathrm{~dB}$ and $-20 \mathrm{~dB}$ at resonance frequencies were obtained for longitudinal and shear waves respectively, for various practical reflector material combinations. The standard quarter wave stack shows similar transmission for longitudinal waves, but very high transmission (e.g. $-0.59 \mathrm{~dB})$ for shear waves. Hence the optimized reflector stacks show an efficient reflection of both longitudinal and shear waves.

The reflector stack design affects the acoustic dispersion of the resonators. The resonators adopting reflector stack designs with stop-band theory based approaches resulted in type II dispersion. On the positive side, the ones adopting the diffraction grating based stacks exhibited type I dispersion which is the preferred dispersion type 
for frame region functioning. We derived a rule of thumb for flipping the dispersion relation of SMRs to type I, by assuming the shear confinement in the top-oxide layer of the reflector stack. The rule was also extended for free standing bulk acoustic wave resonators (FBARs).

The experimental verification of the design approaches was presented. The extracted 1-D acoustical quality factor for the realized shear optimized devices with a stop-band theory based $\mathrm{SiO}_{2} / \mathrm{Ta}_{2} \mathrm{O}_{5}$ reflector stack was increased to around 3300 . The versatility as well as credibility of the approaches was verified by another set of experiments on $\mathrm{SiO}_{2} / \mathrm{W}$ stacks. The experiments established the theoretical prediction of the optimum reflection when the optimization parameter is between 1 and 2 . It was found that the quarter-wave like stacks with an increased top-oxide layer thickness, showed a higher extracted 1D quality factor. With DGM stacks the highest experimental quality factor was obtained. FEM simulations were performed for further understanding of the experimental results and the extracted values were corroborated with the trend observed in the simulations. 


\section{Samenvatting}

Bulk-Acoustic-Wave (BAW) filters, gemaakt met dunne-film technologie, worden gebruikt om RF signalen te selecteren in mobiele communicatie en andere draadloze toepassingen. Dit soort filters is vernoemd naar de akoestische golf die door de bulk van het materiaal doorgegeven wordt.

Conventionele RF filters worden steeds vaker vervangen door zulke BAW filters in de belangrijkste standaarden voor (mobiele) communicatie. In dit proefschrift onderzoeken we BAW resonatoren die vast op een substraat vervaardigd worden (zogeheten SMR componenten). Deze BAW resonatoren bieden een hoge selectiviteit, waardoor het uitstekende componenten zijn om zogeheten inter-stage filters en duplexers mee te vervaardigen voor mobiele communicatie. De selectiviteit wordt gekenmerkt door de zogeheten kwaliteitsfactor ( $Q$-factor); een hoge $Q$-factor betekent dat slechts een zeer klein deel van het spectrum wordt doorgelaten.

Het modelleren en ontwikkelen van BAW resonatoren met een hoge kwaliteitsfactor krijgt steeds meer aandacht van BAW specialisten overal ter wereld. De kwaliteitsfactor van een SMR wordt beperkt door het verlies van akoestische golven door het substraat. Met een reflectorpakket tussen de BAW resonator en het substraat wordt dit tegengegaan; echter nog niet al te effectief, doordat het pakket veelal geoptimaliseerd is voor het reflecteren van longitudinale golven. In dit proefschrift worden verschillende nieuwe ontwerpen gepresenteerd met een hogere kwaliteitsfactor. Dit wordt bereikt door het reflectorpakket te optimaliseren voor de reflectie van zowel longitudinale als transversale akoestische golven. Naast optimalisatie van de reflectie is ook de invloed van de reflector op de dispersie in de resonator een belangrijk onderdeel van het gepresenteerde onderzoek. In dit proefschrift worden systemen gepresenteerd waarbij twee manieren voor optimalisatie zijn toegepast; de goede werking van de systemen is experimenteel aangetoond.

We gebruiken twee verschillende manieren om het reflectorpakket te optimaliseren; één gebaseerd op de 'stop-band' theorie en een andere gebaseerd op het gebruik van een diffractie-raster. Beide methoden zijn ontleend aan de optica. De twee ontwerpen volgend uit deze optimalisaties zijn gemodelleerd, in één dimensie en in twee dimensies, met behulp van de eindige-elementen methode. Beide ontwerpen zijn ook experimenteel getest met verschillende materiaalcombinaties voor het reflectorpakket. Bij de 
resonantiefrequentie is de minimale transmissie $-25 \mathrm{~dB}$ voor longitudinale golven en $-20 \mathrm{~dB}$ voor transversale golven. Een traditionele kwart-golflengte reflector geeft een vergelijkbare transmissie voor longitudinale golven maar een zeer hoge transmissie (b.v. $-0.5 \mathrm{~dB}$ ) voor transversale golven. Met de geoptimaliseerde reflectorpakketten is een goede reflectie van beide soorten golven mogelijk.

Het ontwerp van het reflectorpakket heeft een grote invloed op de dispersie in de resonator. De resonatoren met een reflector van het stop-band type hebben een zogeheten type II dispersie. De systemen met een diffractie-raster reflector hebben een dispersie van het meer gewenste type I.

We presenteren een vuistregel voor het ontwerp van SMR systemen waarmee een type I dispersiepatroon verkregen kan worden. Deze regel is gebaseerd op het concentreren van alle transversale golven in de bovenste oxidelaag van het reflector pakket. Deze methode kan ook gebruikt worden voor vrijstaande, niet vast op een substraat gemaakte resonatoren (zogeheten FBAR systemen).

De verschillende ontwerpen zijn experimenteel gevalideerd, waarvan de resultaten in dit proefschrift zijn opgenomen. De één-dimensionale kwaliteitsfactor is 3300 voor systemen met een op de stop-band theorie gebaseerd reflectorpakket van $\mathrm{SiO}_{2}$ en $\mathrm{Ta}_{2} \mathrm{O}_{5}$. De toepasbaarheid en de geloofwaardigheid van de gebruikte ontwerpmethodes is uitgebreid door een studie naar het gebruik van andere materialen, te weten $\mathrm{SiO}_{2}$ en $\mathrm{W}$. Theoretische voorspellingen over de optimale reflectie zijn geverifieerd door middel van een zogeheten optimalisatieparameter $c$ tussen 1 en 2. De kwart-golflengte reflectorpakketten met een dikkere bovenste oxidelaag hebben een hogere één-dimensionale kwaliteitsfactor. De reflectoren van het diffractie-raster type geven de hoogste kwaliteitsfactor. Numerieke simulaties zijn uitgevoerd teneinde een beter begrip te krijgen van de experimentele resultaten; de gemeten waarden komen overeen met het gedrag dat door de simulaties is voorspeld. 


\section{Acknowledgements}

Acknowledgements are never complete; the list of the people who are thanked can be incomplete as the thoughts that are acknowledged.

Dear reader, thanks for your interest in this work. In these last pages, you will find my sincere thanks to many people who filled my life as a PhD student with some best moments.

First of all, I would like to thank Jurriaan Schmitz for providing me with the opportunity to carry out my research as a graduate student at the University of Twente (UT), Enschede. Jurriaan, thanks for being my promoter, extending your kind support and advices. You have always been encouraging with my research.

I express my sincere gratitude to my supervisor Ray Hueting, who always has time for his students. In these four years he always had time for me. I could always walk in to his room for discussions. Ray, apart from learning many things, it was fun working with you. Thanks for introducing me to BAW and for giving me the freedom in my research. Thank you for your valuable suggestions and contributions towards this work. You have always motivated me to think aloud. Special thanks to your quick comments on the thesis which also helped me finish it in time.

My heartfelt thanks to my supervisor at NXP Semiconductors, André Jansman to whom I owe for his consistent interest in the BAW project even after NXP's closure of the division. André, I really enjoyed the time being at NXP, learning along with you various aspects of BAW physics. For me, you are more a story teller than a supervisor, a mentor who inculcated BAW physics into my budding mind making the toughest subject of acoustics as easy as listening to a story. The short notes I jotted down at NXP, later on became a BAW manual for me. Your involvement in the project has greatly contributed to the success of this work.

I use this opportunity to thank Jaap Ruigrok, NXP Semiconductors, for his enthusiastic support for Comsol simulations. Jaap, thanks for teaching me Comsol, warning me with the limitations of the FEM simulations and helping me to interpret the colorful pictures in terms of device performance. You have helped me with many critical steps in BAW 
resonator simulations. Back here in the university, I am thankful for your fast responses via emails, whenever I had a query.

I would like to thank the former BAW team of NXP Semiconductors specially Remco Strijbos, Henk Bosch and Jan-Willem Loebeek for the fabricated devices. Thanks also to the BAW team of an external company for the fabricated devices and the measurement data. Special thanks to the concerned person at the external company for coming up with the proposal of collaboration. Thanks for the telephonic discussions that aided the successful fabrication of the stacks. Many thanks for the prompt actions which helped me obtain the intended results in time.

I thank René de Ridder, IOMS, for stimulating discussions on the diffraction grating approach. Thanks also to Henk de Vries, ICD, for helping me with the RF measurements. I also wish to acknowledge the financial support by the Dutch Ministry of Economic Affairs in the framework of the Point one project MEMSLand. Thanks to the PhD promotion committee members for taking time to read my thesis and for being part of my defense.

Work apart, PhD at UT was also fun with the SC group. The first person who comes to my mind when I think of our group is Annemiek; she is the boss of SC. Annemiek, thanks for the ever willingness to help me and your kind support through out my PhD. I would like to thank all my SC colleagues who made my stay in the group memorable. First in the list are my AIO roommates: Balaji, Boni, Buket, Giulia, Hao, Naveed, Tom and Vidhu; you are the best colleagues ever. I enjoyed so much with you that it was difficult for me to shift to the writing room. Thanks for supporting me especially when I was writing the thesis. I would like to extend my thanks to my other colleagues: Alexey, Cora, Rob, Sander, Tom Aarnink, Jiahui and Pietro. Thanks also to my ex-colleagues: Joost, Victor, Bijoy, Arjen, Jiwu, Deepu, Ihor, Erik, Jan-Laurens and Alfons. Bijoy, thanks for all the help. Special thanks to Joost for the dutch translation of the summary.

Thanks to the other floor mates especially Cheng Wei (for the good morning wishes on Sunday) and Maurizio for the occasional chit-chats. I would like to thank our NXP girls' team: Ruoxuan, Jing and Noémie for the ultimate fun we had in Eindhoven. Thanks to the other friends I met in the campus: Rani, Tamalika, Jincy, Arpita, Nupur, Hrudya and Saleha. Lily (ammachy), it was my pleasure to get to know you. Thanks for the wonderful moments we spent together, for the Sunday coffees and for being my dutch oma here. Padmakumar-Gayathri, Mercy-Shaji, Imran-Sameena, I always felt at home whenever I visited you. Sameena bhabhi, hats off to you for your exquisite cuisines, I really enjoyed it. In addition, I should not forget to mention the friends I met in Eindhoven: Denny-Abraham, Jinesh-Devi and Biju chettan.

I am thankful to Buket and Joost for being my paranymphs, ensuring your support and sharing my happiness.

I thank my previous project supervisors in India: M. Umapathy, G. Uma, S. Raghavan, K. J. Vinoy and C. K. Aanandan for motivating me towards academic achievements. 
I would also like to thank my family and the intimate ones for their support. Banu (Mubassira) thanks for being with me for all these years. Sr. Immaculata, thanks for your prayers and support especially when I was writing the thesis.

I would like to express my most sincere gratitude to my parents (Jose Marangattu \& Ruby Jose), without their consistent encouragement and loving support I would not have come so far. Thanks for your prayers and support in all my decisions. Thanks to Smitha, Martin Jose, Sonu and my cute little nieces- Helen and Rose for their love and support. Thanks to Baby uncle and Suji chettan for their support and advices on my career. Above all, I thank God almighty for all the countless blessings and guiding me towards the cheerfulness of life.

I take a word to thank anyone that I might have forgotten in this list.

Thank you all.

Enschede, November $2011 \quad$ Sumy Jose 



\section{About the author}

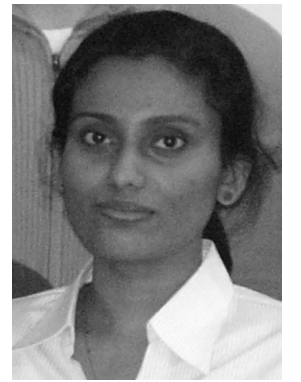

Sumy Jose was born in Kerala, India in 1982. She received her bachelor degree from MG University (Kerala) in Physics in 2002. She graduated in Applied Electronics from National Institute of Technology (NIT), Tiruchirappally, India in 2004. Her Master thesis on "Design and Simulation of PVDF-MOSFET based MEMS Acoustic Sensor" was done at Naval Physical and Oceanographic Laboratory (NPOL), Cochin, India. She joined as a project assistant at the Indian Institute of Science (IISc), Bangalore in 2005 where she has been involved in the research projects in the area of RF MEMS design. In December 2007, she joined the Semiconductor Components group at the University of Twente, The Netherlands, as a PhD student to work on BAW resonators. The results of that work are presented in this thesis. During her PhD, she had consistent contributions towards IEEE International Ultrasonics Symposium (IUS) 2009, 2010 and 2011. Her primary research interests include physics based multi-domain modelling and design of acoustic and MEMS devices. 
